\title{
Frequency Domain Tests for Assessing Dependency Characteristics of Stationary Time Series via Tapering
}

$$
\text { by }
$$

\section{Gray Barski}

A thesis submitted to the Faculty of Graduate Studies and Postdoctoral Affairs in partial fulfillment of the requirements for the degree of

\author{
Master of Science \\ in \\ Statistics \\ Carleton University \\ Ottawa, Ontario, Canada
}

(C) 2019

Gray Barski 


\begin{abstract}
We present a handful of periodogram-based test statistics underneath two statistical procedures that assess the characteristics of a stationary time series in a manner that is similar to the Gromykov et al.(2018) and Ould Haye \& Phillippe (2019). We incorporate tapering into these statistics by utilizing a cosine bell taper and a new class of tapers which we term as "random tapers". In the first procedure we test for short memory versus long memory. In the second we test for dependence against the presence of a trend. We demonstrate that under the null, both procedures' limiting distributions are easily obtainable and follow Gamma-like distributions. Moreover, we evaluate the P-value and Empirical Power plots accrued by each test and find that they yield precise results of empirical size. The tests are also implemented into two sets of realworld data and the accrued results are presented.
\end{abstract}




\section{Contents}

1 Introduction $\quad 6$

1.1 Motivation .......................... 7

1.2 Outline . . . . . . . . . . . . . . . . . . 13

2 Theoretical Review $\quad 14$

2.1 Time Series Processes . . . . . . . . . . . . . . . . . . . 14

2.2 Time Domain . . . . . . . . . . . . . . . . . . . . 15

2.3 Frequency Domain . . . . . . . . . . . . . . . . . . . . . . 19

2.4 Two Approaches to Assessing the Characteristics of a Time Series . . 21

2.5 Periodogram . . . . . . . . . . . . . . . . . 25

2.6 Tapering . . . . . . . . . . . . . . . . . 27

3 Literature Review 30

3.1 Log-Periodogram Regression . . . . . . . . . . . . . . . . . . . 30

3.2 Distribution of Periodogram Ordinates . . . . . . . . . . . . . 31

3.3 Ramifications of Implementing the Cosine Bell Taper . . . . . . . . . 33

3.4 Time Domain Tests for Short v. Long Memory . . . . . . . . . . . 38

$3.4 .1 \mathrm{~V} / \mathrm{S}$ Statistic . . . . . . . . . . . . . . . 41

3.5 Frequency Domain Tests for Short v. Long Memory . . . . . . . . . . 44

3.5.1 LR Statistic . . . . . . . . . . . . . . . . . 44

3.5.2 $\mathrm{Q}_{n, m}(\mathrm{~s})$ Statistic ......................... 44 
3.6 Testing for Dependence v. Non-Stationarity . . . . . . . . . . . . 48

3.6.1 $\mathrm{T}_{n}(\mathrm{~d})$ Statistic . . . . . . . . . . . . . . . 48

3.6.2 $\mathrm{Q}_{n, m}(\mathrm{~s}, \mathrm{~d})$ Statistic . . . . . . . . . . . . . 49

4 Methodology $\quad 52$

4.1 Cosine Bell Taper . . . . . . . . . . . . . . . . . . . . . 53

4.1.1 Asymptotic Distribution Within the First Procedure . . . . . 63

4.1.2 Asymptotic Distribution Within the Second Procedure . . . . 66

4.2 Random Tapering . . . . . . . . . . . . . . . . . . . . . . . 68

4.2.1 Asymptotic Distribution Within the First Procedure . . . . . 74

4.3 Testing Procedure Methodology . . . . . . . . . . . . . . 76

4.3.1 First Procedure . . . . . . . . . . . . . . . . . . . . . . 78

4.3.2 Second Procedure . . . . . . . . . . . . . . . . 79

4.4 Limiting Distribution . . . . . . . . . . . . . . . . . . . 81

5 First Procedure Findings $\quad 83$

5.1 Null Hypothesis . . . . . . . . . . . . . . . . . . . . . . . . . . . . . . 83

$5.1 .1 \mathrm{Q}_{n, m}^{T}(s) \ldots \ldots \ldots \ldots \ldots \ldots$

$5.1 .2 Q_{n, m}^{R T}(s) \ldots \ldots \ldots \ldots \ldots \ldots$

5.2 Alternative Hypothesis . . . . . . . . . . . . . . . . . . 88

5.3 Comparision . . . . . . . . . . . . . . . . . 91

5.3.1 Null Hypothesis . . . . . . . . . . . . . . . . . . . . . 91 
5.3.2 Alternative Hypothesis . . . . . . . . . . . . . . . . . 93

5.4 Conclusion . . . . . . . . . . . . . . . . . . . . . . 95

6 Second Procedure Testing Results 96

6.1 Null Hypothesis . . . . . . . . . . . . . . . . . . . . . . . . . 96

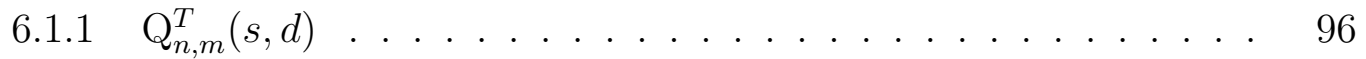

$6.1 .2 \mathrm{Q}_{n, m}^{T^{\prime}}\left(s, d_{G P H}^{T}\right) \ldots \ldots \ldots \ldots \ldots \ldots \ldots \ldots \ldots \ldots \ldots \ldots \ldots \ldots$

6.2 Alternative Hypothesis . . . . . . . . . . . . . . . . . . . . . 99

6.3 Comparison . . . . . . . . . . . . . . . . . . . . . 101

6.4 Conclusion . . . . . . . . . . . . . . . . . . . . . . . . . . 101

7 Findings - Real World Example 102

7.1 First Procedure - SP500 _ . . . . . . . . . . . . . . . 102

7.2 Second Procedure - VIX . . . . . . . . . . . . . . . 105

$\begin{array}{lll}8 & \text { Conclusion } & 109\end{array}$

9 Appendix A 112

9.1 Additional Concepts . . . . . . . . . . . . . . . . . . . . . . . . . 112

9.2 Theoretical Content . . . . . . . . . . . . . . . . . . . . . . 119

10 Appendix B 162

10.1 Numerical Code . . . . . . . . . . . . . . . . . . . . . . . . 162

11 References 181 
Table 1: Notation and Conventions

$$
\begin{aligned}
& \stackrel{\mathbb{D}}{=}:=\text { Equal in Distribution } \\
& \stackrel{\mathbb{P}}{\longrightarrow}:=\text { Converges in Probability. } \\
& \stackrel{\mathbb{D}}{\longrightarrow}:=\text { Converges in Distribution. } \\
& \exp (c):=\text { Exponential Distribution with Mean }=1 / c . \\
& U(a, b):=\text { Uniform Distribution Defined on }[a, b] \text {, where } a<b \text {. } \\
& N_{s}\left(\mu_{s}, \Sigma_{2 s, 2 s}\right):=\text { Multivariate Normal Distribution with Mean Vector } \mu_{s} \text { and } \\
& \text { Covariance Matrix } \Sigma_{s, s} \text {. } \\
& \Gamma(s, k):=\text { Gamma Distribution with Parameters s and k. } \\
& \stackrel{i . i . d}{\sim}:=\text { Independent and Identically Distributed. } \\
& \bar{g}:=\text { Complex Conjugate of the Function } g \text {. } \\
& \sim:=\text { Asymptotically Equivalent. } \\
& L_{p}:=\text { Class of p-Integratable Functions Defined on } \mathbb{R} \text {. } \\
& X_{n}=O_{p}(1):=\text { The Sequence of Random Variables } X_{n} \text { is Bounded in Prob- } \\
& \text { ability, That is if for Every } \epsilon>0 \text { There Exists } \delta>0 \text {, Such } \\
& \text { That } P\left(\left|X_{n}\right|>\delta\right)<\epsilon, \forall n \text {. } \\
& X_{n}=o_{p}(1):=\text { The Sequence of Random Variables } X_{n} \text { Converges in Proba- } \\
& \text { bility to Zero, that is } X_{n} \stackrel{\mathbb{P}}{\longrightarrow} 0 \text {. } \\
& a_{n}=O\left(b_{n}\right):=\text { The Sequence } a_{n} \text { is Bounded by } b_{n} \text {, That is if and only if } \\
& \text { There Exists } C>0, N>0 \text { Such That }\left|a_{n}\right| \leq C b_{n} \text {, if } n>N \text {. } \\
& a_{n}=o\left(b_{n}\right):=\text { The Sequence } a_{n} / b_{n} \rightarrow 0 \text {, as } n \rightarrow \infty \text {. }
\end{aligned}
$$




\section{Acknowledgements}

I would like to thank my supervisor Dr. Mohamedhou Ould Haye for the time, effort, and resources he gave to me over the course of this thesis. Furthermore, I express specific gratitude towards Drs. Raluca Balan and Natalia Stepanova for participating on my graduate committee and for the valuable advice they provided in regards to the content of the thesis.

I would also like to thank my friend and fellow classmate, Mohammad-Amin Nabavi for the support and motivation he provided me with. I specifically appreciate the wisdom and encouragement he gave me. Finally, I would like to thank my parents, grandparents, and community at The Met for their prayers and support.

\section{Introduction}

When evaluating a time series there are core characteristics that a researcher is primarily concerned with assessing such as stationarity. Within a certain class of stationary time series, further classifications of a processes second-order characteristics can be made by analyzing the correlation structure as Giraistis et al.(2012, Page 33) state. The concepts of negative, short, and long memory are examples of these as they allow for a processes' dependence to be further described. The dependence and stationarity a series possesses has implications on the asymptotic properties of conventional estimators of the mean, variance, and autocorrelation of the process. There 
are existing procedures which assess these second-order characteristics which have been developed within the time domain. However, recently there has been research endeavours that allude to the potential of developing robust statistical methods underneath the frequency domain by utilizing the periodogram in some capacity. We build upon these procedures and present tests that attempt to distinguish between the second-order characteristics of a stationary linear process. The first procedure tests for short memory against the alternative of long memory. The second procedure evaluates negative, short, or long memory under the null versus the presence of a deterministic or stochastic trend within the alternative. We now proceed to present the motivation for this research.

\subsection{Motivation}

Given that the second-order characteristics of a time series have ramifications on the asymptotic properties of conventionally implemented estimators, it should be of no surprise that methods used to distinguish between these characteristics are desired. The procedures that appear to be the most commonly used for testing for short memory against the alternative of long memory are developed under the time domain. Examples of these are Lo (1991) and Giraistis et al.(2003). However, these time domain procedures often involve asymptotic distributions that are difficult to obtain as some of these tests yield a limiting distribution under the null hypothesis that requires the use of stochastic integration. Moreover, another characteristic of these 
time domain tests is that they are known to suffer from high empirical size. Under the frequency domain Lobato \& Robinson (1998) developed a procedure for assessing between short and long memory. This test possessed a simple limiting distribution while obtaining precise empirical size. These two features acted as improvements on the drawbacks of the time domain tests. However, similar to the procedures from the time domain, the test generated results that indicate a difficulty when calibrating the test's window parameters. As Gromykov et al.(2018) state this was observed through the substantial loss in empirical power when attempting to obtain a precise value of empirical size. Moreover, the authors in Gromykov et al.(2018) presented a new statistical test underneath the frequency domain that assesses for short memory versus the alternative of long memory. This test yielded notable results such as a simple limiting distribution under the null, precise empirical size values, and stable window parameters. As one will note, each of these characteristics act as improvements on the undesirable aspects of the previously discussed time domain tests.

Another statistical procedure that uses a compilation of periodogram ordinates in a similar manner to Gromykov et al.(2018) has been proposed in the upcoming paper of Ould Haye \& Phillippe (2019). This procedure tests for dependence against the alternative of non-stationarity and therefore appears to possess greater applicability in real world applications as there is a difficulty in distinguishing between long memory and non-stationarity in practice. For instance, Beran et al.(2013, Page 386) state long memory can be interpreted incorrectly as the presence of a trend when only 
simple diagnostic methods are used. Ould Haye \& Phillippe (2019) reverberate this statement as they explain the difficulty in distinguishing between long memory and non-stationarity when viewing the ACF plot as either condition exhibits similar behaviour. With the above in mind it seems plausible to believe that one could mistake long memory for non-stationarity and therefore turn to methods such as differencing to obtain a stationary form of the process. However, differencing is performed conventionally at integer amounts and therefore applying this technique to a fractionally integrated process will yield an overdifferenced series which can introduce undesirable aspects to the estimation procedure. For instance, generating forecasts for a large amount of steps ahead on an overdifferenced series will yield inferior estimates compared to those built with a model that is designed to handle the long memory characteristic as Geweke \& Porter Hudak (1983) state. With this being stated, one can see there is a need for statistical procedures that correctly distinguish between long memory and non-stationarity. Many well known methods exist for testing between stationarity and non-stationarity are available. However, very few procedures are available for testing long memory against non-stationarity. For instance, to our knowledge, the only published procedure that is currently available is Giraistis et al.(2006) as Ould-Haye \& Phillippe (2019) is forthcoming. Both of these procedures test for negative, short, or long memory under the null against a wide array of nonstationary behaviour under the alternative. The assumptions regarding the form of the process for each procedure are similar however, the test proposed by Giraistis et 
al.(2006) is derived under the time domain whereas Ould Haye \& Phillippe (2019) is developed within the frequency domain. The proposed test statistic within Giraistis et al.(2006) has a limiting distribution under the null which has a complicated form which involves stochastic integration of a fractional Brownian bridge and therefore, is difficult to obtain. The results from the simulation studies performed by the authors depict that the statistic experienced slightly undesirable results under the null as for a large array of simulated conditions the test appears to incur a minor bias. Moreover, for simulated conditions that were close to both the non-stationary and non-invertible boundaries, the statistic incurred high empirical size. An analogous simulation study was performed within Ould-Haye \& Phillippe (2019) which yielded an array of promising results. For instance, the statistic has a much simpler limiting distribution under the null hypothesis that is a weighted average of Chi-square random variables where the weights are explicitly defined and obtainable via numerical integration. Moreover, under the simulations conditions which used both Gaussian and non-Gaussian innovations, the statistic sparsely incurred any results that would allude to the existence of bias.

Given the promising results that we have reviewed from the two recent research endeavours by Gromykov et al.(2018) and Ould Haye \& Phillippe (2019), there appears to be research potential in developing statistical procedures within the frequency domain that will generate robust results. It can be seen that both procedures use the periodogram and the averaged periodogram to estimate the spectral density in con- 
structing their statistics. In respect to spectral density estimation, it is well known that the periodogram's estimation characteristics can be improved upon by techniques such as windowing, smoothing, or tapering. In the case of long and negative memory, tapering has been shown to enhance the periodogram's ability to estimate the spectral density under certain conditions. Some of the most prominent examples that depict this sentiment are Hurvich \& Beltrao (1993), Hurvich \& Ray (1995), Velasco (1999a), and Velasco (1999b). For instance, Hurvich \& Beltrao (1993) showed that tapering a negative memory process with the cosine bell taper reduces the asymptotic bias in the periodogram. Other authors such as Hurvich \& Ray (1995) have shown a reduction in bias in the periodogram ordinates when the underlying process is non-stationary or non-invertible by applying the cosine bell taper. Moreover, Velasco (1999a) and Velasco (1999b) showed that tapering the process with a cosine bell taper improved the estimator of the memory parameter $d$ in the presence of certain forms of nonstationarity. Therefore, given the results from the above it seemed rather intuitive to attempt to generate a set of periodogram-based tests that allow for the incorporation of tapering. Conventionally, tapering is known as applying a deterministic sequence of weights to a process to alter the properties of the periodogram as Priestely (1981) states. However, given the results from Csorgo et al.(2017) which showed that a random weighting scheme improved the estimation characteristics of the t-statistic pivot under short and long memory, it seemed of interest to develop a class of random weights to apply to the data in hopes of improving the asymptotic properties of the 
periodogram.

Overall, we produce an array of test statistics for two statistical procedures using the cosine bell taper and a certain class of weights which we denote as "random tapers". In the first procedure we implement both the cosine bell and the random taper whereas, in the second we only implement the cosine bell. The first procedure tests for short memory against the alternative of long memory. The second procedure tests for stationarity in the form of negative, short, or long memory against the presence of a trend. Both of these procedures are built within the frequency domain and require the use of the tapered periodogram and the averaged tapered periodogram. One of the primary hopes of our research is in regards to demonstrating the efficacy of performing inference within the frequency domain. We set out to accomplish this by showing that our tests yield results that improve upon certain aspects of corresponding statistical tests within the time domain. Therefore, we present the explicit forms of the asymptotic distribution under the null hypothesis, which are easily obtainable with conventional statistical software. Furthermore, we depict the precision in obtaining the correct theoretical size under the null of our statistics by evaluating the accrued P-value plots over an array of simulated conditions. Moreover, we demonstrate the stability in the window parameters that our statistics yield by assessing both Empirical Power and P-value plots. Through performing this we also demonstrate the ability for one to perform tapering with a set of random weights by presenting the sound theoretical and simulation results from the first procedure with the random 
taper applied. We now discuss the outline for how we present this research.

\subsection{Outline}

We first bring forth an assortment of theoretical concepts that assist the reader in grasping the underlying content within this research. These are then accompanied with a literature review where we depict the methodologies and results used within other relevant research. Upon completion of this we then proceed to present the theoretical content required for the derivation of the asymptotic distributions of our statistics. After this we describe the methodologies used for analyzing the performance of these statistics. Furthermore, we then present our findings section where we demonstrate the results from our simulation studies that were obtained via Monte Carlo methods with our proposed statistics for both of the procedures' null and alternate hypothesis'. We then accompany the first procedure's findings with the performance

of the statistics from Giraistis et al.(2003), Lobato \& Robinson (1998) and Gromykov et al.(2018). Upon achieving this we set out to implement two real world data sets within the test statistics from our two procedures to demonstrate the efficacy of these tests. Finally, we tie each individual section together by bringing to light the unifying themes within this research while simultaneously suggesting areas for further research within the conclusion. In the upcoming section we perform the theoretical review. 


\section{Theoretical Review}

\subsection{Time Series Processes}

A time series refers to a set of observations that are indexed by time and the methods developed with handling these processes are performed within the realm of time series analysis. Generally speaking, the statistical setting within time series analysis differs from that in the "classical" setting. For instance, neighbouring observations can be correlated with each other and therefore independence between observations will be lost. Within time series analysis there are two distinct domains in which provide a set of tools for drawing statistical inference. The first is the time domain which is primarily concerned with modelling the evolution of the series through information drawn from the correlation structure over time as Wei (1990) states. The second is the frequency domain which revolves around explaining the variance experienced within a series by harmonic components. Under certain conditions, these two approaches are mathematically equivalent yet the two domains offer different routes for examining the data. This link between the two domains is a relationship that is of fundamental importance to this research and will be further elaborated on when appropriate. We will now present selected concepts and definitions related to the time domain. 


\subsection{Time Domain}

Generally speaking, statistical endeavours conducted within the time domain are motivated by assuming the correlation between adjacent points in time is best explained in terms of the dependence of current values on past values of a process as Shumway \& Stoffer (2011) state. Some common areas of application of methods under the time domain are forecasting, determination of a transfer function, and dynamic modelling with multiple time series as Box et al.(2008) states. The ACVF is an important tool in time domain analysis as it helps depict the correlation that exists between neighbouring observations and therefore contains information on the relationship between past, current, and future values of the process. For a large class of stationary processes such as linear or Gaussian processes there exist further classifications that can be made in regards to the level of dependence within the correlation structure by analyzing the asymptotic behaviour of the ACVF as Giraistis et al.(2012, Page 35) state. An example of these characterizations are negative, short, and long memory. Within this research a process with negative, short, or long memory will have an ACVF that can be modelled by the following

$$
\gamma(h) \sim c_{\gamma}|h|^{-1+2 d}
$$

as $h \rightarrow \infty$ for $d \in(-.5, .5)$. From (2.1) it can be seen that the value of $d$ has implications on the rate or speed in which the ACVF dies down at as $h \rightarrow \infty$. The parameter $d$ is called the memory parameter as it refers to the amount of persistence or dependence embedded within the data. For instance, in $(2.1)$ with $d \in(0, .5)$, 
as $d$ increases the rate at decay of the ACVF towards zero declines. Moreover, the memory parameter $d$ is related to the classification of the memory structure of $X_{t}$ in such a way that if $X_{t}$ is of

- Short memory then $d=0$.

- Long memory then $0<d<.5$.

- Negative memory then $-.5<d<0$.

Interpretation in regards to the behaviour of the processes' correlation structure is available with the different memory structures. For instance, in the case of long memory (also known as long range dependence) the correlation between neighbouring observations dies down at a hyperbolic rate as they become farther apart. This slow decay in correlation caused by the high persistence embedded within the series causes the summation of the absolute values of the ACVF to be divergent as Giraistis et al.(2012) demonstrate. A process is known as having short memory (also known as short range dependence) if the correlation between neighbouring observations dies down quickly as observations become farther and farther spaced apart at a rate which allows the ACVF to be absolutely summable as Shumway \& Stoffer (2011) show. The following definition from Giraistis et al.(2012, Page 34, Definition 3.1.2) provides further information on the previous statements.

Definition 2.1. Let $X_{t}$ be a stationary process with ACVF $\gamma$. 
a.) If

$$
\sum_{h=-\infty}^{\infty}|\gamma(h)|<\infty, \quad \sum_{h=-\infty}^{\infty} \gamma(h)>0
$$

then the process is said to be short memory.

b.) If

$$
\sum_{h=-\infty}^{\infty}|\gamma(h)|=\infty
$$

then the process is said to be long memory.

c.) If

$$
\sum_{h=-\infty}^{\infty}|\gamma(h)|<\infty, \quad \sum_{h=-\infty}^{\infty} \gamma(h)=0
$$

then the process is said to be negative memory.

In the case of $(2.1)$ holding with $c_{\gamma}>0$ and $d \in(0, .5)$ the ACVF will experience slow hyperbolic decay asymptotically therefore, (2.3) is satisfied and the series is said to have long memory.

Moreover, in the case where (2.1) with $c_{\gamma}<0$ holds for some parameter $d \in$ $(-.5,0)$ and

$$
\sum_{h=\infty}^{\infty} \gamma(h)=0
$$

then (2.4) is satisfied and the process is said to have negative memory.

Within this research, our statistics utilize linear processes. We now present the following definition that elaborates on the characteristics of such processes. 
Definition 2.2. Let $\epsilon_{t}$ be a white noise process such that $\mathbb{E}\left(\epsilon_{t}\right)=0, \mathbb{E}\left(\epsilon_{t}\right)=\sigma_{\epsilon}^{2}<\infty$ and let $\psi_{k}, k=0,1, \ldots$. be a sequence of real numbers with

$$
\sum_{k=0}^{\infty} \psi_{k}^{2}<\infty
$$

We refer to a process $X_{t}$ as a linear process if it takes on the following form

$$
X_{t}=\sum_{k=0}^{\infty} \psi_{k} \epsilon_{t-k}
$$

With $X_{t}$ being a transformation of the innovations it will inherit their stationarity. Linear processes are of great interest to this research as they allow for the dependence structure of the process to be inferred from the rate at which the moving average coefficients $\psi_{k}$ decay at as Giraistis et al.(2012, Page 39) states. The following proposition from Giraistis et al.(2012, Page 39) elaborates on this.

Proposition 2.3. Let $X_{t}$ be defined as

$$
X_{t}=\sum_{k=0}^{\infty} \psi_{k} \epsilon_{t-k}
$$

Where $\epsilon_{t}$ are i.i.d with $\mathbb{E}\left(\epsilon_{t}\right)=0, \mathbb{E}\left(\epsilon_{t}^{2}\right)=\sigma_{\epsilon}^{2}, \mathbb{E}\left(\epsilon_{t}^{4}\right)<\infty$ and $\sum_{k=0}^{\infty} \psi_{k}^{2}<\infty$.

a.) If

$$
\sum_{k=0}^{\infty}\left|\psi_{k}\right|<\infty, \quad \sum_{k=0}^{\infty} \psi_{k} \neq 0
$$

then $X_{t}$ is said to be short memory.

b.) If

$$
\psi_{k} \sim c_{\psi} k^{-1+2 d}, k \rightarrow \infty
$$


with $d \in(0, .5)$, then $X_{t}$ is said to be long memory.

c.) If

$$
\psi_{k}=c_{\psi} k^{-1+2 d}\left(1+O\left(k^{-1}\right)\right), k \geq 1, c_{\psi} \neq 0
$$

and

$$
\sum_{k=0}^{\infty} \psi_{k}=0, \quad \sum_{k=0}^{\infty}\left|\psi_{k}\right|<\infty
$$

with $d \in(-.5,0)$, then $X_{t}$ is said to be negative memory.

Proof. The reader is asked to turn to Giraistis et al.(2012) for reference.

The methods we have presented previously in this section are all conducted within the time domain. However, as we have stated previously there exists another domain in which allows for statistical methods to be conducted. We now elaborate on this other domain which is referred to as the frequency domain.

\section{$2.3 \quad$ Frequency Domain}

Frequency domain analysis chooses to represent and study the variations of a time series with periodic components rather than the evolution of the process over time as Priestley (1981) state. As Shumway \& Stoffer (2011) claim one of the fundamental objectives of frequency domain analysis is in regards to identifying the dominant periodic components of a series and the frequencies in which they occur at. One function that is used to help perform this is called the spectral density. The spectral 
density describes how the variance of the process is distributed over the periodic components. A function that is typically used within the computations of estimating the spectral density is the discrete Fourier transform (DFT). We define the DFT of the process $X_{t}$ evaluated at the "jth" Fourier frequency $\omega_{j}=2 \pi j / n$, where $j$ is fixed, to be

$$
D\left(\omega_{j}\right)=\frac{1}{\sqrt{2 \pi n}} \sum_{t=1}^{n} X_{t} e^{-i t \omega_{j}} .
$$

Where its inverse Fourier transform is

$$
X_{t}=\sqrt{\frac{2 \pi}{n}} \sum_{j=1}^{n} D\left(\omega_{j}\right) e^{i \omega_{j} t} .
$$

The DFT is used to generate the periodogram which is a function that is generally chosen as a natural estimator of the spectral density is the periodogram given its intuitive form as Priestely (1981) states. The derivation of the periodogram $I_{n}\left(\omega_{j}\right)$ at the "jth" Fourier frequency is defined as the squared modulus of the DFT and is depicted below

$$
I_{n}\left(\omega_{j}\right)=\left|D\left(\omega_{j}\right)\right|^{2} .
$$

We will revisit the periodogram later in this research once we have presented the relationship between the time domain and frequency domain. More on this relation will be elaborated on now. 


\subsection{Two Approaches to Assessing the Characteristics of a Time Series}

Under certain conditions the time domain and frequency domain are mathematically equivalent as Giraistis et al.(2012) state. For instance, in the case of measuring the dependence of a process of a Gaussian or linear process a simple way of describing the dependence can be achieved in either the frequency domain or time domain via analyzing second-order characteristics. Similar to the sentiment within Wei (1990), one of the beneficial factors of a situation where either domain can be used for a statistical problem is that one domain might offer methods that are more convenient in some manner. One of the pinnacle theorems that allows one to depict the link between the two domains is achieved through the Spectral Representation Theorem which is presented now. We note that this theorem is primarily from Priestley (1981, Page 246) but we have extended it to the case in which $X_{t}$ is discrete which is similar to the style of Giraitis et al.(2012). The Spectral Representation Theorem states that given a zero-mean stationary stochastic process $X_{t}$, there exists a complex valued process $Z(\omega)$ with orthogonal increments, such that $X_{t}$ may be written as

$$
X_{t}=\int_{-\pi}^{\pi} e^{i t \omega} d Z(\omega) .
$$

Furthermore, $d Z(\omega)$ carries the following properties.

1. $\mathbb{E}(d Z(\omega))=0, \forall \omega$ 
2. $\mathbb{E}\left(|d Z(\omega)|^{2}\right)=d F(\omega)$, where $F(\omega)$ is called the spectral distribution of $X_{t}$,

3. $\mathbb{E}\left(d Z(\omega) \overline{d Z\left(\omega^{\prime}\right)}\right)=0, \quad \forall \omega \neq \omega^{\prime}$.

One of the concepts to take away from the above is that any zero mean stationary process with ACVF $\gamma$ will guarantee the existence of a unique spectral distribution function $F$ as Shumway \& Stoffer (2011, Page 182) state. This is depicted through the derivation of the ACVF using the Spectral Representation as

$$
\begin{aligned}
\gamma(h) & =\mathbb{E}\left(X_{t} X_{t-h}\right)=\int_{-\pi}^{\pi} \int_{-\pi}^{\pi} e^{i t \omega} e^{-i(t-h) \omega^{\prime}} \mathbb{E}\left(d Z(\omega) d^{*} Z\left(\omega^{\prime}\right)\right) \\
& =\int_{-\pi}^{\pi} e^{i t \omega-i t \omega+i h \omega} E\left(|d Z(\omega)|^{2}\right), \quad\left(\text { As } \mathbb{E}\left(d Z(\omega) \overline{d Z\left(\omega^{\prime}\right)}\right)=0, \omega \neq \omega^{\prime}\right) \\
& =\int_{-\pi}^{\pi} e^{i h \omega} d F(\omega) .
\end{aligned}
$$

The spectral distribution $F(\omega)$ can be interpreted as a culmulative distribution function $(\mathrm{CDF})$ not of probabilities but of the variances associated with the frequency $\omega$ as Shumway and Stoffer (2011, Page 181) state. Under additional assumptions of the process, $F(\omega)$ will be continuously differentiable which allows one to state $d F(\omega)=f(\omega) d \omega$ where $f$ is the spectral density. In this case we have

$$
\gamma(h)=\int_{-\pi}^{\pi} e^{i h \omega} d F(\omega)=\int_{-\pi}^{\pi} e^{i h \omega} f(\omega) d \omega .
$$

It should be noted that certain behaviour depicted in the ACVF gives rise to the existence of the spectral density. The following is from Giraistis et al.(2012, Page 11, Proposition 2.1.1) and sheds more light on this. 
Proposition 2.4. If a process has an ACVF that is square summable,

$$
\sum_{h=-\infty}^{\infty}(\gamma(h))^{2}<\infty
$$

then it has spectral density $f \in L_{2}[-\pi, \pi]$ that can be expressed with the Fourier Series

$$
f(\omega)=\frac{1}{2 \pi} \sum_{h=-\infty}^{\infty} \gamma(h) e^{-i \omega h},
$$

where $\omega \in[-\pi, \pi]$.

The above depicts that if the ACVF is square summable, the information conveyed within ACVF can be expressed through the spectral density as the ACVF and the spectral density form a Fourier pair. In this situation when inference is conducted on the second-order moments within the time domain the ACVF is used whereas, the spectral density is used within the frequency domain as Giraistis et al.(2012, Page 34) states.

Although square summability guarantees the existence of the spectral density, further assumptions on the form of the spectral density are required for the memory structure to be analyzed through either domain. An assumption that allows for this is in regards to the class of spectral densities which are of a semi-parametric form given below

$$
f(\omega)=|\omega|^{-2 d} f^{*}(\omega),
$$

where $\omega \in[-\pi, \pi]$ and $d \in(-.5, .5)$. Moreover, the function $f^{*}$ is assumed to be positive, even, and continuous function over $[-\pi, \pi]$ and bounded away from 0 . 
The spectral densities that satisfy the above are referred to as semi-parametric as Beran et al. (2013, Page 389) states because no assumptions on the shape of $f *$ outside an arbitrarily small neighbourhood of the origin are made. A spectral density $f(\omega)$ that satisfies $(2.6)$ behaves like $c_{f}|\omega|^{-2 d}$, as $\omega \rightarrow 0$, where $c_{f}$ is fixed and $\omega \in[-\pi, \pi]$. Hence, when $j$ is fixed, a semi-parametric spectral density has the following behaviour

$$
f\left(\omega_{j}\right) \sim c_{f}\left|\omega_{j}\right|^{-2 d}, \quad \text { as } n \rightarrow \infty
$$

for $d \in(-.5, .5)$. The above demonstrates that near the origin the spectral density takes on a certain limiting form. As one will note in (2.7) the term $c_{f}$ is fixed and therefore, as $\omega_{j} \rightarrow 0$, the behaviour the function exhibits is contingent on the value of $d$ and hence we can judge the memory structure of the process on this behaviour that the spectral density exhibits at the origin. Moreover, when (2.7) holds, $X_{t}$ is said to have negative memory, short memory, or long memory whether $d \in(-.5,0), d=0$, or $d \in(0, .5)$, respectively as Giraistis et al.(2012, Page 36) state.

It can be seen simply that a linear process that satisfies Proposition (2.3) will yield a square summable ACVF. Moreover, when a linear process is assumed to take on this form in conjunction with a spectral density $f$ that is semi-parametric, it allows one to assess the processes memory structure through either domain in view of the value of $d$. This is performed by evaluating either the asymptotic behaviour with the ACVF which is depicted in (2.1) for the time domain, or the behaviour of the spectral density at the origin which is depicted in (2.7) for the frequency domain. In the case 
of long memory where $d \in(0, .5)$ one will note that the spectral density will diverge at the origin whereas, in the case of negative memory where $d \in(-.5,0)$, the spectral density will decay to zero at the origin.

With the above in mind, we now proceed to present further content related to the periodogram.

\subsection{Periodogram}

Similar to the relationship that the ACVF and the spectral density bear, the periodogram and the sample ACVF hold a similar relationship as one can see in the following.

$$
I_{n}\left(\omega_{j}\right)=\frac{1}{2 \pi} \sum_{h=1-n}^{n-1} \gamma \hat{(h)} e^{-i h \omega_{j}}
$$

where

$$
\gamma(h)=\frac{1}{n} \sum_{t=1}^{n-|h|}\left(X_{t}-\bar{X}\right)\left(X_{t+|h|}-\bar{X}\right) .
$$

Hence,

$$
\mathbb{E}\left(I_{n}\left(\omega_{j}\right)\right)=\frac{1}{2 \pi} \sum_{h=1-n}^{n-1}\left(1-\frac{|h|}{n}\right) \gamma(h) e^{-i h \omega_{j}}
$$

One can note from the above that $\mathbb{E}\left(I_{n}(\omega)\right) \neq f(\omega)$. One can understand this bias by viewing the following alternative derivation of the periodogram's mean which will 
allow us to also refer to the reasoning behind methods that aim to reduce this bias.

$$
\begin{aligned}
\mathbb{E}\left(I_{n}(\omega)\right) & =\mathbb{E}\left(\frac{1}{2 \pi n} \sum_{t=1}^{n} \sum_{s=1}^{n} X_{s} X_{t} e^{-i(t-s) \omega}\right) \\
& =\frac{1}{2 \pi n} \sum_{t=1}^{n} \sum_{s=1}^{n} \int_{-\pi}^{\pi} \int_{-\pi}^{\pi} e^{i t \theta} e^{-i s \theta^{\prime}} e^{-i(t-s) \omega} \mathbb{E}\left(d Z(\theta) \overline{d\left(\theta^{\prime}\right)}\right) \\
& =\frac{1}{2 \pi n} \sum_{t=1}^{n} \sum_{s=1}^{n} \int_{-\pi}^{\pi} e^{i(t-s) \theta} e^{-i(t-s) \omega} \mathbb{E}\left(|d Z(\theta)|^{2}\right) \\
& =\int_{-\pi}^{\pi} \frac{1}{2 \pi n} \sum_{t=1}^{n} \sum_{s=1}^{n} e^{i(t-s)(\theta-\omega)} f(\theta) d \theta \\
& =\int_{-\pi}^{\pi} \frac{1}{2 \pi n}\left|\sum_{t=1}^{n} e^{i t(\theta-\omega)}\right|^{2} f(\theta) d \theta \\
& =\int_{-\pi}^{\pi} K_{n}(\theta-\omega) f(\theta) d \theta .
\end{aligned}
$$

Where the Fejér kernel is given by

$$
\left|K_{n}(\theta)\right|^{2}=\frac{1}{2 \pi n}\left|\sum_{t=1}^{n} e^{i \theta t}\right|^{2}=\frac{1}{2 \pi n}\left(\frac{\sin (n \theta / 2)}{\sin (\theta / 2)}\right)^{2}
$$

As one can see that the expected value of the periodogram is the convolution between the Fejér kernel and the spectral density. This convolution with the Fejér kernel causes the variance to transfer from other regions of the spectrum to $\omega$ as Schabenberger \& Gotway (2004, Page 196) show. This bias is known as leakage as the variance contribution of certain periodic frequencies "leaks" into neighbouring frequencies as Priestely (1981) states. Leakage induces undesirable aspects into the estimation procedure. An example of a situation where leakage arises is when the spectral density has a large dynamic range as Shumway and Stoffer (2011, Page 207) state. This large dynamic range affects the frequencies where the spectral density is 
small in particular as Schabenberger \& Gotway (2004, Page 196) state. It is important to note that in certain situations leakage can be nullified asymptotically. For instance, underneath short memory the periodogram is an asymptotically unbiased estimator of the spectral density as Priestely (1981, Page 418) states

$$
\mathbb{E}\left(I_{n}(\omega)\right)=\int_{-\pi}^{\pi} K_{n}(\theta-\omega) f(\theta) d \theta=f(\omega)+O\left(\frac{\log n}{n}\right) .
$$

However, under negative memory or long memory the periodogram ordinates are no longer asymptotically unbiased for estimating the spectral density as Hurvich \& Beltrao (1993) show. Furthermore, the authors speculate that this is due to the enormous spectral range in which the spectral density experiences under these conditions.

Overall, the phenomena of leakage calls for methods which improve the properties of the periodogram for estimating the spectral density. One technique that alters the periodogram in a way that aims to mitigate leakage via bias reduction is tapering as Schabenberger \& Gotway (2004, Page 196) state. We now further explain tapering.

\subsection{Tapering}

Tapering is a method that involves multiplying a series $X_{t}$ by a deterministic sequence of weights $h_{t}$ to reduce the bias in the periodogram by means of leakage reduction as Hurvich \& Beltrao (1993) state. This sequence of deterministic weights are chosen to abide by certain properties that alter the characteristics of the periodogram. For instance, the statements from Schabenberger \& Gotway (2004, Page 197) and Priestely (1981, Page 560) allow one to see that the goal of tapering is to replace the 
Fejér kernel $\left|K_{n}(\theta)\right|^{2}$ by a function $\left|H_{n}(\theta)\right|^{2}$, such that $\left|H_{n}(\theta)\right|^{2}$ reduces leakage more so than the Fejér kernel.

To depict how tapering introduces a new function in place of the Fejér kernel and alters the properties of the estimation procedure, we derive the expected value of the tapered periodogram for a zero mean stationary process with a square summable ACVF. Before doing so we note that the tapered periodogram is of the following form

$$
I_{n}^{T}\left(\omega_{j}\right)=\frac{\left|\sum_{t=1}^{n} h_{t} X_{t} e^{-i \omega_{j} t}\right|}{2 \pi \sum_{t=1}^{n} h_{t}^{2}} .
$$

We now present the previously referred to derivation in the following

$$
\begin{aligned}
\mathbb{E}\left(I_{n}^{T}(\omega)\right) & =\mathbb{E}\left(\frac{1}{2 \pi \sum_{t=1}^{n} h_{t}^{2}} \sum_{t=1}^{n} \sum_{s=1}^{n} h_{s} X_{s} h_{t} X_{t} e^{-i(t-s) \omega}\right) \\
& =\frac{1}{2 \pi \sum_{t=1}^{n} h_{t}^{2}} \sum_{t=1}^{n} \sum_{s=1}^{n} \int_{-\pi}^{\pi} \int_{-\pi}^{\pi} h_{t} h_{s} e^{i t \theta} e^{-i s \theta^{\prime}} e^{-i(t-s) \omega} \mathbb{E}\left(d Z(\theta) \overline{d Z\left(\theta^{\prime}\right)}\right) \\
& =\frac{1}{2 \pi \sum_{t=1}^{n} h_{t}^{2}} \sum_{t=1}^{n} \sum_{s=1}^{n} \int_{-\pi}^{\pi} h_{t} h_{s} e^{i(t-s) \theta} e^{-i(t-s) \omega} \mathbb{E}\left(|d Z(\theta)|^{2}\right) \\
& =\int_{-\pi}^{\pi} \frac{1}{2 \pi \sum_{t=1}^{n} h_{t}^{2}} \sum_{t=1}^{n} \sum_{s=1}^{n} h_{t} h_{s} e^{i(t-s)(\theta-\omega)} f(\theta) d \theta \\
& =\int_{-\pi}^{\pi} \frac{1}{2 \pi \sum_{t=1}^{n} h_{t}^{2}}\left|\sum_{t=1}^{n} h_{t} e^{i t(\theta-\omega)}\right|^{2} f(\theta) d \theta \\
& =\int_{-\pi}^{\pi}\left|H_{n}(\theta-\omega)\right|^{2} f(\theta) d \theta,
\end{aligned}
$$

where

$$
\left|H_{n}(\theta)\right|^{2}=\frac{1}{2 \pi \sum_{t=1}^{n} h_{t}^{2}}\left|\sum_{t=1}^{n} h_{t} e^{-i \theta t}\right|^{2} .
$$

With the above depicting how tapering alters the properties of the periodogram, we now proceed into a theorem which is required in order to state the asymptotic properties of the periodogram underneath both short and long memory conditions. 
Theorem 2.5. Let $z_{n t, j}, j=1, \ldots, s$ be $j$ arrays of real weights and $X_{t}$ be a of the form satisfying Proposition (2.3). Let $W_{n, j}=\sum_{t=1}^{n} z_{n t, j} X_{t}$. Assume that $\operatorname{Var}\left(W_{n, j}\right)=$ $\sigma_{W, j, n}^{2}{ }^{2}$ satisfies one of the three conditions

- (i) $\max _{1 \leq t \leq n}\left|z_{n t, j}\right|=o\left(\sigma_{W, j, n}\right)$ and $\sum_{t=1}^{n} z_{n t, j}^{2} \leq C \sigma_{W, j, n}^{2}$.

- (ii) $\max _{1 \leq t \leq n}\left|z_{n t, j}\right|=o\left(\sigma_{W, j, n}\right)$ and $\sum_{k=0}^{\infty}\left|\psi_{k}\right|<\infty$ Where $\psi_{k}$ are the moving average coefficients of $X_{t}$.

- (iii) $\left|z_{n 1, j}\right|+\left|z_{n n, j}\right|+\sum_{t=2}^{n}\left|z_{n t, j}-z_{n t, j-1}\right|=o\left(\sigma_{W, j, n}\right)$.

For $j=1, . ., s, k=1, \ldots, s$ and some $s$ by $s$ positive definite matrix $\Sigma_{s, s}$,

$$
\operatorname{Cov}\left(\frac{W_{n, j}}{\sigma_{W, j, n}}, \frac{W_{n, k}}{\sigma_{W, k, n}}\right)_{\{j, k\}} \longrightarrow \Sigma_{s, s}
$$

Then

$$
\left(\frac{W_{n, 1}}{\sigma_{W, 1, n}}, \ldots \frac{W_{n, s}}{\sigma_{W, s, n}}\right) \stackrel{\mathbb{D}}{\longrightarrow} N_{s}\left(0, \Sigma_{s, s}\right) .
$$

Proof. The proof has been omitted and the reader is asked to turn to Theorem 4.3.2 from Giraitis et al. (2012, Page 70, Theorem 4.3.2) for required referencing.

This theorem has been taken from Giraitis et al.(2012, Page 70, Theorem 4.3.2) and is the multivariate extension of the classical Lindenberg Central Limit Theorem to the case of dependency. We note that underneath the Lindenberg Central Limit Theorem the array of random variables needs to be independent with variances of each variable being finite and a third condition referred to as the Lindenberg Condition 
stated in our Appendix A as Definition (9.2). The importance of the Lindenberg Central Limit Theorem is that it offers an alternative route to the classical Central Limit Theorem to solve for the asymptotic distribution of a weighted summation of independent random variables that are not identically distributed. Furthermore, the value of this theorem from Giraitis et al.(2012) is that it allows one to solve for the limiting distribution of weighted sums of certain linear processes that are of negative, short, or long memory.

It should be noted from the above that condition (i), (iii) are useful for long memory processes. Whereas, condition (ii) is useful for short and negative memory.

With the above concepts in mind we now proceed to the literature review section.

\section{Literature Review}

Within this section we review some of the findings other authors that are relevant to this research. We begin by discussing an estimator of the memory parameter $d$.

\subsection{Log-Periodogram Regression}

It was Geweke \& Porter-Hudak (1983) that suggested a semi-parametric method for estimating the memory parameter $d$ which is known as the GPH estimator. This procedure was based off of performing a regression using the log of the periodogram 
with the following equation as Sibbertsen \& Busch (2018) state

$$
\log I_{n}\left(\omega_{j}\right)=C+d v_{j}+\epsilon_{j}, \quad \text { where } v_{j}=-\log 2-2 \cos \omega_{j}, \quad j=1, \ldots, m .
$$

Where $C$ is constant and $\epsilon_{j}$ is the error term. The GPH estimator is the LSE of $d$ in the regression above. The explicit form of the GPH estimator is given in the following

$$
d_{G P H}=\sum_{j=1}^{m} \frac{1}{2} \frac{\left(Y_{j}-\bar{Y}\right) \log I_{n}\left(\omega_{j}\right)}{\sum_{j=1}^{m}\left(Y_{j}-\bar{Y}\right)^{2}} .
$$

where $Y_{j}=\log \left|2 \sin \frac{\omega_{j}}{2}\right|$ and $\bar{Y}=\frac{1}{m} \sum_{j=1}^{m} Y_{j}$ and $\omega_{j}=\frac{2 \pi j}{n}$ for $j=1, \ldots ., m$. Here

$m$ is called the bandwidth parameter and is chosen so that $\frac{m}{n} \rightarrow 0$, as $n, m \rightarrow \infty$. The choice of $m$ creates a balancing effect between the bias and variance reduction as Beran et al. (2013, Page 442) state. In regards to the asymptotic distribution of the $d_{G P H}$ estimator it was Robinson (1995) found that for semi-parametric models when $d \in(-.5, .5)$ then $\sqrt{m}\left(d_{G P H}-d\right) \stackrel{\mathbb{D}}{\longrightarrow} N\left(0, \frac{\pi^{2}}{24}\right)$. Therefore, showing the estimators consistency. We now speak of the work of Hurvich \& Beltrao (1993) which is related to the statistics we have developed.

\subsection{Distribution of Periodogram Ordinates}

Hurvich \& Beltrao (1993) considered the asymptotic distribution of the normalized periodogram ordinates at lower Fourier frequencies of a zero mean, stationary Gaussian process with negative, short, and long memory and a semi-parametric spectral density with $d \in(-.5, .5)$. The authors showed that under long or negative memory the periodogram ordinates suffer from positive asymptotic relative bias (ARB). 
The authors solve for the ARB of the normalized periodogram and express it in the following form

$$
\lim _{n \rightarrow \infty} \mathbb{E}\left(\frac{I_{n}\left(\omega_{j}\right)}{f\left(\omega_{j}\right)}\right)=L_{j}(d)=\frac{2}{\pi} \int_{-\infty}^{\infty} \frac{\sin ^{2}(\theta / 2)}{(2 \pi j-\theta)^{2}}\left|\frac{\theta}{2 \pi j}\right|^{-2 d} d \theta .
$$

Within their research, the authors break down the periodogram into components in the following way

$$
D\left(\omega_{j}\right)=\frac{1}{2 \pi n} \sum_{t=1}^{n} X_{t} e^{-i \omega_{j} t}=A\left(\omega_{j}\right)-i B\left(\omega_{j}\right) .
$$

Where

$$
A\left(\omega_{j}\right)=\frac{1}{2 \pi n} \sum_{t=1}^{n} X_{t} \cos \left(\omega_{j}\right), \quad B\left(\omega_{j}\right)=\frac{1}{2 \pi n} \sum_{t=1}^{n} X_{t} \sin \left(\omega_{j}\right) .
$$

The authors showed that the asymptotic form of the second order moments of the components are as follows

$$
\begin{gathered}
\lim _{n \rightarrow \infty} \operatorname{Cov}\left(\frac{A\left(\omega_{k}\right)}{\sqrt{f\left(\omega_{k}\right)}}, \frac{A\left(\omega_{j}\right)}{\sqrt{f\left(\omega_{j}\right)}}\right)=L_{j, k}(d)-R_{j, k}(d), \\
\lim _{n \rightarrow \infty} \operatorname{Cov}\left(\frac{B\left(\omega_{k}\right)}{\sqrt{f\left(\omega_{k}\right)}}, \frac{B\left(\omega_{j}\right)}{\sqrt{f\left(\omega_{j}\right)}}\right)=L_{j, k}(d)+R_{j, k}(d), \\
\lim _{n \rightarrow \infty} \operatorname{Cov}\left(\frac{B\left(\omega_{k}\right)}{\sqrt{f\left(\omega_{k}\right)}}, \frac{A\left(\omega_{j}\right)}{\sqrt{f\left(\omega_{j}\right)}}\right)=0 \\
\lim _{n \rightarrow \infty} \operatorname{Var}\left(\frac{A\left(\omega_{j}\right)}{\sqrt{f\left(\omega_{j}\right)}}\right)=\frac{1}{2} L_{j}(d)-R_{j}(d), \lim _{n \rightarrow \infty} \operatorname{Var}\left(\frac{B\left(\omega_{j}\right)}{\sqrt{f\left(\omega_{j}\right)}}\right)=\frac{1}{2} L_{j}(d)+R_{j}(d) .
\end{gathered}
$$

Where

$$
R_{j, k}(d)=\frac{\left|(j k)^{d}\right|}{\pi} \int_{-\infty}^{\infty} \frac{\sin ^{2}(\theta / 2)}{(2 \pi j-\theta)(2 \pi k+\theta)}\left|\frac{\theta}{2 \pi}\right|^{-2 d} d \theta,
$$




$$
\begin{gathered}
L_{j, k}(d)=\frac{\left|(j k)^{d}\right|}{\pi} \int_{-\infty}^{\infty}\left[\frac{\sin ^{2}(\theta / 2)}{(2 \pi j-\theta)(2 \pi k-\theta)}\left|\frac{\theta}{2 \pi}\right|^{-2 d} d \theta,\right. \\
R_{j}(d)=\frac{1}{\pi} \int_{-\infty}^{\infty} \frac{\sin ^{2}(\theta / 2)}{(2 \pi j-\theta)(2 \pi j+\theta)}\left|\frac{\theta}{2 \pi j}\right|^{-2 d} d \theta .
\end{gathered}
$$

Furthermore, the authors show that the components of the periodogram converge in distribution to normal random variables .

$$
\frac{1}{\sqrt{\left(\frac{L_{j}(d)}{2}-R_{j}(d)\right)}} \frac{A\left(\omega_{j}\right)}{\sqrt{f\left(\omega_{j}\right)}} \stackrel{\mathbb{D}}{\longrightarrow} Z_{1}^{U T}(j), \quad \frac{1}{\sqrt{\left(\frac{L_{j}(d)}{2}+R_{j}(d)\right)}} \frac{B\left(\omega_{j}\right)}{\sqrt{f\left(\omega_{j}\right)}} \stackrel{\mathbb{D}}{\longrightarrow} Z_{2}^{U T}(j) .
$$

Where $Z_{1}^{U T}(j), Z_{2}^{U T}(j)$ are the random variables given in Hurvich \& Beltrao (1993).

\subsection{Ramifications of Implementing the Cosine Bell Taper}

In response to the findings given above, the authors in Hurvich \& Beltrao (1993) found that applying a cosine bell taper to the series when $d \neq 0$ substantially reduced the ARB except at the first Fourier frequency where the ARB increased dramatically for values of $d$ close to .5. However, looking at the authors' results one can see that even at the first Fourier frequency, values of $-.5<d<.1$ are improved in terms of ARB when tapering with the cosine bell. Overall, for the first Fourier frequency tapering eliminates the bias when $d$ is close to -.5 and shifts it towards $d=.5$.

In the case of short memory there is an important distinction to make between the components of the tapered periodogram from those of the untapered periodogram as one will note, components are now asymptotically correlated with their neighbours at 
neighbouring Fourier frequencies. This is because by applying the cosine bell taper each DFT becomes a linear combination of neighbouring DFT's spaced one frequency apart which can be visualized in the following equation

$$
\frac{1}{\sqrt{12}}\left(2 D\left(\omega_{j}\right)-D\left(\omega_{j+1}\right)-D\left(\omega_{j-1}\right)\right) .
$$

This is due to the fact that

$$
h_{t} e^{-i \omega_{j} t}=\frac{1}{2}(1-\cos (2 \pi t / n)) e^{-i \omega_{j} t}=\frac{1}{2}\left(1-\frac{1}{2}\left(e^{-i 2 \pi t / n}+e^{-i 2 \pi t / n}\right)\right) e^{-i \omega_{j} t} .
$$

Hence,

$$
\begin{aligned}
D_{T}\left(\omega_{j}\right) & =\frac{2}{\sqrt{3 \pi n}} \sum_{t=1}^{n} h_{t} X_{t} e^{-i \omega_{j} t} \\
& =\frac{1}{\sqrt{3 \pi n}} \sum_{t=1}^{n} X_{t} e^{-i \omega_{j} t}-\frac{1}{2} \frac{1}{\sqrt{3 \pi n}} \sum_{t=1}^{n}\left(e^{-i(2 \pi t / n)}+e^{i(2 \pi t / n)}\right) X_{t} e^{-i \omega_{j} t} \\
& =\frac{1}{\sqrt{3 \pi n}} \sum_{t=1}^{n} X_{t} e^{-i \omega_{j} t}-\frac{1}{2 \sqrt{3 \pi n}} \sum_{t=1}^{n} X_{t} e^{-i\left(\omega_{j}+(2 \pi t / n)\right) t}-\frac{1}{2 \sqrt{3 \pi n}} \sum_{t=1}^{n} X_{t} e^{-i\left(\omega_{j}-(2 \pi t / n)\right) t} \\
& =\frac{1}{\sqrt{12}}\left(2 D\left(\omega_{j}\right)-D\left(\omega_{j+1}\right)-D\left(\omega_{j-1}\right)\right) .
\end{aligned}
$$

Whereas in the untapered case, asymptotically the DFTs are uncorrelated with each other for a short memory series.

Further research was conducted on tapering with the cosine bell taper under a fractionally integrated Gaussian process with semi-parametric assumptions of the spectral density through Hurvich \& Ray (1995). For instance, the authors considered the asymptotic characteristics of the untapered periodogram and tapered periodogram when the process is non-invertible $(d \leq-.5)$ or non-stationary $(d \geq .5)$. The authors 


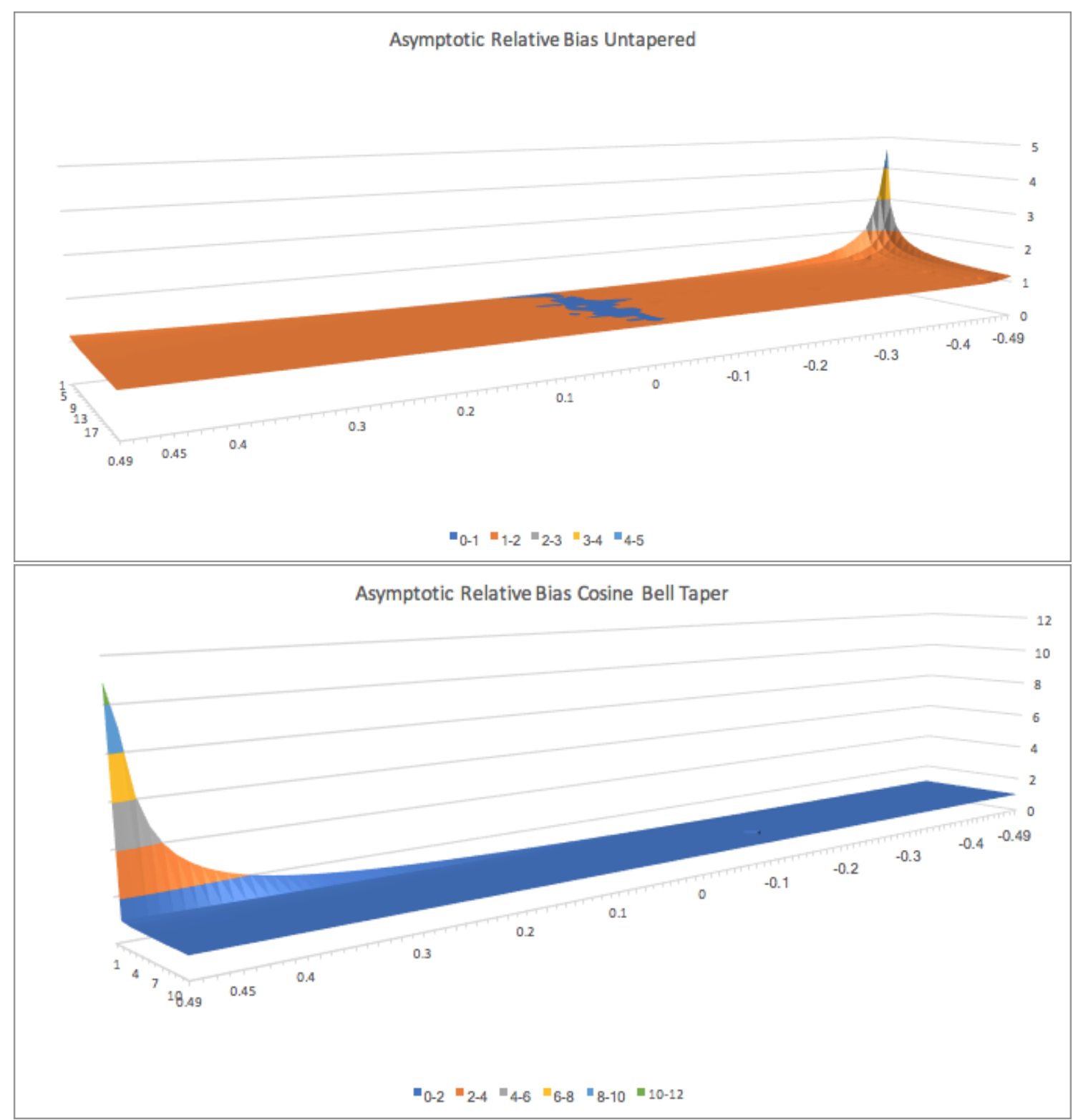

Figure 1: Asymptotic Relative Bias of Untapered and Tapered Periodogram

For the graphs above we have the $A R B$ at values of $d$ and $j$. The values of $d$ are on the front axis and stretch from .49 to -.49. On the left hand side axis we have the Fourier frequencies. 
find that the normalized untapered periodogram has a bounded first moment when $d \in[.5,1.5)$. They also found that the periodogram of either a non-invertible or nonstationary process suffers from ARB that depends on $j$ and that the GPH estimator is affected in both cases. By tapering with a cosine taper the authors show that when $d \in(-1,-.5)$ the ARB for the tapered periodogram is nearly zero. When $d>-.5$ the ARB is almost always less than the tapered periodogram for the periodogram itself. However, when $d$ is close to 1 the tapered periodogram ARB varies more with $\mathrm{j}$ than the periodogram of the untapered series. Therefore, the authors suggest that tapering may increase bias in the GPH estimators if the number of Fourier frequencies used is not large. Overall, the authors suggest using a taper to reduce the bias in the untapered periodogram for $d \in[.5,1.5)$. For the normalized tapered periodogram the authors showed that

$$
\mathbb{E}\left(\frac{I_{n}^{T}\left(\omega_{j}\right)}{f\left(\omega_{j}\right)}\right)=\infty .
$$

When $d \geq .5, j=1$ or $d \leq-2.5, j \geq 2$. Therefore, the authors implement the tapered periodogram into the GPH estimator and remove the first ordinate. The results they found were that for $d \geq .5$ the bias in the GPH estimator is reduced using the tapered periodogram with the first ordinate removed. Velasco (1999b) tested the consistency of the GPH estimator even further. For instance, the author showed that in the case of Gaussian process when $d<.75$ the GPH estimator is still asymptotically normal and is still consistent for $d<1$.

Velasco (1999b) also analyzed the behaviour of the log-periodogram regression 
estimators for the memory parameter and found that they are still consistent when the cosine bell taper is used in cases where the series departs from stationarity. For instance, the author showed that when $d<1.5$ the GPH estimator is still asymptotically normal and consistent.

Furthermore, Velasco (1999b) also found that for a non-stationary series, the GPH estimator based on the tapered data often has less bias, is more nearly invariant to first differencing, but has a larger variances than GPH estimator without tapering. The author also states that the tapered periodogram improves upon the asymptotic properties with respect to the untapered periodogram, because the tails of the kernel induced by the taper decrease much faster with the frequencies and the sample size than the tails of Fejer Kernel. Moreover, by applying the cosine bell taper one has the ability reduce the bias of the periodogram on the tails, even for frequencies close to a singularity and for non-integratable functions, if they are smooth enough as the author states.

Similarly, Sibbertsen (2001) showed that there is a strong reduction of the bias in the case of slow decaying deterministic trends and structural breaks when the tapering is used.

As one can see from the literature presented within this research it appears that overall there is evidence that tapering improves estimation procedures that use the periodogram through bias reduction. The sentiment in this statement is reflected in Beran et al.(2013, Page 458) as the author states the reduction in finite sample 
bias can be substantial by tapering the data for the semi-parametric estimator of the memory parameter. With the above in mind we now proceed to the next section where we review statistical procedures that test for certain characteristics of stationary processes.

\subsection{Time Domain Tests for Short v. Long Memory}

We now present relevant statistical methods that test for short or long memory under the time domain. We start by reviewing the development of these methods.

As Taqqu et al.(1999a) state the importance of long memory processes revolves around the fact that they provide an interpretation of what is known as "Hurst's Law" or the "Hurst Effect". Given a set of observations $\left\{X_{t}, t \geq 1\right\}$ with partial sums $Y_{n}=\sum_{t=1}^{n} X_{t}$ for $n \geq 1$ and sample variance $S_{n}=\frac{\sum_{t=1}^{n}\left(X_{t}-n^{-1} Y_{t}\right)^{2}}{n} n \geq 1$. The Rescaled Adjusted Range Statistic (R/S statistic) is defined as:

$$
\frac{R}{S}=\frac{1}{S_{n}}\left(\max _{0 \leq t \leq n}\left(Y_{t}-\frac{t}{n} Y_{n}\right)-\min _{0 \leq t \leq n}\left(Y_{t}-\frac{t}{n} Y_{n}\right)\right), \quad \text { for } n \geq 1
$$

As Taqqu et al.(1999a) state Hurst (1951) found that many real world phenomena appear to be well represented by the relation $\mathbb{E}\left(\frac{R}{S}\right) \sim C n^{H}$, as $n \rightarrow \infty$. Where $C$ is some positive constant and $H$ which is referred to as the "Hurst Parameter" and is defined on the interval $(0,1)$ as the Hurst Parameter is related to the memory parameter by $H=d+.5$, where $d \in(-.5, .5)$. The previously referred to relation was employed to model long memory time series. In the case of $X_{t}$ coming from a short memory process, then $\mathbb{E}\left(\frac{R}{S}\right) \sim C n^{5}$. As Taqqu et al.(1999a) state the 
discrepancy between these two relations is generally referred to as the "Hurst Effect". Classical R/S analysis which aims at inferring from a set of sampled observations the value of the Hurst parameter for the data generating process (DGP) that created the observed sample as Taqqu et al.(1999a) state. However, the authors note that classical R/S analysis is not very reliable in the case of small samples but yet can be useful as a graphical technique on larger samples. Some other issues with this type of classical R/S analysis is in regards to the lack of distribution theory for the underlying statistic given in (3.10) which stand in the way of allowing one to create a statistical test as Taqqu et al.(1999a) state in essence. However, Lo (1991) provided a modified version of the R/S statistic given in (3.10). Lo (1991) modified the R/S statistic by removing the $S_{n}$ in the denominator and replacing it by a consistent estimator of the square root of the variance of the partial sum of $Y_{n}$ as the authors in Willinger et al. (1999) states. This allowed for the derivation of a limiting distribution of the modified R/S statistic underneath the null hypothesis of short memory and the alternate of long memory. Lo (1991) showed promising results such as obtaining high empirical power with simulated data. The test devised by Lo(1991) received support from other authors within econometric literature. However, Taqque et al.(1999b) and Taqqu et al. (1999a) showed that there were a number of issues with the modified R/S statistic developed by Lo (1991). One of these is in regards to the strong preference the procedure has for failing to reject the null hypothesis as Taqqu et al.(1999b) state. This led Taqqu et al.(1999b) to caution others in regards to using the procedure 
proposed by Lo (1991) in isolation. Another critique of Lo's procedure was highlighted in Beran et al.(2013, Page 412) and is in regards to finding a suitable value for the window parameter $m$ where the short memory structure of the given data set is unknown. Another concern is given by Beran et al.(2013,Page 412) as the author states that this test is not robust when the series is non-stationary. For instance, Giraitis et al.(2001) showed that certain forms of non-stationarity (trend or change points), lead to a rejection of the null hypothesis of the R/S method. Therefore, indicating a flaw of the test and indicating the need for further testing to discriminate between non-stationarity and long memory as the authors state. Some other flaws include a bias towards rejecting long memory as Taqqu et al.(1999b) state.

As Beran et al.(2013) states a statistical test that is devised for the testing the stationarity of a time series is the KPSS statistic which was developed by Kwiatkowski et al.(1992). Under the null hypothesis of the KPSS test the time series is stationary around a deterministic trend against the alternative that the series contains a unit root. As Lee \& Schmidt (1996) state, under the null hypothesis, it is assumed that the process is of short memory. The authors went on to show that the test is robust asymptotically for testing the null of a short memory series against a long memory series. 


\subsubsection{V/S Statistic}

Although the KPSS statistic possessed desirable qualities it was Giraitis et al.(2003) who suggested a non-parametric procedure that assesses long memory for stationary processes. The authors compared this statistic with the modified RS statistic, LR statistic, and KPSS. The VS statistic is similar to both the KPSS and Modified RS statistic in form and therefore, all the statistics include the same window parameter $q$. There are notable improvements that this procedure's test statistic yields in comparison to other time domain procedures using the R/S statistic in some form. For example, the VS statistic is less sensitive to the choice of bandwidth parameter $q$ than the test based on the statistic from Lo (1991) as Giraistis et al.(2003) states. Also, the VS statistic has uniformly higher power than the KPSS test and test has performed more optimally in terms of a balance in empirical size and power as Giraitis et al.(2003) state. It should also be noted that in regards to the KPSS modified RS statistic, the VS statistic has a simpler limiting distribution under the null.

The V/S test statistic tests for short memory under the null against the alternative of long memory. For a short memory series $X_{t}$

$$
\frac{1}{n^{1 / 2}} \sum_{j=1}^{[n t]}\left(X_{j}-E\left(X_{j}\right)\right) \rightarrow \sigma \mathbb{W}(t),
$$

as $n \rightarrow \infty$. Where $\mathbb{W}(t), 0 \leq t \leq 1$ is an standard Wiener process. It should be noted that another assumption the VS statistic uses is in regards to the fourth order 
culmulant given below

$$
\sup _{h} \sum_{r, s=-\infty}^{\infty}|\kappa(h, r, s)|<\infty
$$

where $\kappa(h, r, s)$ is the fourth order culmulant. Underneath the null conditions given above $M_{n} \rightarrow U_{V / S}$

Where the test statistic is defined as $M_{n}=\frac{V_{n}}{\tilde{s}_{n, q}^{2}}$ with

$$
V_{n}=\frac{1}{n^{2}}\left(\sum_{k=1}^{n}\left(S_{k}^{*}\right)^{2}-\frac{1}{n}\left(\sum_{k=1}^{n} S_{k}^{*}\right)^{2}\right)
$$

and

$$
S_{k}^{*}=\sum_{j=1}^{k}\left(X_{j}-\overline{X_{n}}\right), \quad \tilde{s}_{n, q}^{2}=\tilde{\gamma}_{0}+2 \sum_{k=1}^{q}\left(1-\frac{k}{n}\right) \tilde{\gamma}_{k},
$$

where $\tilde{\gamma}$ is the sample autocovariances and $\overline{X_{n}}$ is the sample mean. The random variable $U_{V / S}$ can be explicitly represented as

$$
U_{V / S}=\frac{1}{4 \pi^{2}} \sum_{j=1}^{\infty} \frac{\left(Y_{2 j-1}^{2}+Y_{2 j}^{2}\right)}{j^{2}},
$$

where $Y_{2 j}$ is a standard normal independent random variable as Giraistis et al.(2003) state. Under the null hypothesis, the test statistic converges to a random variable with a non-degenerated distribution which takes on the following explicit form

$$
F_{U_{V / S}}=1+\sum_{k=1}^{\infty}(-1)^{k} e^{-2 k^{2} \pi^{2}}
$$

It should be noted that for the window parameter $q=q(n)$, such that $q=o(n)$.

The authors perform simulation studies using Monte Carlo simulations with the VS, KPSS, and Modified R/S statistic's with linear sequences of the form of an 
AR(1) stationary process with i.i.d standard normal innovations. The authors find that optimal value of $q$ depends on $\phi$ in addition to $n$. Moreover, it is shown that as the AR coefficient $\phi$ increases, larger values of the window parameter $q$ must be utilized to obtain for the correct theoretical size under the null. For instance, when $\phi \geq .5$ and lower order values of $q$ such as $q=0,1,2,5$. For the KPSS and the VS statistic the authors go on to show that for linear processes the VS statistic achieves somewhat of a better balance of size and power than the modified R/S and the KPSS tests and that the modified R/S test suffers from size distortion and very weak power for larger values of $q$. The authors also claim that the LR test distinguishes between short and long memory with a very high degree of precision and should be used in conjunction with the R/S type test. The authors also go on to state that although the LR statistic performs very well for linear and $\mathrm{ARCH}$ processes, its theoretical and empirical properties such as the automatic bandwidth selection, require additional investigation. Moreover, under the null and alternative hypothesis without assuming any particular structure of observations except stationarity, the authors state that the R/S, KPSS, and the VS tests are more useful than the LR. However, one of the limitations of the $\mathrm{V} / \mathrm{S}$ statistic is its high empirical size when $\phi \geq .5$ for moderate sample sizes as noted by Giraistis et al.(2003). We now present similar procedures that have been developed underneath the frequency domain. 


\subsection{Frequency Domain Tests for Short v. Long Memory}

\subsubsection{LR Statistic}

Within the frequency domain Lobato \& Robinson (1998) proposed a non-parametric test for short memory against the alternative of long memory. The test statistic takes on the following form

$$
t=\sqrt{m} \frac{\sum_{j=1}^{m} v_{j} I\left(\omega_{j}\right)}{\sum_{j=1}^{m} I\left(\omega_{j}\right)},
$$

where

$$
v_{j}=\log (j)-\frac{1}{m} \sum_{j=1}^{m} \log (j) .
$$

As Gromykov et al.(2018) state under certain conditions on the window parameter $m$ and assuming that the spectral density is twice boundedly differentiable near the origin and $d=0$, the test statistic given in (3.15) converges in distribution to a standard normal random variable. The authors showed that the LR test is quite efficient in achieving the correct empirical size as shown in their simulation studies.

\subsection{2 $\mathrm{Q}_{n, m}(\mathrm{~s})$ Statistic}

It was Gromykov et al.(2018) that developed a statistical procedure within the frequency domain that allowed for testing between short memory under the null against long memory within the alternate for linear processes that take on the form of Definition (2.3). As noted in Gromykov et al.(2018) the normalized periodogram is an asymptotically unbiased estimator of the spectral density under short memory. 
Moreover, the authors showed that the averaged periodogram over different blocks is a consistent estimator for the spectral density in the case of short memory. This method is known as the Bartlett or Welch Method depending if the observations within each block are non-overlapping or overlapping, respectively. The test statistic developed by the authors takes on the following form

$$
Q_{n, m}(s)=\sum_{j=1}^{s} \frac{I_{n}\left(\omega_{j}\right)}{\frac{1}{m} \sum_{k=1}^{m} I_{n, k}\left(\omega_{j}\right)}
$$

Where $s$ is the number of desired Fourier frequencies and $m=\frac{n}{l}$ is the number of blocks. Where $l=\frac{n}{m}$ is the size of each block. Both $m$ and $s$ are window parameters. However, as the authors note $s$ is fixed whereas, $m$ and consequently $l$ are both functions of $n$ such that both $m=m(n)$ and $l=l(n) \rightarrow \infty$ as $n \rightarrow \infty$. The following theorem is directly from Gromykov et al.(2018) which depicts the asymptotic distribution of the statistic underneath each hypothesis.

Theorem 3.1. Let $X_{t}$ satisfy the form of Proposition (2.3). Let $Q_{n, m}(s)$ be defined as in (3.17) where $s \geq 1$ is fixed and $m=o(n)$.

(a) If $X_{t}$ is a short memory process in the sense that $d=0$ and

$$
\sum_{j=1}^{\infty}\left|\psi_{j}\right|<\infty
$$

then

$$
Q_{n, m}(s) \stackrel{\mathbb{D}}{\longrightarrow} Q(s) .
$$

Where $Q(s)$ has a $\Gamma(s, 1)$ distribution where $\Gamma$ is a Gamma random variable. 
(b) If $X_{t}$ is long memory in a sense that $f$ is of the form of (2.6) with $d \in(0, .5)$, then

$$
Q_{n, m}(s) \stackrel{\mathbb{P}}{\longrightarrow} \infty .
$$

Proof. We ask the reader to refer to Gromykov et al.(2018) for desired referencing.

There are notable features of this procedure that possess desirable aspects. For instance, the limiting distribution of the test statistic under the null is $\Gamma(s, 1)$ which is simplistic and readily available within all statistical software as the authors note. The test statistic also preserves desirable empirical size for both Gaussian and nonGaussian innovations. The test statistic yields other desirable attributes in respect to other competing research. For instance, in comparing the structural form of the $Q_{n, m}(s)$ uses a blocking method whereas, the LR test estimates $f(0)$. As well as the $Q_{n, m}(s)$ test does not assume smoothness conditions of the spectral density near the origin, except in the continuity of $f$ in (2.6). The authors also note that another difference between their proposed test statistic and the LR statistic is that they do not make as strict assumptions on their bandwidth (window) parameters except that $l=o(n)$. Whereas the LR test relies on more stringent assumptions of their window parameter $m$. The authors conduct a numerical simulation study of their proposed test statistic to assess the performance in terms of empirical size of the $Q_{n, m}(s)$ test at differing values of $m$ and $s$. The authors then compare its results against those of the LR test and the VS test with the suggested window parameter values given in Lobato \& Robinson (1998) and Giraistis et al.(2003), respectively. The authors 
perform Monte Carlo simulation by simulating from independent AR(1) process with coefficient values of $\phi=(-.5, .5, .8)$ for sample sizes of $n=(1000,5000,10000)$ and innovations from standard normal, centered standard exponential, and centered standard log-normal and then compute each sample's P-value associated to the $Q_{n, m}$ test and then calculate the empirical cumulative distribution function of the P-values. The authors find that a potential optimal value of $m$ is $\sqrt{n}$ as it allows for the empirical size to be close to the theoretical size of the test for both Gaussian and non-Gaussian innovations. The authors note that the choice of $s$ depends upon the sample size. For instance, it can be seen that the empirical size seems to be increasing as $s$ increases. When comparing the $Q_{n, m}(s)$ test results against those of the LR and VS tests, the authors note that the VS and LR tests depict a lack of robustness in terms of empirical size precision in the case of non normal innovations whereas, their proposed test statistic exhibits more reasonable values of empirical size. In terms of power, the authors find that the empirical power of the test increases as $s$ increases for all tested innovation distributions. The LR and VS tests yield high empirical power compared to the $Q_{n, m}(s)$ test as the empirical sizes for these statistics were higher than the latter. Within the next section we review the existing procedures that test for stationarity with negative, short, or long memory against common forms of non-stationarity. 


\subsection{Testing for Dependence v. Non-Stationarity}

Within this section we review the statistical procedures used for testing the stationarity of linear processes.

\subsection{1 $\mathbf{T}_{n}(\mathrm{~d})$ Statistic}

It was Giraistis et al.(2006) who devised a procedure for testing a stationary process with a certain dependence structure against common forms of non-stationarity under the time domain. The test statistic within Giraistis et al.(2006) takes on the following form:

$$
T_{n}(\hat{d})=\left(\frac{q}{n}\right)^{2 \hat{d}} \frac{V_{n}}{s_{n, q}^{2}}
$$

where $q=q(n)=O\left(n^{.5}\right)$. Moreover,

$$
V_{n}=\frac{1}{n^{2}}\left(\sum_{k=1}^{n}\left(S_{k}^{*}\right)^{2}-\frac{1}{n}\left(\sum_{k=1}^{n} S_{k}^{*}\right)^{2}\right)
$$

and

$$
S_{k}^{*}=\sum_{j=1}^{k}\left(X_{j}-\overline{X_{n}}\right), \quad \tilde{s}_{n, q}^{2}=\tilde{\gamma}_{0}+2 \sum_{k=1}^{q}\left(1-\frac{k}{n}\right) \tilde{\gamma}_{k} .
$$

Under the null hypothesis $X_{t}=\mu+Y_{t}$. Where $Y_{t}$ is a zero mean stationary process with an autocovariance function $\gamma_{y}$ of the process $Y_{t}$ satisfies Definiton (2.1) and where $d \in\left[-a_{1}, a_{2}\right]$ with $0 \leq a_{1}, a_{2}<.5$. Under the null hypothesis the test statistic converges in distribution to a stochastic integral involving a fractional Brownian Bridge. 
Under the alternative hypothesis let $X_{t}=\mu+g_{n}(k)+Y_{t}$ or $X_{t}=\mu+g_{n}(k)+$ $X_{t-1}+Y_{t}$. Where $Y_{t}$ is defined as the same process in the null hypothesis except $|d|<.5$. Here $g_{n}$ is a deterministic trend that satisfies certain conditions that we have omitted for the sake of brevity.

The authors go on to perform an array of Monte Carlo simulations with Gaussian innovations for 5000 independent replications of conditions under the null and alternate hypothesis. The results the authors present are from the statistic with chosen values of the window parameters being $m=n^{7}$ and $q=n^{1 / 3}$. The results look to allude to a slight bias for the statistic for theoretical sizes of .05 and .1. when $d \in(-.2, .2)$, under the null hypothesis. However, the test looks to perform well yielding high empirical power under many of the tested alternative conditions. Moreover, the results from the simulation studies performed within Giraistis et al.(2006) were done exclusively with Gaussian innovations.

We now review the only other procedure which is developed under within the frequency domain.

\subsection{2 $\mathrm{Q}_{n, m}(\mathrm{~s}, \mathrm{~d})$ Statistic}

In the forthcoming research of Ould-Haye \& Phillippe (2019), the authors developed a statistical procedure for testing stationarity with a certain dependence structure under the null against the alternate of the presence of a stochastic or deterministic 
trend. The proposed test statistic takes on the form of

$$
Q_{n, m}(s, d)=\left(m^{-2 d}\right) \sum_{j=1}^{s} \frac{I_{n}\left(\omega_{j}\right)}{\frac{1}{m} \sum_{k=1}^{m} I_{n, k}\left(\omega_{j}^{\prime}\right)}
$$

where $I_{n}\left(\omega_{j}\right)$ is the periodogram and $I_{n, k}\left(\omega_{j}\right)$ is the periodogram of the "kth" block. The window parameter $s$ is fixed and the test statistic depends on the Fourier frequencies, $\omega_{j}=\frac{2 \pi j}{n}$ and $\omega_{j}^{\prime}=\frac{2 \pi j}{l}$. Similar to Gromykov et al. (2018) the parameters $m$ and $l=\frac{n}{m}$ are functions of $n$ such that $m=m(n)$ and $l=l(n) \rightarrow \infty$ as $n \rightarrow \infty$.

The following theorem is taken from Ould-Haye \& Phillippe (2019) and provides the limiting distribution of the test statistic underneath the null and alternate hypothesis.

Theorem 3.2. (a) If $X_{t}$ is a linear process of the form of Proposition (2.3) admitting a spectral density of the form of (2.6) with $d \in(-.5, .5)$ and $Q_{n, m}(s, d)$ defined as in (3.18) where $s \geq 1$ is fixed. Letting $l=O\left(n^{1-\delta}\right)$ and $m=O\left(n^{1-\delta^{\prime}}\right)$ for some $\delta, \delta^{\prime}>0$, then

$$
Q_{n, m}(s, d) \stackrel{\mathbb{D}}{\longrightarrow} Q(s, d) .
$$

Where

$$
\begin{aligned}
& Q(s, d)=\sum_{j=1}^{n}\left(\frac{1}{2}\left(\left(Z_{1}^{U T}(j)\right)^{2}+\left(Z_{2}^{U T}(j)\right)^{2}\right)-\frac{R_{j}(d)}{L_{j}(d)}\left(\left(Z_{1}^{U T}(j)\right)^{2}-\left(Z_{2}^{U T}(j)\right)^{2}\right)\right) \\
& \text { Where }\left(Z_{1}^{U T}(1), Z_{2}^{U T}(1), \ldots, Z_{1}^{U T}(s), Z_{2}^{U T}(s)\right) \text { is a zero mean Gaussian vector, for }
\end{aligned}
$$


$j, k=1, \ldots, s$ with components given in (3.9). Moreover, $L_{j}(d)$ and $R_{j}(d)$ are given in (3.1) and (3.8), respectively.

(b) if $X_{t}=X_{t-1}+Y_{t}$ or if $X_{t}=g_{n}(t)+Y_{t}$ where $g_{n}(t)=n^{\alpha} g(t)$, where $\alpha>0$, $g$ is non-constant and square integratable on $(0,1)$, and $Y_{t}$ is a linear process with spectral density of the form of (2.6), then for any $0 \leq \eta \leq \frac{1}{2}$,

$$
Q_{n, m}(s, \eta) \stackrel{\mathbb{P}}{\longrightarrow} \infty .
$$

This testing procedure requires the memory parameter $d$ to be estimated. The following proposition depicts how the procedure is conducted within Ould-Haye \& Phillippe (2019). The authors show that if one chooses an estimator of $d$ that is consistent such that $\hat{d}-d=o_{p}(1 / \ln n)$ then results of the asymptotic distribution assumptions under the null and alternate hypothesis hold by replacing the true value of $d$ by this estimator. It should be noted that similar to Giraistis et al.(2005) the procedure penalizes $\hat{d}$ as bounds it between $[-.5, .5]$. Since our procedure will be built off of these results will elaborate more by providing Proposition 2 from Ould Haye \& Phillippe (2019) below.

Proposition 3.3. (a) If $X_{t}$ is a stationary linear process of the form of Proposition (2.3) with spectral density of the form of (2.6) and if $\hat{d}$ is a consistent estimator of $d$ such that $\hat{d}-d=o_{P}(1 / \ln n)$, then

$$
Q_{n, m}(s, \hat{d}) \stackrel{\mathbb{D}}{\longrightarrow} Q(s, d) .
$$

(b) if $X_{t}=X_{t-1}+Y_{t}$ or if $X_{t}=g_{n}(t)+Y_{t}$ where $g_{n}(t)=n^{\alpha} g(t)$, where $\alpha>0, g$ 
is square integratable on $(0,1)$, and $Y_{t}$ is a linear process with spectral density of the form of (2.6), and $\hat{d}$ is an esimtaor of $d$ based on $X_{1}, \ldots, X_{n}$ with $|\hat{d}| \leq 1 / 2$, then

$$
Q_{n, m}(s, \hat{d}) \stackrel{\mathbb{P}}{\longrightarrow} \infty .
$$

The authors go on to show from their simulation studies that the proposed test is robust in obtaining the correct theoretical size with both Gaussian and non-Gaussian innovations under the null hypothesis. As of this point we have reviewed and presented the concepts needed to more fully understand the derivations of our statistical tests. Therefore, we now proceed to the next section which states how these statistics are exactly developed.

\section{Methodology}

Within this section we derive all of the test statistics reported within our research. As one will notice the notation we have utilized is largely attributable to the notation implemented within Hurvich \& Beltrao (1993), Gromykov et al.(2018), and Ould Haye \& Phillippe (2019). This has been performed to allow for clarity when assessing our research alongside any of the previous authors. We also note some initial assumptions in regards to variables involved with our test statistics. The variable $n$ refers to the

sample length, $m=\frac{n}{l}$ refers the amount of blocks, and $l$ refers to the length of the block. Within our research the variables $m=m(n)$ and $l=l(n)$ are not fixed and depend on the sample size. We also note that the Fourier frequencies we implement 
within our research are $\omega_{j}=\frac{2 \pi j}{n}$ and $\omega_{j}^{\prime}=\frac{2 \pi j}{l}$, where $j$ indicates the "jth " Fourier frequency in which is being evaluated and which for this research we assume is fixed. We are primarily interested in the first few Fourier frequencies as they are known to contain the highest amount of statistical information in regards to the processes memory structure. Moreover, unless stated otherwise we assume that $j$ is an integer such that $j \in[1, M]$, where $M$ is a fixed integer as $n \rightarrow \infty$. Furthermore, assume that $m=o(n)$ and $l=o(n)$. We also note that the parameter $s$ refers to the amount of Fourier frequencies utilized within our test statistics and is assumed to be fixed. We now proceed to explain the derivation of the test statistics with the cosine bell taper within this next section.

\subsection{Cosine Bell Taper}

The first defintion we present is in regards to the explicit form of the cosine bell taper we implement within our research.

Definition 4.1. Let $t=1, \ldots, n$, where $n$ is the sample size. The following is known as asymmetric version of the cosine bell taper as Velasco (1999a) states and takes on the form

$$
h_{t}=\frac{1}{2}\left(1-\cos \left(\frac{2 \pi t}{n}\right)\right)=\sin ^{2}\left(\frac{\pi t}{n}\right) .
$$

We note that within this research $h_{t}$ solely refers to the cosine bell taper given in equation (4.1). However, at times we will interchange $n$ to $l$ within $h_{t}$ when applying 
the taper to the periodogram of the blocks. With the above it is useful to note that

$$
\sum_{t=1}^{n} h_{t}^{2}=\sum_{t=1}^{n} \sin ^{4}\left(\frac{\pi t}{n}\right)=\frac{1}{4} \sum_{t=1}^{n}\left(1-2 \cos \left(\frac{2 \pi t}{n}\right)+\frac{1}{2}\left(1-\cos \left(\frac{4 \pi t}{n}\right)\right)\right)=\frac{3 n}{8} .
$$

Similar to the derivation performed in Hurvich \& Beltrao (1993) we dissect the tapered periodogram to derive the limiting form of its moments. We define the discrete Fourier transform (DFT) of the tapered periodogram with the cosine bell taper to be

$$
D_{T}\left(\omega_{j}\right)=\frac{1}{\sqrt{2 \pi \sum_{t=1}^{n} h_{t}^{2}}} \sum_{t=1}^{n} h_{t} X_{t} e^{-i \omega_{j} t}=\frac{2}{\sqrt{3 \pi n}} \sum_{t=1}^{n} h_{t} X_{t} e^{-i \omega_{j} t}
$$

Furthermore, the tapered periodogram can be represented as:

$$
I_{n}^{T}\left(\omega_{j}\right)=\frac{4}{3 \pi n}\left|\sum_{t=1}^{n} h_{t} X_{t} e^{-i \omega_{j} t}\right|^{2}
$$

Dividing the sample of $n$ observations from the series $X_{t}$ into $m$ blocks of length $\frac{n}{m}=l$, We define the "kth" block's tapered periodogram to be

$$
I_{n, k}^{T}\left(\omega_{j}\right)=\frac{4}{3 \pi l} \sum_{t=1+(k-1) l}^{k l}\left|h_{t} X_{t} e^{-i \omega_{j} t}\right|^{2}
$$

It can be noted that

$$
I_{n}^{T}\left(\omega_{j}\right)=\left|D_{T}\left(\omega_{j}\right)\right|^{2}=\left|A_{T}\left(\omega_{j}\right)\right|^{2}+\left|B_{T}\left(\omega_{j}\right)\right|^{2}
$$

Where the tapered DFT can be broken down into the following forms

$$
D_{T}\left(\omega_{j}\right)=A_{T}\left(\omega_{j}\right)-i B_{T}\left(\omega_{j}\right)
$$

with

$$
A_{T}\left(\omega_{j}\right)=\frac{2}{\sqrt{3 \pi n}} \sum_{t=1}^{n} X_{t} \sin ^{2}\left(\frac{\pi}{n}\right) \cos \left(\omega_{j} t\right)
$$


and

$$
B_{T}\left(\omega_{j}\right)=\frac{2}{\sqrt{3 \pi n}} \sum_{t=1}^{n} X_{t} \sin ^{2}\left(\frac{\pi t}{n}\right) \sin \left(\omega_{j} t\right) .
$$

In order to further decompose the above, we define the following functions

$$
P_{n}(\theta, j)=\sum_{t=1}^{n} \sin ^{2}\left(\frac{\pi t}{n}\right) \cos \left(\omega_{j} t\right) e^{i t \theta}, \quad Q_{n}(\theta, j)=\sum_{t=1}^{n} \sin ^{2}\left(\frac{\pi t}{n}\right) \sin \left(\omega_{j} t\right) e^{i t \theta}
$$

Furthermore, with the functions directly above the components of the tapered DFT can represented in a more evaluative form assuming the process $X_{t}$ is a zero mean stationary process by using the Spectral Representation Theorem

$$
\begin{aligned}
A_{T}\left(\omega_{j}\right) & =\frac{2}{\sqrt{3 \pi n}} \sum_{t=1}^{n} X_{t} \sin ^{2}\left(\frac{\pi t}{n}\right) \cos \left(\omega_{j} t\right) \\
& =\frac{2}{\sqrt{3 \pi n}} \sum_{t=1}^{n} \int_{-\pi}^{\pi} \sin ^{2}\left(\frac{\pi t}{n}\right) \cos \left(\omega_{j} t\right) e^{i \theta t} d Z(\theta), \quad\left(\text { as } X_{t}=\int_{-\pi}^{\pi} e^{i \theta t} d Z(\theta)\right), \\
& =\frac{2}{\sqrt{3 \pi n}} \int_{-\pi}^{\pi} P_{n}(\theta, j) d Z(\theta) .
\end{aligned}
$$

Moreover,

$$
\begin{aligned}
B_{T}\left(\omega_{j}\right) & =\frac{2}{\sqrt{3 \pi n}} \sum_{t=1}^{n} X_{t} \sin ^{2}\left(\frac{\pi t}{n}\right) \sin \left(\omega_{j} t\right) \\
& =\frac{2}{\sqrt{3 \pi n}} \sum_{t=1}^{n} \int_{-\pi}^{\pi} \sin ^{2}\left(\frac{\pi t}{n}\right) \sin \left(\omega_{j} t\right) e^{i \theta t} d Z(\theta) \\
& =\frac{2}{\sqrt{3 \pi n}} \int_{-\pi}^{\pi} Q_{n}(\theta, j) d Z(\theta)
\end{aligned}
$$

With the above in mind, we can now proceed to deriving the asymptotic variance and covariance of the components of the tapered DFT.

Proposition 4.2. Assume that $X_{t}$ is of the form of Proposition (2.3) with spectral density $f$ of the form of (2.6) where $d \in(-.5, .5)$, then 
a.)

$$
\lim _{n \rightarrow \infty} \operatorname{Cov}\left(\frac{A_{T}\left(\omega_{j}\right)}{\sqrt{f\left(\omega_{j}\right)}}, \frac{A_{T}\left(\omega_{k}\right)}{\sqrt{f\left(\omega_{k}\right)}}\right)=\frac{16 \pi^{3}}{3} \int_{-\infty}^{\infty} \sin ^{2}\left(\frac{\theta}{2}\right) P(\theta, j) P(\theta, k)\left(\frac{\theta}{\sqrt{2 \pi j k}}\right)^{-2 d} d \theta
$$

b.)

$$
\lim _{n \rightarrow \infty} \operatorname{Cov}\left(\frac{B_{T}\left(\omega_{j}\right)}{\sqrt{f\left(\omega_{j}\right)}}, \frac{B_{T}\left(\omega_{k}\right)}{\sqrt{f\left(\omega_{k}\right)}}\right)=\frac{16 \pi^{3}}{3} \int_{-\infty}^{\infty} \sin ^{2}\left(\frac{\theta}{2}\right) Q(\theta, j) Q(\theta, k)\left(\frac{\theta}{2 \pi \sqrt{j k}}\right)^{-2 d} d \theta .
$$

c.)

$$
\lim _{n \rightarrow \infty} \operatorname{Cov}\left(\frac{A_{T}\left(\omega_{j}\right)}{\sqrt{f\left(\omega_{j}\right)}}, \frac{B_{T}\left(\omega_{k}\right)}{\sqrt{f\left(\omega_{k}\right)}}\right)=0
$$

Where $P(\theta, j)$ and $Q(\theta, j)$ are given in Proposition (9.8).

Proof. The proof for each part has been fully derived within Proposition (9.10).

With above theorem in mind we now state the asymptotic distribution for the tapered periodogram.

Theorem 4.3. Assume that $X_{t}$ is of the form of Proposition (2.3) with spectral density $f$ of the form of (2.6) where $d \in(-.5, .5)$, then

$$
\left(\frac{A_{T}\left(\omega_{1}\right)}{\sigma_{A_{1, n}} \sqrt{f\left(\omega_{1}\right)}}, . ., \frac{A_{T}\left(\omega_{s}\right)}{\sigma_{A_{s, n}} \sqrt{f\left(\omega_{s}\right)}}, \frac{B_{T}\left(\omega_{1}\right)}{\sigma_{B_{1, n}} \sqrt{f\left(\omega_{1}\right)}}, . ., \frac{B_{T}\left(\omega_{s}\right)}{\sigma_{B_{s, n}} \sqrt{f\left(\omega_{s}\right)}}\right) \stackrel{\mathbb{D}}{\longrightarrow} N_{2 s}\left(0, \Sigma_{2 s, 2 s}\right),
$$

where

$$
\Sigma_{2 s, 2 s}=\left[\begin{array}{cc}
A & 0_{s} \\
0_{s} & B
\end{array}\right] .
$$


With

$$
A=\left[\begin{array}{ccccc}
1 & a_{12} & a_{13} & \ldots & a_{1 s} \\
a_{21} & 1 & a_{23} & \ldots & a_{2 s} \\
\ldots & \ldots & \ldots & \ldots & \ldots \\
a_{s 1} & a_{s 2} & a_{s 3} & \ldots & 1
\end{array}\right], B=\left[\begin{array}{ccccc}
1 & b_{12} & b_{13} & \ldots & a_{1 s} \\
b_{21} & 1 & b_{23} & \ldots & a_{2 s} \\
\ldots \ldots & \ldots & \ldots & \ldots \\
\ldots & \ldots & \ldots & \ldots & \\
b_{n 1} & b_{s 2} & b_{s 3} & \ldots & 1
\end{array}\right]
$$

where $A$ and $B$ are the covariance matrices for the vectors $\left(\frac{A_{T}\left(\omega_{1}\right)}{\sigma_{A, 1, n} \sqrt{f\left(\omega_{1}\right)}}, \ldots, \frac{A_{T}\left(\omega_{s}\right)}{\sigma_{A, s, n} \sqrt{f\left(\omega_{s}\right)}}\right)$ and $\left(\frac{B_{T}\left(\omega_{1}\right)}{\sigma_{B, 1, n} \sqrt{f\left(\omega_{1}\right)}}, \ldots, \frac{B_{T}\left(\omega_{s}\right)}{\sigma_{B, s, n} \sqrt{f\left(\omega_{s}\right)}}\right)$, respectively. The matrix $0_{s}$ is the cross covariance of the vectors. The entries of the covariance matrices $A$ and $B$ are taken from the results of Proposition (4.2) and can be represented as

$$
a_{j, k}=\lim _{n \rightarrow \infty} \operatorname{Cov}\left(\frac{A_{T}\left(\omega_{j}\right)}{\sigma_{A, j, n} \sqrt{f\left(\omega_{j}\right)}}, \frac{A_{T}\left(\omega_{k}\right)}{\sigma_{A, k, n} \sqrt{f\left(\omega_{k}\right)}}\right),
$$

and

$$
b_{j, k}=\lim _{n \rightarrow \infty} \operatorname{Cov}\left(\frac{B_{T}\left(\omega_{j}\right)}{\sigma_{B, j, n} \sqrt{f\left(\omega_{j}\right)}}, \frac{B_{T}\left(\omega_{k}\right)}{\sigma_{B, k, n} \sqrt{f\left(\omega_{k}\right)}}\right)
$$

Where

$$
\begin{aligned}
& \sigma_{A, j, n}^{2}(d)=\operatorname{Var}\left(\frac{A_{T}\left(\omega_{j}\right)}{\sqrt{f\left(\omega_{j}\right)}}\right)=\operatorname{Cov}\left(\frac{A_{T}\left(\omega_{j}\right)}{\sqrt{f\left(\omega_{j}\right)}}, \frac{A_{T}\left(\omega_{j}\right)}{\sqrt{f\left(\omega_{j}\right)}}\right) . \\
& \sigma_{B, j, n}^{2}(d)=\operatorname{Var}\left(\frac{B_{T}\left(\omega_{j}\right)}{\sqrt{f\left(\omega_{j}\right)}}\right)=\operatorname{Cov}\left(\frac{B_{T}\left(\omega_{j}\right)}{\sqrt{f\left(\omega_{j}\right)}}, \frac{B_{T}\left(\omega_{j}\right)}{\sqrt{f\left(\omega_{j}\right)}}\right),
\end{aligned}
$$

and

$$
\lim _{n \rightarrow \infty} \sigma_{A, j, n}^{2}(d)=\sigma_{A, j}^{2}(d) \quad \quad \lim _{n \rightarrow \infty} \sigma_{B, j, n}^{2}(d)=\sigma_{B, j}^{2}(d) .
$$

Moreover, we also note that for simplicity in regards to our notation we denote $\sigma_{A, j, n}^{2}(d)=\sigma_{A, j, n}^{2}$ and $\sigma_{B, j, n}^{2}(d)=\sigma_{B, j, n}^{2}$ 
Proof. By Theorem (9.17) we can see that for $d \in(-.5, .5)$

$$
\max _{1 \leq t \leq n}\left|z_{n t, A, j}\right|=o\left(\sigma_{A, j . n}\right), \quad \quad \max _{1 \leq t \leq n}\left|z_{n t, B, j}\right|=o\left(\sigma_{B, j, n}\right)
$$

For $d \in(0, .5)$ by Theorem (9.18) We have that

$$
\sum_{t=1}^{n} z_{n t, A, j}^{2}=O\left(\sigma_{A, j, n}^{2}\right), \quad \quad \sum_{t=1}^{n} z_{n t, B, j}^{2}=O\left(\sigma_{B, j, n}^{2}\right)
$$

Hence condition (i) of Theorem (2.5) is satisfied. In the case of $d \in(-.5, .0]$ we have the moving average coefficients of $X_{t}$ from Proposition (2.3) satisfying

$$
\sum_{k=1}^{\infty}\left|\psi_{k}\right|<\infty
$$

Therefore, condition (ii) of Theorem (2.5) is satisfied and hence, by Theorem (2.5) we have

$$
\left(\frac{A_{T}\left(\omega_{1}\right)}{\sigma_{A, 1, n} \sqrt{f\left(\omega_{1}\right)}}, . ., \frac{A_{T}\left(\omega_{s}\right)}{\sigma_{A, s, n} \sqrt{f\left(\omega_{s}\right)}}, \frac{B_{T}\left(\omega_{1}\right)}{\sigma_{B, 1, n} \sqrt{f\left(\omega_{1}\right)}}, . ., \frac{B_{T}\left(\omega_{s}\right)}{\sigma_{B, s, n} \sqrt{f\left(\omega_{s}\right)}}\right) \stackrel{\mathbb{D}}{\longrightarrow} \mathcal{Z}^{T}
$$

where $\mathcal{Z}^{T}$ is a $N_{2 s}\left(0, \Sigma_{2 s, 2 s, d}\right)$ random vector of standard Gaussian components $Z_{1}(j), Z_{2}(j)$ that takes on the form of

$$
\mathcal{Z}^{T}=\left(Z_{1}(1), \ldots, Z_{1}(s), Z_{2}(1), \ldots, Z_{2}(s)\right)
$$

We note that the covariance matrix is a function of the memory parameter $d$. We note that the cosine and sine components are asymptotically independent of one another since they are jointly normally distributed and the cross covariances asymptotically go to zero which we depict in Lemma (9.16) which states

$$
\lim _{n \rightarrow \infty} \operatorname{Cov}\left(\frac{A_{T}\left(\omega_{j}\right)}{\sigma_{A, j, n} \sqrt{f\left(\omega_{j}\right)}}, \frac{B_{T}\left(\omega_{k}\right)}{\sigma_{B, k, n} \sqrt{f\left(\omega_{k}\right)}}\right)=0
$$


Therefore, $0_{s}$ represents the cross covariance matrix between $A_{T}$ and $B_{T}$.

With the above in mind, we note that

$$
P(\theta, j)=G(\theta+2 \pi j)+G(\theta-2 \pi j), \quad Q(\theta, j)=G(\theta-2 \pi j)-G(\theta+2 \pi j),
$$

where

$$
G(\theta)=\frac{1}{\theta(\theta+2 \pi)(\theta-2 \pi)} .
$$

Moreover, using the notation from Hurvich \& Beltrao (1993) we define

$L_{j, T}(d)=\sigma_{A, j}^{2}+\sigma_{B, j}^{2}=\frac{32 \pi^{3}}{3} \int_{-\infty}^{\infty} \sin ^{2}\left(\frac{\theta}{2}\right)\left((G(\theta+2 \pi j))^{2}+(G(\theta-2 \pi j))^{2}\right)\left|\frac{\theta}{2 \pi j}\right|^{-2 d} d \theta$,

and

$$
R_{j, T}(d)=\frac{32 \pi^{3}}{3} \int_{-\infty}^{\infty} \sin ^{2}\left(\frac{\theta}{2}\right) G(\theta+2 \pi j) G(\theta-2 \pi j)\left|\frac{\theta}{2 \pi j}\right|^{-2 d} d \theta
$$

Therefore,

$$
\sigma_{A, j}^{2}=\frac{1}{2} L_{j, T}(d)+R_{j, T}(d), \quad \sigma_{B, j}^{2}=\frac{1}{2} L_{j, T}(d)-R_{j, T}(d),
$$

since

$$
\begin{aligned}
& \sigma_{A, j}^{2}=\lim _{n \rightarrow \infty} \operatorname{Var}\left(\frac{A_{T}\left(\omega_{j}\right)}{\sqrt{f\left(\omega_{j}\right)}}\right)=\frac{16 \pi^{3}}{3} \int_{-\infty}^{\infty} \sin ^{2}\left(\frac{\theta}{2}\right) P(\theta, j)^{2}\left|\frac{\theta}{2 \pi j}\right|^{-2 d} d \theta \\
& =\frac{16 \pi^{3}}{3} \int_{-\infty}^{\infty} \sin ^{2}\left(\frac{\theta}{2}\right)(G(\theta+2 \pi j)+G(\theta-2 \pi j))^{2}\left|\frac{\theta}{2 \pi j}\right|^{-2 d} d \theta \\
& \left.=\frac{16 \pi^{3}}{3} \int_{-\infty}^{\infty} \sin ^{2}\left(\frac{\theta}{2}\right)\left((G(\theta+2 \pi j))^{2}+2 G(\theta+2 \pi j) G(\theta-2 \pi j)\right)+(G(\theta-2 \pi j))^{2}\right)\left|\frac{\theta}{2 \pi j}\right|^{-2 d} d \theta \\
& =\frac{1}{2} L_{j, T}(d)+R_{j, T}(d),
\end{aligned}
$$


and

$$
\begin{aligned}
& \sigma_{B, j}^{2}=\lim _{n \rightarrow \infty} \operatorname{Var}\left(\frac{B_{T}\left(\omega_{j}\right)}{\sqrt{f\left(\omega_{j}\right)}}\right)=\frac{16 \pi^{3}}{3} \int_{-\infty}^{\infty} \sin ^{2}\left(\frac{\theta}{2}\right) Q(\theta, j)^{2}\left|\frac{\theta}{2 \pi j}\right|^{-2 d} d \theta \\
& =\frac{16 \pi^{3}}{3} \int_{-\infty}^{\infty} \sin ^{2}\left(\frac{\theta}{2}\right)(G(\theta+2 \pi j)-G(\theta-2 \pi j))^{2}\left|\frac{\theta}{2 \pi j}\right|^{-2 d} d \theta \\
& \left.=\frac{16 \pi^{3}}{3} \int_{-\infty}^{\infty} \sin ^{2}\left(\frac{\theta}{2}\right)\left((G(\theta+2 \pi j))^{2}-2 G(\theta+2 \pi j) G(\theta-2 \pi j)\right)+(G(\theta-2 \pi j))^{2}\right)\left|\frac{\theta}{2 \pi j}\right|^{-2 d} d \theta \\
& =\frac{1}{2} L_{j, T}(d)-R_{j, T}(d) .
\end{aligned}
$$

Definition 4.4. We define the random variables $Z_{1}(j)$ and $Z_{2}(j)$ in which the components of the tapered periodogram converge in distribution to in Theorem (4.3). We note that the notation for $Z_{1}(j)$ and $Z_{2}(j)$ is saved exclusively for these random variables. We also note that $Z_{1}(j)$ and $Z_{2}(j)$ have the following property

$$
\operatorname{Cov}\left(Z_{1}(j), Z_{2}(k)\right)=0, \quad \forall j, k
$$

With the above in mind we now derive the limiting bias of the normalized tapered periodogram.

Proposition 4.5. Assume that $X_{t}$ is of the form of Proposition (2.3) with spectral density $f$ of the form of (2.6) where $d \in(-.5, .5)$, then

$$
\lim _{n \rightarrow \infty} \mathbb{E}\left(\frac{I_{n}^{T}\left(\omega_{j}\right)}{f\left(\omega_{j}\right)}\right)=L_{j, T}(d), \quad \quad \lim _{n \rightarrow \infty} \mathbb{E}\left(\frac{I_{n, k}^{T}\left(\omega_{j}^{\prime}\right)}{f\left(\omega_{j}^{\prime}\right)}\right)=L_{j, T}(d)
$$

Proof. It can be demonstrated that

$$
\lim _{n \rightarrow \infty} \mathbb{E}\left(\frac{I_{n}^{T}\left(\omega_{j}\right)}{f\left(\omega_{j}\right)}\right)=\lim _{n \rightarrow \infty} \mathbb{E}\left(\left|\frac{A_{T}\left(\omega_{j}\right)}{\sqrt{f\left(\omega_{j}\right)}}\right|^{2}\right)+\lim _{n \rightarrow \infty} \mathbb{E}\left(\left|\frac{B_{T}\left(\omega_{j}\right)}{\sqrt{f\left(\omega_{j}\right)}}\right|^{2}\right)=\sigma_{A, j}^{2}+\sigma_{B, j}^{2}=L_{j, T}(d) .
$$


A similar proof can be performed for the periodogram of the "kth" block as

$$
h_{t}=\sin ^{2}\left(\frac{\pi t}{l}\right)=\sin ^{2}\left(\frac{(\pi t+\pi(1-k) l)}{l}\right)=h_{t+(1-k) l} .
$$

Therefore,

$$
\mathbb{E}\left(h_{t} h_{s} X_{t} X_{s}\right)=\mathbb{E}\left(h_{t+(1-k) l} h_{s+(1-k) l} X_{t+(1-k) l} X_{s+(1-k) l}\right)=h_{t} h_{s} \gamma(t-s) .
$$

We now present concepts which allow us to further derive our test statistics.

Proposition 4.6. Let $X_{t}$ be of the form of Proposition (2.3) with spectral density $f$ of the form of (2.6) where $d \in(-.5, .5)$, then

$$
\frac{1}{m} \sum_{k=1}^{m} \frac{I_{n, k}^{T}\left(\omega_{j}^{\prime}\right)}{f\left(\omega_{j}^{\prime}\right)} \stackrel{\mathbb{P}}{\longrightarrow} L_{j, T}(d), \quad \frac{1}{m} \sum_{k=1}^{m} \frac{I_{n, k}^{T}\left(\omega_{j}\right)}{f\left(\omega_{j}\right)} \stackrel{\mathbb{P}}{\longrightarrow} L_{j, T}(d) .
$$

Proof. The proof which is similar to Ould Haye \& Phillippe (2019) can be found within the appendix in Proposition (9.13).

With the above concepts now well defined we will now proceed to derive the asymptotic distribution of certain functional forms involving normalized tapered periodogram and the averaged tapered periodogram in the following proposition.

Proposition 4.7. Let $X_{t}$ be of the form of Proposition (2.3) with spectral density $f$ of the form of (2.6) where $d \in(-.5, .5)$, then

a.)

$$
\left(\frac{I_{n}^{T}\left(\omega_{1}\right)}{f\left(\omega_{1}\right)}, . ., \frac{I_{n}^{T}\left(\omega_{s}\right)}{f\left(\omega_{s}\right)}\right) \stackrel{\mathbb{D}}{\longrightarrow}\left(W_{1}, \ldots, W_{s}\right)
$$


Where

$$
\begin{aligned}
& W_{j}=\sigma_{A, j}^{2} Z_{1}^{2}(j)+\sigma_{B, j}^{2} Z_{s}^{2}(j)=\frac{L_{j, T}(d)}{2}\left(Z_{1}^{2}(j)+Z_{2}^{2}(j)\right)+R_{j, T}(d)\left(Z_{1}^{2}(j)-Z_{2}^{2}(j)\right) . \\
& \text { b.) } \\
& \sum_{j=1}^{s} \frac{I_{n}^{T}\left(\omega_{j}\right) / f\left(\omega_{j}\right)}{\frac{1}{m} \sum_{k=1}^{m} I_{n, k}\left(\omega_{j}^{\prime}\right) / f\left(\omega_{j}^{\prime}\right)} \stackrel{\mathbb{D}}{\longrightarrow} \sum_{j=1}^{s}\left(\frac{1}{2}\left(Z_{1}^{2}(j)+Z_{2}^{2}(j)\right)+\frac{R_{j, T}(d)}{L_{j, T}(d)}\left(Z_{1}^{2}(j)-Z_{2}^{2}(j)\right)\right) .
\end{aligned}
$$

Proof. Part a.) Using the asymptotic joint distribution of the $A_{T}, B_{T}$ components from Theorem (4.3) in conjunction with the Continuous Mapping Theorem yields

$$
\left(\frac{A_{T}\left(\omega_{1}\right)}{\sqrt{f\left(\omega_{1}\right)}}, . ., \frac{A_{T}\left(\omega_{s}\right)}{\sqrt{f\left(\omega_{s}\right)}}, \frac{B_{T}\left(\omega_{1}\right)}{\sqrt{f\left(\omega_{1}\right)}}, . ., \frac{B_{T}\left(\omega_{s}\right)}{\sqrt{f\left(\omega_{s}\right)}}\right) \stackrel{\mathbb{D}}{\longrightarrow} Z^{T}
$$

where

$$
Z^{T}=\left(\sigma_{A, 1} Z_{1}(1), \ldots, \sigma_{A, s} Z_{1}(s), \sigma_{B, 1} Z_{2}(1), \ldots, \sigma_{B, 2} Z_{2}(s)\right)
$$

By applying the Continuous Mapping Theorem once again to the above it can be seen individually for each $j$

$$
\frac{I_{n}^{T}\left(\omega_{j}\right)}{f\left(\omega_{j}\right)}=\left|\frac{A_{T}\left(\omega_{j}\right)}{\sqrt{f\left(\omega_{j}\right)}}-i \frac{B_{T}\left(\omega_{j}\right)}{\sqrt{f\left(\omega_{j}\right)}}\right|^{2}=\left|\frac{A_{T}\left(\omega_{j}\right)}{\sqrt{f\left(\omega_{j}\right)}}\right|^{2}+\left|\frac{B_{T}\left(\omega_{j}\right)}{\sqrt{f\left(\omega_{j}\right)}}\right|^{2} \stackrel{\mathbb{D}}{\longrightarrow} W_{j} .
$$

Therefore, applying the Continuous Mapping Theorem to (4.4) for each $j$ we have $(4.3)$.

Part b.) From Proposition (4.6) we have the normalized tapered periodogram averaged over the $m$ blocks converging in probability to a non-random limit as

$$
\frac{1}{m} \sum_{k=1}^{m} \frac{I_{n, k}^{T}\left(\omega_{j}^{\prime}\right)}{f\left(\omega_{j}^{\prime}\right)} \stackrel{\mathbb{P}}{\longrightarrow} L_{j, T}(d)
$$


Hence, utilizing Slutsky's Theorem with the results in (4.3) in conjunction with those from Proposition (4.6) yields

$$
\left(\frac{I_{n}^{T}\left(\omega_{1}\right) / f\left(\omega_{1}\right)}{\frac{1}{m} \sum_{k=1}^{m} I_{n, k}\left(\omega_{1}^{\prime}\right) / f\left(\omega_{1}^{\prime}\right)}, . ., \frac{I_{n}^{T}\left(\omega_{s}\right) / f\left(\omega_{s}\right)}{\frac{1}{m} \sum_{k=1}^{m} I_{n, k}\left(\omega_{s}^{\prime}\right) / f\left(\omega_{s}^{\prime}\right)}\right) \stackrel{\mathbb{D}}{\longrightarrow}\left(\frac{W_{1}}{L_{1, T}(d)}, \ldots, \frac{W_{s}}{L_{s, T}(d)}\right)
$$

Therefore, using the Continuous Mapping Theorem with the above gives us

$$
\sum_{j=1}^{s} \frac{I_{n}^{T}\left(\omega_{j}\right) / f\left(\omega_{j}\right)}{\frac{1}{m} \sum_{k=1}^{m} I_{n, k}\left(\omega_{j}^{\prime}\right) / f\left(\omega_{j}^{\prime}\right)} \stackrel{\mathbb{D}}{\longrightarrow} \sum_{j=1}^{s}\left(\frac{1}{2}\left(Z_{1}^{2}(j)+Z_{2}^{2}(j)\right)+\frac{R_{j, T}(d)}{L_{j, T}(d)}\left(Z_{1}^{2}(j)-Z_{2}^{2}(j)\right)\right)
$$

\subsubsection{Asymptotic Distribution Within the First Procedure}

With the above in mind we now derive the asymptotic distribution of the first proce-

dures test statistic using the cosine bell taper. This statistic we denote as $Q_{n, m}^{T}(s)$. We note that the null and alternate hypothesis are analogous to those conducted within Gromykov et al.(2018). Furthermore, we present this next theorem to allow for us to demonstrate the theoretical content in respect to the asymptotic distributions of this test.

Theorem 4.8. Assume that $X_{t}$ is of the form given in Proposition (2.3) with spectral density $f$ of the form of (2.6) where $d \in[0, .5)$.

We define the test statistic as

$$
Q_{n, m}^{T}(s)=\sum_{j=1}^{s} \frac{I_{n}^{T}\left(\omega_{j}\right)}{\frac{1}{m} \sum_{k=1}^{m} I_{n, k}^{T}\left(\omega_{j}^{\prime}\right)} .
$$


a.) If $X_{t}$ is a short memory process in a sense that $d=0$ and

$$
\sum_{k=1}^{\infty}\left|\psi_{k}\right|<\infty
$$

then

$$
Q_{n, m}^{T}(s) \stackrel{\mathbb{D}}{\rightarrow} Q^{T}(s)
$$

where

$$
Q^{T}(s)=\frac{1}{12}\left(Z_{1}^{2}(1)-Z_{2}^{2}(1)\right)+\frac{1}{2} \sum_{j=1}^{s}\left(Z_{1}^{2}(j)+Z_{2}^{2}(j)\right) .
$$

b.) If $X_{t}$ is a long memory process in the sense that $d \in(0, .5)$, then

$$
Q_{n, m}^{T}(s) \stackrel{\mathbb{P}}{\longrightarrow} \infty
$$

Proof. For both parts we note that since $f$ is of semi-parametric form we have $\forall d \in$ $[0, .5)$ the following when $n \rightarrow \infty$

$$
f\left(\omega_{j}^{\prime}\right) \sim C_{f}\left(\frac{l}{2 \pi j}\right)^{-2 d}, \quad f\left(\omega_{j}\right) \sim C_{f}\left(\frac{n}{2 \pi j}\right)^{-2 d},
$$

and therefore,

$$
\frac{f\left(\omega_{j}\right)}{f\left(\omega_{j}^{\prime}\right)} \sim\left(\frac{l}{n}\right)^{-2 d}=m^{2 d}
$$

Moreover by Part b.) in Proposition (4.7)

$$
\sum_{j=1}^{s} \frac{I_{n}^{T}\left(\omega_{j}\right) / f\left(\omega_{j}\right)}{\frac{1}{m} \sum_{k=1}^{m} I_{n, k}^{T}\left(\omega_{j}^{\prime}\right) / f\left(\omega_{j}^{\prime}\right)}=O_{P}(1)
$$

Hence,

$$
Q_{n, m}^{T}(s)=\sum_{j=1}^{s} \frac{I_{n}^{T}\left(\omega_{j}\right)}{\frac{1}{m} \sum_{k=1}^{m} I_{n, k}^{T}\left(\omega_{j}^{\prime}\right)}=\frac{f\left(\omega_{j}\right)}{f\left(\omega_{j}^{\prime}\right)} \sum_{j=1}^{s} \frac{I_{n}^{T}\left(\omega_{j}\right) / f\left(\omega_{j}\right)}{\frac{1}{m} \sum_{k=1}^{m} I_{n, k}^{T}\left(\omega_{j}^{\prime}\right) / f\left(\omega_{j}^{\prime}\right)}=O_{P}\left(m^{2 d}\right) .
$$


Part a.) Under short memory $d=0$ and therefore, from the above

$$
Q_{n, m}^{T}(s)=O_{P}(1)
$$

Using Slutsky's Theorem with Proposition (4.7) we have

$$
Q_{n, m}^{T}(s) \stackrel{\mathbb{D}}{\longrightarrow} Q^{T}(s),
$$

as $d=0, L_{j, T}(0)=1, R_{1, T}(0)=1 / 12, R_{j, T}(0)=0$ for $j \geq 2$.

Part b.) From the above $Q_{n, m}^{T}(s)=O_{p}\left(m^{2 d}\right)$. Therefore,

$$
Q_{n, m}^{T}(s) \stackrel{\mathbb{P}}{\longrightarrow} \infty
$$

Furthermore, for the $Q_{n, m}^{T}(s)$ test, letting $Q_{\alpha}^{T}(s)$ be the $1-\alpha$ quantile for the random variable $Q^{T}(s)$ we have the critical region being $\left\{Q_{n, m}^{T}(s)>Q_{\alpha}^{T}(s)\right\}$ which can be used to distinguish between short and long memory for a series that is assumed to satisfy the assumptions given in the above theorem. We note that this test is correct as given the results in Part a.) of the above we have

$$
P\left(Q_{n, m}^{T}(s)>Q_{\alpha}^{T}(s) \mid H_{0}\right) \rightarrow \alpha
$$

Moreover, given the results of the above in Part b.), in the case of a long memory process the testing procedure is consistent as $Q_{n, m}^{T}(s) \stackrel{\mathbb{P}}{\longrightarrow} \infty$ and therefore,

$$
P\left(\left|Q_{n, m}^{T}(s)\right|>c\right) \rightarrow 1, \text { as } c \rightarrow \infty .
$$


Hence,

$$
P\left(Q_{n, m}^{T}(s)>Q_{\alpha}^{T}(s) \mid H_{1}\right) \rightarrow 1
$$

With the first procedure described we now proceed to state the asymptotic distribution of our proposed test statistic with the cosine bell taper for the second procedure similar to the working paper of Ould Haye \& Phillippe (2019).

\subsubsection{Asymptotic Distribution Within the Second Procedure}

For the second procedure involving the cosine bell taper we note that the null and alternative hypotheses are largely inspired by Ould Haye \& Phillippe (2019). The following theorem now further ellaborates on our test.

Theorem 4.9. Assume that $l=O\left(n^{1-\delta}\right)$ with $m=O\left(n^{1-\delta^{\prime}}\right)$ for some $\delta, \delta^{\prime}>0$. Furthermore, let the test statistic be defined as

$$
Q_{n, m}^{T^{\prime}}(s, d)=m^{-2 d} \sum_{j=2}^{s} \frac{I_{n}^{T}\left(\omega_{j}\right)}{\frac{1}{m} \sum_{k=1}^{m} I_{n, k}^{T}\left(\omega_{j}^{\prime}\right)}
$$

a.) Let $X_{t}$ satisfy the form of Proposition (2.3) admitting a spectral density $f$ of the form of (2.6) where $d \in(-.5, .5)$, then

$$
\begin{aligned}
& Q_{n, m}^{T^{\prime}}(s, d) \stackrel{\mathbb{D}}{\longrightarrow} Q^{T^{\prime}}(s, d)=\sum_{j=2}^{s}\left(\frac{1}{2}\left(Z_{1}^{2}(j)+Z_{2}^{2}(j)\right)+\frac{R_{j, T}(d)}{L_{j, T}(d)}\left(Z_{1}^{2}(j)-Z_{2}^{2}(j)\right)\right) \\
& \text { b.) If } X_{t}=X_{t-1}+Y_{t} \text { or } X_{t}=g_{n}(t)+Y_{t} \text { where } g_{n}(t)=n^{\alpha} g(t) \text {, where } \alpha>0, g
\end{aligned}
$$

is non-constant and square integratable on $[0,1]$ and $Y_{t}$ is a linear process satisfying Proposition (2.3) with a spectral density of the form (2.6) where $d \in(-.5, .5)$, then 
for any $0 \leq \varsigma \leq 1 / 2$

$$
Q_{n, m}^{T^{\prime}}(s, \varsigma) \stackrel{\mathbb{P}}{\longrightarrow} \infty
$$

Proof. Noting once again that $\frac{f\left(\omega_{j}\right)}{f\left(\omega_{j}^{\prime}\right)} \sim\left(\frac{n}{l}\right)^{2 d}=m^{2 d}$, and

$$
Q_{n, m}^{T^{\prime}}(s, d)=m^{-2 d} \sum_{j=2}^{s} \frac{f\left(\omega_{j}\right)}{f\left(\omega_{j}^{\prime}\right)} \frac{I_{n}^{T}\left(\omega_{j}\right) / f\left(\omega_{j}\right)}{\frac{1}{m} \sum_{k=1}^{m} I_{n, k}\left(\omega_{j}^{\prime}\right) / f\left(\omega_{j}^{\prime}\right)} \sim \sum_{j=2}^{s} \frac{I_{n}^{T}\left(\omega_{j}\right) / f\left(\omega_{j}\right)}{\frac{1}{m} \sum_{k=1}^{m} I_{n, k}\left(\omega_{j}^{\prime}\right) / f\left(\omega_{j}^{\prime}\right)} .
$$

By Part b.) of Proposition (4.7) we have

$$
Q_{n, m}^{T^{\prime}}(s, d) \sim \sum_{j=2}^{s} \frac{I_{n}^{T}\left(\omega_{j}\right) / f\left(\omega_{j}\right)}{\frac{1}{m} \sum_{k=1}^{m} I_{n, k}\left(\omega_{j}^{\prime}\right) / f\left(\omega_{j}^{\prime}\right)} \stackrel{\mathbb{D}}{\longrightarrow} Q^{T^{\prime}}(s, d)
$$

where

$$
Q^{T^{\prime}}(s, d)=\sum_{j=2}^{s}\left(\frac{1}{2}\left(Z_{1}^{2}(j)+Z_{2}^{2}(j)\right)+\frac{R_{j, T}(d)}{L_{j, T}(d)}\left(Z_{1}^{2}(j)-Z_{2}^{2}(j)\right)\right)
$$

Part b.) The required results are proven within Proposition (9.15) and Proposition (9.14) which are located within the Appendix.

According to Part a.) of the above theorem the critical region of the procedure for testing stationarity versus the presence of a deterministic or stochastic trend at significance level $\alpha$ is of the form $\left\{Q_{n, m}^{T^{\prime}}(s, d)>Q_{\alpha}^{T^{\prime}}(s, d)\right\}$. Where $Q_{\alpha}^{T^{\prime}}(s, d)$ is the quantile of order $1-\alpha$ of the $Q^{T^{\prime}}(s, d)$ statistic. We note that the test is asymptotically correct given the results of Part a.).

Moreover, under the conditions of Part b.) of the above theorem, the test is consistent with any memory parameter $d$ and any normalizing coefficient $\varsigma \in[0, .5]$. 
As stated previously, in practice $d$ is unknown and therefore, will need to be estimated. We note that under the null hypothesis, the estimators $d_{G P H}, d_{G P H}^{T}$, and $d_{G P H}^{T^{\prime}}$ are all consistent for estimating $d$ such that $\hat{d}-d=o_{P}(1 / \ln n)$ as Velasco (1999b) states. Therefore, under the null conditions we can apply Slutsky's Theorem from the results of the above theorem to demonstrate for the testing procedure

$$
\frac{Q_{n, m}^{T^{\prime}}(s, \hat{d})}{Q_{n, m}^{T^{\prime}}(s, d)} \stackrel{\mathbb{P}}{\longrightarrow} 1 .
$$

Hence,

$$
P\left(Q_{n, m}^{T^{\prime}}(s, \hat{d})>Q_{\alpha}^{T^{\prime}}(s, \hat{d}) \mid H_{0}\right) \rightarrow \alpha,
$$

where $Q_{\alpha}^{T^{\prime}}(s, \hat{d})$ is the quantile of order $1-\alpha$ of $Q^{T^{\prime}}(s, \hat{d})$. Furthermore, utilizing an estimator $\hat{d}$ of $d$ with $|\hat{d}| \leq .5$ will generate poor results and will therefore tend to go outside $[-.5, .5]$ but will be penalized to $|\hat{d}| \leq .5$. So by taking $\varsigma=.5$ we will get

$$
Q_{n, m}(s, \hat{d}) \stackrel{\mathbb{P}}{\longrightarrow} \infty .
$$

Hence,

$$
P\left(Q_{n, m}^{T^{\prime}}(s, \hat{d})>Q_{\alpha}^{T^{\prime}}(s, \hat{d}) \mid H_{1}\right) \rightarrow 1 .
$$

We now speak of the random tapers we implement within this thesis.

\subsection{Random Tapering}

The results of Csorgo et al.(2017) alluded to the potential of finding improvements of the asymptotic properties of conventional estimators such as the ACVF and periodogram by implementing random weights. For instance, the authors showed that 
by applying a random weight to the conventional t-statistic and updating the pivot accordingly, the rate at which the pivot converged in distribution to that of a standard normal random variable improved underneath long memory in comparision to its classical counterpart. The authors also found that the coverage probability increased for the sample mean with the random weights in the case of short and long memory. These notable results inspired us to develop a random taper denoted by $a_{t}$ in which would be applied to the series $X_{t}$ in hopes of improving the asymptotic performance of the sample ACVF and periodogram in a manner that improves the estimation the ACVF and spectral density. Furthermore we placed the random tapers into practice by simulating from different process and estimating the ACVF with and without the taper. Some of the results from these can be seen from the results depicted in Figure (2) that random tapering reduces the covariance structure of the process. The figure has been generated from simulating with $\mathrm{AR}(1)$ samples of the form of $X_{t}=\phi X_{t-1}+\epsilon_{t}$, where $\phi$ takes on the values of $-.95, .95$ and $N(0,1)$ innovations. With these conditions we find that on average the sample ACF produced by the randomly tapered series is less that of the untapered series. When simulating from a long memory series similar results were found as the sample ACF decreased to a certain extent. From these simulations we speculate that perhaps the benefit of random tapering is the centering of the data in the case of non-Gaussian innovations for instance random tapering could tend to accelerate the convergence by generating a new process that is centered. Overall, the partial reduction in dependence makes 
random tapering appealing as many of the procedures included within our literature review section suffered from high empirical size when the dependence within the data was strong underneath certain null conditions. Another beneficial aspect of applying random tapering of the specified form given above, is that for the first procedure the limiting distributions are not altered from Gromykov et al.(2018) and therefore, is a simple Gamma like distribution under the null.

We have chosen to devise a test utilizing the random tapering for only the first procedure as performing random tapering in the case of negative memory alters the asymptotic rate of the first moment of the periodogram which induces undesirable results when attempting to create the test statistic for the second procedure. Moreover, for this taper to be implemented into the first procedure, certain assumptions regarding the behaviour of the taper need to be made. The following provides these.

Definition 4.10. Let $a_{t}$ be a random sample of a continuous random variable such that $\mathbb{E}\left(a_{t}\right)=\mu_{a} \neq 0$ and $\mathbb{E}\left(a_{t}^{2}\right)<\infty$. Let $a_{t}$ be independent of the process $\left(X_{t}\right)$ in which it is applied to. Moreover, assume $a_{t}$ is non-negative and uniformly bounded $\forall t$, then we refer to $\left\{a_{t} ; t=1, \ldots n\right\}$ as a random taper.

For simplicity, we have chosen one taper to measure the performance and the efficacy of random tapering. A taper that satisfies criteria given above is one from a uniform distribution on $[0,1]$. Hence, with the taper we implement each individual observation satisfies the following

$$
a_{t} \stackrel{i . i . d}{\sim} U(0,1)
$$



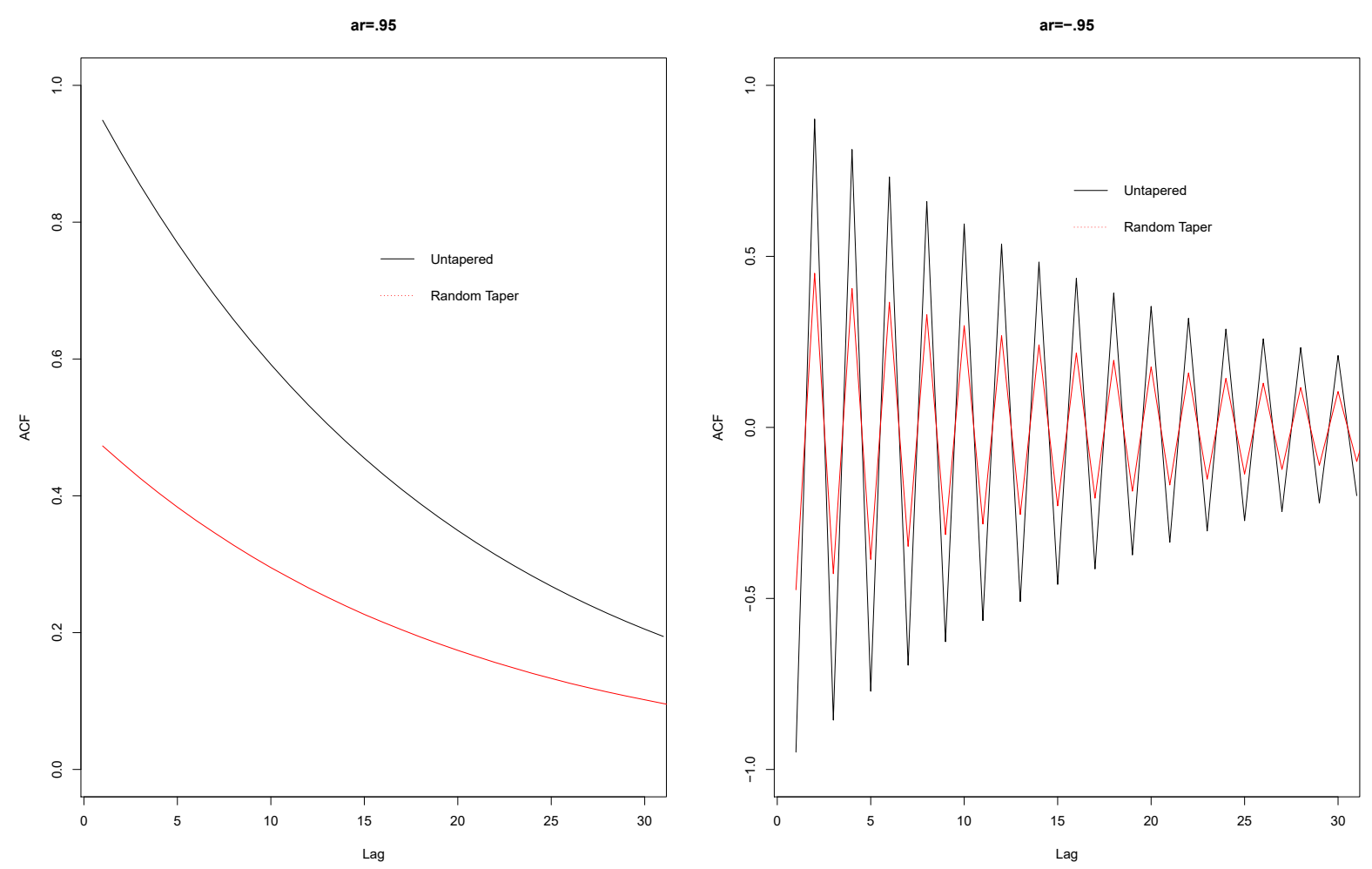

Figure 2: Untapered and Random Tapered ACF Simulations

The above graphs are the averaged ACF composed with and without random tapering within 5000 independent replications of sample size $n=1000$ from an AR(1) process with $N(0,1)$ innovations. The graph on the left and the right are composed with the the AR coefficient equaling .95 and -.95, respectively. 
It is useful to note that weights that satisfy (4.10) do not alter the dependence structure or the stationarity structure of a process satisfying the form of Proposition (2.3) for $d \in[0, .5)$. For instance, the process $a_{t} X_{t}$ is still stationary given that

$$
\begin{aligned}
& \mathbb{E}\left(a_{t} X_{t}\right)=\mathbb{E}\left(a_{t}\right) \mathbb{E}\left(X_{t}\right)=0, \\
& \operatorname{Var}\left(a_{t} X_{t}\right)=\mathbb{E}\left(a_{t}^{2} X_{t}^{2}\right)=\mathbb{E}\left(a_{t}^{2}\right) \mathbb{E}\left(X_{t}^{2}\right)=\mathbb{E}\left(a_{1}^{2}\right) \gamma_{X}(0)<\infty, \\
& \left.\operatorname{Cov}\left(a_{t} X_{t}, a_{s} X_{s}\right)=\mathbb{E}\left(a_{t} X_{t} a_{s} X_{s}\right)=\mathbb{E}\left(a_{t} a_{s}\right) \gamma_{X}(t-s)=\left(\mathbb{E}\left(a_{1}\right)\right)^{2} \gamma_{X}(h)\right), \forall t \neq s .
\end{aligned}
$$

The behaviour of the ACVF of the process $a_{t} X_{t}$ as $h \rightarrow \infty$ can be modelled as

$$
\left.\operatorname{Cov}\left(a_{t} X_{t}, a_{t+h} X_{t+h}\right)=\left(\mathbb{E}\left(a_{1}\right)\right)^{2} \gamma_{X}(h) \sim \mathbb{E}\left(a_{1}\right)\right)^{2} C_{\gamma}|h|^{2 d-1}=C_{1}|h|^{2 d-1}
$$

Moreover, given that the ACVF of $X_{t}$ is square summable, the ACVF of $a_{t} X_{t}$ will be square summable as well as

$$
\begin{aligned}
& \sum_{h=-\infty}^{\infty}\left(\operatorname{Cov}\left(a_{t} X_{t}, a_{t+h} X_{t+h}\right)\right)^{2}= \\
& =\left(\mathbb{E}\left(a_{t}\right)\right)^{4} \sum_{h=1}^{\infty}\left(\gamma_{X}(h)\right)^{2}+\left(\mathbb{E}\left(a_{t}\right)\right)^{4} \sum_{h=-\infty}^{-1}\left(\gamma_{X}(h)\right)^{2}+\left(\mathbb{E}\left(a_{t}^{2}\right)\right)^{2}\left(\gamma_{X}(0)\right)^{2} \\
& =\left(\operatorname{Var}\left(a_{1}\right) \gamma_{X}(0)\right)^{2}+2\left(\gamma_{X}(0) \mathbb{E}\left(a_{1}\right)\right)^{2} \operatorname{Var}\left(a_{1}\right)+\left(\mathbb{E}\left(a_{1}\right)\right)^{4} \sum_{h=-\infty}^{\infty}\left(\gamma_{X}(h)\right)^{2}<\infty
\end{aligned}
$$

Hence the spectral density of the process $a_{t} X_{t}$ is guaranteed to exist and will form a Fourier pair with $\operatorname{Cov}\left(a_{t} X_{t}, a_{t+h} X_{t+h}\right)=\gamma_{R T}(h)$. We denote the spectral density of $a_{t} X_{t}$ by $f_{R T}(\omega)$. Furthermore, by inspecting the spectral density of the the process 
we see that it takes on the semi-parametric form of (2.6) as

$$
\begin{aligned}
f_{R T}(\omega) & =\frac{1}{2 \pi n} \sum_{h=-\infty}^{\infty} \gamma_{R T}(h) e^{-i h \omega} \\
& =\frac{1}{2 \pi}\left(\gamma_{R T}(0)+\sum_{h=1}^{\infty} \gamma_{R T}(h) e^{-i h \omega}+\sum_{h=-\infty}^{-1} \gamma_{R T}(h) e^{-i h \omega}\right) \\
& =\frac{1}{2 \pi}\left(\mathbb{E}\left(a_{1}^{2}\right) \gamma_{X}(0)+\left(\mathbb{E}\left(a_{1}\right)\right)^{2} \sum_{h=1}^{\infty} \gamma_{X}(h) e^{-i h \omega}+\left(\mathbb{E}\left(a_{1}\right)\right)^{2} \sum_{h=-\infty}^{-1} \gamma_{X}(h) e^{-i h \omega}\right) \\
& =\frac{1}{2 \pi}\left(\operatorname{Var}\left(a_{1}\right) \gamma_{X}(0)+\left(\mathbb{E}\left(a_{1}\right)\right)^{2} \sum_{h=-\infty}^{\infty} \gamma_{X}(h) e^{-i h \omega}\right) \\
& =\frac{1}{2 \pi}\left(\operatorname{Var}\left(a_{1}\right) \gamma_{X}(0)\right)+\left(\mathbb{E}\left(a_{1}\right)\right)^{2}\left(\frac{1}{2 \pi} \sum_{h=-\infty}^{\infty} \gamma_{X}(h) e^{-i h \omega}\right) \\
& =\frac{1}{2 \pi}\left(\operatorname{Var}\left(a_{1}\right) \gamma_{X}(0)\right)+\left(\mathbb{E}\left(a_{1}\right)\right)^{2} f(\omega) .
\end{aligned}
$$

We note that for $d=0$

$$
\frac{f_{R T}(\theta / n)}{f\left(\omega_{j}\right)} \sim\left(\frac{\operatorname{Var}\left(a_{1}\right) \gamma_{X}(0)}{C_{f} 2 \pi}+\left(\mathbb{E}\left(a_{1}\right)\right)^{2}\right)=C .
$$

Moreover, for $d>0$

$$
\frac{f_{R T}(\theta / n)}{f\left(\omega_{j}\right)} \sim\left(\mathbb{E}\left(a_{1}\right)\right)^{2}\left|\frac{\theta}{2 \pi j}\right|^{-2 d} .
$$

Therefore, given the asymptotic forms of the $\gamma_{R T}(h)$ and $f_{R T}\left(\omega_{j}\right)$ we can analyze the second order characteristics through the ACVF or the spectral density for $d \in$ $[0, .5)$. The function we implement to estimate the spectral density $f_{R T}\left(\omega_{j}\right)$ is the periodogram of the randomly tapered series which take on the following form

$$
I_{n}^{R T}\left(\omega_{j}\right)=\frac{1}{2 \pi n}\left|\sum_{t=1}^{n} a_{t} X_{t} e^{-i \omega_{j} t}\right|^{2}
$$


Moreover, let the randomly tapered periodogram of the "kth" block be defined as

$$
I_{n, k}^{R T}\left(\omega_{j}\right)=\frac{1}{2 \pi l}\left|\sum_{t=1+(k-1) l}^{k l} a_{t} X_{t} e^{-i \omega_{j} t}\right|^{2} .
$$

With the above in mind we now explain the first testing procedure involving the randomly tapered series.

\subsubsection{Asymptotic Distribution Within the First Procedure}

We note that the null and alternative hypothesis are the same for this procedure as first procedure utilizing the cosine bell taper.

Theorem 4.11. Let $X_{t}$ be of the form of Proposition (2.3) with spectral density $f$ of the form of (2.6) where $d \in[0, .5)$.

We define the test statistic as

$$
Q_{n, m}^{R T}(s)=\sum_{j=1}^{s} \frac{I_{n}^{R T}\left(\omega_{j}\right)}{\frac{1}{m} \sum_{k=1}^{m} I_{n, i}^{R T}\left(\omega_{j}^{\prime}\right)} .
$$

a.) If $X_{t}$ is a short memory process in a sense that $d=0$ and

$$
\sum_{k=1}^{\infty}\left|\psi_{k}\right|<\infty
$$

then

$$
Q_{n, m}^{R T}(s) \stackrel{\mathbb{D}}{\longrightarrow} Q(s)=\frac{1}{2} \sum_{j=1}^{s}\left(\left(\left(Z_{1}^{R T}(j)\right)^{2}+\left(Z_{2}^{R T}(j)\right)^{2}\right)\right) .
$$

Where $Q(s) \stackrel{\mathbb{D}}{=} \Gamma(s, 1)$.

b.) If $X_{t}$ is long memory in a sense that $d \in(0, .5)$, then

$$
Q_{n, m}^{R T}(s) \stackrel{\mathbb{P}}{\longrightarrow} \infty
$$


Proof. Part a.) We refer to Part b.) of Proposition (9.23) which allows us to state that

$$
\sum_{j=1}^{s}\left(\frac{I_{n}^{R T}\left(\omega_{1}\right)}{\frac{1}{m} \sum_{k=1}^{m} I_{n, k}^{R T}\left(\omega_{j}^{\prime}\right)}\right) \stackrel{\mathbb{D}}{\longrightarrow} Q^{R T}(s, 0)=Q^{R T}(s),
$$

as $d=0$ and

$$
\begin{aligned}
Q^{R T}(s) & =\frac{1}{\sigma_{R T, j}^{2}} \sum_{j=1}^{s}\left(\left(\sigma_{R T A, j}^{2}\left(Z_{1}^{R T}(j)\right)^{2}+\sigma_{R T B, j}^{2}\left(Z_{2}^{R T}(j)\right)^{2}\right)\right) \\
& =\frac{1}{2} \sum_{j=1}^{s}\left(\left(\left(Z_{1}^{R T}(j)\right)^{2}+\left(Z_{2}^{R T}(j)\right)^{2}\right)\right) .
\end{aligned}
$$

We note $\operatorname{Cov}\left(Z_{1}^{R T}(j), Z_{1}^{R T} k\right)=\operatorname{Cov}\left(Z_{2}^{R T}(j), Z_{2}^{R T}(k)\right)=0, \forall j \neq k$. and $\operatorname{Cov}\left(Z_{1}^{R T}(j), Z_{2}^{R T}(k)\right)=$ $0, \forall j, k$ by Proposition (9.19). Therefore, each variable within

$$
\left(Z_{1}^{R T}(1), \ldots, Z_{1}^{R T}(s), Z_{2}^{R T}(1), ., Z_{2}^{R T}(s)\right) \text { is independent as they are jointly nor- }
$$

mally distributed by Proposition (9.21) and uncorrelated. Hence, $Q^{R T}(s) \stackrel{\mathbb{D}}{=} \Gamma(s, 1)$.

Part b.) By the second part of Proposition (9.23) we have

$$
m^{-2 d}\left(\frac{I_{n}^{R T}\left(\omega_{j}\right)}{\frac{1}{m} \sum_{k=1}^{m} I_{n, k}^{R T}\left(\omega_{j}^{\prime}\right)}\right)=O_{P}(1)
$$

with $d \in(0, .5)$. Hence,

$$
\sum_{j=1}^{s}\left(\frac{I_{n}^{R T}\left(\omega_{j}\right)}{\frac{1}{m} \sum_{k=1}^{m} I_{n, k}^{R T}\left(\omega_{j}^{\prime}\right)}\right)=O_{P}\left(m^{2 d}\right) .
$$

Therefore,

$$
\sum_{j=1}^{s}\left(\frac{I_{n}^{R T}\left(\omega_{j}\right)}{\frac{1}{m} \sum_{k=1}^{m} I_{n, k}^{R T}\left(\omega_{j}^{\prime}\right)}\right) \stackrel{\mathbb{P}}{\longrightarrow} \infty
$$


We denote $\Gamma_{\alpha}(s, 1)$ as the quantile of order $1-\alpha$ for the $\Gamma(s, 1)$ distribution with parameters $s$ and 1 . The critical region for the test to distinguish between short and long memory is $\left\{Q_{n, m}^{R T}(s)>\Gamma_{\alpha}(s, 1)\right\}$. Moreover,

$$
P\left(Q_{n, m}^{R T}(s)>\Gamma_{\alpha}(s, 1) \mid H_{0}\right) \rightarrow \alpha
$$

Hence, the test is correct. Furthermore, under the alternate hypothesis the test is consistent as the results in Part b.) of the above theorem directly imply

$$
P\left(Q_{n, m}^{R T}(s)>\Gamma_{\alpha}(s, 1) \mid H_{1}\right) \rightarrow 1
$$

since $Q_{n, m}^{R T}(s) \stackrel{\mathbb{P}}{\longrightarrow} \infty$ under the alternative hypothesis. We now speak of the asymptotic distribution for the second procedure.

We now proceed to discuss our methodology used to assess these statistics.

\subsection{Testing Procedure Methodology}

To assess the performance of our two statistical procedures we perform an array of Monte Carlo simulations similar to Gromykov et al.(2018) and Giraistis et al.(2003) by generating P-value plots and Empirical Power plots. These are performed to help determine the optimal window parameters and demonstrate the efficacy of our tests. This is achieved by evaluating the accrued empirical size and empirical power results of our proposed test statistics underneath the null and alternative hypotheses, respectively. In both of our procedures the theoretical distribution used to generate the p-values represents the asymptotic distribution given under the null hypothesis 
for each test statistic. In the first procedure this is known prior to performing the procedure. However, in the case of the second procedure the limiting distribution corresponds to the value $d$, which is typically unknown in practice. Therefore, we use the asymptotic distributions with the actual value of $d$ used to simulate the array of observation and also the asymptotic distribution corresponding to the estimated value of $d$ (rounded off to .001). We now state in step-by-step form how we perform our simulations for both procedures under the the null and alternative hypotheses.

- The window parameters, sample size, and innovation distribution assumptions are specified.

- For each simulation the sample is drawn and the data is used to compute the test statistics.

- The above step is repeated 5000 times with each simulation's accrued test statistic value being retained.

- The array of 5000 simulated test statistic values are then plugged into the corresponding asymptotic distribution and then subtracted off from 1 which generates a set of the p-values.

- The empirical CDF is then computed for the p-values by using R's "ecdf" function. 
For the both procedures we perform the simulations with sample sizes 100,1000, 5000, and 10000. The innovations used are denoted as $\epsilon_{t}$ and satisfy

$$
\mathbb{E}\left(\epsilon_{t}\right)=0, \quad \mathbb{E}\left(\epsilon_{t}^{r}\right)=\sigma_{\epsilon}^{2}, \quad \mathbb{E}\left(\epsilon_{t}^{4}\right)<\infty .
$$

We also note that the innovations can be from anyone of the following distributions

$$
\{\exp (1)-1, N(0,1),(\log (N(0,1))-\sqrt{e}) / \sqrt{e(e-1)})\} .
$$

Moreover, we also note that the values of the window parameters we assess are $m=$ $n^{3}, n^{4}, n^{.5}, n^{6}$, and $s=1,2,3,4,5,7$.

\subsubsection{First Procedure}

Underneath the null hypothesis we generate $X_{t}$ from a $\mathrm{AR}(1)$ process of the form

$$
X_{t}=\phi X_{t-1}+\epsilon_{t}, \quad|\phi|<1 .
$$

Under the alternative hypothesis we generate $X_{t}$ from a long memory process of the form

$$
(1-\mathbb{B})^{d} X_{t}=\epsilon_{t}
$$

where $d \in(.1, .2, .3, .4, .45, .49)$ and $\mathbb{B}$ is the backshift operator.

For the first procedure we state the functional forms of each test statistic we evaluate. The test statistic we have developed with the cosine bell taper takes on two forms within this procedure.

$$
Q_{n, m}^{T}(s)=\sum_{j=1}^{s} \frac{I_{n}^{T}\left(\omega_{j}\right)}{\frac{1}{m} \sum_{k=1}^{m} I_{n, k}^{T}\left(\omega_{j}^{\prime}\right)},
$$


and

$$
Q_{n, m}^{T *}(s)=\sum_{j=1}^{s} \frac{I_{n}^{T}\left(\omega_{j}\right)}{\frac{1}{m} \sum_{k=1}^{m} I_{n, k}^{T}\left(\omega_{j}\right)} .
$$

Below are the formulas for the statistic constructed with the random tapers

$$
Q_{n, m}^{R T}(s)=\sum_{j=1}^{s} \frac{I_{n}^{R T}\left(\omega_{j}\right)}{\frac{1}{m} \sum_{k=1}^{m} I_{n, k}^{R T}\left(\omega_{j}^{\prime}\right)}
$$

and

$$
Q_{n, m}^{R T *}(s)=\sum_{j=1}^{s} \frac{I_{n}^{R T}\left(\omega_{j}\right)}{\frac{1}{m} \sum_{k=1}^{m} I_{n, k}^{R T}\left(\omega_{j}\right)} .
$$

We refer to the following as the $Q_{n, m}^{*}(s)$ statistic which is from Gromykov et al.(2018)

$$
Q_{n, m}^{*}(s)=\sum_{j=1}^{s} \frac{I_{n}\left(\omega_{j}\right)}{\frac{1}{m} \sum_{k=1}^{m} I_{n, k}\left(\omega_{j}\right)} .
$$

The LR and VS statisitics are also assessed within this array of simulations.

\subsubsection{Second Procedure}

We now present the results from our second procedure. Under the null hypothesis we generate $X_{t}$ from the following process

$$
(1-\mathbb{B})^{d} X_{t}=\epsilon_{t}
$$

where $d \in(-.49,-.4,-.3,-.2,-.1,-.01, .01, .1, .2, .3, .4, .49)$.

For the alternative hypothesis we generate $X_{t}$ from a random walk of the form

$$
X_{t}=X_{t-1}+Y_{t}
$$


or from a process with a linear deterministic trend embedded within it given in the form of

$$
X_{t}=.005 t+Y_{t}
$$

Where $Y_{t}$ follows a form satisfying Proposition (2.3) with spectral density of the form (2.6) where $d \in(-.5, .5)$. We note that the coefficient for the deterministic trend is one of the values implemented for the linear trend within Giraistis et al.(2006).

There are three statistics evaluated within this section. We define these below starting with the cosine bell taper with the first ordinate removed.

$$
Q_{n, m}^{T^{\prime}}(s, d)=m^{-2 d} \sum_{j=2}^{s} \frac{I_{n}^{T}\left(\omega_{j}\right)}{\frac{1}{m} \sum_{k=1}^{m} I_{n, k}^{T}\left(\omega_{j}^{\prime}\right)} .
$$

Below is the formula for the statistic with the cosine bell taper with the first ordinate remaining

$$
Q_{n, m}^{T}(s, d)=m^{-2 d} \sum_{j=1}^{s} \frac{I_{n}^{T}\left(\omega_{j}\right)}{\frac{1}{m} \sum_{k=1}^{m} I_{n, k}^{T}\left(\omega_{j}^{\prime}\right)} .
$$

For the $Q_{n, m}^{T}(s, d)$ we estimate the values of $d$ with the GPH estimator through the untapered series which we refer to as $d_{G P H}$. We also inspect the results from obtaining the GPH estimator with a the cosine bell taper applied to the series which we refer to as $d_{G P H}^{T}$. It has been suggested by Hurvich \& Ray (1995) to remove the first periodogram ordinate from the log periodogram regression to generate an estimator for $d$ when $d>.5$. Although underneath the null conditions of this procedure this assumption is satisfied and one will not have to worry about the issues proposed here. We still inspect the P-value plots to see what employing such constraints would yield 
on the performance of the statistic. Therefore, with the cosine bell taper used to generate the GPH estimator of $d$ we also inspect how removing the first periodogram ordinate from the log periodogram regression impacts the performance of the test statistics within this procedure. We denote this estimator as $d_{G P H}^{T^{\prime}}$. We now speak of the limiting distributions used within each procedure.

\subsection{Limiting Distribution}

Aside from the first procedure with the random taper, the test statistics for both of our procedures have a limiting distribution that is not available in a closed form representation and therefore, will need to be generated numerically. As depicted in each procedure, the sum of the tapered periodogram ordinates are sums of weighted Chi-squares random variables with the weights being given in the form of the variance and covariances of each of the components. Therefore, these weights are functions of $j$ and $d$. Moreover, for each value of $d$ between $[-.5, .5]$ with incremental values of .001 and values of $j=1, . ., 14$ we numerically compute the weights. Furthermore, for each required procedure we generate an approximation of the limiting distribution by taking the empirical distribution function of 100000 simulations of weighted Chisquare random variables by utilizing a quadratic form composed with an i.i.d $N_{2 s}(0,1)$ vector with the covariance matrix of the components being the real symmetric matrix.

For instance, with the $Q_{n, m}^{T}(s)$ and the $Q_{n, m}^{T}(s, d)$ statistics the quadratic form we implement is 


$$
Z^{\prime} \Sigma_{2 s, 2 s, d}^{T} Z=Q_{k}^{T}(s, d)
$$

where $k \in(1, \ldots \ldots, 100000)$ and $Z$ is an i.i.d standard Gaussian random vector of $2 s$ components. Moreover, the covariance matrix we use in the computation is from the random vector

$$
\left(\frac{\sigma_{A, 1}}{\sqrt{L_{1, T}(d)}} Z_{1}(1), \ldots, \frac{\sigma_{A, s}}{\sqrt{L_{s, T}(d)}} Z_{1}(s), \frac{\sigma_{B, 1}}{\sqrt{L_{1, T}(d)}} Z_{2}(2) . ., \frac{\sigma_{B, s}}{\sqrt{L_{s, T}(d)}} Z_{2}(s)\right) .
$$

We note that the components in the vector above are from Proposition (4.2). We then take the empirical CDF of the generated values of $Q_{k}^{T}(s, d)$ for each $k$ and treat this as the limiting cumulative distribution function for the $Q_{n, m}^{T}(s, d)$ statistic. Furthermore, the limiting distribution for the $Q_{n, m}^{T^{\prime}}(s, d)$ statistic is analogous to the above however, the index $j$ is changed to $j=2, \ldots . ., s$ therefore, the covariance matrix is the same as the one given above with the variance and covariances referring to the first Fourier frequency removed. Moreover, one should note that

$$
Q_{k}^{T}(s, d)=\frac{1}{2} \sum_{j=1}^{s}\left(Z_{1}^{2}(j)+Z_{2}^{2}(j)\right)+\frac{R_{j, T}}{L_{j, T}(d)}\left(\left(Z_{1}^{2}(j)-Z_{2}^{2}(j)\right)\right) .
$$

We note that for this procedure the amount of observations drawn is 100000 . We would also like to state that initially we started with 1000000 observations. However, due to the large amount of storage these matrices encompassed, performing simulations with these objects became quite computationally expensive and therefore, we decided to reduce the sample size to $n=100000$.

Moreover, for the $Q_{n, m}^{R T}(s)$ test, we use a $\Gamma(s, 1)$ distribution as the limiting dis- 
tribution under the null. We proceed now to the next section which explains our findings.

\section{$5 \quad$ First Procedure Findings}

Within this section we describe the results from our developed test statistics from an array of Monte Carlo simulations by visually evaluating their induced P-value and Empirical Power plots.

\subsection{Null Hypothesis}

We now proceed to review our findings under the null hypothesis. The first statistic's performance we analyze is the $Q_{n, m}^{T *}(s)$ statistic.

\subsection{1 $\mathrm{Q}_{n, m}^{T}(s)$}

We evaluate first the performance of $Q_{n, m}^{T}(s)$ find that under all sample sizes and tested block amounts greater than $m=n^{3}$ the test incurs substantial bias for almost all of the tested $\phi$ and $s$ values. At given values of $m$ the window parameter $s$ appears to behave uniformly in a sense that increasing the value of $s$ diminishes the tests ability to obtain a precise estimate of theoretical size.

Therefore, following a similar approach as Gromykov et al.(2018) we fix $m$ at a perceived optimal value of (here being $m=n^{3}$ ) and evaluate the window parameter $s$. It appears that decreasing the sample length decreases the precision of the test as the 

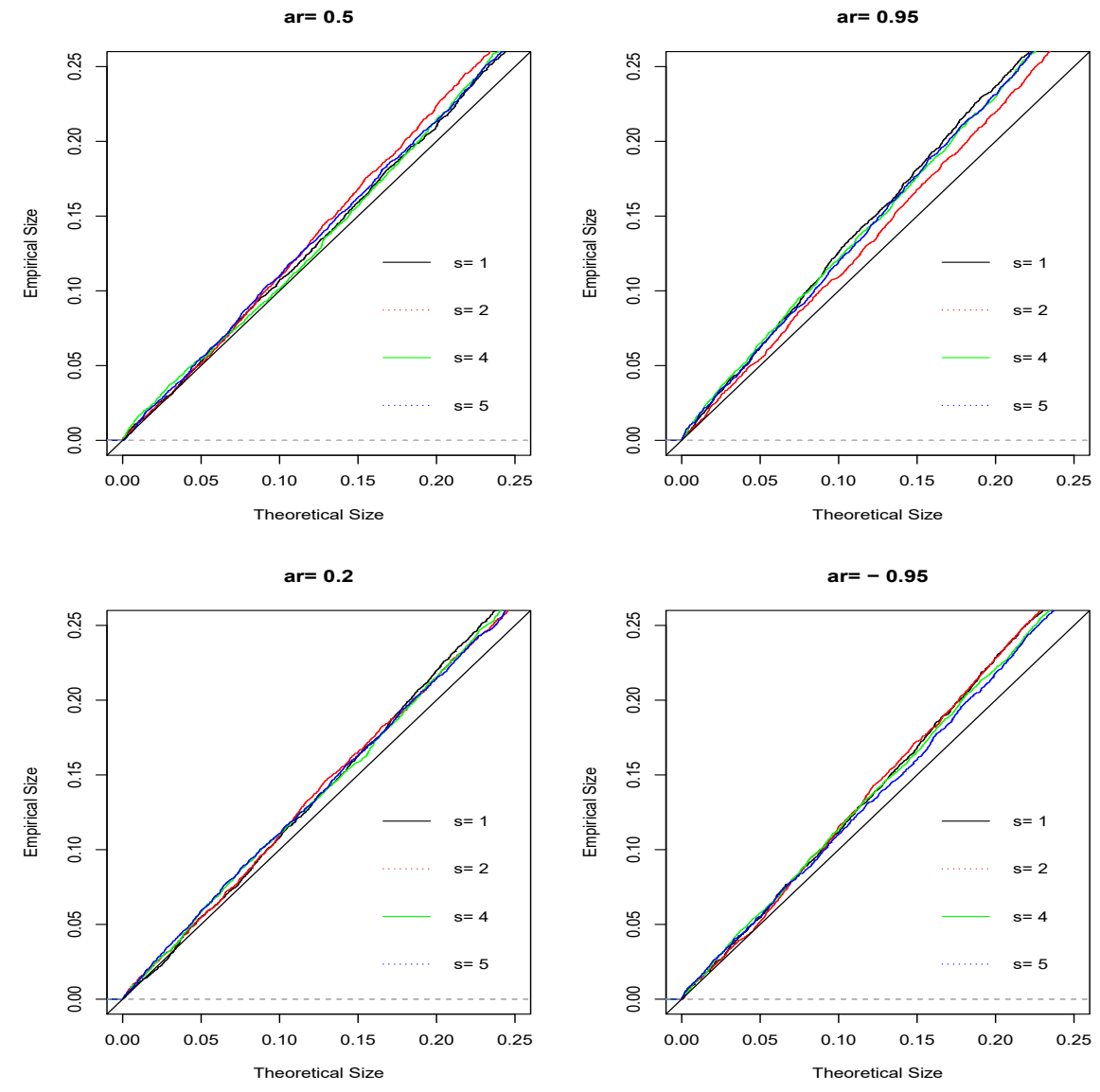

Figure 3: $Q_{n, m}^{T *}(s) n=5000, m=n^{3}, X_{t}=\phi X_{t-1}+\epsilon_{t}, \epsilon_{t} \stackrel{i . i . d}{\sim}(\exp (1)-1)$ 

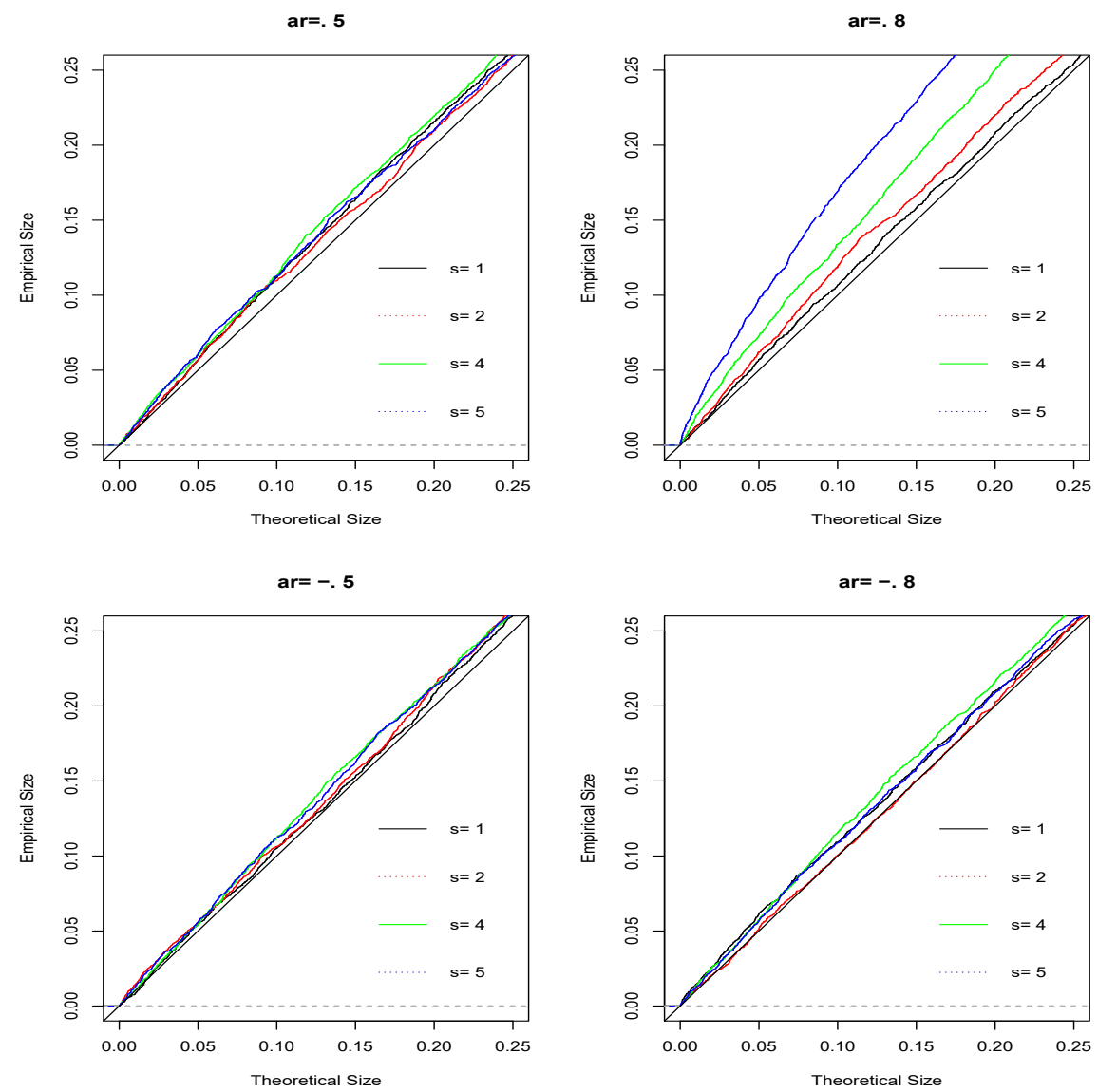

Figure 4: $Q_{n, m}^{T}(s), n=10,000, m=n^{4}, X_{t}=\phi X_{t-1}+\epsilon_{t}, \epsilon_{t} \stackrel{i . i . d}{\sim} \exp (1)-1$.

empirical get larger. We find that $Q_{n, n^{3}}^{T *}(s)$ does not incur any bias for large sample sizes for all tested $\phi$ and values of $s$ under Non-Gaussian and Gaussian innovations. For instance, even for small sample sizes the test performs well as the statistic yields low values of empirical size for all tested $\phi \in(-.95, .95)$. We find as well that for all sample sizes the test performs well at obtaining the correct theoretical size at tested $\phi \leq .95$ and $s \leq 5$. However, for medium and large sample sizes one can increase $s$ while retaining precision in empirical size. 
We find that by changing the Fourier frequency to the length of each block used in the averaged periodogram yields a statistic that generates similar results to the $Q_{n, m}^{T *}(s)$. For the $Q_{n, m}^{T}(s)$ test we find that the optimal block amount is $m=n^{4}$. Therefore, we fix $m$ and evaluate the statistic's performance accordingly. We find that the statistic performs robustly for all tested values $\phi$ away from the upper bound of $1(\phi \leq .8)$ and values of $s \leq 7$ for large sample sizes. For medium and small sample sizes we recommend using smaller values of $s$. We now review the findings from implementing the random taper.

\section{$5.1 .2 \quad Q_{n, m}^{R T}(s)$}

When evaluating the $Q_{n, m}^{R T}(s)$ we find that similar to the previous results with the cosine bell taper, the test statistic's performance improves considerably when the Fourier frequency of the averaged periodogram is implemented with the length of the block rather than the sample length. For instance, in the $Q_{n, m}^{R T *}(s)$ statistic we find that for $\phi$ close to -1 , the statistic produces results that allude to it being a biased procedure. By implementing the correction to the Fourier frequency we find these results to be nullified. Moreover, we find that the optimal block value is $m=n^{4}$ as the other tested block values yield some form of undesirable results.

Therefore, we fix $m$ and focus on determining an appropriate value of $s$ while analyzing how the statistic behaves under the simulated conditions. We find that as $s$ increases the precision in empirical size is lost. We find that by decreasing the sample 

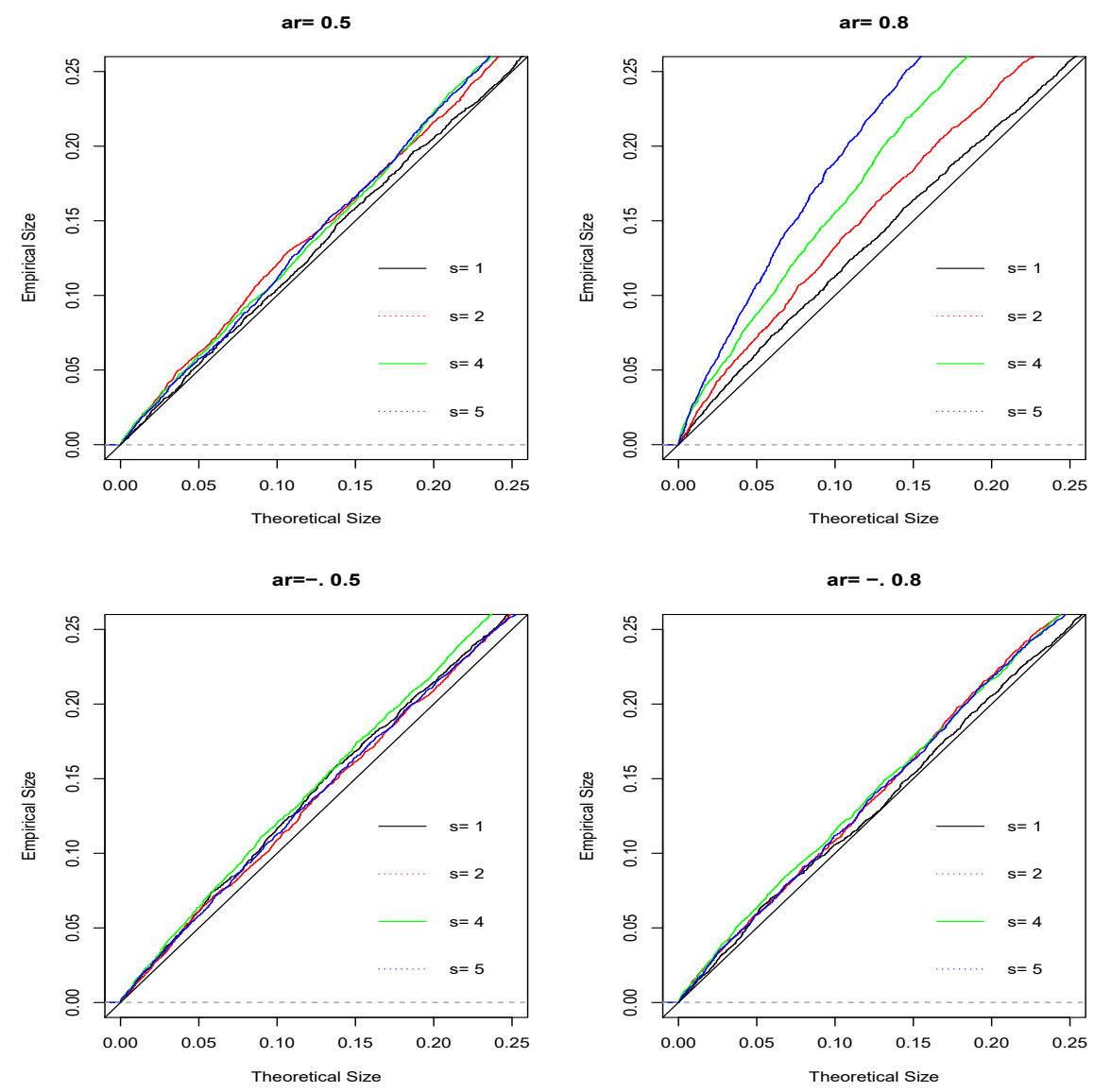

Figure 5: $Q_{n, m}^{R T}(s), n=10000, m=n^{4}, X_{t}=\phi X_{t-1}+\epsilon_{t}, \epsilon_{t} \stackrel{i . i . d}{\sim} \exp (1)-1$.

From this figure we can see that the test yields robust results for large sample sizes as it yields low values of empirical size and never incurs bias. 
size the test becomes less accurate in empirical size. For instance, it can be seen in Figure (6) that for small to medium sample sizes that $s \leq 2$ the test yields desirable results for $\phi<.5$. For $\phi \geq .5$ the first two values of $s$ appear to be well behaved. The test appears to increase in empirical size as $\phi \rightarrow 1$ and appears to be precise for all tested values of $s$ as $\phi \rightarrow-1$. We find these results to remain essentially the same when altering the innovations between Gaussian and Non-Gaussian. We also find that by changing between the random taper from (4.5) does not alter the performance of the statistic to a noticeable extent.

We now review the findings from the simulations under the alternate hypothesis.

\subsection{Alternative Hypothesis}

Fixing $m$ to be at each statistics optimal amount given in the first part of this findings section we find that the empirical power of the $Q_{n, m}^{T}(s), Q_{n, m}^{R T}(s)$, increases as $s$ increases. We also find that by increasing the sample size increases the empirical power as well. Overall, as $d \rightarrow .5$ we find that the statistics' empirical power increases in uniform fashion for each value of $s$ as one can see in Figure (7). We find that the $Q_{n, m}^{R T}(s)$ to have greater power than the $Q_{n, m}^{T}(s)$ statistic. This is expected as the $Q_{n, m}^{T}(s)$ statistic appears to be more efficient in generating more precise empirical size. Therefore, the findings above in conjuction with those within under the null hypothesis in regards to $\mathrm{s}$, it appears that by fixing $m$ and adjusting $s$, one can alter the empirical size and empirical power. 

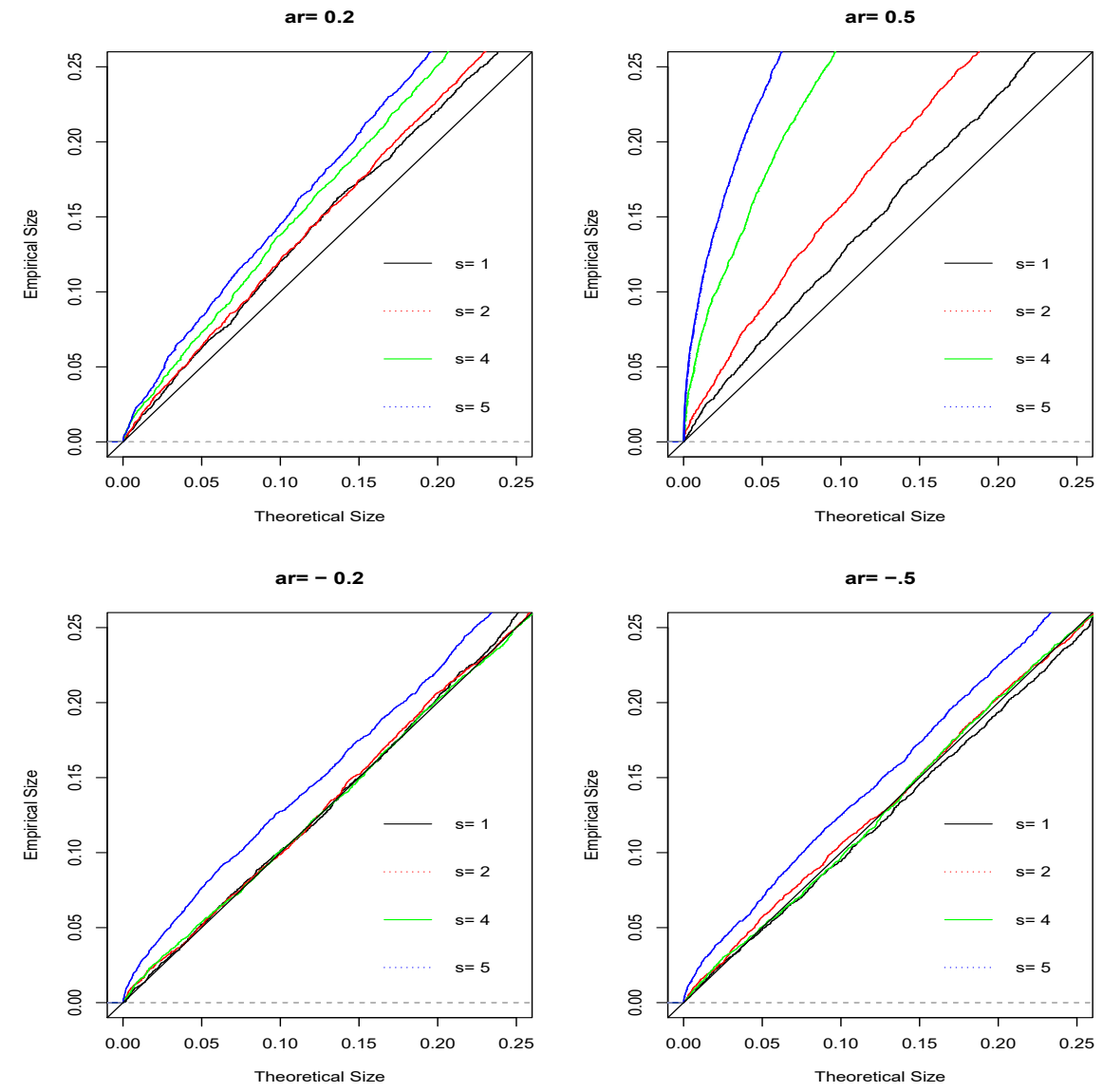

Figure 6: $Q_{n, m}^{R T}(s) n=1000, m=n^{4}, \mathrm{X}_{t}=\phi X_{t-1}+\epsilon_{t}, \epsilon_{t} \stackrel{i . i . d}{\sim} \log N(0,1)$. 

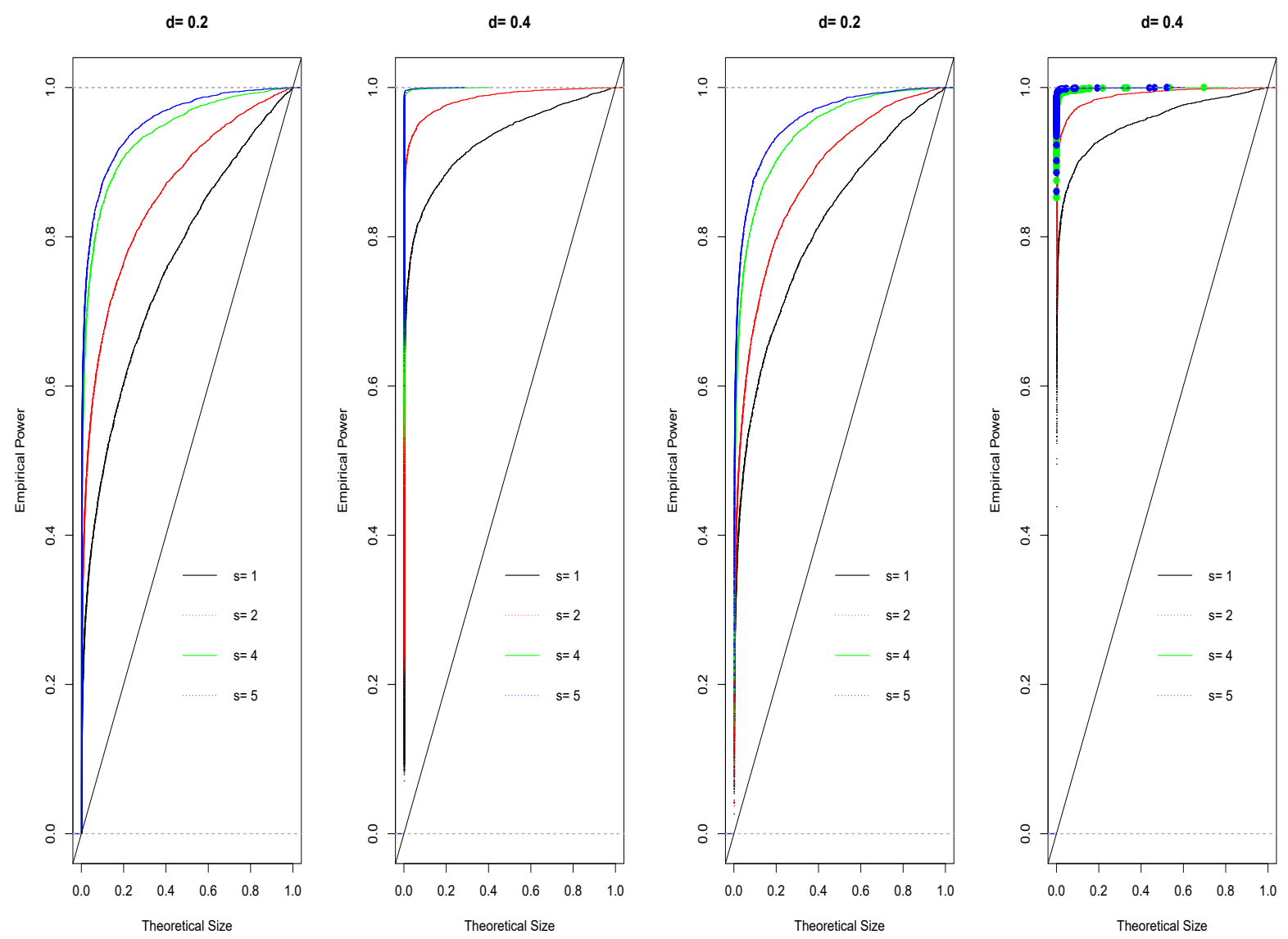

Figure 7: Left: $Q_{n, m}^{R T}(s), n=5000, m=n^{4}, X_{t}=(1-B)^{d} \epsilon_{t}, \epsilon_{t} \stackrel{\text { i.i.d }}{\sim} N(0,1)$. Right:

$$
Q_{n, m}^{T}(s) n=10,000, m=n^{4}, X_{t}=(1-B)^{d} \epsilon_{t}, \epsilon_{t} \stackrel{i . i . d}{\sim} \exp (1)-1
$$




\subsection{Comparision}

\subsubsection{Null Hypothesis}

We now compare the performance of the $Q_{n, m}^{T}(s)$ with the $Q_{n, m}(s)$ with the LR and VS test under the null and alternate hypothesis. For this analysis we choose the value of $\mathrm{s}$ for the $Q_{n, m}^{T}(s)$ to be $s=1$ and $m=n^{4}$. For the $Q^{n, m}(s)$ test we choose the parameter values $s=2, m=n^{5}$ which are the ones chosen in the comparision perfromed within Gromykov et al.(2018.). For the LR and VS tests the chosen value of the bandwidth parameter are taken directly from the suggested optimal amounts in Lobato \& Robinson (1998) and Giraistis et al.(2003), respectively.

When performing the comparision under the null hypothesis with $Q_{n, m}^{T}(s)$ when $s$ is chosen to be lower order values of such as $s=1,2$ as the statistic never incurs any bias and is more precise in terms of achieving empirical size than the other statistics. We can see that the VS statistic becomes biased for $\phi=-.2,-.5,-.8,-.95$. We also find that for $\phi=.5, .8, .95$ the VS statistic seems to achieve less precision in empirical size especially at $\phi=.8, .95$ as its empirical size increases substantially. We also note that at the non-stationary bound of 1 , the $Q_{n, m}^{T}(s)$ and the $Q_{n, m}(s)$ are the most robust to increases in empirical sizes as one can see in Figure (8). For small sample sizes the VS statistic exhibits similar behaviour to that of its asymptotic counterpart (ie when $n=10000$ ) that being biased values for negative $\phi$ values and less empirical precision for $\phi=.5, .8, .95$ then the other statistics in the case of Gaussian and nonGaussian innovations. At small and medium sample sizes we find that frequency 

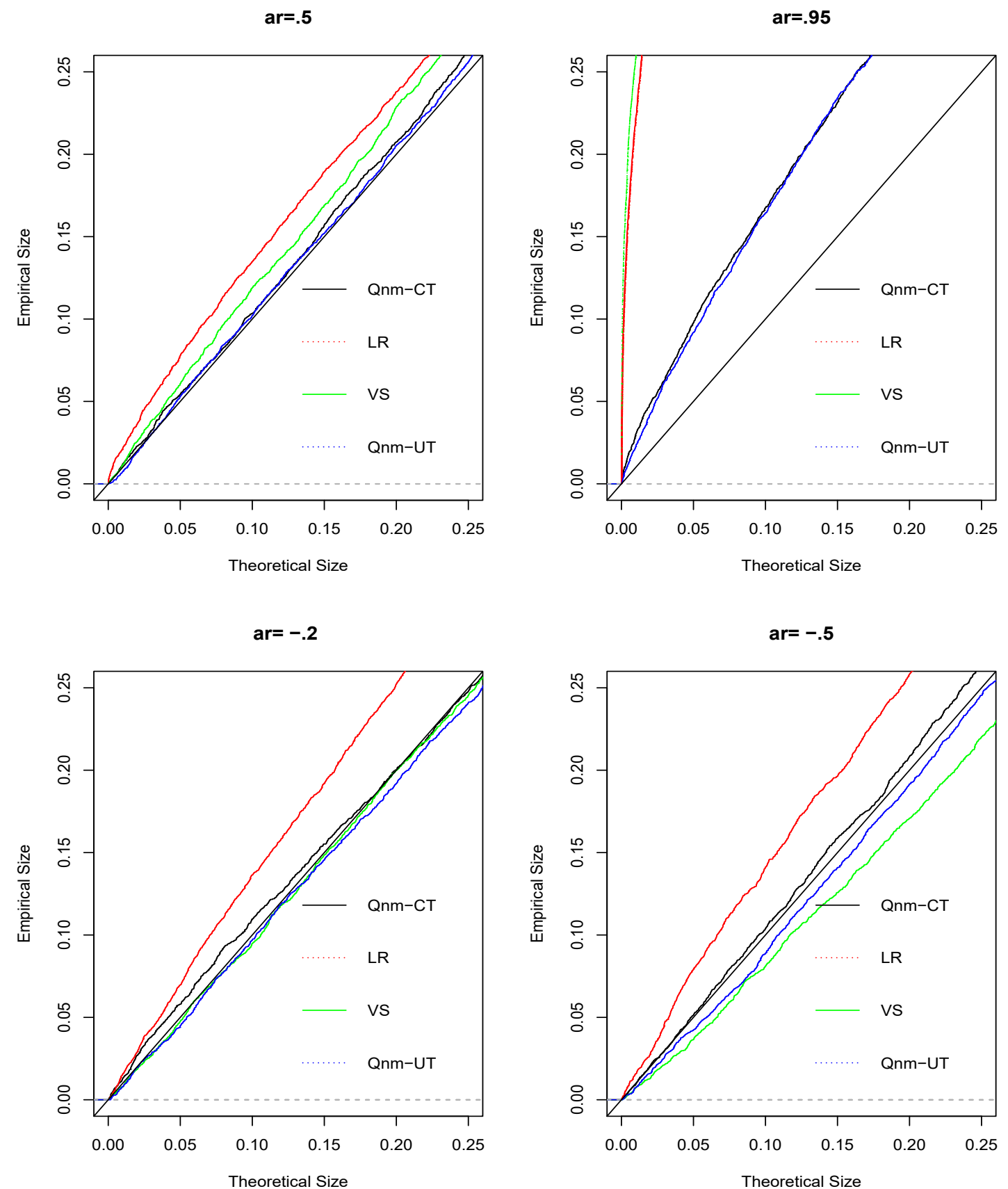

Figure 8: $\quad Q_{n, m}^{T}(s), Q_{n, m}(s), V S, \& \quad L R, n=10000, X_{t}=\phi X_{t-1}+\epsilon_{t}, \epsilon_{t} \stackrel{i . i . d}{\sim}$ $(\log (N(0,1))-\sqrt{e}) / \sqrt{e(e-1)}))$ 
domain statistics are the most robust in their empirical size estimates as the VS statistic appears to increase substantially in empirical size as $\phi \rightarrow 1$ and becomes biased as $\phi \rightarrow-1$.

For $\mathrm{n}=100$, for non-Gaussian and Gaussian innvations we find that the $Q_{n, m}^{T}(s)$ is the only statistic that behaves moderately well underneath the array of simulated conditions. This stems from the fact that the other statistics are biased at $\phi<0$ and for $\phi=.5, .8, .95$ the generated empirical sizes that are much larger than the corresponding theoretical sizes. Whereas, the $Q_{n, m}^{T}(s)$ statistic performs somewhat accurately.

\subsubsection{Alternative Hypothesis}

In the case of long memory we find that the LR and VS statistics have almost uniformly higher power for differing values of $d$ across sample sizes from $n=1000,5000,10000$, than the $Q_{n, m}^{T}(s)$ and the $Q_{n, m}(s)$ statistics as Figure (9) depicts. The $Q_{n, m}^{T}(s)$ accrues slightly less empirical power than the $Q_{n, m}(s)$ for almost all sample sizes and

values of $d$. In the case of $\mathrm{n}=100$ we find that the $Q_{n, m}^{T}(s)$ has the highest empirical power across the tested values of $d$ compared to the other statistics. Taking this into conjunction with the findings for very small sample sizes of $n=100$ this potentially suggests that this test is the most reliable for very small sample sizes. We find that altering the innovations assumption does not change any of the previously mentioned results to a considerable extent. 

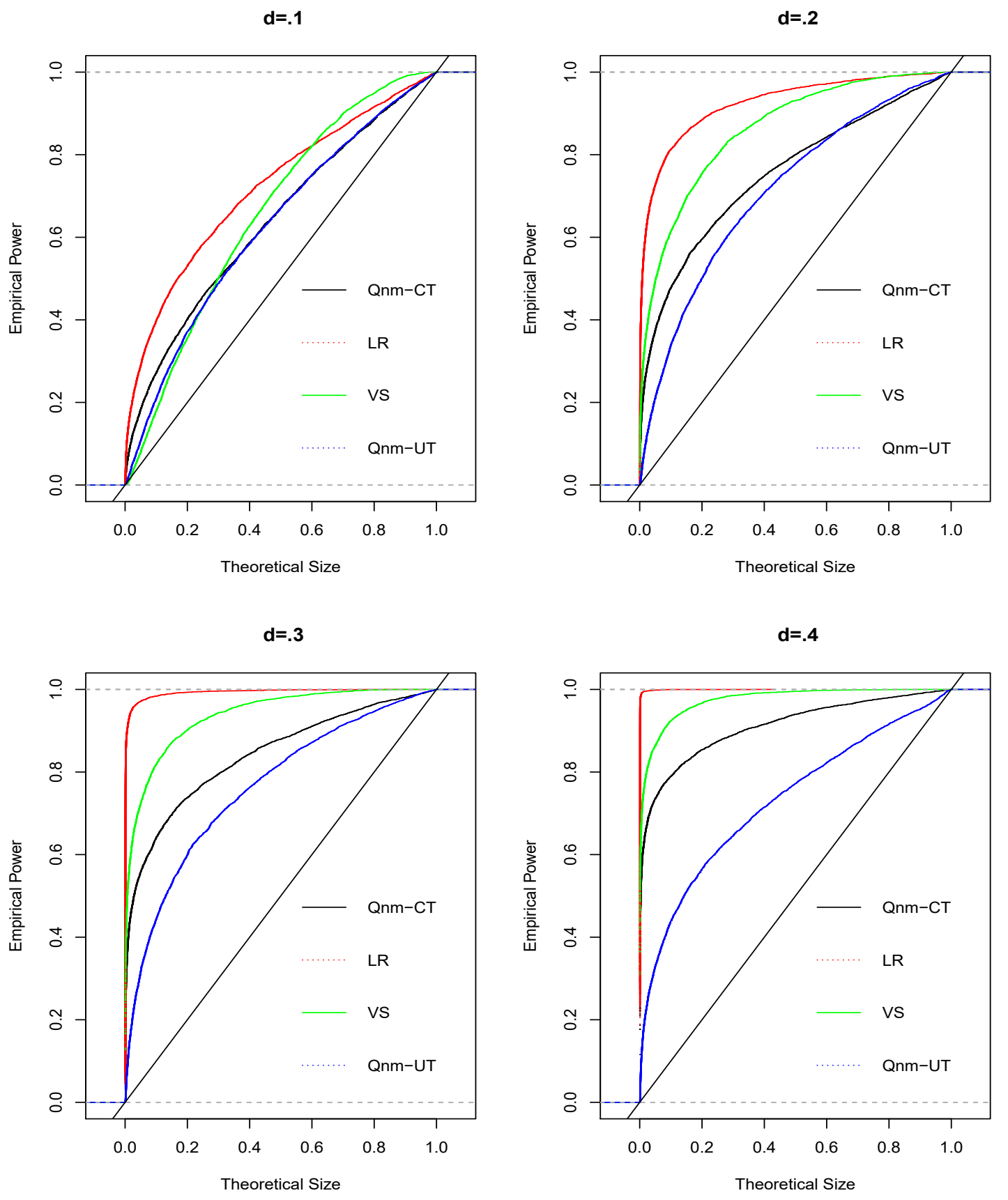

Figure 9: $Q_{n, m}^{T}(s), Q_{n, m}(s), V S, \& L R . n=1000, X_{t}=(1-B)^{d} \epsilon_{t}, \epsilon_{t} \stackrel{\text { i.i.d }}{\sim} N(0,1)$. 


\subsection{Conclusion}

Underneath the null hypothesis we find that the statistics made with the cosine bell and class of random tapers yield promising results. We find that by fixing $m$ we obtain robust empirical size values under a large array of suitable conditions. Moreover, by altering the Fourier frequency in the averaged periodogram we find the performance improves considerably for both statistics. It appears that by increasing $s$ and the sample size the accrued empirical size by each statistic is increased. We note that the statistics perform well apart from $\phi$ being close to 1 . The $Q_{n, m}^{T}(s)$ yields more precise empirical size when compared against the $Q_{n, m}^{R T}(s)$ statistic. Under the alternate hypothesis we find the $Q_{n, m}^{R T}(s)$ to yield higher empirical power out of the two. We also find the results to hold for the $Q_{n, m}^{R T}(s)$ regardless of the taper implemented from the class given in (4.5). When comparing the statistics to the $Q_{n, m}(s)$, LR, and VS we find that the frequency domain statistics appear to perform more robustly in obtaining empirical size than the time domain statistic. We also find that the $Q_{n, n^{4}}^{T}(1)$ performs very well in obtaining empirical size however, in obtaining empirical power all of the other statistics appear to accrue larger amounts for each value of $d$. For very small sample sizes we also find that the $Q_{n, n^{4}}^{T}(1)$ is the only statistic moderately well behaved. We find all of the previously mentioned results to remain true in the case of Gaussian or Non-Gaussian innovations.

We now proceed to depict the findings within the second procedure of our research. 


\section{Second Procedure Testing Results}

As stated previously, in the second procedure the memory parameter $d$ needs to be estimated. This adds an extra layer of uncertainty to the test as the limiting distribution that is used to generate the p-values corresponds to the estimated value of $d$. Therefore, we have analyzed each statistics performance with the limiting distribution corresponding to the estimated $d$ and the true value of $d$. We find that using either the estimated or true value does not substantially alter the results in the P-value plots and the Empirical Power plots. Hence, we have presented the results with limiting distribution based off of the implemented value of $d$.

\subsection{Null Hypothesis}

\subsection{1 $\mathbf{Q}_{n, m}^{T}(s, d)$}

Starting with the $Q_{n, m}^{T}\left(s, d_{G P H}\right)$ statistic, we find that the optimal amount of blocks $\mathrm{s} n=.3$. Similar to the previous analysis within this procedure, we find that $s=1,2$ produces undesirable results underneath Gaussian and Non-Gaussian innovations. Therefore, we recommend using $s=3,4,5$ for small to medium sample sizes. When $n$ becomes one can increase $s$ without jeopardizing the statistics ability to yield optimal empirical size levels.

We find that by tapering the series within the GPH estimator with the cosine bell taper induces undesirable results for almost all tested values of the windor parameters. Therefore, it is not recommended. We also find that when implementing the 
cosine bell taper and removing the first peridogram ordinate in the GPH estimator on the $Q_{n, m}^{T}$ statistic the results that are quite detrimental and essentially nullify any plausibility of this statistic being implemented and used for this procedure.

\subsection{2 $\mathbf{Q}_{n, m}^{T^{\prime}}\left(s, d_{G P H}^{T}\right)$}

By utilizing the $Q_{n, m}^{T^{\prime}}\left(s, d_{G P H}\right)$ statistic in comparison to the $Q_{n, m}^{T}$ statistic, we find that the optimal value of $m$ is $n^{3}$. We also find the obscure behaviour of $Q_{n, m}^{T}\left(s, d_{G P H}\right)$ at $s=1,2$ is not apparent in the $Q_{n, m}^{T^{\prime}}\left(s, d_{G P H}\right)$. Moreover, the statistic behaves in a controlled manner as we find that empirical size increases as $n$ or $s$ increase. For all tested values of $d \in(-.4, .4]$ the statistic appears to perform quite well for all sample sizes and $s \leq 5$. We find that medium and large sample sizes the statistic obtains low empirical size values even as $d \rightarrow .5$ and $s \leq 5$ as can be seen in Figure (10).

When evaluating the $Q_{n, m}^{T^{\prime}}(s, d)$ statistic with the $d_{G P H}^{T}$ estimator of $d$ we find that the performance is worsened as for differing values of $d$ the statistic appears yield results that allude to the procedure being biased. Moreover, when evaluating the $Q_{n, m}^{T^{\prime}}\left(s, d_{G P H}^{T^{\prime}}\right)$ we find that the statistic yields similar results eluding to the procedure being biased for values of $d$ close to -.5 . Therefore, it appears that the optimal form of the $Q_{n, m}^{T^{\prime}}(s, d)$ is with the untapered GPH estimator. 

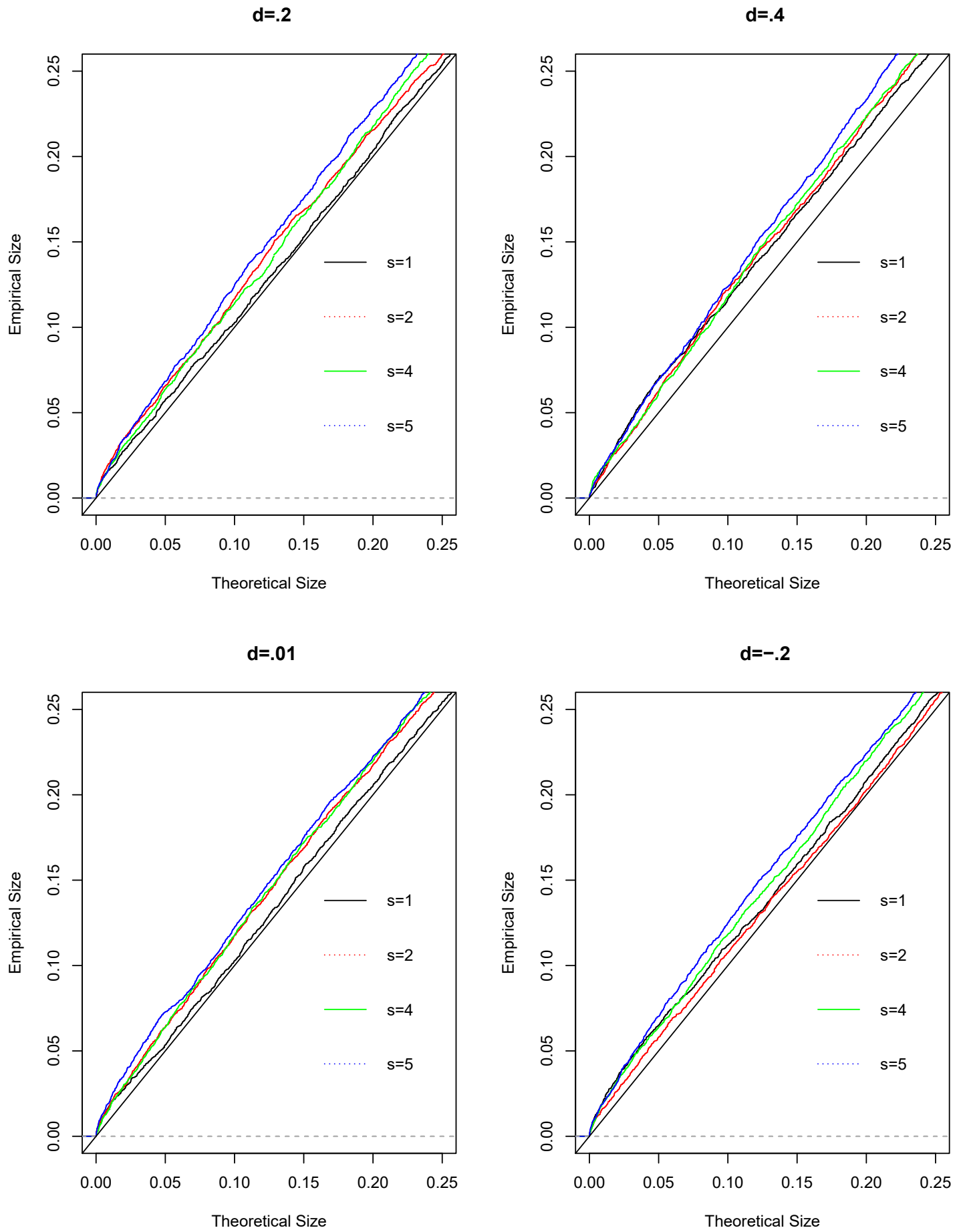

Figure 10: $Q_{n, m}^{T^{\prime}}\left(s, d_{G P H}\right), n=5000, m=n^{3}, X_{t}=(1-B)^{d} \epsilon_{t}, \epsilon_{t} \stackrel{\text { i.i.d }}{\sim} \exp (1)-1$. 


\subsection{Alternative Hypothesis}

Within this section we evaluate the performance of our statistics underneath a set of conditions that satisfy the alternative hypothesis to test the empirical power performance of our statistics. In this section we test note that we fix $m=n^{3}$ for each statistic.

For $Q_{n, m}^{T}\left(s, d_{G P H}\right)$ we find that by using $s=1$ a bias occurs under alternate conditions with a random walk. This further alludes to this statistic being inappropriate to implement for this procedure. For $Q_{n, m}^{T^{\prime}}\left(s, d_{G P H}\right)$ we find that the empirical power is increasing as $s$ increases. We note that this statistic performs very robustly for medium to large sample sizes as at almost all values of $s$ we find that the empirical power is quite high. However, for smaller sample sizes we find that the empirical power diminishes from this level quite substantially.

Overall, we find their are issues with utilizing the $Q_{n, m}^{T}\left(s, d_{G P H}\right)$ that could be related to the use of the first periodogram ordinate as initially suggested. Therefore, taking the above sentiment in conjunction with the theoretical concerns presented by Hurvich \& Ray (1995) underneath certain forms of non-stationarity, we do not recommend using this statistic for this procedure.

In the case of a random walk we find that $Q_{n, m}^{T^{\prime}}\left(s, d_{G P H}\right)$ appears to yield decent empirical power. This is intuitive given the empirical size precision of the former statistic under the null hypothesis. Another finding can be seen in Figure (11) which

depicts that in the case of a linear trend we find that the $Q_{n, m}^{T^{\prime}}\left(s, d_{G P H}\right)$ is very 

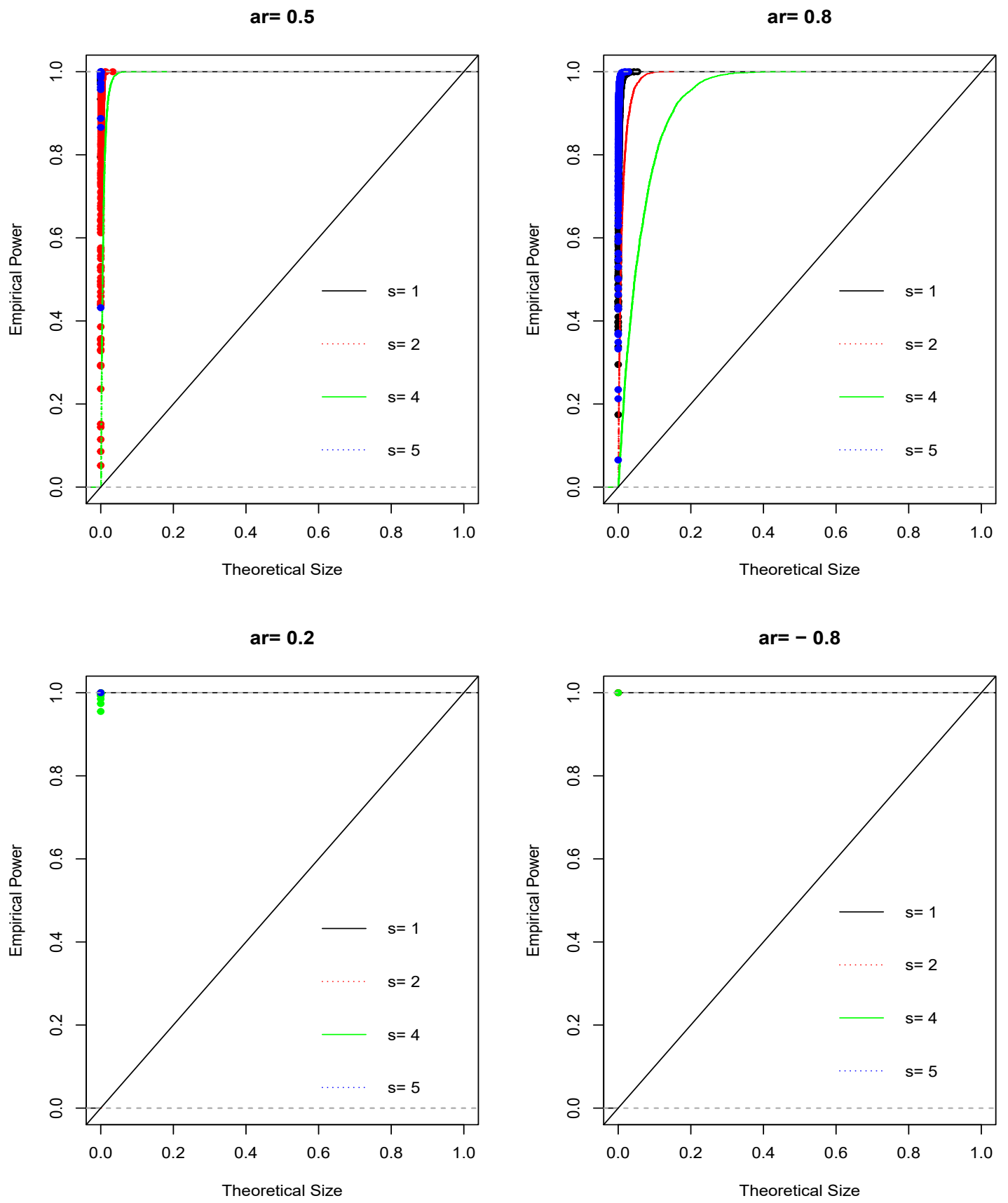

Figure 11: $Q_{n, m}^{T^{\prime}}\left(s, d_{G P H}\right), n=5000, m=n^{4}, X_{t}=\phi X_{t-1}+.005 t+\epsilon_{t}, \epsilon_{t} \stackrel{i . i . d}{\sim} \exp (1)-1$.

We can see from the above graphs that for all tested values of $\phi$ away from 1 the test yields high values of empirical power. 
effective at detecting the trend.

\subsection{Comparison}

In-line with the comments in Giraistis et al.(2006) we find that our statistic's are quite robust in detecting the presence of a linear trend. Given the limited amount of empirical power and size values generated from Giraistis et al.(2006), it is difficult to make conclusions on which statistic performs more robustly. However, under the null hypothesis it appears that our statistics do not experience the bias when $d \in(-.2, .2)$ and our statistics appear to yield the correct empirical size as $d$ gets close to .5 in comparision to the test from Giraistis et al.(2006).

\subsection{Conclusion}

The results from the $Q_{n, m}^{T^{\prime}}\left(s, d_{G P H}\right)$ test appear to indicate that as either $s$ or $n$ decreases, empirical size increases. Moreover, the $Q_{n, m}^{T^{\prime}}\left(s, d_{G P H}\right)$ appears to perform considerably well in regards to empirical size apart from $d \in(-.4,-.49)$. By switching between non-Gaussian or Gaussian innovations does not alter the findings above. Our findings demonstrate the existence of another statistical procedure to test for dependency against the presence of a trend. Compared to the existing time domain test, our statistic's yield a much simpler limiting distribution that can be obtained via light numerical computations which are much simpler than the required simulations involving stochastic integrals within Giraistis et al.(2006). We will now proceed to 
the part of this research where we apply the statistics developed within this research to real world data.

\section{Findings - Real World Example}

In this section we apply our statistical procedures to real world data sets in order to demonstrate their applicability and ability to produce desirable results outside of a synthetic context.

\subsection{First Procedure - SP500}

For the first data set we compare the $Q_{n, m=n \cdot 4}^{T}(1), Q_{n, m^{\cdot 5}}(2)$, VS, and LR tests using data from the returns from the Standard and Poor 500 Index (SP500). Previous research endeavours have assessed the squared returns of the SP500 during certain time periods for the presence of long memory. Such an example is Lobato (1997). The returns used here have been provided by Wharton Research Data Services (WRDS). The returns are generated from the period April 9th, 1973 to June 29th, 2018. The amount of observations is 10000 . The formula for the returns is given in the following: The returns we calculate with the following conventional formula

$$
r_{t}=\ln \left(P_{t}\right)-\ln \left(P_{t-1}\right),
$$

where $r_{t}$ is the return and $P_{t}$ is the price in period $t$.

We can see from Figure (12) the squared returns appear to not contain a trend 


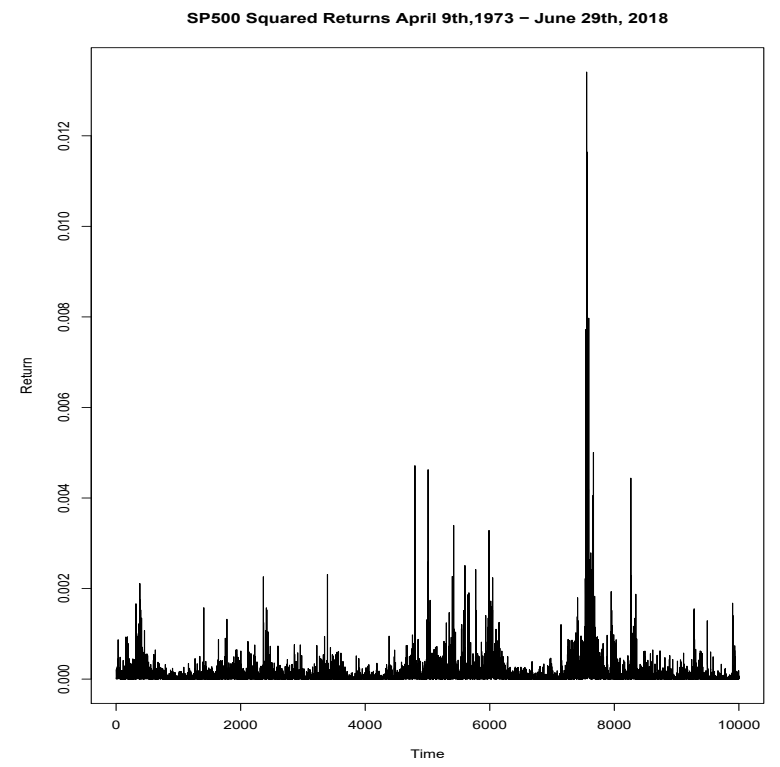

Figure 12: Time Plot of SP500 Squared Returns

that is either deterministic or stochastic. However, we can see that during certain periods, the series' volatility appears to change indicating that the series might be drawn from a process with conditional volatility. However, similar to researchers such as Lobato and Robinson (1998) and Giraistis et al.(2003) who found similar conditions with their data prior to implementing the procedure. Therefore, we proceed with our analysis of short memory versus long memory using this data.

We can see from graph on the left of Figure (13) that the ACF of the SP500 squared returns does not die down quickly. Moreover, by looking at the graph on the right of Figure (13) we can see that the periodogram seems to diverge at the origin. Therefore, the behaviour in the series depicted in both graphs alludes to the series potentially containing long memory. 

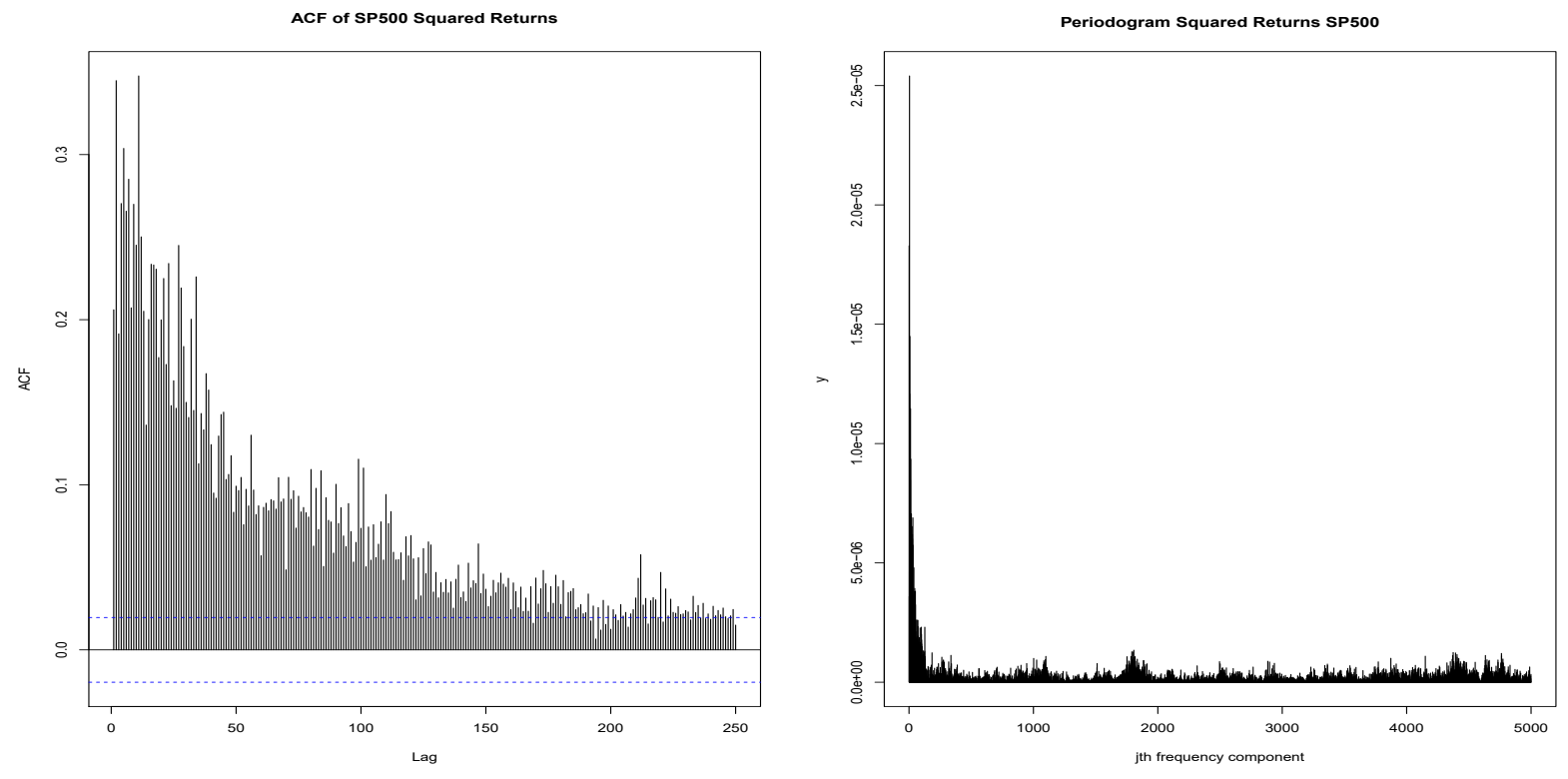

Figure 13: ACF and Periodogram of the Squared Returns from the SP500 Data

We then split the squared returns into ten and two blocks of sizes 1000 and 5000 respectively.

Indicated in Table (2) are the p-values for the four test statistics over each sample size. When evaluating the sample sizes of $n=1000$ we find that the test statistics perform almost analogously as they almost all reject the null each time at a theoretical size of .05. For the seventh block our proposed statistic fails to reject the null at all conventional nominal size levels. For the random taper we find that for the sixth and tenth block of size $n=1000$ we can see that the test fails to reject the null. This perhaps seems intuitive as in the comparison section of the first procedure it was observed that our proposed statistics yielded results that gave evidence of the test being more precise in identifying short memory with the null being true. Therefore, 
if indeed long memory is apparent here then we would expect our statistic to be less willing to reject the null hypothesis. Moreover, we also find that the VS statistic at the sixth block fails to reject the null at a theoretical size of .05 and the LR statistic slightly fails to reject the null three times. Inspecting each of the previously mentioned blocks' time, ACF, and periodogram, plots we fail to find conclusive evidence from a graphical perspective that alludes to the series being of short memory for any of these assessed blocks.

For the two blocks of sample size 5000 and entire array of 10000 we find that each of the test statistic obtain p-values of less than .01.

What one can infer from the results above is that each statistic rejects the null in almost each case. There are some slight discrepancies for which block each statistic claims as short memory. However, viewing the time, ACF, and periodogram plots of each block we find it hard to conclude which block is short memory as each one looks to deviate from this. Therefore, given the propensity of our statistics to reject the null in a manner similar to the VS and LR statistic, it appears our statistical procedures reacts very similar to the other previous statistical procedures in terms of distinguishing between long and short memory.

\subsection{Second Procedure - VIX}

For the secnod procedure we utilize the Chicago Board of Exchange's (CBOE's) SP500 Volatility Index (VIX) from the period starting January 2nd 1986 to Septem- 


\begin{tabular}{|l|l|l|l|l|l|l|}
\hline$\underline{\mathbf{n}}$ & $\underline{\text { Block} / \text { Stat }}$ & $\underline{\text { Cosine }}$ & $\underline{\text { Random }}$ & $\underline{\text { Untapered }}$ & $\underline{\text { LR }}$ & $\underline{\underline{\text { VS }}}$ \\
\hline 1000 & 1 & 0 & 0 & 0 & 0 & 0 \\
\hline 1000 & 2 & 0.000626 & 0 & 0 & 0.00444 & 0.00023 \\
\hline 1000 & 3 & 0 & 0.0001079101 & 0 & 0.00293 & 0.0001 \\
\hline 1000 & 4 & 0 & 0.0008175758 & 0 & 0.01334 & 0 \\
\hline 1000 & 5 & 0 & 0 & 0.00023984 & 0.03378 & 0.00032 \\
\hline 1000 & 6 & 0 & 0.08431577 & 0.00023603 & 0.00223 & 0.09489 \\
\hline 1000 & 7 & 0.348388 & 0 & 0 & 0 & 0 \\
\hline 1000 & 8 & 0 & 0 & 0 & 0 & 0 \\
\hline 1000 & 9 & 0 & 0 & 0 & 0.00146 & 0.00018 \\
\hline 1000 & 10 & 0.000625 & 0.02250928 & 0 & 0.01018 & 0.00345 \\
\hline
\end{tabular}

\begin{tabular}{|l|l|l|l|l|l|l|}
\hline 5000 & 1 & 0 & 0 & 0 & 0 & 0 \\
\hline 5000 & 2 & 0 & 0.0003677216 & 0 & 0 & 0.00023 \\
\hline
\end{tabular}

\begin{tabular}{|l|l|l|l|l|l|l|}
\hline 10000 & 1 & 0.002484 & 0.0008581886 & 0 & 0 & 0 \\
\hline
\end{tabular}

Table 2: P-value Table for Squared Returns of SP500 Data. 


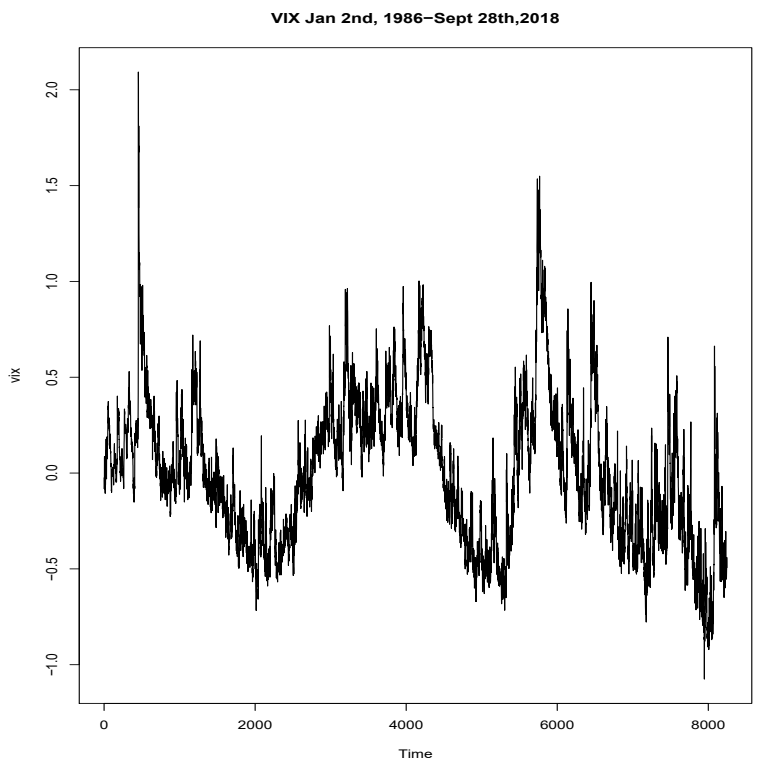

Figure 14: Time Plot De-meaned Log Transform of VIX Data.

ber 28th, 2018 within the four test statistics. The amount of observations is 8,254 . Apart form long memory the VIX has been assessed as well for structural breaks and other departures from non-stationarity. Similar to relevant literature, we take the $\log$ transformation and then de-mean the data. We view the ACF, periodogram, and time plots for the entire horizon and note that indeed the data looks to be from a zero mean process. However, determining stationarity looks to be an issue from a simple graphical perspective as the time plot looks to potentially depict the presence of a stochastic trend. Moreover, the ACF looks to experiences a large amount of spikes for many lag values and the periodogram accrues a huge spike at the origin while the $\mathrm{ACF}$ dies at a slow rate that can be hypothesized as being hyperbolic.

We divide the entire sample up into 2 and 8 blocks of sample sizes 4123 and 


\begin{tabular}{|l|l|l|l|l|}
\hline Sample Size & Block/Statistic & $\underline{\text { Cosine }}$ & $\underline{\text { ADF }}$ & $\underline{\underline{T}(\mathrm{~d})}$ \\
\hline 1031 & 1 & 0.09595 & 0.08 & $<.1$ \\
\hline 1031 & 2 & 0.91495 & 0.035 & $.05<\mathrm{p}<.1$ \\
\hline 1031 & 3 & 0.12997 & 0.01053 & $.05<\mathrm{p}<.1$ \\
\hline 1031 & 4 & 0.75078 & 0.01 & $>.1$ \\
\hline 1031 & 5 & 0.49951 & 0.4945 & $.05<\mathrm{p}<.1$ \\
\hline 1031 & 6 & 0.12471 & 0.581 & $.05<\mathrm{p}<.1$ \\
\hline 1031 & 7 & 0.02283 & 0.0491 & $>.1$ \\
\hline 1031 & 8 & 0.04306 & 0.01862 & $>.1$ \\
\hline
\end{tabular}

\begin{tabular}{|l|l|l|l|l|l|}
\hline 4123 & 1 & 0.00756 & 0.01349 & $<.05$ \\
\hline 4123 & 2 & 0.00291 & 0.01966 & $<.05$ \\
\hline
\end{tabular}

\begin{tabular}{|l|l|l|l|l|l|}
\hline 8246 & 1 & 0.01522 & 0.001 & $<.05$ \\
\hline
\end{tabular}

Table 3: P-value Table for the VIX Dataset 
1031, respectively. We run the data from each block through the $Q_{n, m}^{T^{\prime}}\left(s, d_{G P H}\right)$, and the test statistic from Giraistis et al.(2006) which we refer to as the $T(d)$ statistic. We also run each blocks data through the Augmented Dickey Fuller Test (ADF) as an auxiliary statistical procedure to assess for the presence of a trend. For the $T(d)$ statistic we use the quantiles given in Giraistis et al.(2006), as the limiting distribution is not readily available. We can see from Table (3) the $Q_{n, m}^{T^{\prime}}\left(s, d_{G P H}\right)$ test rejects the null the least. This seems to be intuitive as in our presented simulation studies it was depicted that the $Q_{n, m}^{T^{\prime}}\left(s, d_{G P H}\right)$ statistic was efficient in obtaining empirical size under the null. Given the results from the ADF test, it appears there is evidence that a stochastic trend is potentially present here for many of the blocks. With all of the above in mind, the $Q_{n, m}^{T^{\prime}}\left(s, d_{G P H}\right)$ statistic appears to behave in an intuitive manner given the graphical tools and the rejection decisions accrued for each of the assessed statistics.

With the above in mind we now proceed to the conclusion of the research.

\section{Conclusion}

Overall, we have found that our two statistical procedures yield results that allude to the existence of promising methods devised under the frequency domain that allow for the assessment of the second-order characteristics of a stationary processes. The tests we have presented for both procedures have demonstrated desirable common attributes stemming from both theoretical and applied standpoints. For instance, 
each of the proposed test statistics that utilized the cosine bell taper, yielded limiting distributions under the null that are quite obtainable with conventional statistical software via numerical integration. In respect to other popular tests which assess for analogous characteristics, the limiting distributions from our procedures are much simpler to generate than many other tests as they do not require stochastic integration. Furthermore, we have shown that our statistics are quite precise in obtaining the correct theoretical level under the null hypothesis for both procedures. Moreover, we have found that our test statistics behave in a stable manner for differing values of the window parameters $m$ and $s$ in a sense that by fixing $m$ the tests' empirical size and power increases as $s$ increases. In general, it appears that our statistics improve upon certain attributes of time domain tests for both procedures as they bear similar characteristics to previously existing periodogram-based tests. Furthermore, by implementing our statistics into real world data we have shown that the majority of our statistics demonstrate similar behaviour to other competing test statistics.

Our research has been performed with assumptions that allow for one to toggle between the frequency domain and time domain in regards to assessing the secondorder characteristics of a process. Therefore, our results potentially imply that under these assumptions one can construct procedures within the frequency domain utilizing the periodogram that allows for robust statistical inference to be performed. Given the asymptotic behaviour of the periodogram, the content within the frequency domain is easily accessible for many common forms of processes. We have demonstrated this by 
utilizing the tapered periodogram which is built upon the concept that for a strictly stationary process the normalized periodogram ordinates converge in distribution to a sum of weighted Chi-square random variables as Giraistis et al.(2012) show. Furthermore, it is well known that the periodogram itself has many methods which improve upon its estimation properties such as smoothing, windowing, tapering, and pre-whitening. Within this research we have merely endeavoured upon one of these methods by implementing one of the most popular and simplistic tapers in the form of the cosine bell taper. However, implementing popular and effective methods could potentially yield statistical procedures that are more robust. Therefore, given the vast array of techniques that are available to alter the periodogram in-conjunction with the probabilistic properties of the normalized periodogram ordinates, the possibility of creating further statistical procedures with desirable results seems to be considerable.

Furthermore, we suggest further exploring our statistics by finding an optimal set of window parameters from a theoretical perspective. For instance, similar flavoured efforts could be applied to random tapering as we have demonstrated the ability to apply a random weighting scheme in constructing a periodogram-based test. Therefore, we suggest that further research endeavours could be conducted in respect to random tapering. Overall, we hope that our efforts encourage future explorations to be conducted within the frequency domain in-regards to assessing the second order characteristics of a time series. 


\section{Appendix A}

\subsection{Additional Concepts}

Within this appendix we provide the theoretical results and concepts related to our research. The first concept we provide is in regards to stationarity.

A common characteristic a time series is usually assessed for is stationarity. Stationarity refers to the stable statistical properties of the series and is a vital assumption for using certain procedures and methods to derive inference. There are different degrees of stationarity such as strict stationarity and weak stationarity. For instance, the definition below gives a concise understanding of strict stationarity.

Definition 9.1 (Strict Stationarity). A time series $X_{t}$ is called strictly stationary if

$$
\left(X_{t_{1}}, X_{t_{2}}, \ldots, X_{t_{n}}\right) \stackrel{\mathbb{D}}{=}\left(X_{t_{1}+h}, X_{t_{2}+h}, \ldots, X_{t_{n}+h}\right) .
$$

For all time points $\left\{t_{1}, t_{2}, \ldots t_{n}\right\}$, where $n \in \mathbb{N}>0$, and for all $h \in \mathbb{Z}$

Strict stationarity assumes that the joint distribution of $\left\{X_{t_{1}}, X_{t_{2}}, \ldots, X_{t_{n}}\right\}$ is equivalent to that of the time-shifted set $\left\{X_{t_{1}+h}, X_{t_{2}+h}, \ldots, X_{t_{n}+h}\right\}$ as Shumway \& Stoffer (2011) state. It also implies that any multivariate joint distribution functions for subsets of the variables of $\left\{X_{t_{1}}, X_{t_{2}}, \ldots, X_{t_{n}}\right\}$ are equivalently distributed. For example, strict stationarity implies that $P\left(X_{t} \leq c\right)=P\left(X_{t+h} \leq c\right)$ for all integers $h$. In classical statistics, this is a common assumption when working with i.i.d data. However, in the context of time series processes this assumption is considered to be 
difficult to verify when placed into practice. However, many statistical endeavours are concerned with drawing inference only on the first few moments of the process. An assumption that allows for this is weak stationarity which states that the mean and variance of a process are constant over time and the covariance between neighbouring observations is a function only of the lag. The following definition which is from Shumway \& Stoffer (2011) gives a formal representation of this.

Definition 9.2 (Weak Stationarity). A time series $X_{t}$ is called weakly stationary if it is a finite variance process such that.

$$
\mathbb{E}\left(X_{t}\right)=\mu, \quad \operatorname{Cov}\left(X_{t}, X_{s}\right)=\gamma(t, s),
$$

where $\mu \in \mathbb{R}, \forall t$. Moreover, $\gamma$ is a function of $t$ or $s$ only through the difference in the $\operatorname{lag}|t-s|$.

It should be noted that underneath the existence of the second moment of $X_{t}$, weak stationarity is implied by strict stationarity. Weak stationarity is the more commonly assumed or assessed as it allows researchers to make assumptions on the first two moments and the covariance rather than the entire processes marginal and joint distributions. Moreover, weak stationarity also allows the results of the assessment of the correlation structure of the sample to be generalized towards the entire process. Generally speaking, the property of weak stationarity is referred simply as stationarity within time series literature. Therefore, from within this research we will follow suit and refer to weak stationarity as simply "stationarity". We would 
like to formally bring out the concept of the autocorrelation function (ACF) and autocovariance function (ACVF).

Definition 9.3 (Autocorrelation Function). Let $X_{t}$ be a stationary time series process. Then the ACF can be defined as:

$$
\operatorname{Corr}\left(X_{t}, X_{s}\right)=\rho(t, s)=\rho(h)=\frac{\gamma(h)}{\sigma_{X}^{2}},
$$

where $t-s=h$ Where $\gamma(h)=\operatorname{Cov}\left(X_{t}, X_{s}\right)$ is the ACVF. $\operatorname{Var}\left(X_{t}\right)=\sigma_{X}^{2}$.

With the above in mind we now speak of tapering. Tapering as Priestely (1981) states can be thought of for a sample $X_{t}, t=1, . . n$ as the multiplication of a function $h_{t}$ with an infinite realization of the process $X_{t}$. For instance, $h_{t}=$

$$
\begin{cases}1 & t=1, . ., n \\ 0 & \text { else }\end{cases}
$$

Since $h_{t}$ chances values sharply from 0 to 1 and $t=1$ and $t=n$, the DFT and the squared modulus of $h_{t}$ will experience variance spill over from a particular frequency component into other frequencies. Therefore, make it harder to pinpoint which components are actually contributing variance to the process. Since the DFT of a multiplication of two series is mathematically equivalent to the convolution of the DFT of each series individually.Therefore, the DFT of $h_{T} X_{t}$ will experience this leakage as well. As Priestely (1981) states this effect can be mitigated by choosing $h_{t}$ so that it introduces a certain function in place of $K_{n}(\theta)$ the Fejer kernel that reduces 
this leakage. Therefore, $h_{t}$ is chosen in a manner that allows for $h_{t}$ to change more smoothly from 0 to 1 and therefore, reduce leakage.

We now proceed to discuss the theoretical aspect of the graphical tools we use to assess our statistics' performance. Within this research we utilize P-value and Empirical Power plots for our simulation studies. These plots have been developed by Davidson \& Mackinnon (1997) and are based off of the well known concept that a continuous random variable's $\mathrm{CDF}$ follows a $U(0,1)$ distribution. For instance, this is can be demonstrated by the following definition which is taken from Casella and Berger (2001, Page 54, Theorem 2.1.10)

Theorem 9.4. (Probability Integral Transformation) Let $X$ be have a continuous cdf $F_{X}(x)$ and define the random variable $Y$ as $Y=F_{X}(x)$, then $Y$ is uniformly distributed on $(0,1)$, that is $P(Y \leq y)=y, 0<y<1$.

Proof. The reader is asked to turn to Casella and Berger (2001, Page 54, Theorem 2.1.10) for required referencing.

An example of the above is when we assume that $X$ is a continuous random variable with a CDF that is strictly increasing. Then the derivation of the transformation is as follows:

$$
F_{Y}(x)=P\left(F_{X}(X) \leq y\right)=P\left(X \leq F_{X}^{-1}(y)\right)=F_{X}\left(F_{X}^{-1}(y)\right)=y
$$

Where $y \in[0,1]$

Moreover, it can be shown that since $F_{X}(x) \sim U(0,1)$ then $1-F_{X}(x) \sim U(0,1)$. 
P-value plots provide a graphical method that allows for simplistic presentation of the properties of specification testing procedures in finite samples via Monte Carlo simulation as Davidson \& Mackinnon (1997) state. This tool allows for one to distinguish between a test statistic that systematically under-rejects, systematically overrejects, and rejects about the right proportion of the time as the authors state. Furthermore, the authors provide a theoretical explanation for their methods by letting $T$ be a test statistic and $T_{j}$ be the test statistic generated from the "jth" simulation where $j=1, . . N$. Furthermore, assume $p_{j}=p\left(T>T_{j}\right)$ to be the p-value of $T_{j}$.

$$
p_{j}=1-F_{T}\left(T_{j}\right) .
$$

The empirical distribution function (ECDF) of $p_{j}$ which is simply an estimate of the cdf of $p_{j}$ is defined as

$$
F^{*}\left(x_{i}\right)=\frac{1}{n} \sum_{j=1}^{n} I\left(p_{j} \leq x_{i}\right)
$$

At any point $x_{i} \in(0,1)$. Where

$$
I\left(p_{j} \leq x_{i}\right)= \begin{cases}1 & p_{j} \leq x_{i} \\ 0 & \text { else }\end{cases}
$$

Then the graph of $F^{*}\left(x_{i}\right)$ against $x_{i}$ is referred to as the P-value plot. As Davidson \& Mackinnon (1997) states if the distribution of $T\left(F_{T}\right)$ is correct then each of the $p_{j}$ should be distributed as $U(0,1)$. Therefore, the resulting graph should be close to the 45 degree line. When tests tend to plot above the straight line this indicates a procedure that systematically over-rejects the null at a given theoretical size where 
as a test that plots underneath the straight line indicates that the test systematically under-rejects the null at a given theoretical size. Under the alternate hypothesis one can evaluate a test's power by simulating under alternate conditions and performing the steps above for constructing a P-value plot. We refer to these plots simulated under the alternate as Empirical Power plots which are also presented in Davidson \& Mackinnon (1997) under the name "Power-Size" plots.

Another theorem which we implement continually within this research is the Continuous Mapping Theorem which we now state.

Theorem 9.5. (Continuous Mapping Theorem) Let $m \in \mathbb{N}>0$, and $q \in \mathbb{N}>0$, such that $q \leq m$. Assume $Y_{n}$ and $Y$ are $m$ dimensional real valued random vectors and $g: \mathbb{R}^{m} \rightarrow \mathbb{R}^{q}$ is a continuous function.

If

$$
Y_{n} \stackrel{\mathbb{P}}{\longrightarrow} Y,
$$

then

$$
g\left(Y_{n}\right) \stackrel{\mathbb{P}}{\longrightarrow} g(Y)
$$

We now speak of the the Lindenber Central Limit Theorem as it is related to the derivation of the limiting form of the components of the tapered periodogram.

Theorem 9.6. (Lindenberg Central Limit Theorem)

Let $X_{n, 1}, \ldots, X_{n, r_{n}}$ be a triangular array of Random Variables that are rowwise independent. Let $S_{n}=\sum_{k=1}^{r_{n}} X_{n, k}$ the following conditions be satsified: 


$$
\mathbb{E}\left(X_{n, k}\right)=0, \quad \sigma_{n, k}^{2}=\mathbb{E}\left(X_{n, k}^{2}\right)<\infty, \quad s_{n}^{2}=\sum_{k=1}^{r_{n}} \sigma_{n, k}^{2} .
$$

If

$$
\lim _{n \rightarrow \infty} \sum_{k=1}^{r_{n}} \frac{1}{s_{n}^{2}} \int_{\left|X_{n, k}\right| \geq \epsilon s_{n}} x^{2} d F_{X_{n, k}}(x)=0, \quad \forall \epsilon>0
$$

then

$$
\frac{S_{n}}{s_{n}} \stackrel{\mathbb{D}}{\longrightarrow} N(0,1)
$$

Proof. The proof can be found within Billingsley (1995).

As Billingsley (1995) states if (9.1) and (9.3) hold then a necessary condition for (9.2) to be true is the following:

$$
\max _{k \leq r_{n}} \frac{\sigma_{n, k}^{2}}{s_{n}^{2}} \rightarrow 0 .
$$

Which can be interpreted as the contribution of each $X_{n, k}$ 's variance becomes negligible when $n$ gets sufficiently large. It should be noted that if (9.4) holds then the Lindenberg Condition is necessary and sufficient for the CLT to hold. Another theorem that is worth mentioning is the Lyapounov's Condition extracted from Billingsley (1995). Suppose that $\left|X_{n, k}\right|^{2+\delta}$ is integratable for some positive $\delta$ and that Lyapounov's condition

$$
\lim _{n \rightarrow \infty} \sum_{k=1}^{r_{n}} \frac{1}{s_{n}^{2+\delta}} \mathbb{E}\left(\left|X_{n, k}\right|^{2+\delta}\right)=0
$$


holds, then the Lindenberg Condition holds as well. This is due to the fact that equation (9.2) is bounded above by

$$
\sum_{k=1}^{r_{n}} \frac{1}{s_{n}^{2}} \int_{\left|X_{n, k} \geq \epsilon s_{n}\right|} \frac{1}{\epsilon^{\delta} s_{n}^{\delta}}|x|^{2+\delta} d F_{X_{n_{k}}}=\leq \frac{1}{\delta} \sum_{k=1}^{r_{n}} \frac{1}{s_{n}^{2+\delta}} \mathbb{E}\left(\left|X_{n, k}\right|^{2+\delta}\right)
$$

The above leads to the following definiton which has been generated from Billingsley (1995, Theorem 27.3) states

Theorem 9.7. Let $X_{n, 1}, \ldots, X_{n, r_{n}}$ be a triangular array of Random Variables that are row-wise independent. Let $S_{n}=\sum_{k=1}^{r_{n}} X_{n, k}$ and the assumptions in (9.1) hold. Moreover, if (9.5) holds, then

$$
\frac{S_{n}}{s_{n}} \stackrel{\mathbb{D}}{\longrightarrow} N(0,1)
$$

In comparison to assumptions in the classical Central Limit Theorem, the Lyapounov and Lindenberg conditions trade in identical distribution conditions for stronger moment conditions. For instance, under the Lyapounov conditions one needs to verify that the moment of the order $2+\delta$ for some $\delta>0$, exists and that the growth of these moments is limited by the Lyapounov condition.

We now proceed to the next section of this appendix in which we demonstrate the theoretical concepts needed to derive the limiting distributions for our statistics.

\subsection{Theoretical Content}

Within this section we list required theoretical concepts and content required to derive the limiting forms of the statistics developed within our research and their asymptotic 
distributions.

If we define a new function as

$$
T_{n}(x)=\sum_{t=1}^{n} e^{i t x}=e^{i x} \frac{\left(1-e^{i n x}\right)}{\left(1-e^{i x}\right)}=\frac{\left(1-e^{i n x}\right)}{\left(e^{-i x}-1\right)}=\frac{1-\cos (n x)-i \sin (n x)}{\cos (x)-i \sin (x)-1},
$$

then

$$
\frac{1}{n} \lim _{n \rightarrow \infty} T_{n}\left(\frac{x}{n}\right)=\lim _{n \rightarrow \infty} \frac{1-\cos (x)-i \sin (x)}{\left(e^{-i x / n}-1\right) n}=\frac{i(1-\cos (x))+\sin (x)}{x},
$$

as

$$
\lim _{n \rightarrow \infty} \frac{1}{n\left(e^{-i x / n}-1\right)}=\frac{i}{x}
$$

Hence,

$$
\frac{1}{n} \lim _{n \rightarrow \infty} T_{n}\left(\frac{\theta-2 \pi j}{n}\right)=\frac{i\left(1-e^{i(\theta-2 \pi j)}\right)}{\theta-2 \pi j}=\frac{i\left(1-e^{i \theta}\right)}{\theta-2 \pi j} .
$$

Proposition 9.8. Let

$$
Y(\theta)=i\left(1-e^{i \theta}\right)=i(1-\cos (\theta))+\sin (\theta), \quad G(\theta)=\frac{1}{\theta(\theta+2 \pi)(\theta-2 \pi)}
$$

and

$$
P_{n}(\theta, j)=\sum_{t=1}^{n} \sin ^{2}\left(\frac{\pi t}{n}\right) \cos \left(\omega_{j} t\right) e^{i t \theta}, \quad Q_{n}(\theta, j)=\sum_{t=1}^{n} \sin ^{2}\left(\frac{\pi t}{n}\right) \sin \left(\omega_{j} t\right) e^{i t \theta}
$$

then

a.)

$$
\lim _{n \rightarrow \infty} \frac{1}{n} P_{n}\left(\frac{\theta}{n}, j\right)=-\pi^{2} Y(\theta) P(\theta, j),
$$


where

$$
P(\theta, j)=G(\theta+2 \pi j)+G(\theta-2 \pi j) .
$$

b.)

$$
\lim _{n \rightarrow \infty} \frac{1}{n} Q_{n}\left(\frac{\theta}{n}, j\right)=-i \pi^{2} Y(\theta) Q(\theta, j),
$$

where

$$
Q(\theta, j)=G(\theta-2 \pi j)-G(\theta+2 \pi j) .
$$

Proof. Let

$$
\begin{aligned}
P_{n}(\theta, j)=\sum_{t=1}^{n} \sin ^{2}\left(\frac{\pi t}{n}\right) \cos \left(\omega_{j} t\right) e^{i t \theta} & =\frac{1}{2} \sum_{t=1}^{n} \cos \left(\omega_{j} t\right) e^{i t \theta}-\frac{1}{2} \sum_{t=1}^{n} \cos \left(\frac{2 \pi t}{n}\right) \cos \left(\omega_{j} t\right) e^{i t \theta} \\
& =\frac{1}{2}\left(P_{n, 1}(\theta, j)-P_{n, 2}(\theta, j)\right)
\end{aligned}
$$

We note that

$$
\begin{aligned}
P_{n, 1}(\theta, j) & =\sum_{t=1}^{n} \cos \left(\omega_{j} t\right) e^{i t \theta} \\
& =\frac{1}{2} \sum_{t=1}^{n} e^{i t\left(\theta-\omega_{j}\right)}+\frac{1}{2} \sum_{t=1}^{n} e^{i t\left(\theta+\omega_{j}\right)} .
\end{aligned}
$$

Therefore,

$$
\begin{aligned}
P_{n, 1}\left(\frac{\theta}{n}, j\right) & =\left(\frac{1-\cos (\theta)-i \sin (\theta)}{2}\right)\left(\frac{1}{\cos ((\theta-2 \pi j) / n)-i \sin ((\theta-2 \pi j) / n)-1}+\right. \\
& \left.+\frac{1}{\cos ((\theta+2 \pi j) / n)-i \sin ((\theta+2 \pi j) / n)-1}\right) .
\end{aligned}
$$

By (9.6) we have

$$
\lim _{n \rightarrow \infty} \frac{1}{n} P_{n, 1}\left(\frac{\theta}{n}, j\right)=\left(\frac{i(1-\cos (\theta))+\sin (\theta)}{2}\right)\left(\frac{1}{\theta+2 \pi j}+\frac{1}{\theta-2 \pi j}\right) .
$$


Hence,

$$
\lim _{n \rightarrow \infty} \frac{1}{n} P_{n, 1}\left(\frac{\theta}{n}, j\right)=\frac{Y(\theta)}{2}\left(\frac{1}{\theta+2 \pi j}+\frac{1}{\theta-2 \pi j}\right)
$$

Furthermore,

$$
\begin{aligned}
P_{n, 2}(\theta, j) & =\sum_{t=1}^{n} \cos \left(\frac{2 \pi t}{n}\right) \cos \left(\omega_{j} t\right) e^{i t \theta} \\
& =\frac{1}{4} \sum_{t=1}^{n} e^{i t\left(\theta-\omega_{j}-2 \pi / n\right)}+\frac{1}{4} \sum_{t=1}^{n} e^{i t\left(\theta-\omega_{j}+2 \pi / n\right)}+\frac{1}{4} \sum_{t=1}^{n} e^{i t\left(\theta+\omega_{j}-2 \pi / n\right)}+ \\
& +\frac{1}{4} \sum_{t=1}^{n} e^{i t\left(\theta+\omega_{j}+2 \pi / n\right)} .
\end{aligned}
$$

Therefore, by applying (9.6) once more we have

$$
\lim _{n \rightarrow \infty} \frac{P_{n, 2}\left(\frac{\theta}{n}, j\right)}{n}=\frac{Y(\theta)}{4}\left(\frac{1}{\theta-2 \pi(j-1)}+\frac{1}{\theta-2 \pi(j+1)}+\frac{1}{\theta+2 \pi(j-1)}+\frac{1}{\theta+2 \pi(j+1)}\right) .
$$

Moreover, from the above results it is trivial to see that

$$
\begin{aligned}
& \lim _{n \rightarrow \infty} \frac{1}{n} P_{n}\left(\frac{\theta}{n}, j\right)=\lim _{n \rightarrow \infty} \frac{1}{2 n}\left(P_{n, 1}\left(\frac{\theta}{n}, j\right)-P_{n, 2}\left(\frac{\theta}{n}, j\right)\right)= \\
& =\frac{Y(\theta)}{8}\left(\frac{2}{\theta+2 \pi j}+\frac{2}{\theta-2 \pi j}-\right. \\
& \left.-\frac{1}{\theta-2 \pi(j-1)}-\frac{1}{\theta-2 \pi(j+1)}-\frac{1}{\theta+2 \pi(j-1)}-\frac{1}{\theta+2 \pi(j+1)}\right) .
\end{aligned}
$$


We note that

$$
\begin{aligned}
& \frac{2}{\theta+2 \pi j}-\frac{1}{\theta+2 \pi(j+1)}-\frac{1}{\theta+2 \pi(j-1)}= \\
= & \frac{2}{\theta+2 \pi j}-\frac{\theta+2 \pi(j+1)+\theta+2 \pi(j-1)}{(\theta+2 \pi(j+1))(\theta+2 \pi(j-1))} \\
= & \frac{2}{\theta+2 \pi j}-\frac{2 \theta+4 \pi j}{(\theta+2 \pi(j+1))(\theta+2 \pi(j-1))} \\
= & 2\left(\frac{(\theta+2 \pi(j+1))(\theta+2 \pi(j-1))-(\theta+2 \pi j)(\theta+2 \pi j)}{(\theta+2 \pi j)(\theta+2 \pi(j+1))(\theta+2 \pi(j-1))}\right) \\
= & 2\left(\frac{(\theta+2 \pi j))^{2}-4 \pi^{2}-(\theta+2 \pi j)^{2}}{(\theta+2 \pi j)(\theta+2 \pi(j+1))(\theta+2 \pi(j-1))}\right) \\
= & -8 \pi^{2} G(\theta+2 \pi j) .
\end{aligned}
$$

Hence,

$$
\frac{2}{\theta+2 \pi j}-\frac{1}{\theta+2 \pi(j+1)}-\frac{1}{\theta+2 \pi(j-1)}=-8 \pi^{2} G(\theta+2 \pi j) .
$$

Similarly, using the above with $j=-j$, one can solve for

$$
\frac{2}{\theta-2 \pi j}-\frac{1}{\theta-2 \pi(j+1)}-\frac{1}{\theta-2 \pi(j-1)}=-8 \pi^{2} G(\theta-2 \pi j) .
$$

Hence, rearranging the above results we get

$$
\lim _{n \rightarrow \infty} \frac{1}{n} P_{n}\left(\frac{\theta}{n}, j\right)=-\pi^{2} Y(\theta)(G(\theta+2 \pi j)+G(\theta-2 \pi j))=-\pi^{2} Y(\theta) P(\theta, j) .
$$

Part b.) We note initially that

$$
\begin{aligned}
Q_{n}(\theta, j) & =\sum_{t=1}^{n} \sin ^{2}\left(\frac{\pi t}{n}\right) \sin \left(\omega_{j} t\right) e^{i t \theta}=\frac{1}{2} \sum_{t=1}^{n} \sin \left(\omega_{j} t\right) e^{i t \theta}-\frac{1}{2} \sum_{t=1}^{n} \cos \left(\frac{2 \pi t}{n}\right) \sin \left(\omega_{j} t\right) e^{i t \theta} \\
= & \frac{1}{2}\left(Q_{n, 1}(\theta, j)-Q_{n, 2}(\theta, j)\right) .
\end{aligned}
$$


Where,

$$
\begin{aligned}
Q_{n, 1}(\theta, j) & =\sum_{t=1}^{n} \sin \left(\omega_{j} t\right) e^{i t \theta} \\
& =\frac{i}{2} \sum_{t=1}^{n} e^{i t\left(\theta-\omega_{j}\right)}-\frac{i}{2} \sum_{t=1}^{n} e^{i t\left(\theta+\omega_{j}\right)} .
\end{aligned}
$$

Moreover,

$$
\begin{aligned}
Q_{n, 1}\left(\frac{\theta}{n}, j\right) & =\left(\frac{i(1-\cos (\theta))+\sin (\theta)}{2}\right)\left(\frac{1}{\cos ((\theta-2 \pi j) / n)-i \sin ((\theta-2 \pi j) / n)-1}-\right. \\
& \left.-\frac{1}{\cos ((\theta+2 \pi j) / n)-i \sin ((\theta+2 \pi j) / n)-1}\right) .
\end{aligned}
$$

Furthermore, by (9.6) we have

$$
\lim _{n \rightarrow \infty} \frac{1}{n} Q_{n, 1}\left(\frac{\theta}{n}, j\right)=\left(\frac{i Y(\theta)}{2}\right)\left(\frac{1}{\theta-2 \pi j}-\frac{1}{\theta+2 \pi j}\right) .
$$

Similar to the computations above we can see

$$
\begin{aligned}
Q_{n, 2}(\theta, j) & =\sum_{t=1}^{n} \cos \left(\frac{2 \pi t}{n}\right) \sin \left(\omega_{j} t\right) e^{i t \theta} \\
& =\frac{i}{4} \sum_{t=1}^{n} e^{i t\left(\theta-\omega_{j}-2 \pi / n\right)}+\frac{i}{4} \sum_{t=1}^{n} e^{i t\left(\theta-\omega_{j}+2 \pi / n\right)}-\frac{i}{4} \sum_{t=1}^{n} e^{i t\left(\theta+\omega_{j}-2 \pi / n\right)}- \\
& -\frac{i}{4} \sum_{t=1}^{n} e^{i t\left(\theta+\omega_{j}+2 \pi / n\right)} .
\end{aligned}
$$

Moreover, by applying (9.6) to the above we have

$$
\begin{aligned}
& \lim _{n \rightarrow \infty} \frac{1}{n} Q_{n, 2}\left(\frac{\theta}{n}, j\right)= \\
& =\left(\frac{i Y(\theta)}{4}\right)\left(\frac{1}{\theta-2 \pi(j-1)}+\frac{1}{\theta-2 \pi(j+1)}-\frac{1}{\theta+2 \pi(j-1)}-\frac{1}{\theta+2 \pi(j+1)}\right) .
\end{aligned}
$$


Hence,

$$
\begin{aligned}
& \lim _{n \rightarrow \infty} \frac{1}{2 n} Q_{n}\left(\frac{\theta}{n}, j\right)=\lim _{n \rightarrow \infty} \frac{1}{n}\left(Q_{n, 1}\left(\frac{\theta}{n}, j\right)-Q_{n, 2}\left(\frac{\theta}{n}, j\right)\right) \\
& =-i \pi^{2} Y(\theta)(G(\theta-2 \pi j)-G(\theta+2 \pi j)) \\
& =-i \pi^{2} Y(\theta) Q(\theta, j)
\end{aligned}
$$

We also note that

$$
\begin{aligned}
Y(\theta) \overline{Y(\theta)} & \left.=(1-\cos (\theta))^{2}+\sin ^{2}(\theta)\right) \\
& =1-2 \cos (\theta)+\cos ^{2}(\theta)+\sin ^{2}(\theta) \\
& =2(1-\cos (\theta)) \\
& =4 \sin ^{2}\left(\frac{\theta}{2}\right)
\end{aligned}
$$

$$
Y(\theta) \overline{Y(\theta)}=4 \sin ^{2}\left(\frac{\theta}{2}\right)
$$

Proposition 9.9. Let $d \in(-.5, .5)$ and $r, u=0,1,2$. Furthermore, for simplicity let

$$
\begin{aligned}
& P_{n}(\theta, j)=P_{n, 0}(\theta, j), \text { and } Q_{n}(\theta, j)=Q_{n, 0}(\theta, j), \text { then } \\
& \lim _{n \rightarrow \infty} \int_{-n \pi}^{n \pi} \frac{\theta^{-2 d}}{n^{2}} P_{n, r}\left(\frac{\theta}{n}, j\right) \overline{P_{n, u}\left(\frac{\theta}{n}, k\right)} d \theta=\int_{-\infty}^{\infty} \lim _{n \rightarrow \infty} \frac{\theta^{-2 d}}{n^{2}} P_{n, r}\left(\frac{\theta}{n}, j\right) \overline{P_{n, u}\left(\frac{\theta}{n}, k\right)} d \theta \\
& \lim _{n \rightarrow \infty} \int_{-n \pi}^{n \pi} \frac{\theta^{-2 d}}{n^{2}} P_{n, r}\left(\frac{\theta}{n}, j\right) \overline{Q_{n, u}\left(\frac{\theta}{n}, k\right)} d \theta=\int_{-\infty}^{\infty} \lim _{n \rightarrow \infty} \frac{\theta^{-2 d}}{n^{2}} P_{n, r}\left(\frac{\theta}{n}, j\right) \overline{Q_{n, u}\left(\frac{\theta}{n}, k\right)} d \theta \\
& \lim _{n \rightarrow \infty} \int_{-n \pi}^{n \pi} \frac{\theta^{-2 d}}{n^{2}} Q_{n, r}\left(\frac{\theta}{n}, j\right) \overline{Q_{n, u}\left(\frac{\theta}{n}, k\right)} d \theta=\int_{-\infty}^{\infty} \lim _{n \rightarrow \infty} \frac{\theta^{-2 d}}{n^{2}} Q_{n, r}\left(\frac{\theta}{n}, j\right) \overline{Q_{n, u}\left(\frac{\theta}{n}, k\right)} d \theta .
\end{aligned}
$$


Proof. We note that it can be extracted from Hurvich \& Beltrao (1993, Theorem 3 and Theorem 8) we have for fixed integers $j, k \in(-n / 2, n / 2)$

$$
\lim _{n \rightarrow \infty} \int_{-n \pi}^{n \pi} \frac{1}{n^{2}} \frac{\sin ^{2}\left(\frac{\theta}{2}\right)}{\sin \left(\frac{\theta-2 \pi j}{2 n}\right) \sin \left(\frac{\theta-2 \pi k}{2 n}\right)}|\theta|^{-2 d} d \theta=\int_{-\infty}^{\infty} \frac{4 \sin ^{2}\left(\frac{\theta}{2}\right)}{(\theta-2 \pi j)(\theta-2 \pi k)}|\theta|^{-2 d} d \theta .
$$

Moreover, we also note that

$$
\sum_{t=1}^{n} e^{i t x}=\frac{e^{i x}\left(e^{i n x}-1\right)}{\left(e^{i x}-1\right)}=\frac{e^{i x} e^{i n x / 2}\left(e^{i n x / 2}-e^{-i n x / 2}\right)}{e^{i x / 2}\left(e^{i x / 2}-e^{-i x / 2}\right)}=\frac{e^{i x(n+1) / 2} \sin (n x / 2)}{\sin (x / 2)} .
$$

Therefore, the functions within the following class

$$
\left\{\frac{\theta^{-2 d}}{n^{2}} P_{n, r}\left(\frac{\theta}{n}, j\right) \overline{P_{n, u}\left(\frac{\theta}{n}, k\right)}, \frac{\theta^{-2 d}}{n^{2}} P_{n, r}\left(\frac{\theta}{n}, j\right) \overline{Q_{n, u}\left(\frac{\theta}{n}, k\right)}, \frac{\theta^{-2 d}}{n^{2}} Q_{n, r}\left(\frac{\theta}{n}, j\right) \overline{Q_{n, u}\left(\frac{\theta}{n}, k\right)}\right\},
$$

are all linear combinations (in $\theta$ ) of

$$
\frac{1}{n^{2}} \frac{\sin ^{2}\left(\frac{\theta}{2}\right)}{\sin \left(\frac{\theta-2 \pi j}{2 n}\right) \sin \left(\frac{\theta-2 \pi k}{2 n}\right)}|\theta|^{-2 d} d \theta .
$$

Hence, the limit can be transferred underneath the integral operator when integrating any of the functions in the above class.

Proposition 9.10. Let $X_{t}$ be a zero mean stationary process with spectral density of the form of (2.6) where $d \in(-.5, .5)$, then

$$
\text { a.) }
$$$$
\lim _{n \rightarrow \infty} \operatorname{Cov}\left(\frac{A_{T}\left(\omega_{j}\right)}{\sqrt{f\left(\omega_{j}\right)}}, \frac{A_{T}\left(\omega_{k}\right)}{\sqrt{f\left(\omega_{k}\right)}}\right)=\frac{16 \pi^{3}}{3} \int_{-\infty}^{\infty} \sin ^{2}\left(\frac{\theta}{2}\right) P(\theta, j) P(\theta, k)\left(\frac{\theta}{\sqrt{2 \pi j k}}\right)^{-2 d} d \theta .
$$$$
\text { b.) }
$$$$
\lim _{n \rightarrow \infty} \operatorname{Cov}\left(\frac{B_{T}\left(\omega_{j}\right)}{\sqrt{f\left(\omega_{j}\right)}}, \frac{B_{T}\left(\omega_{k}\right)}{\sqrt{f\left(\omega_{k}\right)}}\right)=\frac{16 \pi^{3}}{3} \int_{-\infty}^{\infty} \sin ^{2}\left(\frac{\theta}{2}\right) Q(\theta, j) Q(\theta, k)\left(\frac{\theta}{2 \pi \sqrt{j k}}\right)^{-2 d} d \theta .
$$ 
c.)

$$
\lim _{n \rightarrow \infty} \operatorname{Cov}\left(\frac{A_{T}\left(\omega_{j}\right)}{\sqrt{f\left(\omega_{j}\right)}}, \frac{B_{T}\left(\omega_{k}\right)}{\sqrt{f\left(\omega_{k}\right)}}\right)=0 .
$$

Proof. We note

$$
\begin{aligned}
\left(A_{T}\left(\omega_{j}\right) A_{T}\left(\omega_{k}\right)\right) & =\frac{4}{3 \pi n} \sum_{t=1}^{n} h_{t} X_{t} \cos \left(\omega_{j} t\right) \sum_{s=1}^{n} h_{s} X_{s} \cos \left(\omega_{k} s\right) \\
& =\frac{4}{3 \pi n} \int_{-\pi}^{\pi} \int_{-\pi}^{\pi} \sum_{t=1}^{n} h_{t} e^{i t \theta} \cos \left(\omega_{j} t\right) \sum_{s=1}^{n} h_{s} e^{i s \theta^{\prime}} \cos \left(\omega_{k} s\right) d Z(\theta) d Z\left(\theta^{\prime}\right) \\
& =\frac{4}{3 \pi n} \int_{-\pi}^{\pi} \int_{-\pi}^{\pi} \sum_{t=1}^{n} h_{t} e^{i t \theta} \cos \left(\omega_{j} t\right) \sum_{s=1}^{n} h_{s} e^{-i s \theta^{\prime}} \cos \left(\omega_{k} s\right) d Z(\theta) d Z\left(-\theta^{\prime}\right) \\
& =\frac{4}{3 \pi n} \int_{-\pi}^{\pi} \int_{-\pi}^{\pi} \sum_{t=1}^{n} h_{t} e^{i t \theta} \cos \left(\omega_{j} t\right) \sum_{s=1}^{n} h_{s} e^{-i s \theta^{\prime}} \cos \left(\omega_{k} s\right) d Z(\theta) \overline{d Z\left(\theta^{\prime}\right)} \\
& =\frac{4}{3 \pi n} \int_{-\pi}^{\pi} \int_{-\pi}^{\pi} P_{n}(\theta, j) \overline{P_{n}\left(\theta^{\prime}, k\right)} d Z(\theta) \overline{d Z\left(\theta^{\prime}\right)}
\end{aligned}
$$

Hence,

$$
\begin{aligned}
\operatorname{Cov}\left(\frac{A_{T}\left(\omega_{j}\right)}{\sqrt{f\left(\omega_{j}\right)}}, \frac{A_{T}\left(\omega_{k}\right)}{\sqrt{f\left(\omega_{k}\right)}}\right) & =\mathbb{E}\left(\frac{A_{T}\left(\omega_{j}\right)}{\sqrt{f\left(\omega_{j}\right)}} \frac{A_{T}\left(\omega_{k}\right)}{\sqrt{f\left(\omega_{k}\right)}}\right) \\
& =\frac{1}{\sqrt{f\left(\omega_{j}\right) f\left(\omega_{k}\right)}} \frac{4}{3 \pi n} \int_{-\pi}^{\pi} \int_{-\pi}^{\pi} P_{n}(\theta, j) \overline{P_{n}\left(\theta^{\prime}, k\right)} \mathbb{E}\left(d Z(\theta) \overline{d Z\left(\theta^{\prime}\right)}\right) \\
& =\frac{1}{\sqrt{f\left(\omega_{j}\right) f\left(\omega_{k}\right)}} \frac{4}{3 \pi n} \int_{-\pi}^{\pi} \int_{-\pi}^{\pi} P_{n}(\theta, j) \overline{P_{n}(\theta, k)} \mathbb{E}\left(|d Z(\theta)|^{2}\right) \\
& =\frac{4}{3 \pi n} \int_{-\pi}^{\pi} P_{n}(\theta, j) \overline{P_{n}(\theta, k)} f(\theta) d \theta \\
& \text { Let } \lambda=\frac{\theta}{n} \rightarrow d \lambda=\frac{d \theta}{n}, \\
& =\frac{4}{3 \pi n^{2}} \int_{-n \pi}^{n \pi} P_{n}(\theta / n, j) \overline{P_{n}(\theta / n, k)} \frac{f(\theta / n)}{\sqrt{f\left(\omega_{j}\right) f\left(\omega_{k}\right)}} d \theta \\
& \sim \frac{4}{3 \pi n^{2}} \int_{-n \pi}^{n \pi} P_{n}(\theta / n, j) \overline{P_{n}(\theta / n, k)}\left(\frac{\theta}{\sqrt{2 \pi j k}}\right)^{-2 d} d \theta .
\end{aligned}
$$


Moreover, by (9.11) and (9.12) we have

$$
\begin{aligned}
\lim _{n \rightarrow \infty} \frac{1}{n^{2}} P_{n}(\theta / n, j) \overline{P_{n}(\theta / n, k)} & =\pi^{4} Y(\theta) \overline{Y(\theta)} P(\theta, j) P(\theta, k) \\
& =4 \pi^{4} \sin ^{2}\left(\frac{\theta}{2}\right) P(\theta, j) P(\theta, k)
\end{aligned}
$$

Hence, by Proposition (9.9) we have

$$
\lim _{n \rightarrow \infty} \mathbb{E}\left(\frac{A_{T}\left(\omega_{j}\right)}{\sqrt{f\left(\omega_{j}\right)}} \frac{A_{T}\left(\omega_{k}\right)}{\sqrt{f\left(\omega_{k}\right)}}\right)=\frac{16 \pi^{3}}{3} \int_{-\infty}^{\infty} \sin ^{2}\left(\frac{\theta}{2}\right) P(\theta, j) P(\theta, k)\left(\frac{\theta}{\sqrt{2 \pi j k}}\right)^{-2 d} d \theta
$$

Part b.)

Similar to the above in Part a.) we can find the limiting value of the covariance of the sine components of the tapered periodogram by using the Spectral Representation Theorem and Proposition (9.9). Therefore,

$$
\begin{aligned}
& \mathbb{E}\left(\frac{B_{T}\left(\omega_{j}\right)}{\sqrt{f\left(\omega_{j}\right)}} \frac{B_{T}\left(\omega_{k}\right)}{\sqrt{f\left(\omega_{k}\right)}}\right)=\mathbb{E}\left(\frac{1}{\sqrt{f\left(\omega_{j}\right) f\left(\omega_{k}\right)}} \frac{4}{3 \pi n} \sum_{t=1}^{n} h_{t} X_{t} \sin \left(\omega_{j} t\right) \sum_{s=1}^{n} h_{s} X_{s} \sin \left(\omega_{k} s\right)\right) \\
& =\frac{4}{3 \pi n} \int_{-\pi}^{\pi} \int_{-\pi}^{\pi} \sum_{t=1}^{n} h_{t} e^{i t \theta} \sin \left(\omega_{j} t\right) \sum_{s=1}^{n} h_{s} e^{i s \theta^{\prime}} \sin \left(\omega_{k} s\right) \mathbb{E}\left(d Z(\theta) \overline{d Z\left(\theta^{\prime}\right)}\right) \\
& =\frac{4}{3 \pi n} \int_{-\pi}^{\pi} \sin ^{2}\left(\frac{\theta}{2}\right) Q_{n}(\theta, j) \overline{Q_{n}(\theta, k)} \frac{f(\theta)}{\sqrt{f\left(\omega_{j}\right) f\left(\omega_{k}\right)}} d \theta \\
& =\frac{4}{3 \pi n^{2}} \int_{-n \pi}^{n \pi} \sin ^{2}\left(\frac{\theta}{2}\right) Q_{n}(\theta / n, j) \overline{Q_{n}(\theta / n, k)} \frac{f(\theta / n)}{\sqrt{f\left(\omega_{j}\right) f\left(\omega_{k}\right)}} d \theta \\
& \sim \frac{4}{3 \pi n^{2}} \int_{-n \pi}^{n \pi} \sin ^{2}\left(\frac{\theta}{2}\right) Q_{n}(\theta / n, j) \overline{Q_{n}(\theta / n, k)}\left(\frac{\theta}{2 \pi \sqrt{j k}}\right)^{-2 d} d \theta \\
& \longrightarrow \frac{16 \pi^{3}}{3} \int_{-\infty}^{\infty} \sin ^{2}\left(\frac{\theta}{2}\right) Q(\theta, j) Q(\theta, k)\left(\frac{\theta}{2 \pi \sqrt{j k}}\right)^{-2 d} d \theta
\end{aligned}
$$

as $n \rightarrow \infty$. 
Part c.) Analogous to the computations above we have

$$
\begin{aligned}
& \mathbb{E}\left(\frac{A_{T}\left(\omega_{j}\right)}{\sqrt{f\left(\omega_{j}\right)}} \frac{B_{T}\left(\omega_{k}\right)}{\sqrt{f\left(\omega_{k}\right)}}\right)=\mathbb{E}\left(\frac{1}{\sqrt{f\left(\omega_{j}\right) f\left(\omega_{k}\right)}} \frac{4}{3 \pi n} \sum_{t=1}^{n} h_{t} X_{t} \cos \left(\omega_{j} t\right) \sum_{s=1}^{n} h_{s} X_{s} \sin \left(\omega_{k} s\right)\right) \\
& =\frac{4}{3 \pi n} \int_{-\pi}^{\pi} \int_{-\pi}^{\pi} \sum_{t=1}^{n} h_{t} e^{i t \theta} \sin \left(\omega_{j} t\right) \sum_{s=1}^{n} h_{s} e^{i s \theta^{\prime}} \sin \left(\omega_{k} s\right) \mathbb{E}\left(d Z(\theta) \overline{d Z\left(\theta^{\prime}\right)}\right) \\
& =\frac{4}{3 \pi n} \int_{-\pi}^{\pi} \sin ^{2}\left(\frac{\theta}{2}\right) P_{n}(\theta, j) \overline{Q_{n}(\theta, k)} \frac{f(\theta)}{\sqrt{f\left(\omega_{j}\right) f\left(\omega_{k}\right)}} d \theta \\
& =\frac{4}{3 \pi n^{2}} \int_{-n \pi}^{n \pi} \sin ^{2}\left(\frac{\theta}{2}\right) P_{n}(\theta, j) \overline{Q_{n}(\theta, k)} \frac{f(\theta / n)}{\sqrt{f\left(\omega_{j}\right) f\left(\omega_{k}\right)}} d \theta \\
& \sim \frac{4}{3 \pi n^{2}} \int_{-n \pi}^{n \pi} \sin ^{2}\left(\frac{\theta}{2}\right) P_{n}(\theta, j) \overline{Q_{n}(\theta, k)}\left(\frac{\theta}{2 \pi \sqrt{j k}}\right)^{-2 d} d \theta \\
& \longrightarrow \frac{-16 \pi^{3} i}{3} \int_{-\infty}^{\infty} \sin ^{2}\left(\frac{\theta}{2}\right) P(\theta, j) Q(\theta, k)\left(\frac{\theta}{2 \pi \sqrt{j k}}\right)^{-2 d} d \theta \\
& =0 \text {, }
\end{aligned}
$$

by the results of Proposition (9.9) and Lemma (9.16) as $n \rightarrow \infty$. We note that the underlying reason for the limiting integral to be equal to zero is due to the fact that

$$
\sin ^{2}\left(\frac{\theta}{2}\right) P(\theta, j) Q(\theta, k)\left(\frac{\theta}{2 \pi \sqrt{j k}}\right)^{-2 d},
$$

is odd in $\theta$.

Proposition 9.11. Let $X_{t}$ be of the form of Proposition (2.3) with spectral density $f$ of the form of (2.6) where $d \in(-.5, .5)$. Let

$$
L_{j, k}(d)=\frac{2}{\pi} \int_{-\infty}^{\infty} \sin ^{2}\left(\frac{\theta}{2}\right)\left(\frac{1}{(\theta-2 \pi j)} \frac{1}{(\theta-2 \pi k)}\right)\left(\frac{\theta}{2 \pi \sqrt{j k}}\right)^{-2 d} d \theta
$$

and

$$
R_{j, k}(d)=\frac{2}{\pi} \int_{-\infty}^{\infty} \sin ^{2}\left(\frac{\theta}{2}\right)\left(\frac{1}{(\theta+2 \pi j)} \frac{1}{(2 \pi k-\theta)}\right)\left(\frac{\theta}{2 \pi \sqrt{j k}}\right)^{-2 d} d \theta
$$


then

a.)

$$
\lim _{n \rightarrow \infty} \operatorname{Cov}\left(\frac{A\left(\omega_{j}\right)}{\sqrt{f\left(\omega_{j}\right)}}, \frac{A\left(\omega_{k}\right)}{\sqrt{f\left(\omega_{k}\right)}}\right)=L_{j, k}(d)-R_{j, k}(d) .
$$

b.)

$$
\lim _{n \rightarrow \infty} \operatorname{Cov}\left(\frac{B\left(\omega_{j}\right)}{\sqrt{f\left(\omega_{j}\right)}}, \frac{B\left(\omega_{k}\right)}{\sqrt{f\left(\omega_{k}\right)}}\right)=L_{j, k}(d)+R_{j, k}(d) .
$$

c.)

$$
\lim _{n \rightarrow \infty} \operatorname{Cov}\left(\frac{A\left(\omega_{j}\right)}{\sqrt{f\left(\omega_{j}\right)}}, \frac{B\left(\omega_{k}\right)}{\sqrt{f\left(\omega_{k}\right)}}\right)=0 .
$$

d.)

$$
\lim _{n \rightarrow \infty} \operatorname{Cov}\left(\frac{D\left(\omega_{j}\right)}{\sqrt{f\left(\omega_{j}\right)}}, \frac{D\left(\omega_{k}\right)}{\sqrt{f\left(\omega_{k}\right)}}\right)=L_{j, k}(d) .
$$

Proof. We note that we have included the derivation for this proof as Hurvich \& Beltrao (1993) omits the direct derivations of the desired results. Therefore, we felt it would be beneficial for demonstrative purposes to include them within this research 
by utilizing Proposition (9.9).

$$
\begin{aligned}
& \mathbb{E}\left(\frac{A\left(\omega_{j}\right)}{\sqrt{f\left(\omega_{j}\right)}} \frac{A\left(\omega_{k}\right)}{\sqrt{f\left(\omega_{k}\right)}}\right)=\mathbb{E}\left(\frac{1}{\sqrt{f\left(\omega_{j}\right) f\left(\omega_{k}\right)}} \frac{1}{2 \pi n} \sum_{t=1}^{n} X_{t} \cos \left(\omega_{j} t\right) \sum_{s=1}^{n} X_{s} \cos \left(\omega_{k} s\right)\right) \\
& =\frac{1}{2 \pi n} \int_{-\pi}^{\pi} \int_{-\pi}^{\pi} \sum_{t=1}^{n} e^{i t \theta} \cos \left(\omega_{j} t\right) \sum_{s=1}^{n} e^{i s \theta^{\prime}} \cos \left(\omega_{k} s\right) \mathbb{E}\left(d Z(\theta) \overline{d Z\left(\theta^{\prime}\right)}\right) \\
& =\frac{1}{2 \pi n} \int_{-\pi}^{\pi} \sin ^{2}\left(\frac{\theta}{2}\right) P_{n, 1}(\theta, j) \overline{P_{n, 1}(\theta, k)} \frac{f(\theta)}{\sqrt{f\left(\omega_{j}\right) f\left(\omega_{k}\right)}} d \theta \\
& =\frac{1}{2 \pi n^{2}} \int_{-n \pi}^{n \pi} \sin ^{2}\left(\frac{\theta}{2}\right) P_{n, 1}(\theta, j) \overline{P_{n, 1}(\theta, k)} \frac{f(\theta / n)}{\sqrt{f\left(\omega_{j}\right) f\left(\omega_{k}\right)}} d \theta \\
& \sim \frac{1}{2 \pi n^{2}} \int_{-n \pi}^{n \pi} \sin ^{2}\left(\frac{\theta}{2}\right) P_{n, 1}(\theta, j) \overline{P_{n, 1}(\theta, k)}\left(\frac{\theta}{2 \pi \sqrt{j k}}\right)^{-2 d} d \theta \\
& \longrightarrow \frac{1}{2 \pi} \int_{-\infty}^{\infty} \sin ^{2}\left(\frac{\theta}{2}\right)\left(\frac{1}{\theta-2 \pi j}+\frac{1}{\theta+2 \pi j}\right)\left(\frac{1}{\theta-2 \pi k}+\frac{1}{\theta+2 \pi k}\right)\left(\frac{\theta}{2 \pi \sqrt{j k}}\right)^{-2 d} d \theta \\
& =\frac{1}{\pi} \int_{-\infty}^{\infty} \sin ^{2}\left(\frac{\theta}{2}\right)\left(\frac{1}{\theta-2 \pi j} \frac{1}{\theta+2 \pi k}+\frac{1}{\theta-2 \pi j} \frac{1}{\theta-2 \pi k}\right)\left(\frac{\theta}{2 \pi \sqrt{j k}}\right)^{-2 d} d \theta .
\end{aligned}
$$

Part b.)

$$
\begin{aligned}
& \mathbb{E}\left(\frac{B\left(\omega_{j}\right)}{\sqrt{f\left(\omega_{j}\right)}} \frac{B\left(\omega_{k}\right)}{\sqrt{f\left(\omega_{k}\right)}}\right)=\mathbb{E}\left(\frac{1}{\sqrt{f\left(\omega_{j}\right) f\left(\omega_{k}\right)}} \frac{1}{2 \pi n} \sum_{t=1}^{n} X_{t} \sin \left(\omega_{j} t\right) \sum_{s=1}^{n} X_{s} \sin \left(\omega_{k} s\right)\right) \\
& =\frac{1}{\sqrt{f\left(\omega_{j}\right) f\left(\omega_{k}\right)}} \frac{1}{2 \pi n} \int_{-\pi}^{\pi} \int_{-\pi}^{\pi} \sum_{t=1}^{n} e^{i t \theta} \sin \left(\omega_{j} t\right) \sum_{s=1}^{n} e^{i s \theta^{\prime}} \sin \left(\omega_{k} s\right) \mathbb{E}\left(d Z(\theta) \overline{d Z\left(\theta^{\prime}\right)}\right) \\
& =\frac{1}{2 \pi n} \int_{-\pi}^{\pi} \sin ^{2}\left(\frac{\theta}{2}\right) Q_{n, 1}(\theta, j) \overline{Q_{n, 1}(\theta, k)} \frac{f(\theta)}{\sqrt{f\left(\omega_{j}\right) f\left(\omega_{k}\right)}} d \theta \\
& =\frac{1}{2 \pi n^{2}} \int_{-n \pi}^{n \pi} \sin ^{2}\left(\frac{\theta}{2}\right) Q_{n, 1}(\theta, j) \overline{Q_{n, 1}(\theta, k)} \frac{f(\theta / n)}{\sqrt{f\left(\omega_{j}\right) f\left(\omega_{k}\right)}} d \theta \\
& \sim \frac{1}{2 \pi n^{2}} \int_{-n \pi}^{n \pi} \sin ^{2}\left(\frac{\theta}{2}\right) Q_{n, 1}(\theta, j) \overline{Q_{n, 1}(\theta, k)}\left(\frac{\theta}{2 \pi \sqrt{j k}}\right)^{-2 d} d \theta \\
& \longrightarrow \frac{1}{2 \pi} \int_{-\infty}^{\infty} \sin ^{2}\left(\frac{\theta}{2}\right)\left(\frac{1}{\theta-2 \pi j}-\frac{1}{\theta+2 \pi j}\right)\left(\frac{1}{\theta-2 \pi k}-\frac{1}{\theta+2 \pi k}\right)\left(\frac{\theta}{2 \pi \sqrt{j k}}\right)^{-2 d} d \theta .
\end{aligned}
$$


We note

$$
\begin{aligned}
& \int_{-\infty}^{\infty} \sin ^{2}\left(\frac{\theta}{2}\right)\left(\frac{1}{(\theta-2 \pi j)} \frac{1}{(\theta-2 \pi k)}\right)\left(\frac{\theta}{2 \pi \sqrt{j k}}\right)^{-2 d} d \theta= \\
= & \int_{-\infty}^{\infty} \sin ^{2}\left(\frac{\theta}{2}\right)\left(\frac{1}{(\theta+2 \pi j)} \frac{1}{(\theta+2 \pi k)}\right)\left(\frac{\theta}{2 \pi \sqrt{j k}}\right)^{-2 d} d \theta .
\end{aligned}
$$

Moreover,

$$
\begin{aligned}
& \int_{-\infty}^{\infty} \sin ^{2}\left(\frac{\theta}{2}\right)\left(\frac{1}{(\theta-2 \pi j)} \frac{1}{(\theta+2 \pi k)}\right)\left(\frac{\theta}{2 \pi \sqrt{j k}}\right)^{-2 d} d \theta= \\
= & \int_{-\infty}^{\infty} \sin ^{2}\left(\frac{\theta}{2}\right)\left(\frac{1}{(\theta+2 \pi j)} \frac{1}{(\theta-2 \pi k)}\right)\left(\frac{\theta}{2 \pi \sqrt{j k}}\right)^{-2 d} d \theta .
\end{aligned}
$$

Hence,

$$
\begin{aligned}
& \lim _{n \rightarrow \infty} \mathbb{E}\left(\frac{B\left(\omega_{j}\right)}{\sqrt{f\left(\omega_{j}\right)}} \frac{B\left(\omega_{k}\right)}{\sqrt{f\left(\omega_{k}\right)}}\right)= \\
= & \frac{1}{2 \pi} \int_{-\infty}^{\infty} \sin ^{2}\left(\frac{\theta}{2}\right)\left(\frac{1}{\theta-2 \pi j}-\frac{1}{\theta+2 \pi j}\right)\left(\frac{1}{\theta-2 \pi k}-\frac{1}{\theta+2 \pi k}\right)\left(\frac{\theta}{2 \pi \sqrt{j k}}\right)^{-2 d} d \theta \\
= & \frac{1}{\pi} \int_{-\infty}^{\infty} \sin ^{2}\left(\frac{\theta}{2}\right)\left(\frac{1}{(\theta-2 \pi j)} \frac{1}{(\theta-2 \pi k)}-\frac{1}{(\theta+2 \pi j)} \frac{1}{(\theta-2 \pi k)}\right)\left(\frac{\theta}{2 \pi \sqrt{j k}}\right)^{-2 d} d \theta
\end{aligned}
$$

Part c.)

$$
\begin{aligned}
& \mathbb{E}\left(\frac{A\left(\omega_{j}\right)}{\sqrt{f\left(\omega_{j}\right)}} \frac{B\left(\omega_{k}\right)}{\sqrt{f\left(\omega_{k}\right)}}\right)=\mathbb{E}\left(\frac{1}{\sqrt{f\left(\omega_{j}\right) f\left(\omega_{k}\right)}} \frac{1}{2 \pi n} \sum_{t=1}^{n} X_{t} \cos \left(\omega_{j} t\right) \sum_{s=1}^{n} X_{s} \sin \left(\omega_{k} s\right)\right) \\
& =\frac{1}{\sqrt{f\left(\omega_{j}\right) f\left(\omega_{k}\right)}} \frac{1}{2 \pi n} \int_{-\pi}^{\pi} \int_{-\pi}^{\pi} \sum_{t=1}^{n} e^{i t \theta} \cos \left(\omega_{j} t\right) \sum_{s=1}^{n} e^{i s \theta^{\prime}} \sin \left(\omega_{k} s\right) \mathbb{E}\left(d Z(\theta) \overline{d Z\left(\theta^{\prime}\right)}\right) \\
& =\frac{1}{2 \pi n} \int_{-\pi}^{\pi} \sin ^{2}\left(\frac{\theta}{2}\right) P_{n, 1}(\theta, j) \overline{Q_{n, 1}(\theta, k)} \frac{f(\theta)}{\sqrt{f\left(\omega_{j}\right) f\left(\omega_{k}\right)}} d \theta \\
& =\frac{1}{2 \pi n^{2}} \int_{-n \pi}^{n \pi} \sin ^{2}\left(\frac{\theta}{2}\right) P_{n, 1}(\theta, j) \overline{Q_{n, 1}(\theta, k)} \frac{f(\theta / n)}{\sqrt{f\left(\omega_{j}\right) f\left(\omega_{k}\right)}} d \theta \\
& \sim \frac{1}{2 \pi n^{2}} \int_{-n \pi}^{n \pi} \sin ^{2}\left(\frac{\theta}{2}\right) P_{n, 1}(\theta, j) \overline{Q_{n, 1}(\theta, k)}\left(\frac{\theta}{2 \pi \sqrt{j k}}\right)^{-2 d} d \theta \\
& \rightarrow \frac{1}{2 \pi} \int_{-\infty}^{\infty} \sin ^{2}\left(\frac{\theta}{2}\right)\left(\frac{1}{\theta-2 \pi j}+\frac{1}{\theta+2 \pi j}\right)\left(\frac{1}{\theta-2 \pi k}-\frac{1}{\theta+2 \pi k}\right)\left(\frac{\theta}{2 \pi \sqrt{j k}}\right)^{-2 d} d \theta=0 .
\end{aligned}
$$


Part d.) Similar to the above, we have by (9.6) that

$$
\begin{aligned}
\lim _{n \rightarrow \infty} \frac{1}{n^{2}} \sum_{t=1}^{n} e^{i t\left(\frac{\theta}{n}-\omega_{j}\right)} \sum_{s=1}^{n} e^{-i s\left(\frac{\theta}{n}-\omega_{k}\right)} & =\left(\frac{i(1-\cos (\theta))+\sin (\theta)}{(\theta-2 \pi j)}\right)\left(\frac{-i(1-\cos (\theta))+\sin (\theta)}{(\theta-2 \pi k)}\right) \\
& =\left(\frac{(1-\cos (\theta))^{2}+(\sin (\theta))^{2}}{(\theta-2 \pi j)(\theta-2 \pi k)}\right) \\
& =\frac{4 \sin ^{2}(\theta / 2)}{(\theta-2 \pi j)(\theta-2 \pi k)} .
\end{aligned}
$$

Therefore,

$$
\begin{aligned}
& \mathbb{E}\left(\frac{D\left(\omega_{j}\right)}{\sqrt{f\left(\omega_{j}\right)}} \frac{\overline{D\left(\omega_{j}\right)}}{\sqrt{f\left(\omega_{k}\right)}}\right)=\mathbb{E}\left(\frac{1}{\sqrt{f\left(\omega_{j}\right) f\left(\omega_{k}\right)}} \frac{1}{2 \pi n} \sum_{t=1}^{n} X_{t} e^{-i \omega_{j} t} \sum_{s=1}^{n} X_{s} e^{i \omega_{k} s}\right) \\
& =\frac{1}{\sqrt{f\left(\omega_{j}\right) f\left(\omega_{k}\right)}} \frac{1}{2 \pi n} \int_{-\pi}^{\pi} \int_{-\pi}^{\pi} \sum_{t=1}^{n} e^{i t\left(\theta-\omega_{j}\right)} \sum_{s=1}^{n} e^{-i s\left(\theta^{\prime}-\omega_{k}\right)} \mathbb{E}\left(d Z(\theta) \overline{d Z\left(\theta^{\prime}\right)}\right) \\
& =\frac{1}{\sqrt{f\left(\omega_{j}\right) f\left(\omega_{k}\right)}} \frac{1}{2 \pi n} \int_{-\pi}^{\pi} \sum_{t=1}^{n} e^{i t\left(\theta-\omega_{j}\right)} \sum_{s=1}^{n} e^{-i s\left(\theta-\omega_{k}\right)} f(\theta) d \theta \\
& =\frac{1}{2 \pi n^{2}} \int_{-n \pi}^{n \pi} \sum_{t=1}^{n} e^{i t\left(\theta / n-\omega_{j}\right)} \sum_{s=1}^{n} e^{-i s\left(\theta / n-\omega_{k}\right)} \frac{f(\theta / n)}{\sqrt{f\left(\omega_{j}\right) f\left(\omega_{k}\right)}} d \theta \\
& \longrightarrow \frac{2}{\pi} \int_{-\infty}^{\infty} \frac{\sin ^{2}(\theta / 2)}{(\theta-2 \pi j)(\theta-2 \pi k)}\left(\frac{\theta}{2 \pi \sqrt{j k}}\right)^{-2 d} d \theta, \text { By Proposition (9.9), } \\
& =L_{j, k}(d) .
\end{aligned}
$$

We note that for the next following four propositions, we have used notation that is largely attributable to Gromykov et al.(2018) and Ould Haye \& Phillippe (2019). In addition, the style of the proofs are well in the flavour of the denoted authors.

Proposition 9.12. Let $X_{t}$ be of the form of Proposition (2.3) with spectral density $f$ of the form of (2.6) where $d \in(-.5, .5)$, then $\frac{I_{n, k}^{T}\left(\omega_{j}^{\prime}\right)}{f\left(\omega_{j}^{\prime}\right)}$ and $\frac{I_{n, k}^{T}\left(\omega_{j}\right)}{f\left(\omega_{j}\right)}$ are stationary in $k$. 
Proof. Since

$$
\begin{aligned}
\mathbb{E}\left(I_{n, k}^{T}\left(\omega_{j}^{\prime}\right)\right) & =\frac{4}{3 \pi l} \sum_{s=1}^{l} \sum_{t=1}^{l} h_{t} h_{s} \gamma(t-s) e^{\omega_{j}^{\prime}(t-s)} \\
& =\frac{4}{3 \pi l}\left(\frac{3 l \gamma(0)}{8}+\sum_{p=1}^{l-1} \sum_{t=1}^{l-p} \gamma(p)\left(h_{t} h_{t+p}+h_{t} h_{t-p}\right) e^{-i \omega_{j}^{\prime} p}\right)
\end{aligned}
$$

where $t-s=p$. From the above we can see that $E\left(I_{n, k}^{T}\left(\omega_{j}^{\prime}\right)\right)$ is independent of $k$.

Now for the covariance we will note

$$
\begin{aligned}
\left|\operatorname{Cov}\left(\frac{I_{n, k}^{T}\left(\omega_{j}^{\prime}\right)}{f\left(\omega_{j}^{\prime}\right)}, \frac{I_{n, r}^{T}\left(\omega_{j}^{\prime}\right)}{f\left(\omega_{j}^{\prime}\right)}\right)\right| & \sim\left|\frac{C}{l^{2+4 d}} \sum_{t=1}^{l} \sum_{s=l}^{l} \sum_{p=1}^{l} \sum_{q=1}^{l} h_{t} h_{s} h_{p} h_{q} \operatorname{Cov}\left(X_{t} X_{s}, X_{p} X_{q}\right) e^{-i \omega_{j}^{\prime}(s-t+p-q)}\right| \\
& \leq \frac{C}{l^{2+4 d}} \sum_{t=1}^{l} \sum_{s=l}^{l} \sum_{p=1}^{l} \sum_{q=1}^{l}\left|\operatorname{Cov}\left(X_{t} X_{s}, X_{p} X_{q}\right) e^{-i \omega_{j}^{\prime}(s-t+p-q)}\right|<\infty,
\end{aligned}
$$

as Ould Haye \& Phillippe (2019) show. We note that from above $C=\frac{16}{9 \pi^{2}}$. Moreover, $\operatorname{Cov}\left(X_{t} X_{s}, X_{p} X_{q}\right)$ is a function of independent of both $k$ or $r$ solely as Brockwell and Davis (1993, Page 219, Proposition 7.3.1) show. Therefore, the above is a function of $(k-r)$ as neither the weights nor the summation operators contain any value associated with $k$ or $r$ solely.

Proposition 9.13. Let $X_{t}$ be of the form of Proposition (2.3) with spectral density $f$ of the form of (2.6) where $d \in(-.5, .5)$, then

$$
\frac{1}{m} \sum_{k=1}^{m} \frac{I_{n, k}^{T}\left(\omega_{j}^{\prime}\right)}{f\left(\omega_{j}^{\prime}\right)} \stackrel{\mathbb{P}}{\longrightarrow} L_{j, T}(d) \quad \frac{1}{m} \sum_{k=1}^{m} \frac{I_{n, k}^{T}\left(\omega_{j}\right)}{f\left(\omega_{j}\right)} \stackrel{\mathbb{P}}{\longrightarrow} L_{j, T}(d)
$$

Proof. By Proposition (4.5) we have

$$
\lim _{n \rightarrow \infty} \mathbb{E}\left(\frac{I_{n, k}^{T}\left(\omega_{j}^{\prime}\right)}{f\left(\omega_{j}^{\prime}\right)}\right)=\lim _{n \rightarrow \infty} \mathbb{E}\left|\frac{A_{T}\left(\omega_{j}^{\prime}\right)}{f\left(\omega_{j}^{\prime}\right)}\right|^{2}+\lim _{n \rightarrow \infty} \mathbb{E}\left|\frac{B_{T}\left(\omega_{j}^{\prime}\right)}{f\left(\omega_{j}^{\prime}\right)}\right|^{2}=L_{j, T}(d) .
$$


Moreover, it can be noted that $I_{n, k}^{T}\left(\omega_{j}^{\prime}\right) / f\left(\omega_{j}^{\prime}\right)$ is stationary in $k$ by Proposition (9.12) and $L_{j, T}(d)<\infty \forall d \in(-.5, .5)$. Hence, we have

$$
\lim _{n \rightarrow \infty} \mathbb{E}\left(\frac{1}{m} \sum_{k=1}^{m} \frac{I_{n, k}^{T}\left(\omega_{j}^{\prime}\right)}{f\left(\omega_{j}^{\prime}\right)}\right)=\frac{1}{m} \sum_{k=1}^{m} \lim _{n \rightarrow \infty} \mathbb{E}\left(\frac{I_{n, k}^{T}\left(\omega_{j}^{\prime}\right)}{f\left(\omega_{j}^{\prime}\right)}\right)=\frac{m}{m} L_{j, T}(d)=L_{j, T}(d)
$$

Now since $f\left(\omega_{j}^{\prime}\right) \sim c_{f}|2 \pi j / l|^{-2 d}$, and letting $C=\frac{16}{9 m \pi^{2} l^{2+4 d}}$ we have

$$
\begin{aligned}
& \operatorname{Var}\left(l^{-2 d} \frac{1}{m} \sum_{k=1}^{m} I_{n, k}^{T}\left(\omega_{j}^{\prime}\right)\right) \leq \\
& \leq C \sum_{u=1}^{m} \sum_{p=1}^{l} \sum_{q=1}^{l} \sum_{t=1}^{l} \sum_{s=1}^{l}\left|e^{-i\left((t-s) \omega_{j}^{\prime}\right)} e^{i\left((p-q) \omega_{j}^{\prime}\right)} h_{s} h_{t} h_{p} h_{q} \operatorname{Cov}\left(x_{t+k l-l} x_{s+k l-l}, x_{p+r l-l} x_{q+r l-l}\right)\right| \\
& \leq C \sum_{u=1}^{m} \sum_{p=1}^{l} \sum_{q=1}^{l} \sum_{t=1}^{l} \sum_{s=1}^{l}\left|\operatorname{Cov}\left(x_{t+k l-l} x_{s+k l-l}, x_{p+r l-l} x_{q+r l-l}\right)\right| \\
& \leq \frac{2}{m l^{4 d+1}} \sum_{p=1}^{l} \sum_{q=1}^{l} \sum_{h=1+q-l}^{l-p+1}\left|T_{l, u}(h, p, q)\right| \rightarrow 0,
\end{aligned}
$$

as $n \rightarrow \infty$ by Ould Haye \& Phillippe (2019) for $u=k-r$ and $k \geq r+2$. An important consequence of the above when $d=0$ we have

$$
\lim _{n \rightarrow \infty} \operatorname{Var}\left(\frac{1}{m} \sum_{k=1}^{m} I_{n, k}^{T}\left(\omega_{j}\right)\right)=0, \quad \lim _{n \rightarrow \infty} \operatorname{Var}\left(\frac{1}{m} \sum_{k=1}^{m} I_{n, k}^{T}\left(\omega_{j}^{\prime}\right)\right)=0 .
$$

Proposition 9.14. Assume that $l=O\left(n^{1-\delta}\right)$ with $m=O\left(n^{1-\delta^{\prime}}\right)$ for some $\delta, \delta^{\prime}>0$. Let the test statistic be defined as

$$
Q_{n, m}^{T^{\prime}}(s, d)=m^{-2 d} \sum_{j=2}^{s} \frac{I_{n}^{T}\left(\omega_{j}\right)}{\frac{1}{m} \sum_{k=1}^{m} I_{n, k}^{T}\left(\omega_{j}^{\prime}\right)} .
$$

Furthermore, assume that $X_{t}=g_{n}(t)+Y_{t}$ where $g_{n}(t)=n^{\alpha} g(t)$, where $\alpha>0, g$ is non-constant and square integratable on $[0,1]$ and $Y_{t}$ is of the form of Proposition 
(2.3) with spectral density $f$ of the form of (2.6) where $d \in(-.5, .5)$, then for any $0 \leq \varsigma \leq 1 / 2$

$$
Q_{n, m}^{T^{\prime}}(s, \varsigma) \stackrel{\mathbb{P}}{\longrightarrow} \infty
$$

Proof. The proof we provide is analogous to Ould Haye \& Phillippe (2019). We note that

$$
I_{n}^{T}\left(\omega_{j}\right)=I_{n, Y}^{T}\left(\omega_{j}\right)+n^{2 \alpha+1}\left|D_{n}^{T}(g)\right|^{2}+\operatorname{Re}\left(D_{n}^{T}(g) \overline{D_{n}^{T}(Y)}\right)
$$

where

$$
D_{n}^{T}(g)=\sum_{t=1}^{n} h_{t} g\left(\frac{t}{n}\right) e^{i 2 \pi j / n} \frac{1}{n}
$$

and

$$
\frac{1}{\sqrt{n}} D_{n}^{T}(Y)=\sum_{t=1}^{n} h_{t} Y_{t} e^{i 2 \pi j / n}
$$

Since, $Y_{t}$ is of the form of Proposition (2.3) we have

$$
I_{n, Y}^{T}\left(\omega_{j}\right) \sim f\left(\omega_{j}\right)=O\left(n^{2 d}\right) .
$$

Moreover,

$$
\left|D_{n}^{T}(g)\right|^{2} \leq \sum_{t=1}^{n} \sum_{s=1}^{n}\left|h_{t} h_{s} g\left(\frac{t}{n}\right) g\left(\frac{s}{n}\right)\right| \frac{1}{n^{2}} \leq \sum_{t=1}^{n} \sum_{s=1}^{n}\left|g\left(\frac{t}{n}\right) g\left(\frac{s}{n}\right)\right| \frac{1}{n^{2}} \sim \int_{0}^{1}|g(x)|^{2} d x<\infty .
$$

Similarly,

$$
\mathbb{E}\left|D_{n}^{T}(g) D_{n}^{T}(Y)\right| \leq\left|D_{n}^{T}(g)\right|\left(\mathbb{E}\left|D_{n}^{T}(Y)\right|\right) \leq\left|D_{n}^{T}(g)\right|\left(\mathbb{E}\left(I_{n, Y}^{T}\left(\omega_{j}\right)\right)\right)^{1 / 2} \leq C\left(\frac{n}{j}\right)^{d}
$$


Hence, the term of the greatest order of $(9.14)$ is $D_{n}^{T}(g)$. Hence with $(0 \leq \varsigma \leq .5)$

$$
n^{-2 \varsigma} I_{n}^{T}\left(\omega_{j}\right) \sim n^{2 \alpha+1-2 \varsigma} \rightarrow \infty .
$$

For the denominator, letting $I_{n, i, Y}$ denote the periodogram of the "ith" block" of the stationary process $Y_{t}$ we have

$$
\frac{1}{m} \sum_{i=1}^{m} I_{n, i, Y}^{T}\left(\omega_{j}^{\prime}\right)=O_{P}\left(l^{2 d}\right)=O_{P}(l) .
$$

Furthermore,

$$
\begin{aligned}
\frac{1}{m} \sum_{i=1}^{m} \frac{1}{l}\left|\sum_{t=1}^{n} h_{t} g_{n}(t+(i-1) l) e^{-i t \omega_{j}^{\prime}}\right|^{2} & =n^{2 \alpha} \frac{1}{m} \sum_{i=1}^{m} \frac{1}{l}\left|\sum_{t=1}^{n} h_{t} g_{n}\left(\frac{t+(i-1) l}{n}\right) e^{-i t \omega_{j}^{\prime}}\right|^{2} \\
& \leq\left. n^{2 \alpha} \frac{1}{n} \sum_{k=1}^{n}\left|\sin ^{2}\left(\frac{\pi k}{n}\right) g_{n}\left(\frac{t+(i-1) l}{n}\right)\right|^{2} e^{-i t \omega_{j}^{\prime}}\right|^{2} \\
& \leq n^{2 \alpha} \frac{1}{n} \sum_{k=1}^{n}\left|g_{n}\left(\frac{t+(i-1) l}{n}\right)\right|^{2} \sim n^{2 \alpha} \int_{0}^{1}|g(x)|^{2} d x .
\end{aligned}
$$

Moreover, for the cross-terms in (9.14) we have

$$
\begin{aligned}
& \mathbb{E}\left|\frac{1}{m} \sum_{i=1}^{m} \frac{1}{l} \sum_{t=1}^{l} h_{t} g_{n}(t+(i-1) l) e^{-i \omega_{j}^{\prime} t} \sum_{s=1}^{l} Y_{s+(i-1) l} e^{i \omega_{j}^{\prime} t}\right| \\
& \leq\left|\frac{1}{m} \sum_{i=1}^{m} \frac{1}{l} \sum_{t=1}^{l}\right| g_{n}(t+(i-1) l) e^{-i \omega_{j}^{\prime} t}|\mathbb{E}| \sum_{s=1}^{l} Y_{s+(i-1) l} e^{i t \omega_{j}^{\prime}} \mid \\
& \leq n^{\alpha} l^{d+.5} \int_{0}^{1}|g(x)| d x .
\end{aligned}
$$

Therefore, the denominator is of much smaller order than the numerator and hence we have

$$
\left(\frac{n}{l}\right)^{-2 \varsigma} \frac{I_{n}^{T}\left(\omega_{j}\right)}{\frac{1}{m} \sum_{i=1}^{m} I_{n, i}^{T}\left(\omega_{j}^{\prime}\right)} \stackrel{\mathbb{P}}{\longrightarrow} \infty .
$$

From the above we get that $Q_{n, m}^{T^{\prime}}(s, \varsigma) \stackrel{\mathbb{P}}{\longrightarrow} \infty$. 
Proposition 9.15. Assume that $l=O\left(n^{1-\delta}\right)$ with $m=O\left(n^{1-\delta^{\prime}}\right)$ for some $\delta, \delta^{\prime}>0$.

Furthermore, let the test statistic be defined as

$$
Q_{n, m}^{T^{\prime}}(s, d)=m^{-2 d} \sum_{j=2}^{s} \frac{I_{n}^{T}\left(\omega_{j}\right)}{\frac{1}{m} \sum_{k=1}^{m} I_{n, k}^{T}\left(\omega_{j}^{\prime}\right)} .
$$

Let $X_{t}=X_{t-1}+Y_{t}$, where $Y_{t}$ is of the form of (2.3) with spectral density $f$ of the form of (2.6) where $d \in(-.5, .5)$, then for any $0 \leq \varsigma \leq 1 / 2$

$$
Q_{n, m}^{T^{\prime}}(s, \varsigma) \stackrel{\mathbb{P}}{\longrightarrow} \infty
$$

Proof. We use the partial sums decomposition stated in the proof of Ould Haye \& Phillippe (2019, Theorem 2). Therefore, setting

$$
A_{t}=X_{t}, \quad b_{t}=\sin ^{2}\left(\frac{2 \pi t}{n}\right) e^{-i \omega_{j} t}
$$

we get

$$
\sum_{t=1}^{n} A_{t} b_{t}=X_{n} \sum_{t=1}^{n} \sin ^{2}\left(\frac{2 \pi t}{n}\right) e^{-i \omega_{j} t}-\sum_{t=1}^{n} Y_{t} B_{t}=-\sum_{t=1}^{n} Y_{t} B_{t} .
$$

Since

$$
\sum_{t=1}^{n} \sin ^{2}\left(\frac{2 \pi t}{n}\right) e^{-i \omega_{j} t}=0, \quad \forall j>1
$$

We note that

$$
\begin{aligned}
B_{t}=\sum_{k=1}^{t} b_{k} & =\sum_{k=1}^{t} \sin ^{2}\left(\frac{2 \pi k}{n}\right) e^{-i \omega_{j} k} \\
& =\sum_{k=1}^{t} \frac{1}{2}\left(e^{-i \omega_{j} k}-\frac{e^{-i \omega_{j+1} k}}{2}-\frac{e^{-i \omega_{j+1} k}}{2}\right) \\
& =\frac{1}{2}\left(\frac{1-e^{-i \omega_{j} t}}{e^{i \omega_{j}}-1}-\frac{1}{2} \frac{1-e^{-i \omega_{j+1} t}}{e^{i \omega_{j+1}}-1}-\frac{1}{2} \frac{1-e^{-i \omega_{j-1} t}}{e^{i \omega_{j-1}}-1}\right) \\
& \sim n^{2} \frac{1}{2}\left(\left(e^{i \omega_{j+1}}-1\right)-\frac{e^{i \omega_{j+1}}-1}{2}-\frac{e^{i \omega_{j-1}}-1}{2}\right)=n^{2} B_{t}^{*},
\end{aligned}
$$


since $\left|1-e^{-i \omega_{j}}\right|^{-2} \sim n^{2}$. Therefore,

$$
\frac{1}{n}\left|\sum_{t=1}^{n} X_{t} \sin ^{2}\left(\frac{2 \pi t}{n}\right) e^{-i \omega_{j} t}\right|^{2}=\frac{1}{n}\left|\sum_{t=1}^{n} Y_{t} B_{t}\right|^{2} \sim n\left|\sum_{t=1}^{n} Y_{t} B_{t}^{*}\right|^{2}=n^{2+2 d}\left|\sum_{t=1}^{n} \frac{Y_{t}}{n^{d+1 / 2}} B_{t}^{*}\right|^{2} .
$$

Similar to Ould Haye \& Phillippe (2019) we apply Deo (1997,Theorem 2) with

$$
g(x)=\frac{\left(1-e^{2 \pi j x}\right)}{2}-\frac{\left(1-e^{2 \pi(j+1) x}\right)}{4}-\frac{\left(1-e^{2 \pi(j-1) x}\right)}{4} .
$$

Therefore,

$$
\left|\sum_{t=1}^{n} \frac{Y_{t}}{n^{d+1 / 2}} B_{t}^{*}\right|^{2} \stackrel{\mathbb{D}}{\longrightarrow}\left|\int_{0}^{1} g(x) d \mathbb{B}(x)\right|^{2}
$$

Where $\mathbb{B}(x)$ is a fractional Brownian motion. We note that the above is a sum of positively weighted independent $X_{1}^{2}$ random variables and hence

$$
I_{n}^{T}\left(\omega_{j}\right)=O_{P}\left(n^{2+2 d}\right)
$$

For the denominator it can be seen that

$$
\frac{1}{m} \sum_{i=1}^{m} I_{n, i, Y}^{T}\left(\omega_{j}^{\prime}\right)=\frac{1}{m} \sum_{i=1}^{m} \frac{1}{l}\left|\sum_{t=1}^{l} h_{t} X_{t+(i-1) l} e^{i \omega_{j}^{\prime} t}\right|^{2}=\frac{1}{m} \sum_{i=1}^{m} \frac{1}{l}\left|\sum_{t=1}^{l} Y_{t} B_{t^{\prime}}\right|^{2} .
$$

Where

$$
B_{t^{\prime}}=\sum_{k=1}^{t} \sin ^{2}\left(\frac{2 \pi k}{l}\right) e^{-i \omega_{j}^{\prime} k}
$$

Hence,

$$
\begin{aligned}
\frac{1}{l}\left|\sum_{t=1}^{l} Y_{t} B_{t^{\prime}}\right|^{2} & \sim l\left|\sum_{t=1}^{l} Y_{t} B_{t^{\prime}}^{*}\right|^{2} \\
& \leq l\left(\left|\sum_{t=1}^{l} \frac{Y_{t}}{2}\right|^{2}+\left|\sum_{t=1}^{l} \frac{Y_{t} e^{-i \omega_{j}^{\prime} t}}{2}\right|^{2}+\left|\sum_{t=1}^{l} \frac{Y_{t} e^{\left.-i \omega_{[}(j+1)\right]^{\prime} t}}{4}\right|^{2}+\left|\sum_{t=1}^{l} \frac{Y_{t} e^{\left.-i \omega_{[}(j-1)\right]^{\prime} t}}{4}\right|^{2}\right) \\
& =l O_{P}\left(l^{2 d+1}\right)=O_{P}\left(l^{2 d+2}\right) .
\end{aligned}
$$


Therefore,

$$
\frac{1}{m} \sum_{i=1}^{m} I_{n, i, Y}^{T}\left(\omega_{j}^{\prime}\right)=O_{P}\left(l^{2 d+2}\right)
$$

Hence, we have

$$
\left(\frac{n}{l}\right)^{-2 \varsigma} \frac{I_{n}^{T}\left(\omega_{j}^{\prime}\right)}{\frac{1}{m} \sum_{i=1}^{m} I_{n, i}^{T}\left(\omega_{j}^{\prime}\right)}=\frac{O_{P}\left(n^{2 d+2-2 \varsigma}\right)}{O_{P}\left(l^{2 d+2-2 \varsigma}\right)},
$$

noting that $2 d+2-2 \varsigma>0$. Therefore, $Q_{n, m}^{T^{\prime}}(s, \varsigma) \stackrel{\mathbb{P}}{\longrightarrow} \infty$.

Lemma 9.16. Let $d \in(-.5, .5)$, then

$$
\int_{-\infty}^{\infty} \sin ^{2}\left(\frac{\theta}{2}\right) P(\theta, j) Q(\theta, k)|\theta|^{-2 d} d \theta=0
$$

Proof. We note initially $\sin ^{2}(\theta / 2)$ is an even function. Moreover,

$$
P(\theta, j)=(G(\theta+2 \pi j)+G(\theta-2 \pi j)), \quad Q(\theta, j)=(G(\theta-2 \pi j)-G(\theta+2 \pi j))
$$

are odd and even functions in $\theta$, respectively. As

$$
\begin{aligned}
G(-\theta+2 \pi j) & =\frac{1}{(-\theta+2 \pi j)(-\theta+2 \pi(j+1))(-\theta+2 \pi(j-1))} \\
& =\frac{-1}{(\theta-2 \pi j)(\theta-2 \pi(j+1))(\theta-2 \pi(j-1))} \\
& =-G(\theta-2 \pi j) .
\end{aligned}
$$

Similarly, $G(-\theta-2 \pi j)=-G(\theta+2 \pi j)$. Hence,

$$
\begin{aligned}
& P(-\theta, j)=G(-\theta+2 \pi j)+G(-\theta-2 \pi j)=-G(\theta-2 \pi j)-G(\theta+2 \pi j)=-P(\theta, j), \\
& Q(-\theta, j)=G(-\theta-2 \pi j)-G(-\theta+2 \pi j)=-G(\theta+2 \pi j)+G(\theta-2 \pi j)=Q(\theta, j) .
\end{aligned}
$$


Moreover, we note that $|\theta|^{-2 d}$ is an even function in $\theta$. Therefore, the product below is odd

$$
\sin ^{2}\left(\frac{\theta}{2}\right) P(\theta, j) Q(\theta, k)|\theta|^{-2 d}
$$

Hence,

$$
\int_{-\infty}^{\infty} \sin ^{2}\left(\frac{\theta}{2}\right) P(\theta, j) Q(\theta, k)|\theta|^{-2 d} d \theta=0
$$

Theorem 9.17. Let $X_{t}$ be of the form of Proposition (2.3) with spectral density $f$ satisfying the form of (2.6) where $d \in(-.5, .5)$. Furthermore, let

$$
\frac{A_{T}\left(\omega_{j}\right)}{\sqrt{f\left(\omega_{j}\right)}}=\sum_{t=1}^{n} z_{n t, A, j} X_{t}, \quad \frac{B_{T}\left(\omega_{j}\right)}{\sqrt{f\left(\omega_{j}\right)}}=\sum_{t=1}^{n} z_{n t, B, j} X_{t}
$$

Where

$z_{n t, A, j}=\frac{1}{\sqrt{f\left(\omega_{j}\right)}} \sqrt{\frac{4}{3 \pi n}} \sin ^{2}\left(\frac{\pi t}{n}\right) \cos \left(\omega_{j} t\right), \quad z_{n t, B, j}=\frac{1}{\sqrt{f\left(\omega_{j}\right)}} \sqrt{\frac{4}{3 \pi n}} \sin ^{2}\left(\frac{\pi t}{n}\right) \sin \left(\omega_{j} t\right)$,

then

$$
\max _{1 \leq t \leq n}\left|z_{n t, A, j}\right|=o\left(\sigma_{A, j, n}\right), \quad \quad \max _{1 \leq t \leq n}\left|z_{n t, B, j}\right|=o\left(\sigma_{B, j, n}\right) .
$$

Proof. We note that $f\left(\omega_{j}\right) \sim C_{f}\left|\frac{2 \pi j}{n}\right|^{-2 d}=C n^{2 d}=O\left(n^{2 d}\right)$ since $f$ satisfies the form of (2.6).

By Proposition (9.10) we have

$$
\sigma_{A, j}^{2}=\lim _{n \rightarrow \infty} \operatorname{Var}\left(\frac{A_{T}\left(\omega_{j}\right)}{\sqrt{f\left(\omega_{j}\right)}}\right)=O(1)
$$


Moreover, the lowest rate of convergence of $|\sin (\pi t / n)|=O(1)$ and $|\cos (2 \pi j t / n)|=$ $O(1)$ for $j$ and $t$. Hence, we have

$$
\left|z_{n t, A, j}\right|=\left|\frac{1}{\sqrt{f\left(\omega_{j}\right)}} \sqrt{\frac{4}{3 \pi n}} \sin ^{2}\left(\frac{\pi t}{n}\right) \cos \left(\omega_{j} t\right)\right|=O\left(n^{-d-.5}\right) \quad \forall t \in \mathbb{N} .
$$

Furthermore, for $d \in(-.5, .5)$

$$
\frac{\left|z_{n t, A, j}\right|}{\sigma_{A, j}}=O\left(n^{-d-.5}\right)=o(1) \quad \forall t \in \mathbb{N} .
$$

Therefore,

$$
\max _{1 \leq t \leq n}\left|z_{n t, A, j}\right|=o\left(\sigma_{A, j, n}\right)
$$

Similarly by Proposition (9.10) we have,

$$
\lim _{n \rightarrow \infty} \operatorname{Var}\left(\frac{B\left(\omega_{j}\right)}{\sqrt{f\left(\omega_{j}\right)}}\right)=O(1), \quad \forall d \in(-.5, .5)
$$

and

$$
\left|\frac{1}{\sqrt{f\left(\omega_{j}\right)}} \sqrt{\frac{4}{3 \pi n}} \sin ^{2}\left(\frac{\pi t}{n}\right) \sin \left(\omega_{j} t\right)\right|=O\left(n^{-d-.5}\right) .
$$

Therefore,

$$
\frac{\left|z_{n t, B, j}\right|}{\sigma_{B, j, n}}=O\left(n^{-d-.5}\right)=o(1), \quad \forall t \in \mathbb{N} .
$$

Hence,

$$
\max _{1 \leq t \leq n}\left|z_{n t, B, j}\right|=o\left(\sigma_{B, j, n}\right)
$$


Theorem 9.18. Let $X_{t}$ be of the form of Proposition (2.3) with spectral density $f$ of the form of (2.6) where $d \in(-.5, .5)$. Let

$$
\frac{A_{T}\left(\omega_{j}\right)}{\sqrt{f\left(\omega_{j}\right)}}=\sum_{t=1}^{n} z_{n t, A, j} X_{t}, \quad \frac{B_{T}\left(\omega_{j}\right)}{\sqrt{f\left(\omega_{j}\right)}}=\sum_{t=1}^{n} z_{n t, B, j} X_{t}
$$

Where

$z_{n t, A, j}=\frac{1}{\sqrt{f\left(\omega_{j}\right)}} \sqrt{\frac{4}{3 \pi n}} \sin ^{2}\left(\frac{\pi t}{n}\right) \cos \left(\omega_{j} t\right), \quad z_{n t, B, j}=\frac{1}{\sqrt{f\left(\omega_{j}\right)}} \sqrt{\frac{4}{3 \pi n}} \sin ^{2}\left(\frac{\pi t}{n}\right) \sin \left(\omega_{j} t\right)$,

then

$$
\sum_{t=1}^{n} z_{n t, A, j}^{2}=O\left(\sigma_{A, j, n}^{2}\right), \quad \quad \sum_{t=1}^{n} z_{n t, B, j}^{2}=O\left(\sigma_{B, j, n}^{2}\right) .
$$

Proof. We note that

$$
\sigma_{A, j, n}^{2}=O(1), \quad \sigma_{B, j, n}^{2}=O(1) .
$$

We find that the lowest order of convergence of $\sin ^{4}\left(\frac{\pi t}{n}\right) \cos ^{2}\left(\omega_{j} t\right)=O(1)$ in $t$ and $j$. Hence

$$
\sum_{t=1}^{n} \sin ^{4}\left(\frac{\pi t}{n}\right) \cos ^{2}\left(\omega_{j} t\right)=O(n)
$$

Therefore,

$$
\sum_{t=1}^{n} z_{n t, A, j}^{2}=\frac{4}{3 \pi n} \frac{1}{f\left(\omega_{j}\right)} \sum_{t=1}^{n} \sin ^{4}\left(\frac{\pi t}{n}\right) \cos ^{2}\left(\omega_{j} t\right)=O\left(n^{-2 d}\right) .
$$

Moreover for $d \in(-.5, .5)$,

$$
\frac{1}{\sigma_{A, j, n}^{2}} \sum_{t=1}^{n} z_{n t, A, j}^{2}=O\left(n^{-2 d}\right)
$$


Hence

$$
\sum_{t=1}^{n} z_{n t, A, j}^{2}=O\left(\sigma_{A, j, n}^{2}\right)
$$

Similarly, the lowest rate of convergence for $\sin ^{4}\left(\frac{\pi t}{n}\right) \sin ^{2}\left(\omega_{j} t\right)=O(1)$ for $t$ and $j$ . Therefore,

$$
\sum_{t=1}^{n} \sin ^{4}\left(\frac{\pi t}{n}\right) \sin ^{2}\left(\omega_{j} t\right)=O(n)
$$

Hence,

$$
\frac{1}{\sigma_{B, j, n}^{2}} \sum_{t=1}^{n} z_{n t, B, j}^{2}=O\left(n^{-2 d}\right)
$$

Proposition 9.19. Let $D\left(\omega_{j}\right)$ be the untapered DFT with components $A\left(\omega_{j}\right)$ and $B\left(\omega_{j}\right)$. Let $\mu_{a}=\mathbb{E}\left(a_{t}\right)$ and $C_{1}=\left(\operatorname{Var}\left(a_{t}\right) \gamma_{X}(0)\right) /\left(C_{f} 2 \pi\right)$. Moreover if $\mathbb{I}(d, j, k)=1$ when $d \neq 0, j=k$ and 0 otherwise, then

a.)

$\lim _{n \rightarrow \infty} \operatorname{Cov}\left(\frac{D^{R T}\left(\omega_{j}\right)}{\sqrt{f\left(\omega_{j}\right)}}, \frac{D^{R T}\left(\omega_{k}\right)}{\sqrt{f\left(\omega_{k}\right)}}\right)=C_{1} \mathbb{I}(d, j, k)+\left(\mu_{a}\right)^{2} \lim _{n \rightarrow \infty} \operatorname{Cov}\left(\frac{D\left(\omega_{j}\right)}{\sqrt{f\left(\omega_{j}\right)}}, \frac{D\left(\omega_{k}\right)}{\sqrt{f\left(\omega_{k}\right)}}\right)$.

b.)

$\lim _{n \rightarrow \infty} \operatorname{Cov}\left(\frac{A^{R T}\left(\omega_{j}\right)}{\sqrt{f\left(\omega_{j}\right)}}, \frac{A^{R T}\left(\omega_{k}\right)}{\sqrt{f\left(\omega_{k}\right)}}\right)=\frac{C_{1} \mathbb{I}(d, j, k)}{2}+\left(\mu_{a}\right)^{2} \lim _{n \rightarrow \infty} \operatorname{Cov}\left(\frac{A\left(\omega_{j}\right)}{\sqrt{f\left(\omega_{j}\right)}}, \frac{A\left(\omega_{k}\right)}{\sqrt{f\left(\omega_{k}\right)}}\right)$.

c.)

$\lim _{n \rightarrow \infty} \operatorname{Cov}\left(\frac{B^{R T}\left(\omega_{j}\right)}{\sqrt{f\left(\omega_{j}\right)}}, \frac{B^{R T}\left(\omega_{k}\right)}{\sqrt{f\left(\omega_{k}\right)}}\right)=\frac{C_{1} \mathbb{I}(d, j, k)}{2}+\left(\mu_{a}\right)^{2} \lim _{n \rightarrow \infty} \operatorname{Cov}\left(\frac{B\left(\omega_{j}\right)}{\sqrt{f\left(\omega_{j}\right)}}, \frac{B\left(\omega_{k}\right)}{\sqrt{f\left(\omega_{k}\right)}}\right)$. 
d.)

$$
\lim _{n \rightarrow \infty} \operatorname{Cov}\left(\frac{A^{R T}\left(\omega_{j}\right)}{\sqrt{f\left(\omega_{j}\right)}}, \frac{B^{R T}\left(\omega_{k}\right)}{\sqrt{f\left(\omega_{k}\right)}}\right)=0 .
$$

Proof. Part a.) We find

$$
\begin{aligned}
& \operatorname{Cov}\left(\frac{D^{R T}\left(\omega_{j}\right)}{\sqrt{f\left(\omega_{j}\right)}}, \frac{D^{R T}\left(\omega_{k}\right)}{\sqrt{f\left(\omega_{k}\right)}}\right)=\mathbb{E}\left(\frac{D^{R T}\left(\omega_{j}\right)}{\sqrt{f\left(\omega_{j}\right)}} \frac{\overline{D^{R T}\left(\omega_{k}\right)}}{\sqrt{f\left(\omega_{k}\right)}}\right) \\
& \quad=\frac{1}{2 \pi n \sqrt{f\left(\omega_{j}\right) f\left(\omega_{k}\right)}} \int_{-\pi}^{\pi} \int_{-\pi}^{\pi} \sum_{t=1}^{n} e^{-i t\left(\theta-\omega_{j}\right)} \sum_{s=1}^{n} e^{i s\left(\theta^{\prime}-\omega_{k}\right)} \mathbb{E}\left(d Z(\theta) \overline{\left.d Z\left(\theta^{\prime}\right)\right)}\right) \\
& =\frac{1}{2 \pi n \sqrt{f\left(\omega_{j}\right) f\left(\omega_{k}\right)}} \int_{-\pi}^{\pi} \sum_{t=1}^{n} e^{-i t\left(\theta-\omega_{j}\right)} \sum_{s=1}^{n} e^{i s\left(\theta-\omega_{k}\right)} f_{R T}(\theta) d \theta \\
& =\frac{1}{2 \pi n \sqrt{f\left(\omega_{j}\right) f\left(\omega_{k}\right)}} \int_{-\pi}^{\pi} S_{n}(\theta, j, k) f_{R T}(\theta) d \theta
\end{aligned}
$$

where

$$
S_{n}(\theta, j, k)=\sum_{t=1}^{n} e^{i t\left(\theta-\omega_{j}\right)} \sum_{s=1}^{n} e^{-i s\left(\theta-\omega_{k}\right)}
$$

Furthermore, using (9.6) and Proposition (9.8) we have

$$
\lim _{n \rightarrow \infty} \frac{1}{n^{2}} S_{n}\left(\frac{\theta}{n}, j, k\right)=\frac{4 \sin ^{2}(\theta / 2)}{(\theta-2 \pi j)(\theta-2 \pi k)} .
$$

Moreover, if $d \in(0, .5)$ as $n \rightarrow \infty$

$$
\frac{f_{R T}(\theta / n)}{\sqrt{f\left(\omega_{j}\right) f\left(\omega_{k}\right)}} \sim\left(\mathbb{E}\left(a_{1}\right)\right)^{2}\left(\frac{\theta}{2 \pi \sqrt{j k}}\right)^{-2 d} .
$$

Therefore, if $d \in(0, .5)$ solving for the limiting value we make a change of variable with the above by setting $\lambda=\frac{\theta}{n} \rightarrow d \lambda=\frac{d \theta}{n}$. Applying this in conjunction with the 
results from Proposition (9.9) we have

$$
\begin{aligned}
& \lim _{n \rightarrow \infty} \operatorname{Cov}\left(\frac{D^{R T}\left(\omega_{j}\right)}{\sqrt{f\left(\omega_{j}\right)}}, \frac{D^{R T}\left(\omega_{k}\right)}{\sqrt{f\left(\omega_{k}\right)}}\right)= \\
& =\lim _{n \rightarrow \infty} \frac{1}{2 \pi n^{2} \sqrt{f\left(\omega_{j}\right) f\left(\omega_{k}\right)}} \int_{-n \pi}^{n \pi} S_{n}\left(\frac{\theta}{n}, j, k\right) f_{R T}\left(\frac{\theta}{n}\right) d \theta \\
& =\left(\mathbb{E}\left(a_{1}\right)\right)^{2} \frac{2}{\pi} \int_{-n \pi}^{n \pi} \frac{\sin ^{2}(\theta / 2)}{(\theta-2 \pi j)(\theta-2 \pi k)}\left(\frac{\theta}{2 \pi \sqrt{j k}}\right)^{-2 d} d \theta \\
& =\left(\mathbb{E}\left(a_{1}\right)\right)^{2} \operatorname{Cov}\left(\frac{D\left(\omega_{j}\right)}{\sqrt{f\left(\omega_{j}\right)}}, \frac{D\left(\omega_{k}\right)}{\sqrt{f\left(\omega_{k}\right)}}\right) .
\end{aligned}
$$

Therefore,

$$
\lim _{n \rightarrow \infty} \operatorname{Cov}\left(\frac{D^{R T}\left(\omega_{j}\right)}{\sqrt{f\left(\omega_{j}\right)}}, \frac{D^{R T}\left(\omega_{k}\right)}{\sqrt{f\left(\omega_{k}\right)}}\right)=\left(\mu_{a}\right)^{2} \lim _{n \rightarrow \infty} \operatorname{Cov}\left(\frac{D\left(\omega_{j}\right)}{\sqrt{f\left(\omega_{j}\right)}}, \frac{D\left(\omega_{k}\right)}{\sqrt{f\left(\omega_{k}\right)}}\right) .
$$

Moreover, in the case of $d=0$ and $j \neq k$ we have

$$
\lim _{n \rightarrow \infty} \operatorname{Cov}\left(\frac{D^{R T}\left(\omega_{j}\right)}{\sqrt{f\left(\omega_{j}\right)}}, \frac{D^{R T}\left(\omega_{k}\right)}{\sqrt{f\left(\omega_{k}\right)}}\right)=C L_{j, k}(0)=0
$$

By Hurvich \& Beltrao (1993). Furthermore, when $d=0$ and $j=k$

$$
\lim _{n \rightarrow \infty} \operatorname{Cov}\left(\frac{D^{R T}\left(\omega_{j}\right)}{\sqrt{f\left(\omega_{j}\right)}}, \frac{D^{R T}\left(\omega_{k}\right)}{\sqrt{f\left(\omega_{k}\right)}}\right)=C_{1}+\mu_{a}^{2} L_{j}(0)=C_{1}+\mu_{a}^{2}
$$

We note that the proofs for the limiting forms of the covariances of the $A^{R T}$ and $B^{R T}$ components are analogous to the above.

Proposition 9.20. Let $X_{t}$ be of the form of Proposition (2.3) with spectral density $f$ satisfying (2.6) where $d \in[0, .5)$. Moreover, let the taper $a_{t}$ be of the form of $(4.5)$ where the weights are fixed. Furthermore, assume that

$$
\frac{A^{R T}\left(\omega_{j}\right)}{\sqrt{f\left(\omega_{j}\right)}}=\frac{1}{\sqrt{f\left(\omega_{j}\right)}} \frac{1}{\sqrt{2 \pi n}} \sum_{t=1}^{n} a_{t} X_{t} \cos \left(\omega_{j} t\right)
$$




$$
\frac{B^{R T}\left(\omega_{j}\right)}{\sqrt{f\left(\omega_{j}\right)}}=\frac{1}{\sqrt{f\left(\omega_{j}\right)}} \frac{1}{\sqrt{2 \pi n}} \sum_{t=1}^{n} a_{t} X_{t} \sin \left(\omega_{j} t\right)
$$

and

$$
\lim _{n \rightarrow \infty} \sigma_{R T A, j, n}^{2}(d)=\sigma_{R T A, j}^{2}(d) \quad \lim _{n \rightarrow \infty} \sigma_{R T B, j, n}^{2}(d)=\sigma_{R T B, j}^{2}(d),
$$

where

$$
\sigma_{R T A, j, n}^{2}(d)=\operatorname{Var}\left(\frac{A^{R T}\left(\omega_{j}\right)}{\sqrt{f\left(\omega_{j}\right)}}\right), \quad \sigma_{R T B, j, n}^{2}(d)=\operatorname{Var}\left(\frac{B^{R T}\left(\omega_{j}\right)}{\sqrt{f\left(\omega_{j}\right)}}\right)
$$

Moreover, we note that for simplicity $\sigma_{R T A, j, n}^{2}(d)=\sigma_{R T A, j, n}^{2}, \sigma_{R T B, j, n}^{2}(d)=\sigma_{R T B, j, n}^{2}$. Let

$\mathcal{D}_{R T, n}=\left(\frac{A^{R T}\left(\omega_{1}\right)}{\sigma_{R T A, 1, n} \sqrt{f\left(\omega_{1}\right)}}, . ., \frac{A^{R T}\left(\omega_{s}\right)}{\sigma_{R T A, s, n} \sqrt{f\left(\omega_{s}\right)}}, \frac{B^{R T}\left(\omega_{1}\right)}{\sigma_{R T B, 1, n} \sqrt{f\left(\omega_{1}\right)}}, . ., \frac{B^{R T}\left(\omega_{s}\right)}{\sigma_{R T B, s, n} \sqrt{f\left(\omega_{s}\right)}}\right)$. Assume $a=\left(a_{1}, \ldots, a_{n}\right)$ is the vector of $n$ tapers and let $P\left(\mathcal{D}_{R T, n} \leq x \mid a\right)$ be the multivariate probability distribution function of the random vector $\mathcal{D}_{R T, n}$ conditioned upon the random taper $a$, then

$$
P\left(\mathcal{D}_{R T, n} \leq x \mid a\right) \stackrel{\mathbb{P}_{a}}{\longrightarrow} \Phi_{2 s}(x)
$$

Where $\Phi_{2 s}(x)$ is the multivariate Gaussian random vector cumulative distribution function of order $2_{s}$ with its covariance matrix given in Proposition (9.21).

Proof.

$$
\frac{A_{R T}\left(\omega_{j}\right)}{\sqrt{f\left(\omega_{j}\right)}}=\sum_{t=1}^{n} z_{n t, R T A, j} X_{t} \quad \frac{B_{R T}\left(\omega_{j}\right)}{\sqrt{f\left(\omega_{j}\right)}}=\sum_{t=1}^{n} z_{n t, R T B, j} X_{t} .
$$


Where the weights are

$$
z_{n t, R T A, j}=\frac{1}{\sqrt{2 \pi n f\left(\omega_{j}\right)}} \cos \left(\omega_{j} t\right) a_{t}, \quad z_{n t, R T B, j}=\frac{1}{\sqrt{2 \pi n f\left(\omega_{j}\right)}} \sin \left(\omega_{j} t\right) a_{t} .
$$

Hence, it is trivial to see

$$
\max _{1 \leq t \leq n}\left|\cos \left(\omega_{j} t\right)\right|=O(1), \quad \max _{1 \leq t \leq n}\left|\sin \left(\omega_{j} t\right)\right|=O(1) .
$$

Furthermore, since $f$ satisfies (2.6) we have

$$
f\left(\omega_{j}\right) \sim C_{f}\left|\omega_{j}\right|^{-2 d}=O\left(n^{2 d}\right)
$$

we have

$$
\max _{1 \leq t \leq n}\left|z_{n t, R T A, j}\right|=O_{P_{a}}\left(n^{-.5-d}\right), \quad \max _{1 \leq t \leq n}\left|z_{n t, R T B, j}\right|=O_{P_{a}}\left(n^{-.5-d}\right) .
$$

Hence,

$$
\begin{aligned}
\sigma_{R T A, j, n}^{2} & =\operatorname{Var}\left(\frac{A^{R T}\left(\omega_{j}\right)}{\sqrt{f\left(\omega_{j}\right)}}\right)=\mathbb{E}\left(\frac{A^{R T}\left(\omega_{j}\right)}{\sqrt{f\left(\omega_{j}\right)}}\right)^{2} \\
& =\frac{1}{2 \pi n} \frac{1}{f\left(\omega_{j}\right)} \sum_{t=1}^{n} \sum_{s=1}^{n} a_{t} a_{s} \gamma_{X}(t-s) \cos \left(\omega_{j}(t)\right) \cos \left(\omega_{j}(s)\right) .
\end{aligned}
$$

Which behaves analogously with

$$
\begin{aligned}
& \frac{1}{2 \pi n} \frac{1}{f\left(\omega_{j}\right)} \sum_{t=1}^{n} \sum_{s=1}^{n} a_{t} a_{s} \gamma_{X}(t-s) \cos \left(\omega_{j}(t-s)\right)= \\
& \frac{1}{2 \pi n} \frac{1}{f\left(\omega_{j}\right)}\left(\gamma_{X}(0) \sum_{t=1}^{n} a_{t}^{2}+2 \sum_{h=1}^{n-1} \gamma_{X}(h) \cos \left(\omega_{j} h\right) \sum_{t=1}^{n-h} a_{t} a_{t+h}\right) .
\end{aligned}
$$

We first start by evaluating the $2 \sum_{h=1}^{n-1} \gamma_{X}(h) \cos \left(\omega_{j} h\right) \sum_{t=1}^{n-h} a_{t} a_{t+h}$ term. Focusing on the mean we can see that

$$
\mathbb{E}\left(\frac{2 \sum_{h=1}^{n-1} \gamma_{X}(h) \cos \left(\omega_{j} h\right) \sum_{t=1}^{n-h} a_{t} a_{t+h}}{2 \pi n f\left(\omega_{j}\right)}\right)=\frac{\left(\mathbb{E}\left(a_{1}\right)\right)^{2}}{\pi n f\left(\omega_{j}\right)} \sum_{h=1}^{n-1}(n-h) \gamma_{X}(h) \cos \left(\omega_{j} h\right) .
$$


Noting that as $\gamma_{X}(h)=\int_{-\pi}^{\pi} f(\theta) e^{i h \theta} d \theta$, and $\cos \left(\omega_{j} h\right)=(1 / 2)\left(e^{-i h \omega_{j}}+e^{i h \omega_{j}}\right)$ we have

$$
\begin{aligned}
& \frac{\left(\mathbb{E}\left(a_{1}\right)\right)^{2}}{\pi n f\left(\omega_{j}\right)} \sum_{h=1}^{n-1}(n-h) \gamma_{X}(h) \cos \left(\omega_{j} h\right)= \\
& =\frac{\left(\mathbb{E}\left(a_{1}\right)\right)^{2}}{\pi f\left(\omega_{j}\right)} \int_{-\pi}^{\pi} \sum_{h=1}^{n-1}\left(1-\frac{h}{n}\right)\left(e^{i h\left(\theta-\omega_{j}\right)}+e^{i h\left(\theta+\omega_{j}\right)}\right) f(\theta) d \theta .
\end{aligned}
$$

Moreover, we have

$$
\sum_{h=1}^{n-1}\left(1-\frac{h}{n}\right) e^{-i \theta h}=\frac{1}{2}\left(\sum_{h=1-n}^{n-1}\left(1-\frac{|h|}{n}\right) e^{-i \theta h}-1\right)=\frac{1}{2 n} \frac{\sin ^{2}((\theta n) / 2)}{\sin ^{2}(\theta / 2)}-\frac{1}{2} .
$$

Hence, we can see

$$
\frac{1}{n} \sum_{h=1}^{n-1}\left(1-\frac{h}{n}\right)\left(e^{i h\left(\theta / n-\omega_{j}\right)}+e^{i h\left(\theta / n+\omega_{j}\right)}\right)=\frac{1}{2 n^{2}} \frac{\sin ^{2}(\theta / 2)}{\sin ^{2}\left(\frac{\theta}{2 n}+\frac{\omega_{j}}{2}\right)}+\frac{1}{2 n^{2}} \frac{\sin ^{2}(\theta / 2)}{\sin ^{2}\left(\frac{\theta}{2 n}-\frac{\omega_{j}}{2}\right)}-\frac{1}{n} .
$$

Moreover, by (9.6) we have the above converging to

$$
\left(\frac{2 \sin (\theta / 2)}{(\theta+2 \pi j)}\right)^{2}+\left(\frac{2 \sin (\theta / 2)}{(\theta-2 \pi j)}\right)^{2}
$$

Hence,

$$
\lim _{n \rightarrow \infty} \frac{1}{n} \sum_{h=1}^{n-1}\left(1-\frac{h}{n}\right) e^{i h\left(\frac{\theta}{n}-\omega_{j}\right)} \frac{f(\theta / n)}{f\left(\omega_{j}\right)}=2 \sin ^{2}\left(\frac{\theta}{2}\right)\left(\frac{1}{(\theta+2 \pi j)^{2}}+\frac{1}{(\theta-2 \pi j)^{2}}\right)\left(\frac{\theta}{2 \pi j}\right)^{-2 d} .
$$

Therefore, by performing a change of variable and implementing Proposition (9.9) we have

$$
\begin{aligned}
& \lim _{n \rightarrow \infty} \frac{\left(\mathbb{E}\left(a_{1}\right)\right)^{2}}{\pi f\left(\omega_{j}\right)} \int_{-\pi}^{\pi} \sum_{h=1}^{n-1}\left(1-\frac{h}{n}\right)\left(e^{i h\left(\theta-\omega_{j}\right)}+e^{i h\left(\theta+\omega_{j}\right)}\right) f(\theta) d \theta= \\
& =\frac{\left(2 \mathbb{E}\left(a_{1}\right)\right)^{2}}{\pi} \int_{-\infty}^{\infty} \sin ^{2}\left(\frac{\theta}{2}\right)^{2}\left(\frac{1}{(\theta+2 \pi j)^{2}}+\frac{1}{(\theta-2 \pi j)^{2}}\right)\left(\frac{\theta}{2 \pi j}\right)^{-2 d} d \theta .
\end{aligned}
$$


We now focus on the variance of the term under analysis. We note that

$$
\operatorname{Cov}\left(a_{t} a_{t+h}, a_{s} a_{s+p}\right) \neq 0
$$

only when

- $t=s$ and $h=p$,

- $t=p$ and $h=s$,

- $t=s$ and $h \neq p$,

- $t \neq s, h=p$ and $s=t+h$,

as $t=s+p \& s=t+h$ cannot occur simultaneously. Furthermore, when $t=s$ and $p=h$ we have

$$
\operatorname{Cov}\left(a_{t} a_{t+h}, a_{t} a_{t+h}\right)=\mathbb{E}\left(a_{t}^{2}\right) \mathbb{E}\left(a_{t+h}^{2}\right)-\left(\mathbb{E}\left(a_{t}\right)\right)^{2}\left(\mathbb{E}\left(a_{t+h}\right)\right)^{2}=\left(\operatorname{Var}\left(a_{1}\right)\right)^{2}
$$

Moreover, when $t=s$ and $p \neq h$

$\operatorname{Cov}\left(a_{t} a_{t+h}, a_{t} a_{t+p}\right)=\mathbb{E}\left(a_{t}^{2}\right)\left(\mathbb{E}\left(a_{t+h}\right)\right)^{2}-\left(\mathbb{E}\left(a_{t}\right)\right)^{2}\left(\mathbb{E}\left(a_{t+h}\right)\right) \mathbb{E}\left(a_{t+h}\right)=\operatorname{Var}\left(a_{1}\right)\left(\mathbb{E}\left(a_{1}\right)\right)^{2}$

When $t \neq s$ and $h \neq p$

$$
\operatorname{Cov}\left(a_{t} a_{t+h}, a_{t} a_{t+p}\right)=\mathbb{E}\left(a_{t}\right) \mathbb{E}\left(a_{t+h}\right) \mathbb{E}\left(a_{s}\right) \mathbb{E}\left(a_{s+p}\right)-\mathbb{E}\left(a_{t}\right) \mathbb{E}\left(a_{t+h}\right) \mathbb{E}\left(a_{s}\right) \mathbb{E}\left(a_{s+p}\right)=0
$$


Hence,

$$
\operatorname{Cov}\left(a_{t} a_{t+h}, a_{s} a_{s+p}\right)= \begin{cases}\left(\operatorname{Var}\left(a_{1}\right)\right)^{2} & t=s, p=h \\ \operatorname{Var}\left(a_{1}\right)\left(\mathbb{E}\left(a_{1}\right)\right)^{2} & t=s, h \neq p \\ \operatorname{Var}\left(a_{1}\right)\left(\mathbb{E}\left(a_{1}\right)\right)^{2} & t \neq s, h=p \\ \operatorname{Var}\left(a_{1}\right)\left(\mathbb{E}\left(a_{1}\right)\right)^{2} & t=p, s=h \\ 0 & t \neq s, h \neq p\end{cases}
$$

For $t=s$ and $h=p$ we have

$$
\begin{aligned}
& \operatorname{Cov}\left(\sum_{h=1}^{n-1} \gamma_{X}(h) \cos \left(\omega_{j} h\right) \sum_{t=1}^{n-h} a_{t} a_{t+h}, \sum_{p=1}^{n-1} \gamma_{X}(p) \cos \left(\omega_{j} p\right) \sum_{s=1}^{n-p} a_{s} a_{s+p}\right) \\
& =\sum_{h=1}^{n-1} \sum_{p=1}^{n-1} \gamma_{X}(h) \gamma_{X}(p) \cos \left(\omega_{j} h\right) \cos \left(\omega_{j} p\right) \sum_{t=1}^{n-h} \sum_{s=1}^{n-p} \operatorname{Cov}\left(a_{t} a_{t+h}, a_{s} a_{s+p}\right) \\
& =\left(\operatorname{Var}\left(a_{1}\right)\right)^{2} \sum_{h=1}^{n-1} \sum_{t=1}^{n-h} \gamma_{X}^{2}(h) \cos ^{2}\left(\omega_{j} h\right) .
\end{aligned}
$$

Utilizing the fact that $\gamma(h) \sim C_{\gamma} h^{2 d-1}$, we have

$$
\begin{aligned}
& \frac{\left(\mathbb{E}\left(a_{1}^{2}\right)\right)^{2}}{n^{2+4 d}}\left|\sum_{h=1}^{n}(n-h) \gamma_{X}^{2}(h) \cos ^{2}\left(\omega_{j} h\right)\right| \\
& \leq \frac{\left(\mathbb{E}\left(a_{1}^{2}\right)\right)^{2}}{n^{1+4 d}} \sum_{h=1}^{n} \gamma_{X}^{2}(h) \\
& \sim \frac{C}{n^{2+4 d}} n^{4 d} \\
& =o(1) .
\end{aligned}
$$

Hence, we can see that when $t=s$ and $h=p$ we have

$$
\operatorname{Var}\left(\frac{1}{\pi n f\left(\omega_{j}\right)}\left(\sum_{h=1}^{n-1} \gamma_{X}(h) \cos \left(\omega_{j} h\right) \sum_{t=1}^{n-h} a_{t} a_{t+h}\right)\right)=O\left(n^{-2}\right)=o(1)
$$


In the case of $t=s$ and $h \neq p$, letting $\operatorname{Var}\left(a_{1}\right)\left(\mathbb{E}\left(a_{1}\right)\right)^{2}=\mu$

$$
\begin{aligned}
& \operatorname{Cov}\left(\sum_{h=1}^{n-1} \gamma_{X}(h) \cos \left(\omega_{j} h\right) \sum_{t=1}^{n-h} a_{t} a_{t+h}, \sum_{p=1}^{n-1} \gamma_{X}(p) \cos \left(\omega_{j} p\right) \sum_{s=1}^{n-p} a_{s} a_{s+p}\right) \\
& =\sum_{h=1}^{n-1} \sum_{p=1}^{n-1} \gamma_{X}(h) \gamma_{X}(p) \cos \left(\omega_{j} h\right) \cos \left(\omega_{j} p\right) \sum_{t=1}^{n-h} \sum_{s=1}^{n-p} \operatorname{Cov}\left(a_{t} a_{t+h}, a_{t} a_{t+p}\right) \\
& =\mu \sum_{h=1}^{n-1} \sum_{p=1}^{n-1} \sum_{t=1}^{n-h} \gamma_{X}(h) \cos \left(\omega_{j} h\right) \gamma_{X}(p) \cos \left(\omega_{j} p\right) .
\end{aligned}
$$

Furthermore,

$$
\begin{aligned}
& \frac{\mu}{n^{2+4 d}} \sum_{h=1}^{n-1} \sum_{p=1}^{n-1} \sum_{t=1}^{n-h} \gamma_{X}(h) \cos \left(\omega_{j} h\right) \gamma_{X}(p) \cos \left(\omega_{j} p\right) \\
& \leq \frac{\mu}{n^{1+4 d}} \sum_{h=1}^{n} \sum_{p=1}^{n}\left|\gamma_{X}(h) \gamma_{X}(p)\right| \\
& \sim \frac{C}{n^{1+4 d}} n^{4 d} \\
& =o(1) .
\end{aligned}
$$

Hence, when $t=s$ and $h \neq p$

$$
\operatorname{Var}\left(\frac{1}{\pi n f\left(\omega_{j}\right)}\left(\sum_{h=1}^{n-1} \gamma_{X}(h) \cos \left(\omega_{j} h\right) \sum_{t=1}^{n-h} a_{t} a_{t+h}\right)\right)=O\left(n^{-1}\right)=o(1)
$$

Moreover, it is trivial to see $t \neq s$ and $h=p$, or $t=s$ and $h=s$, we have

$$
\operatorname{Var}\left(\frac{1}{\pi n f\left(\omega_{j}\right)}\left(\sum_{h=1}^{n-1} \gamma_{X}(h) \cos \left(\omega_{j} h\right) \sum_{t=1}^{n-h} a_{t} a_{t+h}\right)\right)=o(1)
$$

With the asymptotic behaviour of the mean and the variance we can see that

$$
\frac{1}{\pi n f\left(\omega_{j}\right)} \sum_{h=1}^{n-1} \gamma_{X}(h) \cos \left(\omega_{j} h\right) \sum_{t=1}^{n-h} a_{t} a_{t+h}=O_{P_{a}}(1)
$$


Now evaluating

$$
\mathbb{E}\left(\frac{1}{2 \pi n} \frac{1}{f\left(\omega_{j}\right)}\left(\gamma_{X}(0) \sum_{t=1}^{n} a_{t}^{2}\right)\right)=\frac{\mathbb{E}\left(a_{1}^{2}\right)}{2 \pi f\left(\omega_{j}\right)} \gamma_{X}(0)=O\left(n^{-2 d}\right)
$$

and

$$
\operatorname{Var}\left(\frac{\gamma_{X}(0)}{2 \pi n} \frac{\sum_{t=1}^{n} a_{t}^{2}}{f\left(\omega_{j}\right)}\right)=\frac{\gamma_{X}(0)}{4 \pi^{2} n^{2}\left(f\left(\omega_{j}\right)\right)^{2}} \sum_{t=1}^{n} \operatorname{Var}\left(a_{t}^{2}\right)=O\left(n^{-4 d-1}\right),
$$

as $a_{t}^{2}$ and $a_{s}^{2}$ are independent for $t \neq s$. Hence,

$$
\frac{1}{2 \pi n} \frac{1}{f\left(\omega_{j}\right)}\left(\gamma_{X}(0) \sum_{t=1}^{n} a_{t}^{2}\right)=O_{P_{a}}\left(n^{-2 d}\right)
$$

Therefore,

$$
\sigma_{R T A, j, n}^{2}=O_{P_{a}}\left(n^{-2 d}\right)+O_{P_{a}}(1)
$$

Hence, for $d \in[0, .5), \sigma_{R T A, j, n}^{2}=O_{P_{a}}(1)$.

Therefore,

$$
\frac{\max _{1 \leq t \leq n}\left|z_{n t, R T A, j}\right|}{\sigma_{R T A, j, n}}=O_{P_{a}}\left(n^{-.5-d}\right)=o_{P_{a}}(1), \text { if } d \in[0, .5) .
$$

A similar proof can be shown for the sine component of the randomly tapered DFT.

To prove the second part of condition (i) we note

$$
\mathbb{E}\left(\sum_{t=1}^{n} z_{n t, R T A, j}^{2}\right)=\frac{1}{f\left(\omega_{j}\right)} \frac{\mathbb{E}\left(a_{t}^{2}\right)}{2 \pi n} \sum_{t=1}^{n} \cos ^{2}\left(\omega_{j} t\right)=\frac{1}{f\left(\omega_{j}\right)} \frac{\mathbb{E}\left(a_{t}^{2}\right)}{4 \pi}=O\left(n^{-2 d}\right),
$$

and

$$
\operatorname{Var}\left(\sum_{t=1}^{n} z_{n t, R T A, j}^{2}\right)=\frac{\sum_{t=1}^{n} \cos ^{4}\left(\omega_{j} t\right)}{\left(f\left(\omega_{j}\right)\right)^{2}} \frac{\operatorname{Var}\left(a_{t}^{2}\right)}{(2 \pi n)^{2}}=\frac{1}{\left(f\left(\omega_{j}\right)\right)^{2}} \frac{\operatorname{Var}\left(a_{t}^{2}\right)}{(2 \pi n)^{2}}\left(\frac{3 n}{8}\right)=O\left(n^{-4 d-1}\right) .
$$


Hence,

$$
\sum_{t=1}^{n} z_{n t, R T A, j}^{2}=O_{P_{a}}\left(n^{-2 d}\right) .
$$

Therefore,

$$
\frac{\sum_{t=1}^{n} z_{n t, R T A, j}^{2}}{\sigma_{R T A, j, n}^{2}}=O_{P_{a}}\left(n^{-2 d}\right) .
$$

Similarly, we can show

$$
\frac{\sum_{t=1}^{n} z_{n t, R T B, j}^{2}}{\sigma_{R T B, j, n}^{2}}=O_{P_{a}}\left(n^{-2 d}\right) .
$$

From the above we can see that condition (i) of Theorem (2.5) is satisfied with probability $P_{a}$ and therefore by Theorem (2.5) we have

$$
\begin{array}{r}
\left(\frac{A^{R T}\left(\omega_{1}\right)}{\sigma_{R T A, 1, n} \sqrt{f\left(\omega_{1}\right)}}, . ., \frac{A^{R T}\left(\omega_{s}\right)}{\sigma_{R T A, s, n} \sqrt{f\left(\omega_{s}\right)}}, \frac{B^{R T}\left(\omega_{1}\right)}{\sigma_{R T B, 1, n} \sqrt{f\left(\omega_{1}\right)}}, . ., \frac{B^{R T}\left(\omega_{s}\right)}{\sigma_{R T B, s, n} \sqrt{f\left(\omega_{s}\right)}}\right) \stackrel{\mathbb{D}}{\longrightarrow} \\
\stackrel{\mathbb{D}}{\longrightarrow} N_{2 s}\left(0, \Sigma_{2 s}\right) .
\end{array}
$$

We note that the above can also be articulated equivalently as

$$
P\left(\mathcal{D}_{R T, n} \leq x \mid a\right) \stackrel{\mathbb{P}_{a}}{\longrightarrow} \Phi_{2 s}(x)
$$

Where $\Phi_{2 s}(x)$ is the CDF of a multivariate normal distribution with the covariance matrix $\Sigma_{2 s}$ is composed with components taken from the second order moments displayed within Proposition (9.19). We have withheld providing this covariance matrix here as we elaborate more on this within Proposition (9.21). 
Proposition 9.21. Let $X_{t}$ be of the form of Proposition (2.3) with spectral density $f$ satisfying (2.6) where $d \in[0, .5)$. Assume the taper $a_{t}$ is of the form of (4.5) and is assumed to be random, then

$$
\left(\frac{A^{R T}\left(\omega_{1}\right)}{\sigma_{R T A, 1, n} \sqrt{f\left(\omega_{1}\right)}}, . ., \frac{A^{R T}\left(\omega_{s}\right)}{\sigma_{R T A, s, n} \sqrt{f\left(\omega_{s}\right)}}, \frac{B^{R T}\left(\omega_{1}\right)}{\sigma_{R T B, 1, n} \sqrt{f\left(\omega_{1}\right)}}, . ., \frac{B^{R T}\left(\omega_{s}\right)}{\sigma_{R T B, s, n} \sqrt{f\left(\omega_{s}\right)}}\right) \stackrel{\mathbb{P}}{\longrightarrow} \mathcal{V}_{R T}
$$

Where the covariance matrix is

$$
\mathcal{V}_{R T}=\left(Z_{1}^{R T}(1), \ldots, Z_{1}^{R T}(s), Z_{2}^{R T}(1), \ldots, Z_{2}^{R T}(s)\right)
$$

follows a $N_{2 s}\left(0, \Sigma_{2 s, 2 s}^{R T}\right)$ distribution. Where

$$
\Sigma_{2 s, 2 s}^{R T}=\left[\begin{array}{cc}
A^{R T} & 0_{s} \\
0_{s} & B^{R T}
\end{array}\right] .
$$

With

$$
\mathcal{A}^{R T}=\left[\begin{array}{ccccc}
1 & a_{12} & a_{13} & \ldots & a_{1 s} \\
a_{21} & 1 & a_{23} & \ldots & a_{2 s} \\
\ldots & \ldots & \ldots & \ldots & \ldots \\
a_{s 1} & a_{s 2} & a_{s 3} & \ldots & 1
\end{array}\right], \mathcal{B}^{R T}=\left[\begin{array}{ccccc}
1 & b_{12} & b_{13} & \ldots & a_{1 s} \\
b_{21} & 1 & b_{23} & \ldots & a_{2 s} \\
\ldots & \ldots & \ldots & \ldots & \ldots \\
b_{n 1} & b_{s 2} & b_{s 3} & \ldots & 1
\end{array}\right],
$$

where $\mathcal{A}^{R T}$ and $\mathcal{B}^{R T}$ are the covariance matrices for the vectors

$$
\left(\frac{A^{R T}\left(\omega_{1}\right)}{\sigma_{R T A, 1, n} \sqrt{f\left(\omega_{1}\right)}}, \ldots, \frac{A^{R T}\left(\omega_{s}\right)}{\sigma_{R T A, s, n} \sqrt{f\left(\omega_{s}\right)}}\right) \text { and }\left(\frac{B^{R T}\left(\omega_{1}\right)}{\sigma_{R T B, 1, n} \sqrt{f\left(\omega_{1}\right)}}, \ldots, \frac{B^{R T}\left(\omega_{s}\right)}{\sigma_{R T B, s, n} \sqrt{f\left(\omega_{s}\right)}}\right) \text {, }
$$

respectively. The matrix $0_{s}$ is the cross covariance of the vectors. The entries of the covariance matrices are

$$
a_{j, k}=\left(\mu_{a}\right)^{2} \lim _{n \rightarrow \infty} \operatorname{Cov}\left(\frac{A\left(\omega_{j}\right)}{\sigma_{R T A, j, n} \sqrt{f\left(\omega_{j}\right)}}, \frac{A\left(\omega_{k}\right)}{\sigma_{R T A, k, n} \sqrt{f\left(\omega_{k}\right)}}\right),
$$




$$
b_{j, k}=\left(\mu_{a}\right)^{2} \lim _{n \rightarrow \infty} \operatorname{Cov}\left(\frac{B\left(\omega_{j}\right)}{\sigma_{R T B, j, n} \sqrt{f\left(\omega_{j}\right)}}, \frac{B\left(\omega_{k}\right)}{\sigma_{R T B, k, n} \sqrt{f\left(\omega_{k}\right)}}\right) .
$$

Proof. For simplicity let

$$
\mathcal{D}_{n}=\left(\frac{A^{R T}\left(\omega_{1}\right)}{\sigma_{R T A, 1, n} \sqrt{f\left(\omega_{1}\right)}}, . ., \frac{A^{R T}\left(\omega_{s}\right)}{\sigma_{R T A, s, n} \sqrt{f\left(\omega_{s}\right)}}, \frac{B^{R T}\left(\omega_{1}\right)}{\sigma_{R T B, 1, n} \sqrt{f\left(\omega_{1}\right)}}, . ., \frac{B^{R T}\left(\omega_{s}\right)}{\sigma_{R T B, s, n} \sqrt{f\left(\omega_{s}\right)}}\right)
$$

be a random vector of $2 s$ components. By Proposition (9.20) we have the culmulative distribution function of $\mathcal{D}_{n}$ converging in probability $P_{a}$ to the culmulative distribution of the normal random vector given in Proposition (9.20) above:

$$
P\left(\mathcal{D}_{n} \leq x \mid a\right) \stackrel{\mathbb{P}}{\longrightarrow} \Phi_{2 s}(x)
$$

From the above

$$
\lim _{n \rightarrow \infty} \mathbb{E}\left(P\left(\mathcal{D}_{n} \leq x \mid a\right)\right)=\mathbb{E}\left(\Phi_{2 s}(x)\right)=\Phi_{2 s}(x) .
$$

Hence,

$$
\lim _{n \rightarrow \infty} P\left(\mathcal{D}_{n} \leq x\right)=\Phi_{2 s}(x)
$$

Hence, we can see that $\mathcal{D}_{n}$ converges in distribution. For the components of the covariance matrix we let

$$
\sigma_{R T A, j, k}^{2}=\lim _{n \rightarrow \infty} \sigma_{R T A, j, k, n}^{2}=\lim _{n \rightarrow \infty} \operatorname{Cov}\left(\frac{A^{R T}\left(\omega_{j}\right)}{\sqrt{f\left(\omega_{j}\right)}}, \frac{A^{R T}\left(\omega_{k}\right)}{\sqrt{f\left(\omega_{k}\right)}}\right)
$$

and

$$
\sigma_{R T B, j, k}^{2}=\lim _{n \rightarrow \infty} \sigma_{R T B, j, k, n}^{2}=\lim _{n \rightarrow \infty} \operatorname{Cov}\left(\frac{B^{R T}\left(\omega_{j}\right)}{\sqrt{f\left(\omega_{j}\right)}}, \frac{B^{R T}\left(\omega_{k}\right)}{\sqrt{f\left(\omega_{k}\right)}}\right)
$$


From Part b.) and Part c.) of Proposition (9.19) we have

$$
a_{j, k}=\frac{\sigma_{R T A, j, k}^{2}}{\sigma_{R T A, j} \sigma_{R T A, k}},
$$

and

$$
b_{j, k}=\frac{\sigma_{R T B, j, k}^{2}}{\sigma_{R T B, j} \sigma_{R T B, k}} .
$$

It is important to note that in the case of short memory $d=0$ the random vector

$$
\mathcal{V}_{R T}=\left(Z_{1}^{R T}(1), \ldots ., Z_{1}^{R T}(s), Z_{2}^{R T}(1), \ldots ., Z_{2}^{R T}(s)\right)
$$

follows a multivariate standard normal distribution.

Proposition 9.22. Let $X_{t}$ be of the form of Proposition (2.3) with spectral density $f$ of the form of (2.6) where $d \in[0, .5)$, then

$$
\left(\frac{I_{n}^{R T}\left(\omega_{1}\right)}{f\left(\omega_{1}\right)}, . ., \frac{I_{n}^{R T}\left(\omega_{s}\right)}{f\left(\omega_{s}\right)}\right) \stackrel{\mathbb{D}}{\longrightarrow}\left(E_{1}, \ldots, E_{s}\right) .
$$

Where

$$
E_{j}=\left(\sigma_{R T A, j}^{2}\left(Z_{1}^{R T}(j)\right)^{2}+\sigma_{R T B, j}^{2}\left(Z_{2}^{R T}(j)\right)^{2}\right)
$$

Proof. We note that

$$
I_{n}^{R T}\left(\omega_{j}\right)=\left|A^{R T}\left(\omega_{j}\right)\right|+\left|B^{R T}\left(\omega_{j}\right)\right| .
$$

Utilizing the Continuous Mapping Theorem in conjunction with Proposition (9.21) we have that

$$
\left(\frac{I_{n}^{R T}\left(\omega_{1}\right)}{f\left(\omega_{1}\right)}, . ., \frac{I_{n}^{R T}\left(\omega_{s}\right)}{f\left(\omega_{s}\right)}\right) \stackrel{\mathbb{D}}{\longrightarrow}\left(E_{1}, \ldots, E_{s}\right) .
$$


Where

$$
\begin{aligned}
E_{j} & =\left|\sigma_{R T A, j} Z_{1}^{R T}(j)-i \sigma_{R T B, j} Z_{2}^{R T}(j)\right|^{2} \\
& =\left(\sigma_{R T A, j} Z_{1}^{R T}(j)\right)^{2}+\left(\sigma_{R T B, j} Z_{2}^{R T}(j)\right)^{2} .
\end{aligned}
$$

Proposition 9.23. Let $X_{t}$ be of the form of Proposition (2.3) with spectral density $f$ of the form of (2.6) where $d \in[0, .5)$, then

a.)

$$
\frac{1}{m} \sum_{k=1}^{m} \frac{I_{n, i}^{R T}\left(\omega_{j}^{\prime}\right)}{f\left(\omega_{j}^{\prime}\right)} \stackrel{\mathbb{P}}{\longrightarrow} \sigma_{R T, j}^{2} .
$$

b.)

$$
m^{-2 d} \sum_{j=1}^{s}\left(\frac{I_{n}^{R T}\left(\omega_{1}\right)}{\frac{1}{m} \sum_{k=1}^{m} I_{n, k}^{R T}\left(\omega_{j}^{\prime}\right)}\right) \stackrel{\mathbb{D}}{\longrightarrow} Q^{R T}(s, d) .
$$

Where

$$
Q^{R T}(s, d)=\sum_{j=1}^{s} \frac{E_{j}}{\sigma_{R T, j}^{2}}
$$

Proof. For part a.) we find that similar to the proof of Proposition (9.21) Let 


$$
\begin{aligned}
\gamma_{R T}(h)=\operatorname{Cov}\left(a_{t} X_{t}, a_{t+h} X_{t+h}\right) & \text { then } \\
\mathbb{E}\left(I_{n, k}^{R T}\left(\omega_{j}^{\prime}\right)\right) & =\frac{1}{2 \pi l} \sum_{t=1}^{l} \sum_{s=1}^{l} \gamma_{R T}(t-s) e^{-i \omega_{j}^{\prime}(t-s)} \\
& =\frac{1}{2 \pi l} \sum_{h=1}^{l-1} \sum_{t=1}^{l-|h|} \gamma_{R T}(h) e^{-i \omega_{j}^{\prime} h} \\
& =\frac{1}{2 \pi l} \sum_{h=1-l}^{l-1}(l-|h|) \int_{-\pi}^{\pi} f_{R T}(\theta) e^{-i\left(\omega_{j}-\theta\right) h} d \theta \\
& =\frac{1}{2 \pi l} \int_{-\pi}^{\pi} J_{l}\left(\theta-\omega_{j}^{\prime}\right) f_{R T}(\theta) d \theta
\end{aligned}
$$

where

$$
J_{l}\left(\theta-\omega_{j}^{\prime}\right)=\sum_{h=1-l}^{l-1}(l-|h|) e^{-i\left(\theta-\omega_{j}^{\prime}\right) h}=\frac{\sin ^{2}\left(\left(\theta-\omega_{j}^{\prime}\right) l / 2\right)}{\sin ^{2}\left(\theta-\omega_{j}^{\prime}\right) / 2},
$$

by Beran et al.(2013, Page 327). Moreover,

$$
\frac{1}{2 \pi l} \int_{-\pi}^{\pi} J_{l}\left(\theta-\omega_{j}^{\prime}\right) f_{R T}(\theta) d \theta=\frac{1}{2 \pi l} \int_{-l \pi}^{l \pi} J_{l}\left(\lambda / l-\omega_{j}^{\prime}\right) f_{R T}(\lambda / l) d \lambda
$$

as $\theta=\frac{\lambda}{l} \rightarrow d \theta=\frac{d \lambda}{l}$. Furthermore,

$$
\mathbb{E}\left(\frac{I_{n, k}^{R T}\left(\omega_{j}^{\prime}\right)}{f\left(\omega_{j}^{\prime}\right)}\right)=\frac{1}{2 \pi l} \int_{-l \pi}^{l \pi} J_{n}\left(\lambda / l-\omega_{j}^{\prime}\right) \frac{f_{R T}(\lambda / l)}{f\left(\omega_{j}^{\prime}\right)} d \lambda .
$$

Now

$$
\frac{f_{R T}(\lambda / l)}{f\left(\omega_{j}^{\prime}\right)} \sim \frac{\operatorname{Var}\left(a_{1}\right) \gamma_{X}(0)}{2 \pi C_{f}(2 \pi j)^{-2 d} l^{2 d}}+\left(\mathbb{E}\left(a_{1}\right)\right)^{2}\left(\frac{\lambda}{2 \pi j}\right)^{-2 d}
$$

In the case of $d=0$ we have

$$
\lim _{n \rightarrow \infty} \mathbb{E}\left(\frac{I_{n, k}^{R T}\left(\omega_{j}^{\prime}\right)}{f\left(\omega_{j}^{\prime}\right)}\right)=\frac{\operatorname{Var}\left(a_{1}\right) \gamma_{X}(0)}{2 \pi C_{f}}+\left(\mathbb{E}\left(a_{1}\right)\right)^{2}
$$


When $d \in(0, .5)$

$$
\frac{f_{R T}(\lambda / l)}{f\left(\omega_{j}^{\prime}\right)} \sim\left(\mathbb{E}\left(a_{1}\right)\right)^{2}\left(\frac{\lambda}{2 \pi j}\right)^{-2 d}
$$

Furthermore, we note that

$$
\lim _{n \rightarrow \infty} \frac{1}{2 \pi l^{2}} J_{l}\left(\theta / l-\omega_{j}^{\prime}\right)=\frac{\sin ^{2}(\theta / 2)}{(\theta-2 \pi j)^{2}}
$$

Hence, Proposition (9.9) we have

$$
\lim _{n \rightarrow \infty} \mathbb{E}\left(\frac{I_{n, k}^{R T}\left(\omega_{j}^{\prime}\right)}{f\left(\omega_{j}^{\prime}\right)}\right)=\left(\mathbb{E}\left(a_{1}\right)\right)^{2} \frac{2}{\pi} \int_{-\infty}^{\infty} \frac{\sin ^{2}(\lambda / 2)}{(\lambda-2 \pi j)^{2}}\left(\frac{\lambda}{2 \pi j}\right)^{-2 d} d \lambda=\left(\mathbb{E}\left(a_{1}\right)\right)^{2} L_{j}(d) .
$$

Therefore, for $d \in[0, .5)$

$$
\lim _{n \rightarrow \infty} \mathbb{E}\left(\frac{I_{n, k}^{R T}\left(\omega_{j}^{\prime}\right)}{f\left(\omega_{j}^{\prime}\right)}\right)=\sigma_{R T, j}^{2}
$$

Furthermore, by the stationarity of $\frac{I_{n, k}^{R T}\left(\omega_{j}^{\prime}\right)}{f\left(\omega_{j}^{\prime}\right)}$ in $k$ we can see that as $n \rightarrow \infty$

$$
\mathbb{E}\left(\frac{1}{m} \sum_{k=1}^{m} \frac{I_{n, k}^{R T}\left(\omega_{j}^{\prime}\right)}{f\left(\omega_{j}^{\prime}\right)}\right) \rightarrow \sigma_{R T, j}^{2}
$$

Moreover, since $\left|a_{t}\right| \leq C, C>0$

$$
\begin{aligned}
& \left|\operatorname{Cov}\left(\frac{I_{n, i}^{R T}\left(\omega_{j}^{\prime}\right)}{f\left(\omega_{j}^{\prime}\right)}, \frac{I_{n, k}^{R T}\left(\omega_{j}^{\prime}\right)}{f\left(\omega_{j}^{\prime}\right)}\right)\right| \leq \\
& \leq \frac{1}{f\left(\omega_{j}^{\prime}\right) f\left(\omega_{j}^{\prime}\right)}\left(\frac{1}{(2 \pi l)^{2}} \sum_{t} \sum_{s} \sum_{p} \sum_{q}\left|\mathbb{E}\left(a_{t} a_{s} a_{p} a_{q} X_{t} X_{s} X_{p} X_{q}\right) e^{-i \omega_{j}^{\prime}(t-s)} e^{-i \omega_{j}^{\prime}(p-q)}\right|\right) \\
& \leq\left(\frac{1}{f\left(\omega_{j}^{\prime}\right) f\left(\omega_{j}^{\prime}\right)} \frac{1}{(2 \pi l)^{2}} \sum_{t=1}^{l} \sum_{s=1}^{l} \sum_{p=1}^{l} \sum_{q=1}^{l}\left|\mathbb{E}\left(X_{t+(i-1) l} X_{s+(i-1) l} X_{p+(k-1) l} X_{q+(k-1) l}\right)\right|\right) .
\end{aligned}
$$

Which is the same upper bound used in Ould Haye \& Phillippe (2019). Therefore,

$$
\frac{1}{m^{2}} \operatorname{Cov}\left(\sum_{i=1}^{m} \frac{I_{n, i}^{R T}\left(\omega_{j}^{\prime}\right)}{f\left(\omega_{j}^{\prime}\right)}, \sum_{k=1}^{m} \frac{I_{n, k}^{R T}\left(\omega_{j}^{\prime}\right)}{f\left(\omega_{j}^{\prime}\right)}\right) \longrightarrow 0
$$


Hence,

$$
\frac{1}{m} \sum_{k=1}^{m} \frac{I_{n, k}^{R T}\left(\omega_{j}^{\prime}\right)}{f\left(\omega_{j}^{\prime}\right)} \stackrel{\mathbb{P}}{\longrightarrow} \sigma_{R T, j}^{2}
$$

Part b.)

By applying the Continuous Mapping Theorem, Slutsky's Theorem, and Proposition (9.22) we have

$$
\left(\frac{I_{n}^{R T}\left(\omega_{1}\right) / f\left(\omega_{1}\right)}{\frac{1}{m} \sum_{k=1}^{m} I_{n, k}^{R T}\left(\omega_{1}^{\prime}\right) / f\left(\omega_{1}^{\prime}\right)}, \ldots, \frac{I_{n}^{R T}\left(\omega_{s}\right) / f\left(\omega_{j}\right)}{\frac{1}{m} \sum_{k=1}^{m} I_{n, k}^{R T}\left(\omega_{s}^{\prime}\right) / f\left(\omega_{s}^{\prime}\right)}\right) \stackrel{\mathbb{P}}{\longrightarrow}\left(\frac{E_{1}}{\sigma_{R T, 1}^{2}}, . ., \frac{E_{s}}{\sigma_{R T, s}^{2}}\right) .
$$

By the Continuous Mapping Theorem we have

$$
\sum_{j=1}^{s} \frac{I_{n}^{R T}\left(\omega_{j}\right) / f\left(\omega_{j}\right)}{\frac{1}{m} \sum_{k=1}^{m} I_{n, k}^{R T}\left(\omega_{j}^{\prime}\right) / f\left(\omega_{j}^{\prime}\right)} \stackrel{\mathbb{D}}{\longrightarrow} \sum_{j=1}^{s} \frac{E_{j}}{\sigma_{R T, j}^{2}}=Q^{R T}(s, d) .
$$

Furthermore, since

$$
\left(\frac{f\left(\omega_{j}\right)}{f\left(\omega_{j}^{\prime}\right)} m^{-2 d}\right) \sim 1
$$

we have

$$
\left(\frac{f\left(\omega_{j}\right)}{f\left(\omega_{j}^{\prime}\right)} m^{-2 d}\right) \sum_{j=1}^{s} \frac{I_{n}^{R T}\left(\omega_{j}\right) / f\left(\omega_{j}\right)}{\frac{1}{m} \sum_{k=1}^{m} I_{n, k}^{R T}\left(\omega_{j}^{\prime}\right) / f\left(\omega_{j}^{\prime}\right)} \stackrel{\mathbb{P}}{\longrightarrow} Q^{R T}(s, d)
$$




\section{Appendix B}

\section{$10.1 \quad$ Numerical Code}

Within this section we have included the numerical code that we have utilized to generate the vast majority of the plots included within this research in respect to the statistics built with the cosine bell taper. We note that the "Qnmcb" function provides a specified amount ("nsim") of Monte Carlo simulations for the p-values generated from the $Q_{n, m}^{T}(s), Q_{n, m}^{T}(s, d)$, and $Q_{n, m}^{T^{\prime}}(s)$ tests under the null and alternative hypotheses of these tests. We have included plot functions at the bottom of the code that allow for one to generate plots that we have included within our research. The manner in which we have embedded the code allows for the reader to seamlessly extract the code and implement it into R by simply copying and pasting the entire code. Therefore, by running the entire script we have attached, one is able to generate these plots. However, in the case where one would like to tailor the parameters utilized within the statistics, the "Qnmcb" function will have to be loaded. Upon loading this function one is free to alter the parameters and produce the desired results. 
\# nsim = amount of simulations.

\# $\mathrm{s}=$ amount of periodogram ordinates $\mathrm{s}$.

$\# \mathrm{~m}=$ amount of blocks.

$\# \mathrm{n}=$ sample size.

\# $a=$ ar coefficient under null for first and alter of second or memory parameter d for alter of first and null of second.

\# proc $=$ takes on values of "first" or "second". Refers to which procedure is being simulated.

\# hyp = takes on values of "null" for null hypothesis for first and second procedure

\# or "alt" for alternative hypothesis for first procedure, "alt1" for alter of second procedure with deterministic trend

\# or "alt2" for alternative of second procedure for random walk. Refers to which hypothesis is being simulated.

\# fo = takes on values of "yes" or " $n$ ". Refers to if the first periodogram ordinate is removed or kept.

install.packages ("fracdiff")

library (fracdiff)

Qnmcb=function(nsim, s,m,n, a, proc, hyp, fo) \{

simmingfunction=function (nsim, s, m, n, a, proc, hyp) \{

FirstOrdinateTaperedPeriodogram=function $(n, x, s)\{$

$1=\operatorname{length}(\mathrm{x})$

$i=\operatorname{complex}(r e a l=0$, imaginary $=1)$

FirstOrdinateTaperedPeriodogram J $=$ function $(j)\{$

$\operatorname{return}((4 /(3 * \operatorname{pi} * 1)) *(\operatorname{Mod}(\operatorname{sum}(x *(.5 *(1-\cos (2 * \operatorname{pi} * \operatorname{seq}(1,1) / 1))) * \exp (-i *(2 * \operatorname{pi} * j / 1 *(\operatorname{seq}(1,1))))))) \wedge(2))$

\}

return (sapply (seq $(2, \mathrm{~s})$, FirstOrdinateTaperedPeriodogramJ, simplify=TRUE))

\}

BlockSortingFunction=function $(\mathrm{n}, 1, \mathrm{~m}, \mathrm{x})\{$

BlockValues=function $(j)\{$

blockmat $=x[(1+(j-1) * 1):(j * 1)]$

return (blockmat)

\}

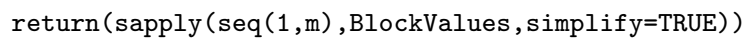

\}

FirstOrdinateDenominatorTaperedPeriodogram $=$ function $(\mathrm{s}, \mathrm{m}, \mathrm{x})\{$

$\mathrm{n}=$ length $(\mathrm{x})$ \#

$l=f \operatorname{loor}(n / m)$

xmatrix=BlockSortingFunction $(\mathrm{n}, 1, \mathrm{~m}, \mathrm{x})$

FirstOrdinateTaperedBlockPeriodogram=function(h) \{

$\operatorname{return}(($ FirstOrdinateTaperedPeriodogram $(\mathrm{n}, \operatorname{xmatrix}[, \mathrm{h}], \mathrm{s})))$

\}

return (sapply (seq $(1, m)$, FirstOrdinateTaperedBlockPeriodogram)) 


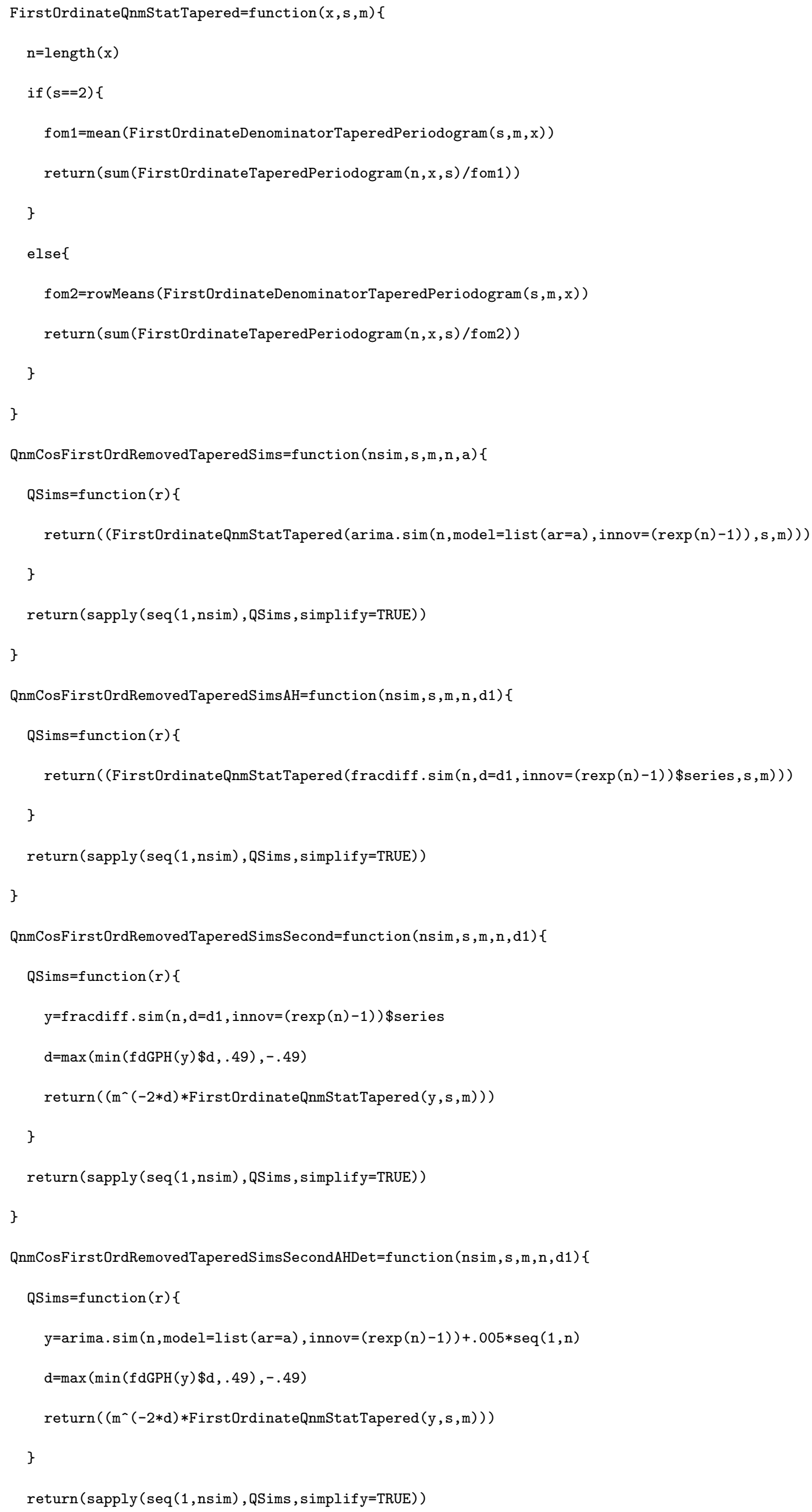


QSims=function $(r)\{$

$\mathrm{y}=$ cumsum $(\operatorname{arima} \cdot \operatorname{sim}(\mathrm{n}, \operatorname{model}=1$ ist $(\operatorname{ar}=\mathrm{a})$, innov $=(\operatorname{rexp}(\mathrm{n})-1)))$

$\mathrm{d}=\max (\min (\mathrm{fdGPH}(\mathrm{y}) \$ \mathrm{~d}, .49),-.49)$

return $\left(\left(\mathrm{m}^{\wedge}(-2 * \mathrm{~d}) *\right.\right.$ FirstOrdinateQnmStatTapered $\left.\left.(\mathrm{y}, \mathrm{s}, \mathrm{m})\right)\right)$

\}

return ( $\operatorname{sapply}($ seq $(1, \mathrm{nsim})$, QSims, simplify=TRUE))

\}

if ( (proc=="first")\&(hyp=="null") ) \{

return(QnmCosFirstOrdRemovedTaperedSims (nsim, s, m, n, a))

\}

if ( (proc=="first") \&(hyp=="alt" ) )\{

return(QnmCosFirstOrdRemovedTaperedSimsAH(nsim, s,m,n, a))

\}

if $($ (proc=="second" $) \&($ hyp=="null" $))\{$

return (QnmCosFirstOrdRemovedTaperedSimsSecond (nsim, s,m,n, a))

\}

if $($ (proc=="second" $) \&($ hyp=="alt1" $))\{$

return(QnmCosFirstOrdRemovedTaperedSimsSecondAHDet (nsim, s, m, n, a))

\}

if ( (proc==" second") \& (hyp=="alt2" ) )\{

return(QnmCosFirstOrdRemovedTaperedSimsSecondAHRandWalk (nsim, s, m, n, a))

\}

\}

Qnmcbf of inal=function(nsim,s,m,n, d,proc, hyp, fo) \{

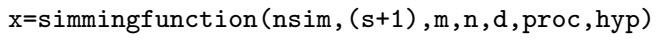

if $(($ proc=="first" $) \&($ hyp=="null" $))\{$

limdist=limitdist $(0, s, f o)$

pvaling=1-limdist $(x)$

return (ecdf (pvaling))

\}

else if ((proc=="first")\&(hyp=="alt")) \{

limdist $=\operatorname{limitdist~}(0, \mathrm{~s}, \mathrm{fo})$

pvaling=1-limdist $(x)$

return (ecdf (pvaling))

\}

else if ((proc==" second")\&(hyp=="null" ) \{ 


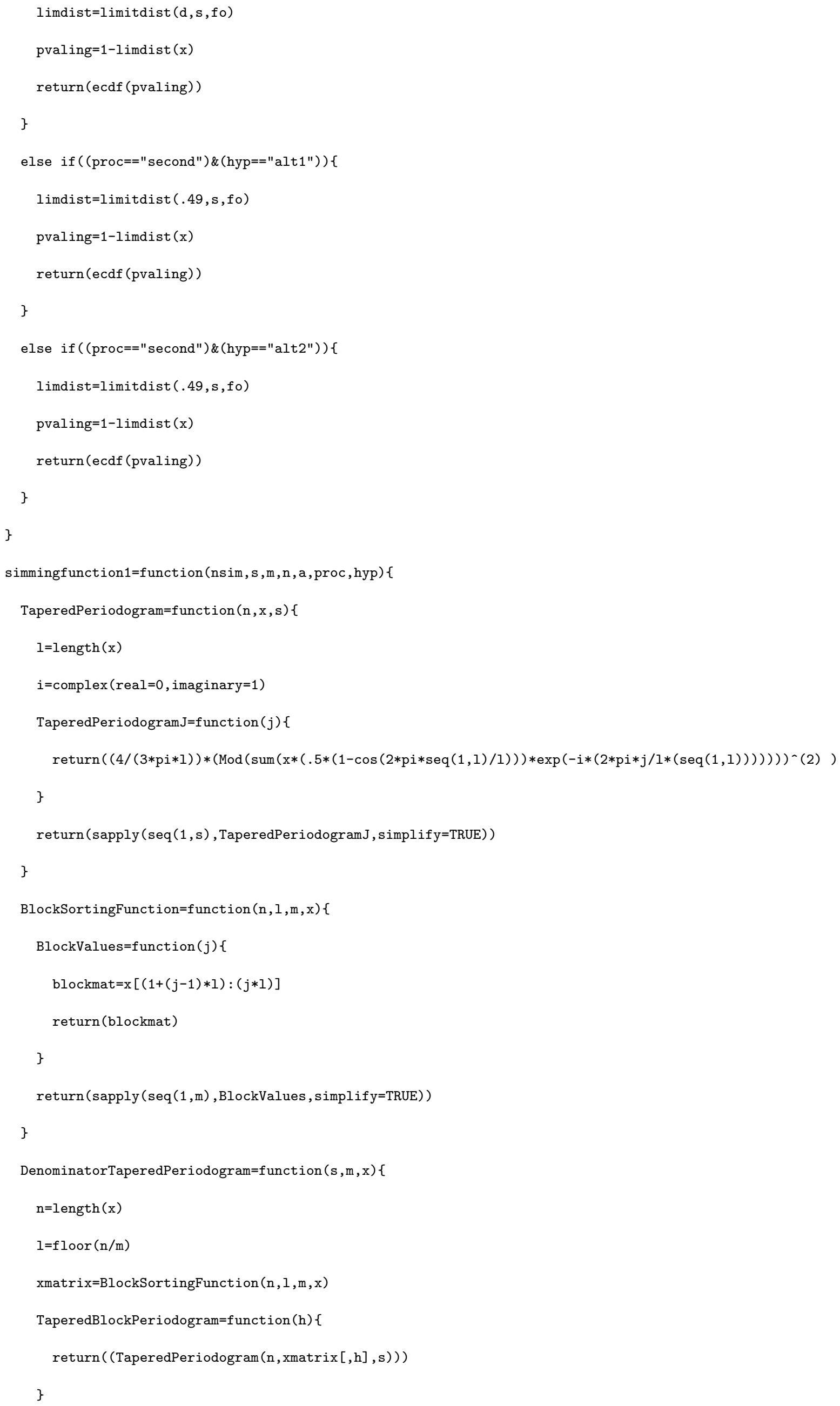




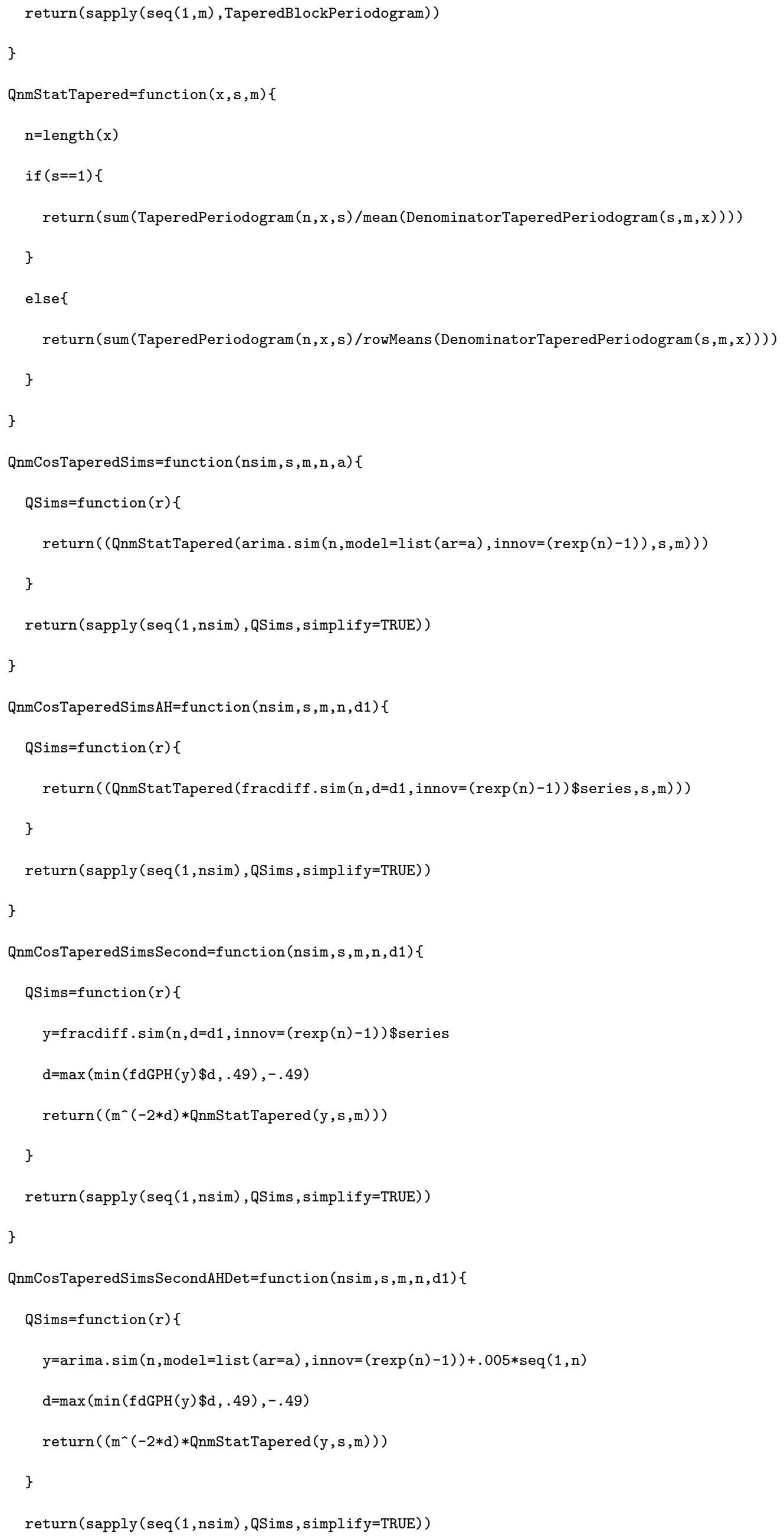


QSims=function $(r)\{$

$\mathrm{y}=\operatorname{cumsum}(\operatorname{arima} \cdot \operatorname{sim}(\mathrm{n}, \operatorname{model}=\mathrm{list}(\operatorname{ar}=\mathrm{a})$, innov $=(\operatorname{rexp}(\mathrm{n})-1)))$

$\mathrm{d}=\max (\min (\mathrm{fdGPH}(\mathrm{y}) \$ \mathrm{~d}, .49),-.49)$

$\operatorname{return}\left(\left(\mathrm{m}^{\wedge}(-2 * \mathrm{~d}) *\right.\right.$ QnmStatTapered $\left.\left.(\mathrm{y}, \mathrm{s}, \mathrm{m})\right)\right)$

\}

return ( $\operatorname{sapply}(\operatorname{seq}(1, \mathrm{nsim})$, QSims , simplify=TRUE) $)$

\}

if ( (proc=="first") \& (hyp=="null") ) \{

return(QnmCosTaperedSims (nsim, s, m, n, a))

\}

if $($ (proc=="first" $) \&($ hyp=="alt" $))\{$

return(QnmCosTaperedSimsAH(nsim, s, m, n, a))

\}

if $($ (proc=="second") \& (hyp=="null" ) $)\{$

return (QnmCosTaperedSimsSecond (nsim, s, m, n, a))

\}

if $(($ proc $=="$ second" $) \&($ hyp=="alt1" $))\{$

return(QnmCosTaperedSimsSecondAHDet (nsim, s,m,n, a))

\}

if $(($ proc $=="$ second" $) \&($ hyp=="alt2" $))\{$

return(QnmCosTaperedSimsSecondAHRandWalk (nsim, s, m, n, a))

\}

\}

Qnmcbfinal=function(nsim,s,m,n,d,proc,hyp,fo) \{

$\mathrm{x}=$ simmingfunction 1 (nsim, $\mathrm{s}, \mathrm{m}, \mathrm{n}, \mathrm{d}, \mathrm{proc}, \mathrm{hyp})$

if $(($ proc $=="$ first" $) \&($ hyp $=="$ null" $))\{$

limdist $=\operatorname{limitdist}(0, \mathrm{~s}, \mathrm{fo})$

pvaling=1-limdist $(\mathrm{x})$

return (ecdf (pvaling))

\}

else if ((proc=="first")\&(hyp=="alt"))\{

limdist $=\operatorname{limitdist~}(0, \mathrm{~s}, \mathrm{fo})$

pvaling=1-limdist $(x)$

return (ecdf (pvaling))

else if ((proc==" second")\&(hyp=="null" $)\{$ 


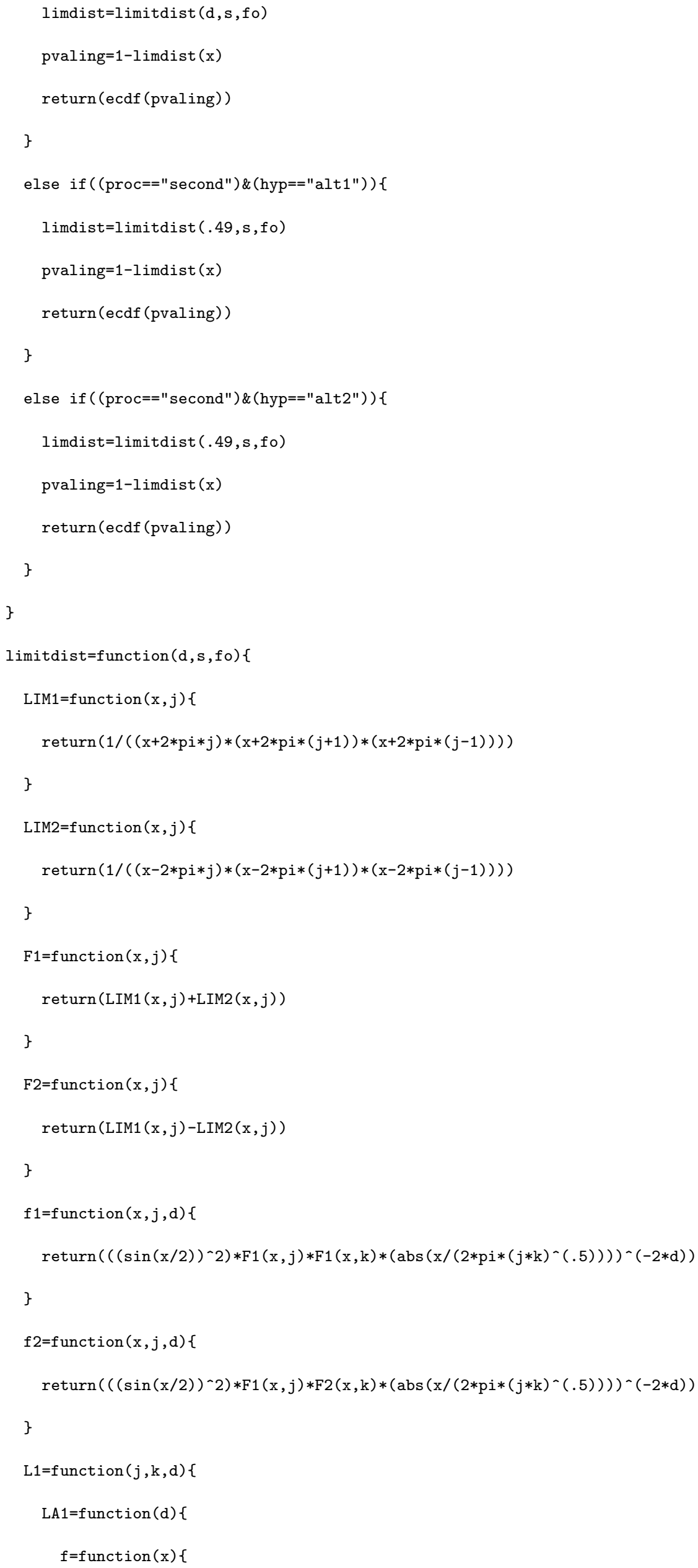


\}

$\operatorname{return}(\operatorname{sapply}(\mathrm{d}, \mathrm{LA} 1))$

\}

$L 2=$ function $(j, k, d)\{$

LA2=function $(d)\{$

$f=$ function $(x)\{$

$\left(16 * \mathrm{pi}^{\wedge}\{3\} / 3\right) *\left(\left((\sin (\mathrm{x} / 2))^{\wedge} 2\right) * \mathrm{~F} 2(\mathrm{x}, \mathrm{j})\right) * \mathrm{~F} 2(\mathrm{x}, \mathrm{k}) *\left(\operatorname{abs}\left(\mathrm{x} /\left(2 * \mathrm{pi} *(\mathrm{j} * \mathrm{k})^{\wedge}(.5)\right)\right)\right)^{\wedge}(-2 * \mathrm{~d})$

\}

return (integrate (f, - Inf , Inf , stop. on. error=FALSE) \$value)

\}

return (sapply (d,LA2))

\}

$L=$ function $(j, k, d)\{$

$\operatorname{return}(L 1(j, k, d)+L 2(j, k, d))$

\}

L1alt=function $(j, k, d)\{$

$p=j+1$

$q=k+1$

$\operatorname{return}(L 1(p, q, d))$

\}

L2alt $=$ function $(j, k, d)\{$

$p=j+1$

$q=k+1$

$\operatorname{return}(L 2(p, q, d))$

\}

Lalt $=$ function $(j, k, d)\{$

$p=j+1$

$q=k+1$

$\operatorname{return}(L(p, q, d))$

\}

$V 1=$ function $(j, k, d)\{$

$p=j+1$

$q=k+1$

$\operatorname{return}(\mathrm{L} 1(\mathrm{p}, \mathrm{q}, \mathrm{d}))$

\}

$V 2=f$ unction $(j, k, d)\{$ 


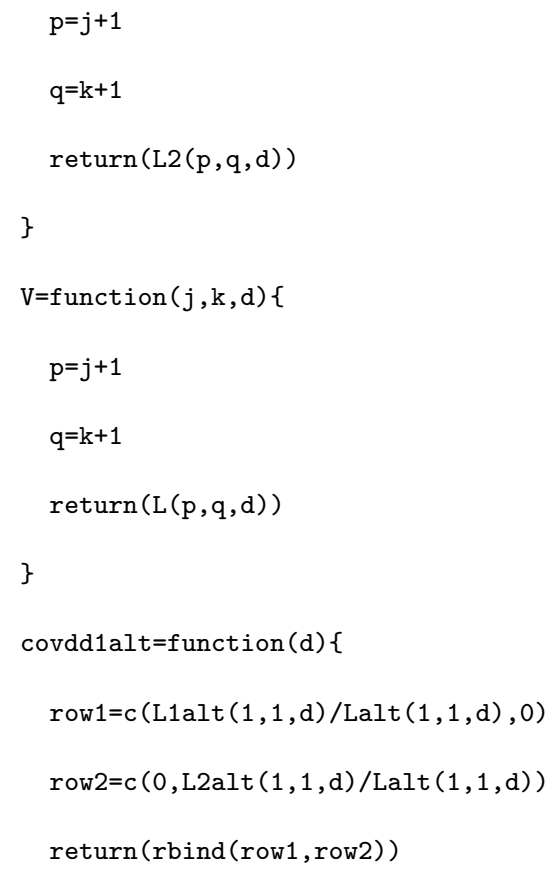


row8 $=c(0,0,0,0, \operatorname{L} 2 a l t(4,1, d) / \operatorname{Lalt}(4,4, d), \operatorname{L2alt}(4,2, d) / \operatorname{Lalt}(4,4, d), \operatorname{L} 2 a l t(4,3, d) / \operatorname{Lalt}(4,4, d), \operatorname{L2alt}(4,4, d) / \operatorname{Lalt}(4,4, d))$ return (rbind (row1, row2, row3, row4, row5, row6, row7, row8))

\}

covdd5alt=function $(d)\{$

row1=c $(V 1(1,1, d) / V(1,1, d), V 1(1,2, d) / V(1,1, d), V 1(1,3, d) / V(1,1, d), V 1(1,4, d) / V(1,1, d), V 1(1,5, d) / V(1,1, d), 0,0,0,0,0)$ row2 $=c(V 1(2,1, d) / V(2,2, d), V 1(2,2, d) / V(2,2, d), V 1(2,3, d) / V(2,2, d), V 1(2,4, d) / V(2,2, d), V 1(2,5, d) / V(2,2, d), 0,0,0,0,0)$ row3 $=c(V 1(3,1, d) / V(3,3, d), V 1(3,2, d) / V(3,3, d), V 1(3,3, d) / V(3,3, d), V 1(3,4, d) / V(3,3, d), V 1(3,5, d) / V(3,3, d), 0,0,0,0,0)$ row $4=c(V 1(4,1, d) / V(4,4, d), V 1(4,2, d) / V(4,4, d), V 1(4,3, d) / V(4,4, d), V 1(4,4, d) / V(4,4, d), V 1(4,5, d) / V(4,4, d), 0,0,0,0,0)$ row $5=c(V 1(5,1, d) / V(5,5, d), V 1(5,2, d) / V(5,5, d), V 1(5,3, d) / V(5,5, d), V 1(5,4, d) / V(5,5, d), V 1(5,5, d) / V(5,5, d), 0,0,0,0,0)$ row6 $=c(0,0,0,0,0, V 2(1,1, d) / V(1,1, d), V 2(1,2, d) / V(1,1, d), V 2(1,3, d) / V(1,1, d), V 2(1,4, d) / V(1,1, d), V 2(1,5, d) / V(1,1, d))$ row $7=c(0,0,0,0,0, V 2(2,1, d) / V(2,2, d), V 2(2,2, d) / V(2,2, d), V 2(2,3, d) / V(2,2, d), V 2(2,4, d) / V(2,2, d), V 2(2,5, d) / V(2,2, d))$ row8 $=c(0,0,0,0,0, V 2(3,1, d) / V(3,3, d), V 2(3,2, d) / V(3,3, d), V 2(3,3, d) / V(3,3, d), V 2(3,4, d) / V(3,3, d), V 2(3,5, d) / V(3,3, d))$ row9 $=c(0,0,0,0,0, V 2(4,1, d) / V(4,4, d), V 2(4,2, d) / V(4,4, d), V 2(4,3, d) / V(4,4, d), V 2(4,4, d) / V(4,4, d), V 2(4,5, d) / V(4,4, d))$ row $10=c(0,0,0,0,0, V 2(5,1, d) / V(5,5, d), V 2(5,2, d) / V(5,5, d), V 2(5,3, d) / V(5,5, d), V 2(5,4, d) / V(5,5, d), V 2(5,5, d) / V(5,5, d))$ return (rbind (row1, row2, row3, row4, row5, row6, row7, row8, row9, row10))

\} covdd1 $=$ function $(d)\{$

\}

$\operatorname{covdd} 2=$ function $(d)\{$

$\operatorname{row} 1=c(L 1(1,1, d) / L(1,1, d), L 1(1,2, d) / L(1,1, d), 0,0)$

row $2=c(L 1(2,1, d) / L(2,2, d), L 1(2,2, d) / L(2,2, d), 0,0)$

$\operatorname{row} 3=c(0,0, L 2(1,1, d) / L(1,1, d), L 2(1,2, d) / L(1,1, d))$

$\operatorname{row} 4=c(0,0, L 2(2,1, d) / L(2,2, d), L 2(2,2, d) / L(2,2, d))$

$\operatorname{return}(r b i n d(r o w 1$, row2, row3, row4))

\}

covdd2alt=function $(d)\{$

$\operatorname{row} 1=c(L 1(2,2, d) / L(2,2, d), L 1(2,3, d) / L(2,2, d), 0,0)$

row2 $=c(L 1(3,2, d) / L(3,3, d), L 1(3,3, d) / L(3,3, d), 0,0)$

row3 $=c(0,0, L 2(2,2, d) / L(2,2, d), L 2(2,3, d) / L(2,2, d))$

row $4=c(0,0, L 2(3,2, d) / L(3,3, d), L 2(3,3, d) / L(3,3, d))$

return (rbind (row1, row2, row3, row4))

\}

$\operatorname{covdd} 3=$ function $(d)\{$

$\operatorname{row} 1=c(L 1(1,1, d) / L(1,1, d), L 1(1,2, d) / L(1,1, d), L 1(1,3, d) / L(1,1, d), 0,0,0)$

row2 $=c(L 1(2,1, d) / L(2,2, d), L 1(2,2, d) / L(2,2, d), L 1(2,3, d) / L(2,2, d), 0,0,0)$ 


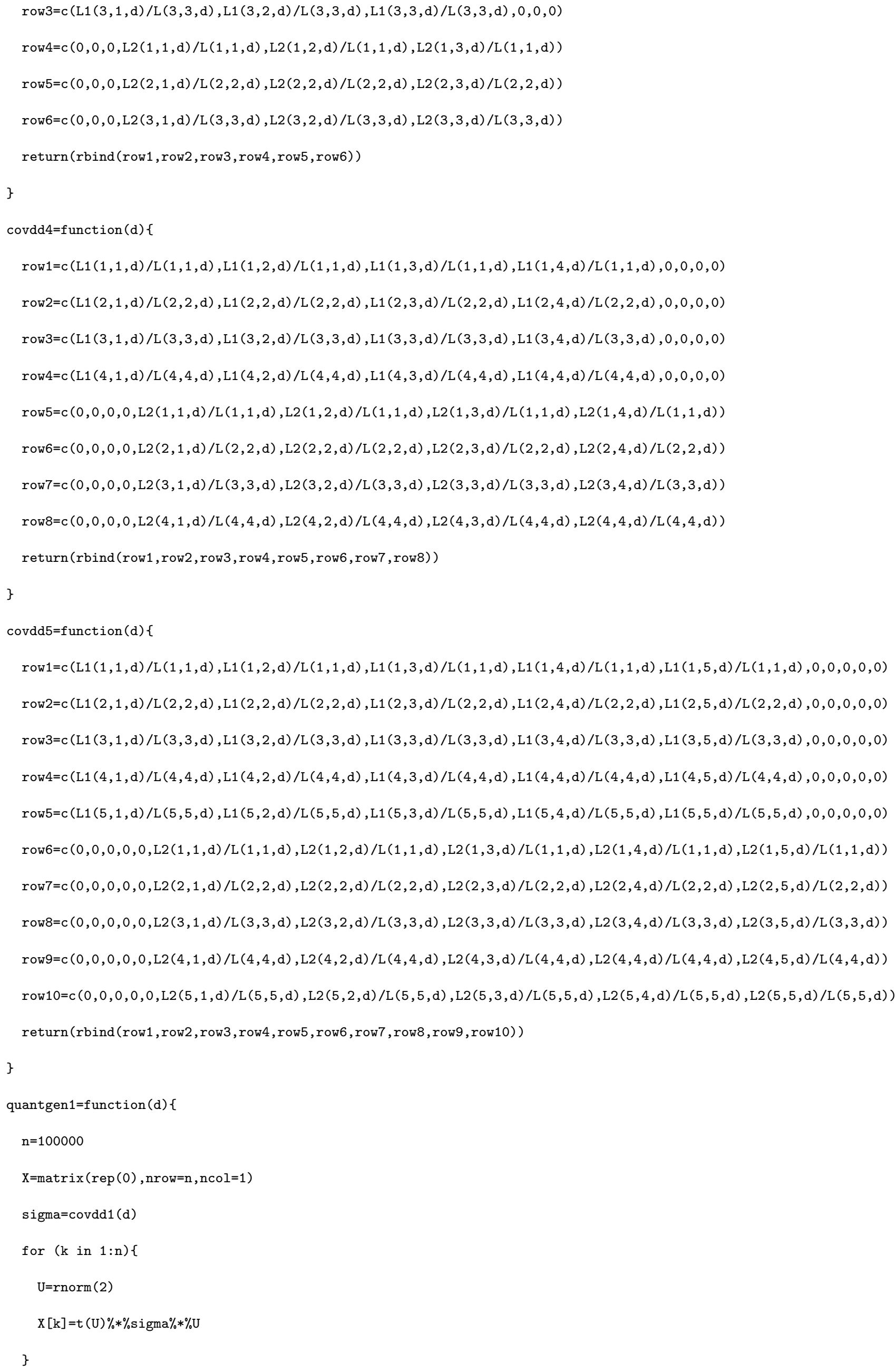


$\operatorname{return}(\mathrm{X})$

\}

quantgen $2=$ function $(d)\{$

$\mathrm{n}=100000$

$\mathrm{X}=\operatorname{matrix}(\operatorname{rep}(0), \mathrm{nrow}=\mathrm{n}, \mathrm{ncol}=1)$

$\operatorname{sigma}=\operatorname{covdd} 2(d)$

for $(k$ in $1: n)\{$

$\mathrm{U}=\operatorname{rnorm}(4)$

$\mathrm{X}[\mathrm{k}]=\mathrm{t}(\mathrm{U}) \% * \%$ sigma $\% * \% \mathrm{U}$

\}

$\operatorname{return}(\mathrm{X})$

\}

quantgen $3=$ function $(d)\{$

$n=100000$

$\mathrm{X}=\operatorname{matrix}(\operatorname{rep}(0), \mathrm{nrow}=\mathrm{n}, \mathrm{ncol}=1)$

$\operatorname{sigma}=\operatorname{covdd} 3(d)$

for $(\mathrm{k}$ in $1: \mathrm{n})\{$

$\mathrm{U}=\mathrm{rnorm}(6)$

$\mathrm{X}[\mathrm{k}]=\mathrm{t}(\mathrm{U}) \% * \%$ sigma $\% * \% \mathrm{U}$

\}

$\operatorname{return}(\mathrm{X})$

\}

quantgen $4=$ function (d) \{

$\mathrm{n}=100000$

$\mathrm{X}=\operatorname{matrix}(\operatorname{rep}(0)$, nrow $=\mathrm{n}, \mathrm{ncol}=1)$

$\operatorname{sigma}=\operatorname{covdd} 4(d)$

for $(k$ in $1: n)\{$

$\mathrm{U}=\mathrm{rnorm}(8)$

$\mathrm{X}[\mathrm{k}]=\mathrm{t}(\mathrm{U}) \% * \%$ sigma $\% * \% \mathrm{U}$

\}

return (X)

quantgen $5=$ function $(d)\{$

$n=100000$

$\mathrm{X}=\operatorname{mat} \operatorname{rix}(\operatorname{rep}(0), \mathrm{nrow}=\mathrm{n}, \mathrm{ncol}=1)$

$\operatorname{sigma}=\operatorname{covdd} 5(d)$

for $(k$ in $1: n)\{$

$\mathrm{U}=\mathrm{rnorm}(10)$ 
$X[k]=t(U) \% * \%$ sigma $\% * \%$

\}

$\operatorname{return}(\mathrm{X})$

\}

quantgen1alt=function $(d)\{$

$n=100000$

$\mathrm{X}=\operatorname{matrix}(\operatorname{rep}(0)$, nrow=n, ncol=1)

sigma $=$ covdd 1 alt $(d)$

for $(k$ in $1: n)\{$

$\mathrm{U}=\mathrm{rnorm}(2)$

$\mathrm{X}[\mathrm{k}]=\mathrm{t}(\mathrm{U}) \% * \%$ sigma $\% * \% \mathrm{U}$

\}

$\operatorname{return}(\mathrm{X})$

\}

quantgen2alt $=$ function $(d)\{$

$n=100000$

$\mathrm{X}=\operatorname{matrix}(\operatorname{rep}(0)$, nrow=n, ncol=1)

sigma $=\operatorname{covdd} 2 a l t(d)$

for $(\mathrm{k}$ in $1: \mathrm{n})\{$

$\mathrm{U}=\operatorname{rnorm}(4)$

$\mathrm{X}[\mathrm{k}]=\mathrm{t}(\mathrm{U}) \% * \%$ sigma $\% * \% \mathrm{U}$

\}

$\operatorname{return}(\mathrm{X})$

\}

quantgen3alt=function $(d)\{$

$\mathrm{n}=100000$

$\mathrm{X}=\operatorname{matrix}(\operatorname{rep}(0)$, nrow $=\mathrm{n}, \mathrm{ncol}=1)$

sigma $=$ covdd $3 a l t ~(d)$

for $(k$ in $1: n)\{$

$\mathrm{U}=\mathrm{rnorm}(6)$

$\mathrm{X}[\mathrm{k}]=\mathrm{t}(\mathrm{U}) \% * \%$ sigma $\% * \% \mathrm{U}$

\}

$\operatorname{return}(\mathrm{X})$

\}

quantgen4alt $=$ function $(d)\{$

$\mathrm{n}=100000$

$\mathrm{X}=\operatorname{matrix}(\operatorname{rep}(0)$, nrow $=\mathrm{n}, \mathrm{ncol}=1)$

sigma $=\operatorname{covdd} 4$ alt $(d)$ 
for $(\mathrm{k}$ in $1: \mathrm{n})\{$

$\mathrm{U}=\operatorname{rnorm}(8)$

$\mathrm{X}[\mathrm{k}]=\mathrm{t}(\mathrm{U}) \% * \%$ sigma $\% * \% \mathrm{U}$

\}

$\operatorname{return}(\mathrm{X})$

\}

quantgen5alt=function (d) \{

$\mathrm{n}=100000$

$\mathrm{X}=\operatorname{matrix}(\operatorname{rep}(0), \mathrm{nrow}=\mathrm{n}, \mathrm{ncol}=1)$

sigma $=\operatorname{covdd} 5 a l t(d)$

for $(k$ in $1: n)\{$

$\mathrm{U}=\mathrm{rnorm}(10)$

$\mathrm{X}[\mathrm{k}]=\mathrm{t}(\mathrm{U}) \% * \%$ sigma $\% * \% \mathrm{U}$

\}

$\operatorname{return}(\mathrm{X})$

\}

if $((s==1) \&(f o==" n o "))\{$

$\operatorname{return}(\operatorname{ecdf}($ quantgen1 (d)))

else if $((s==2) \&(f o==" n o "))\{$

return (ecdf (quantgen2(d)))

\}

else if $((s==3) \&(f o==" n o "))\{$

$\operatorname{return}(\operatorname{ecdf}($ quantgen3(d)))

\}

else if $((s==4) \&(f o==" n o "))\{$

return (ecdf (quantgen4(d)))

\}

else if $((s==5) \&(f o==" n o "))\{$

$\operatorname{return}($ ecdf (quantgen5(d)))

\}

if $((s==1) \&(f o=="$ yes" $))\{$

return (ecdf (quantgen1alt (d)))

\}

else if $((s==2) \&($ fo=="yes" $))\{$

return (ecdf (quantgen2alt (d)))

else if $((s==3) \&($ fo= $=$ "yes" $))\{$ 


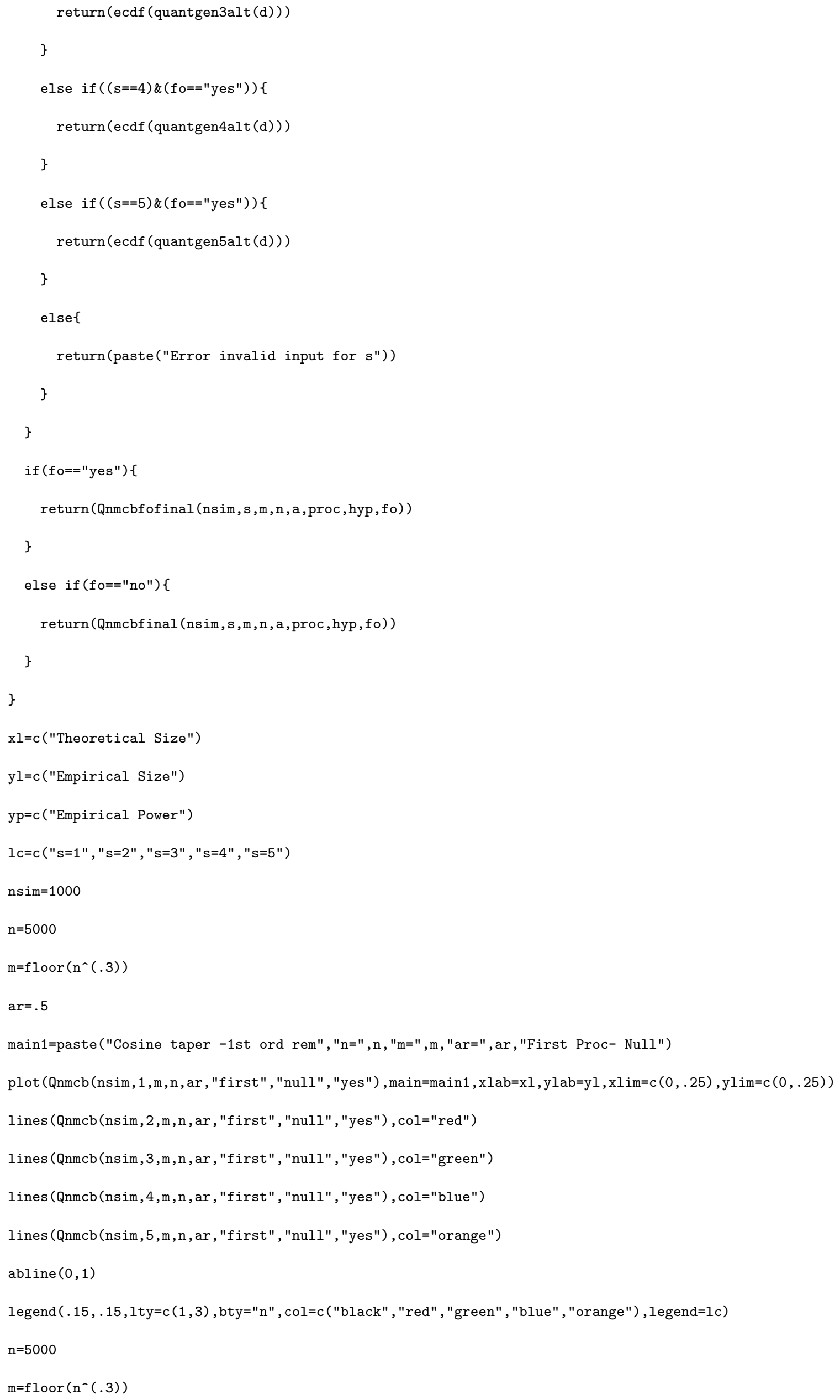


main1=paste ("Cosine -1st ord", "n=",n, "m=",m, "d=", d, "First Proc- alt")

plot (Qnmcb(nsim,1,m,n,d, "first", "alt", "yes"), main=main1, xlab=xl, ylab=yp, xlim=c $(0,1), y l i m=c(0,1))$

lines (Qnmcb(nsim, 2,m,n,d, "first" , "alt", "yes"), col="red")

lines (Qnmcb(nsim, 3,m,n,d, "first", "alt", "yes"), col="green")

lines (Qnmcb(nsim, 4,m,n, d, "first", "alt", "yes"), col="blue")

lines (Qnmcb (nsim, 5,m,n,d, "first" , "alt", "yes"), col="orange")

abline $(0,1)$

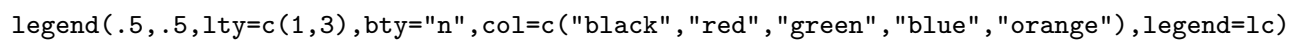

$n=5000$

$m=f \operatorname{loor}\left(n^{-}(.3)\right)$

$\mathrm{d}=.4$

main1=paste ("Cosine -1st ord rem", "n=",n, "m=",m, "d=", d, "Second Proc- Null")

plot (Qnmcb(nsim, 1,m,n, d, "second" , "null" , "yes"), main=main1, xlab=xl,ylab=yl, xlim=c (0, .25),ylim=c (0, .25))

lines (Qnmcb (nsim, 2, m, n, d, "second", "null", "yes"), col="red")

lines (Qnmcb (nsim, 3,m,n, d, "second" , "null", "yes"), col="green")

lines (Qnmcb (nsim, 4,m,n, d, "second" , "null" , "yes") , col="blue")

lines (Qnmcb (nsim, 5,m,n, d, "second", "null", "yes"), col="orange")

abline $(0,1)$

legend $(.5, .5,1$ ty=c $(1,3), b t y=" n ", c o l=c(" b l a c k "$, "red", "green", "blue" , "orange"), legend=lc)

$\mathrm{n}=5000$

$m=f \operatorname{loor}\left(n^{-}(.3)\right)$

$\operatorname{ar}=.5$

main1=paste ("Cosine -1st ord rem", "n=",n, "m=",m, "ar=", ar, "Second Proc- Alt - Trend")

plot (Qnmcb(nsim, 1,m,n, ar, "second" , "alt1", "yes"), main=main1, xlab=xl, ylab=yp, xlim=c $(0,1), y l i m=c(0,1))$

lines (Qnmcb (nsim, 2,m,n, ar, "second", "alt1", "yes") , col="red")

lines (Qnmcb (nsim, 3,m,n, ar, "second", "alt1", "yes"), col="green")

lines (Qnmcb (nsim, 4,m, n, ar, "second", "alt1", "yes"), col="blue")

lines (Qnmcb (nsim, 5,m,n, ar, "second", "alt1", "yes"), col="orange")

abline $(0,1)$

legend $(.5, .5,1$ ty=c $(1,3)$, bty="n", col=c ("black", "red" , "green", "blue" , "orange"), legend=lc)

$\mathrm{n}=1000$

$m=f \operatorname{loor}\left(n^{-}(.3)\right)$

$\operatorname{ar}=.5$

main1=paste ("Cosine -1st ord rem", "n=",n, "m=",m, "ar=", ar, "Second Proc- Alt - Rndm Wlk")

plot (Qnmcb(nsim, 1,m,n, ar, "second", "alt2", "yes"), main=main1, xlab=xl, ylab=yp, xlim=c $(0,1), y l i m=c(0,1))$

lines (Qnmcb (nsim, 2,m,n, ar, "second" , "alt2", "yes") , col="red")

lines (Qnmcb (nsim, 3,m,n, ar, "second", "alt2", "yes"), col="green")

lines (Qnmcb (nsim, 4,m,n, ar, "second", "alt2" , "yes"), col="blue") 
lines (Qnmcb (nsim, 5,m,n, ar, "second", "alt2", "yes"), col="orange")

abline $(0,1)$

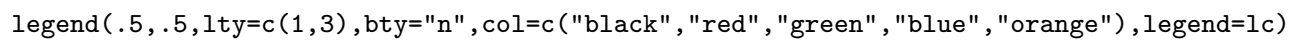

$\mathrm{n}=5000$

$m=f \operatorname{loor}\left(n^{-}(.3)\right)$

$\operatorname{ar}=.5$

main1=paste ("Cosine Taper", "n=",n, "m=",m, "ar=" , ar, "First Proc- Null")

plot (Qnmcb(nsim,1,m,n,ar, "first", "null" , "no"), main=main1, xlab=xl, ylab=yl, xlim=c (0, . 25),ylim=c (0, .25))

lines (Qnmcb(nsim, 2,m,n,ar, "first" , "null" , "no"), col="red")

lines (Qnmcb (nsim, 3,m,n, ar, "first", "null", "no"), col="green")

lines (Qnmcb (nsim, 4,m,n, ar, "first", "null", "no") , col="blue" )

lines (Qnmcb (nsim, 5,m,n, ar, "first" , "null", "no"), col="orange")

abline $(0,1)$

legend $(.5, .5,1$ ty=c $(1,3)$, bty="n", col=c ("black", "red", "green", "blue" , "orange"), legend=lc)

$\mathrm{n}=1000$

$m=f \operatorname{loor}\left(n^{\wedge}(.3)\right)$

$d=.4$

main1=paste ("Cosine Taper", "n=",n, "m=",m, "d=", d, "First Proc- alt")

plot (Qnmcb(nsim,1,m,n,d, "first" , "alt" , "no"), main=main1, xlab=xl, ylab=yp, xlim=c $(0,1), y l i m=c(0,1)$ )

lines (Qnmcb (nsim, 2,m,n,d, "first", "alt", "no"), col="red")

lines (Qnmcb (nsim, 3,m,n, d, "first", "alt", "no"), col="green")

lines (Qnmcb (nsim, 4,m,n, d, "first", "alt", "no"), col="blue")

lines (Qnmcb (nsim, 5,m,n, d, "first", "alt", "no"), col="orange")

abline $(0,1)$

legend $(.5, .5,1$ ty=c $(1,3)$, bty="n", col=c ("black", "red", "green", "blue" , "orange"), legend=lc)

$\mathrm{n}=5000$

$m=f \operatorname{loor}\left(n^{-1}(.3)\right)$

$d=.4$

main1=paste ("Cosine Taper", "n=",n, "m=",m, "d=",d, "Second Proc- Null")

plot (Qnmcb(nsim, 1,m,n,d, "second" , "null" , "no"), main=main1, xlab=xl, ylab=yl, xlim=c (0, .25),ylim=c (0, .25))

lines (Qnmcb (nsim, 2, m, n, d, "second" , "null", "no"), col="red")

lines (Qnmcb (nsim, 3,m,n,d, "second", "null", "no"), col="green")

lines (Qnmcb (nsim, 4,m,n, d, "second", "null", "no"), col="blue")

lines (Qnmcb (nsim, 5,m,n, d, "second" , "null", "no"), col="orange")

abline $(0,1)$

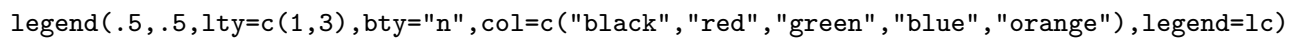

$\mathrm{n}=5000$

$m=f \operatorname{loor}\left(n^{-}(.3)\right)$ 
$\operatorname{ar}=.5$

main1=paste ("Cosine Taper", "n=",n, "m=",m, "ar=", ar, "Second Proc- Alt - Trend")

plot (Qnmcb(nsim,1,m,n,ar, "second" , "alt1" , "no"), main=main1, xlab=xl,ylab=yp, xlim=c $(0,1), y l i m=c(0,1))$

lines (Qnmcb (nsim, 2,m,n, ar, "second", "alt1", "no") , col="red")

lines (Qnmcb (nsim, 3,m,n, ar, "second" , "alt1", "no") , col="green")

lines (Qnmcb (nsim, 4,m,n, ar, "second", "alt1", "no") , col="blue")

lines (Qnmcb (nsim, 5,m,n, ar, "second", "alt1", "no"), col="orange")

abline $(0,1)$

legend $(.5, .5,1$ ty=c $(1,3)$, bty="n", col=c ("black", "red" , "green" , "blue" , "orange"), legend=lc)

$\mathrm{n}=5000$

$m=f \operatorname{loor}\left(n^{\wedge}(.3)\right)$

$\operatorname{ar}=.5$

main1=paste ("cosine", "n=",n, "m=",m, "ar=", ar, "second Proc- Alt - Random Walk")

plot (Qnmcb (nsim, 1,m,n, ar, "second" , "alt2", "no"), main=main1, xlab=xl, ylab=yp, xlim=c $(0,1), y l i m=c(0,1)$ )

lines (Qnmcb (nsim, 2,m,n, ar, "second", "alt2", "no") , col="red")

lines (Qnmcb (nsim, 3,m,n, ar, "second", "alt2", "no") , col="green")

lines (Qnmcb (nsim, 4,m,n, ar, "second", "alt2", "no") , col="blue")

lines (Qnmcb (nsim, 5,m, n, ar, "second", "alt2", "no"), col="orange")

abline $(0,1)$

legend $(.5, .5,1$ ty=c $(1,3)$, bty="n", col=c ("black", "red", "green", "blue" , "orange"), legend=lc) 


\section{References}

[1] J. Beran, R. Kulik, S. Ghosh, and Y. Feng (2013). Long-Memory Processes: Probabilistic Properties and Statistical Methods. Springer, 1st Edition.

[2] R. Berger, and G. Casella (2002). Staistical Inference. Thomson Learning, 2nd Edition.

[3] P. Billingsley (1995). Probability and Measure. John Wiley and Sons, 3rd Edition.

[4] G. Box, G. Jenkins, and G. Reinsel (2008). Wiley, 4th Edition.

[5] M. Busch and P. Sibbertsen (2018). An Overview of Modified Semiparamteric Memory Estimation. Hannover Economic Papers, dp-628.

[6] M. Csorgo, M. Nasari, and M. Ould Haye (2017). Bootstrapped Pivots for Means of Short and Long Memory Linear Processes. Bernoulli, 23.4A.

[7] R. Davidson and J. MacKinnon (1997). Graphical Methods for Investigating the Size and Power of Hypothesis Tests. Working Paper.

[8] R. Deo (1997). Asymptotic Theory For Certain Regression Models with Long Memory Errors. Journal of Time Series Analysis, 18.4.

[9] J. Geweke and S. Porter Hudak (1983). The Estimation and Application of Long Memory Time Series Models. Journal of Time Series Analysis, 40.4. 
[10] L. Giraitis, R. Leipus, and A. Philippe (2006). A Test for Stationarity versus Trends and Unit Roots for a Wide Class of Dependent Errors. Econometric Theory, 22.6.

[11] L. Giraitis, R. Leipus, P. Kokoszka, and G. Teyssiere (2003). Rescaled Variance and Related Tests for Long Memory in Volatility and Levels. Econometrics, 112.2.

[12] L. Giraitis, H. Koul, and D. Surgailis (2012). Large Sample Inference for Long Memory Processes. Imperial College Press.

[13] C. Gotway and O. Schabenberger (2004). Statistical Methods for Data Analysis. Chapman and Hall/CRC Press, 1st Edition.

[14] G. Gromykov, M. Ould Haye, and A. Phillippe (2018). A Frequency-Domain Test for Long Range Depdendence. Statistical Inference for Stochastic Proccesses, 21.3.

[15] H. Hurst (1951). Long-term Storage Capacity of Reservoirs. Transactions of the American Society of Civil Engineers, 116.

[16] C. Hurvich and K. Beltrao (1993). Aymptotics for the Low-Frequency Ordinates of the Periodogram of a Long-Memory Time Series. Journal of Time Series Analysis, 14.5. 
[17] C. Hurvich and B. Ray (1995). Estimation of the Memory Parameter for NonStationary or Non-Invertible Fractionally Integrated Processes. Journal of Time Series Analysis, 16.1.

[18] D. Lee and P. Schmidt (1996). On the Power of the KPSS Test of Stationarity Against Fractionally Integrated Alternatives. Journal of Econometrics, 73.1.

[19] A. Lo (1991). Long-Term Memory in Stock Market Prices. Econometrica, 58.5.

[20] I. Lobato (1997). Consistency of the Averaged Cross Periodogram in Long Memory Series. Journal of Time Series Analysis, 18.2.

[21] I. Lobato and P. Robinson (1998). A Nonparametric Test for I(0). Review of Economic Studies, 65.3.

[22] M. Ould Haye and A. Phillippe (2019). Frequency Test for Stationarity in Dependent Data. Working Paper.

[23] M. Priestley. (1981). Spectral Analysis and Time Series Volume I. Academic Press, 1st Edition.

[24] P. Robinson. (1995). Gaussian Semiparametric Estimation of Long Range Dependence. The Annals of Statistics, 23.5.

[25] R. Shumway and D. Stoffer (2011). Time Series Analysis and Its Applications: With R Examples. Springer, 4th Edition. 
[26] M. Taqqu, V. Teverovsky, and W. Willinger (1999a). Stock Market Prices and Long-Range Dependence. Finance and Stochastics, 3.1.

[27] M. Taqqu, V. Teverovsky, and W. Willinger (1999b). On Lo's Modified R/S Statistic. Journal of Statistical Planning and Inference, 80.1.

[28] C. Velasco (1999a). Non-Stationary Log-Periodogram Regression. Journal of Econometrics 91.

[29] C. Velasco. (1999b) Gaussian Semiparametric Estimation of Non-Stationary Time Series. Journal of Time Series Analysis, 20.1.

[30] W. Wei (1991). Time Series Analysis: Univariate and Multivariate Methods. Addison-Wesley, 1st Edition. 